\title{
The Role of Abiotic Soil Parameters as a Factor in the Success of Invasive Plant Species
}

\author{
G.W.W. Wamelink ${ }^{a^{*}}$, H.F. van Dobben ${ }^{\text {a }}$, P.W. Goedhart ${ }^{\text {b }}$, L.M. Jones-Walters ${ }^{\text {a }}$ \\ ${ }^{a}$ Wageningen Environmental Research, Wageningen UR, P.O. box 47, Wageningen, the Netherlands \\ ${ }^{b}$ Biometris, Wageningen UR, P.O. Box 16, 6700 AA Wageningen, The Netherlands
}

\begin{abstract}
Plant species dispersal has been strongly enhanced by human activities. Introduced species have to cope with indigenous species and local conditions. They may avoid indigenous species by occupying new (abiotic) territory. Once a species is established it may become a pest, and may seriously threaten other species and ecosystems.

In this paper we focus on invasive plant species of the Dutch flora. We make two comparisons: (1) Dutch neophytes (i.e. arrived in The Netherlands after 1825) vs. indigenous Dutch flora; and (2) species of the Dutch flora that have become invasive outside Europe vs. non-invasive species of the Dutch flora. We hypothesize that at least part of the success of the invasive or neophyte species is due to their ability to grow under a wider range of abiotic soil circumstances than other species. We regard an invasive species as successful if it is able to disperse from the introduction site(s) and remain presen in the invaded vegetation.

For ten out of the sixteen abiotic factors there is a wider range for the neophytes: chlorine, potassium, mean highest and lowest groundwater level, phosphorus (and total content) and pH H2O.

We hypothesized that part of the success of invasive species may be the adaptation to a variety of abiotic soil parameters. This is indeed the case for a number of the examined parameters, mostly related to nutrient availability and soil $\mathrm{pH}$. This indicates that the success of invasive species is at least partly caused by their ability to grow under a wide range of nutrient availability and soil $\mathrm{pH}$. Their success may therefore be stimulated by the increasing pollution of natural areas by excessive nitrogen.
\end{abstract}

\section{Keywords:}

Soil pH;

Exotic Species;

Alien Species;

Neophytes;

Abiotic Conditions;

Species Responses.

\section{Article History:}

Received: 27 October 2018

Accepted: 10 December 2018

\section{1- Introduction}

It is a characteristic of species that they disperse, often across geographical areas, in order to find suitable new habitats [1, 2]. During past millennia, species dispersal has been strongly enhanced by human activities. Such activities include, for example, the active displacement of agricultural or ornamental species and the fungi, plants and animals that may be associated with them [3], or the creation of new dispersal pathways such as the Rhine-Danube canal [4].

When new species arrive in existing ecosystems they have to compete with the species present, especially when they have a similar preferences. If the newly arrived species is unable to compete with the species that are already there it will perish. Alternatively, the new species may occupy new (abiotic) territory. Once a species is established it may become a pest, sometimes after multiplying exponentially, and may seriously threaten other species and ecosystems [5, 6]. As a result, species may even be threatened by extinction; there are numerous examples where invasive species have led to local and regional extinctions. Some of the best known examples come from islands in the Pacific Ocean where, especially between the 17th and 19th centuries, sailors were in the habit of leaving domesticated animals as emergency food in case of a future shipwreck or emergency need. Among them were goats and pigs which had a devastating effect

*CONTACT: Wieger.wamelink@wur.nl

DOI: http://dx.doi.org/10.28991/esj-2018-01155

(C) This is an open access article under the CC-BY license (https://creativecommons.org/licenses/by/4.0/). 
on the local ecosystems [7, 8]. The introduction of the plant Alliaria petiolata (Bieb.) Cavara \& Grande (garlic mustard) to North America in the late 19th century led to changes in plant communities, and even to fungal communities by disrupting mutualistic associations between fungal and higher plant species [9].

Vanderhoeven et al. [10] showed that several invasive species were able to change abiotic soil conditions. On the other hand, some invasive species which were at first considered pests have, after some time, become integrated into the local ecosystems $[11,12]$.

How should we assess the value or otherwise of these newcomers? The rabbit (Oryctolagus cuniculus L.) was possibly introduced to the Netherlands by the Romans, but most likely imported from France at least 750 years ago. It is now a highly valued member of Dutch nature as an 'ecosystem engineer' [13]. It should be noted, however, that it is still considered a severe pest in many countries around the world [14]. Rosa rugosa Thunb. (Beach rose) is an invasive plant species that threatens existing ecosystems in North-West Europe and which has been shown to reduce species diversity; specifically on the German Island of Spiekeroog at sites it invaded [15]. Another invasive plant species in Europe [16] with the disputed potential to affect the local flora, Impatiens glandulifera Royle (Himalayan balsam), is also valued for its nectar production [11, 12]. Invasive species may therefore be a threat or, in some cases over time, may become a neutral or even occasionally valuable addition to ecosystems. The role of a newcomer is not known beforehand and, given the potential for newly-arrived species to damage existing ecosystems and the services they provide and in some cases to inflict severe economic impacts it would be useful to have a tool to estimate the potential of a species to become invasive.

The European Union (EU) has recognized the threat of invasive species. A new EU policy was adopted on 1 January 2015 which 'seeks to address the problem of invasive alien species in a comprehensive manner so as to protect indigenous biodiversity and ecosystem services, as well as to minimize and mitigate the human health or economic impacts that these species can have [17]. Examples of invasive species are Fallopia japonica (Houtt.) Ronse Decr. (Japanese knotweed) with major ecological consequences and Ambrosia artemisiifolia L. (common ragweed) which has serious allergenic properties for people and for domestic animals [18-20]. The regulation foresees three types of interventions: prevention, early warning and rapid response and management [21].

Invasive plant species may be able to maintain themselves or become pests for several reasons. The lack of natural enemies such as herbivores or pathogens in their new environment is one of the major reasons [22-24] (Figure 1 in Wardle et al. [25]). Sites that have a relatively low diversity may also be more vulnerable for invasive species [26, 27]. More recently it has become clear that the success of some invasive species may be enhanced by their interactions with fungi [28-31]. Invasive species may even shift their natural preferences [32] or undergo accelerated evolution [33]. Here we study the role of a species' response to abiotic conditions as a predictor of its invasive potential.

Climate change may make some species more prone to migrate than before. This will bring a new class of species with the potential to become invasive, the 'climate refugee'. Unlike the invasive species introduced through human activity, we may welcome these species, since the alternative for them may be extinction or they may fulfil the functional roles of existing species that could become extinct under the influence of changing climate [34]. Nevertheless, these climate refugees may also become pests in their new habitat. Information on the traits of these species may therefore help to assess beforehand if they have the potential to become a risk.

In this paper we focus on 52 invasive plant species of the Dutch flora, of which 37 are listed by IUCN in the top 100 world's worst invasive species [35]. Invasive plant species can have devastating effects on ecosystems; well-known examples are F. japonica, I. glandulifera or Prunus serotina Ehrh. (Black cherry) in Europe and Acer platanoides L. (Norway maple) or Alliaria petiolata (Bieb.) Cavara \& Grande (garlic mustard) in the US. Such species may out-compete other species and form monocultures over large areas, influencing the nutrient cycle, productivity and community structure and function $[36,37]$. When established they may even change local abiotic circumstances and act as ecoengineers [38].

Soil is a major factor that determines the occurrence of plant species [39], besides land use [40], grazing [41, 42] and seed dispersal [43, 44]. Earlier research showed that in general rare species may have narrower preferences for abiotic soil parameters than common species [45]. Since neophytes are successful and can even become pests, we hypothesize that at least part of the success of invasive species is caused by their ability to grow under a wide range of abiotic soil circumstances. To test this hypothesis we compared the abiotic ranges relative to soil parameters for indigenous Dutch species with invasive species in the Netherlands. We also investigated whether there are differences between noninvasive species that are components of the indigenous Dutch flora and Dutch species that became invasive outside Europe.

\section{2- Materials and Methods}

The ranges of the species were estimated using two different databases, the Ecological Conditions Database (ECDatabase [46]) and a sub selection of the National Dutch Vegetation Database (NDV [47]). Both databases have an entry 
in the 'Global index of vegetation-plot databases (GIVD)' under number EU-NL-001 (https://www.givd.info/ID/EUNL-001) and EU-00-006; https://www.givd.info/faces/databases.xhtml). In short, response functions were estimated for plant species based on the EC-database for abiotic soil parameters such as soil $\mathrm{pH}$ or calcium content based on species present in a relevé and measured soil parameter. The optima of the species per abiotic parameter where used to estimate the soil parameters for the relevés in the NDV database (which lack abiotic field measurements). The estimated soil parameters where combined with the species in the relevés and then again the species responses were estimated and the ranges of occurrence of the species, used in this research.

\section{2-1- Databases}

Species responses for abiotic soil parameters (Table 1) were estimated on the basis of field measurements in 8632 plots in the Netherlands mostly collected from literature and for a minor part recorded by the authors (Ecological Conditions (EC) Database). For each plot species composition was recorded and a mixed soil sample was taken from the upper soil layer and analysed. Plot sizes ranged from $1 \mathrm{~m} 2$ for grasslands up to $100 \mathrm{~m} 2$ for forest. Plots were recorded following Braun-Blanquet's method [48]. The data cover the period from 1936 to the present, with the majority of the recordings after 1990. Each plot is accompanied by at least one abiotic soil measurement (e.g. pH or nitrate content). The most frequently measured soil parameter is $\mathrm{pH}$, with 5,914 entries (see further Table 1). The database is part of the European metadata database for vegetation plots and it has previously been described by Wamelink et al. [46].

The National Dutch Vegetation database [47] had at 1-1-2018 over 600.000 entries of vegetation relevés, mostly following the Braun-Blanquet method [48]. The oldest entries date back from the 19th century, with the major part of the entries dating after 1950. The database is updated with new entries but also historical data. The relevés were made by many authors for many different purposes. All these relevés lack soil measurements taken at the site. From the NDV 160.000 relevés were selected which represent the Dutch vegetation and on which the Dutch phytosociological classes are based [49].

\section{2-2- Species Response}

Species responses for soil $\mathrm{pH} \mathrm{H} 2 \mathrm{O}$ were estimated based on combined field measurement of $\mathrm{pH}$ and vegetation plots from the EC-database (see also Wamelink et al. [50]). The measured $\mathrm{pH}$ of the plot was linked to all the species in the plot, including mosses, lichens shrubs and trees.

Responses for $\mathrm{pH}$ per species were modelled by means of logistic regression applying a spline function [51-53] to estimate the curve. Applying the spline function has the advantage that the responses of the species are independent of the number of findings. By dividing the abiotic (x) axes in 25 parts and subsequently calculating the chance of occurrence per part, a bias due to the number of findings is prevented. For each species an indicator value was derived as the optimum of the response curve. This gives responses for only a limited number of species and based on a relative limited number of relevés.

The derived species indicator values were therefore used to calculate the mean abiotic values for a calibration dataset with 160,000 relevés, representative for the Dutch vegetation. For each relevé of the calibration set the soil $\mathrm{pH}$ was calculated as the arithmetic mean of the indicator values for $\mathrm{pH}$ of the species in the relevé. The above described procedure was repeated and new response curves per species were estimated, resulting for most of the Dutch species in an response curve and thus an optimum of the curve. This optimum was used as the new indicator value. The thus derived indicator values per species were used to estimate the soil $\mathrm{pH}$ for the plots used for the estimation of the soil $\mathrm{pH}$ map.

\section{2-3- Comprehensive Description of the Spline Method}

We applied the P-spline technique [51] to estimate the species response curves, analogous to Wamelink et al. [50]. However, some adjustments were made compared to our earlier paper.

The response curve of a species describes the occurrence probability $p$ of the species as a function of an abiotic soil variable x. Gaussian logit curve for species response curves can be defined as:

$$
\log \left(\frac{p}{1-p}\right)=\beta_{0}+\beta_{1} x+\beta_{2} x^{2}
$$

This is, for negative $\beta_{2}$, a symmetric bell-shaped unimodal curve which can, for presence-absence data, conveniently be estimated by means of logistic regression. However, many species response curves are not symmetric. This can sometimes be remedied by increasing the polynomial order of the function, or by transforming the abiotic variable $\mathrm{x}$ such that the curve becomes more symmetric. It can, however, not be remedied when the curve behaves differently in different parts of the range of $\mathrm{x}$. An alternative approach is to divide the range of $\mathrm{x}$ into segments and to fit an appropriate polynomial in each segment while ensuring that adjacent polynomials join smoothly. Splines are precisely used for that 
purpose. Wamelink et al. [52, 53] used a smoothing spline, more generally called generalized additive models [54], to model the relationship with soil $\mathrm{pH}$. They used backward deviance testing for the determination of the degrees of freedom, or flexibility, of the spline. A very similar, but computationally more convenient, smoothing technique is offered by penalized cubic B-splines [51], or P-splines for short, and this was used to estimate the response curves. Some of the background of penalized cubic splines is explained here, largely following Eilers \& Marx [51].

A cubic spline is a piecewise cubic polynomial, with the pieces joined smoothly at certain values of $\mathrm{x}$. These values are called knots. Smoothness can be defined by requiring that the function values and the first two derivatives agree at the knots. A cubic spline with $\mathrm{k}$ knots $t_{1}, t_{2}, \ldots, t_{k}$ which obeys this definition can be written as follows:

$$
S(x)=\beta_{0}+\beta_{1} x+\beta_{2} x^{2}+\beta_{3} x^{3}+\sum_{i=1}^{k} \beta_{i+3}\left(x-t_{i}\right)_{+}^{3}
$$

Where $\left(x-t_{i}\right)_{+}$is zero for values $\mathrm{x}$ smaller that $\mathrm{t} \_\mathrm{i}$, and $\left(x-t_{i}\right)$ otherwise. The set of functions $\left\{x, x^{2}, x^{3},\left(x-t_{1}\right)_{+}^{3}, \ldots,\left(x-t_{k}\right)_{+}^{3}\right\}$ form the so-called truncated polynomial spline basis and the spline $\mathrm{S}(\mathrm{x})$ is a linear combination of these functions. An equivalent but computationally more elegant and stable representation is given by the so-called B-spline basis [55]. An example of a cubic B spline basis is given in Figure 1 in which equidistant knots at integer values are used. Figure 1(a) displays the B spline basis functions B_j (x), while Figures 1(b) and 1(c) display two different linear combinations $S(x)=\sum_{j} \beta_{j} B_{j}(x)$.

(a)

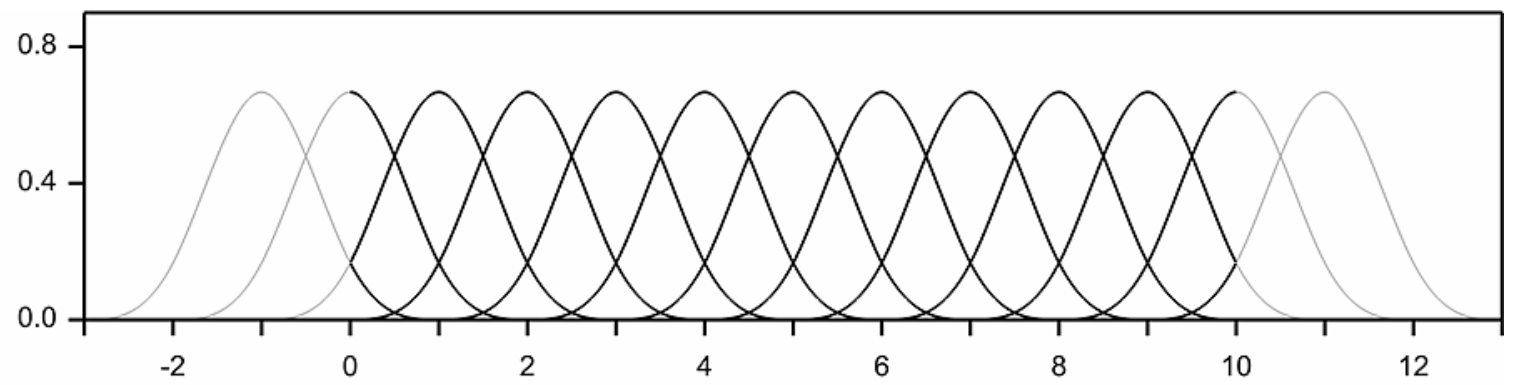

(b)

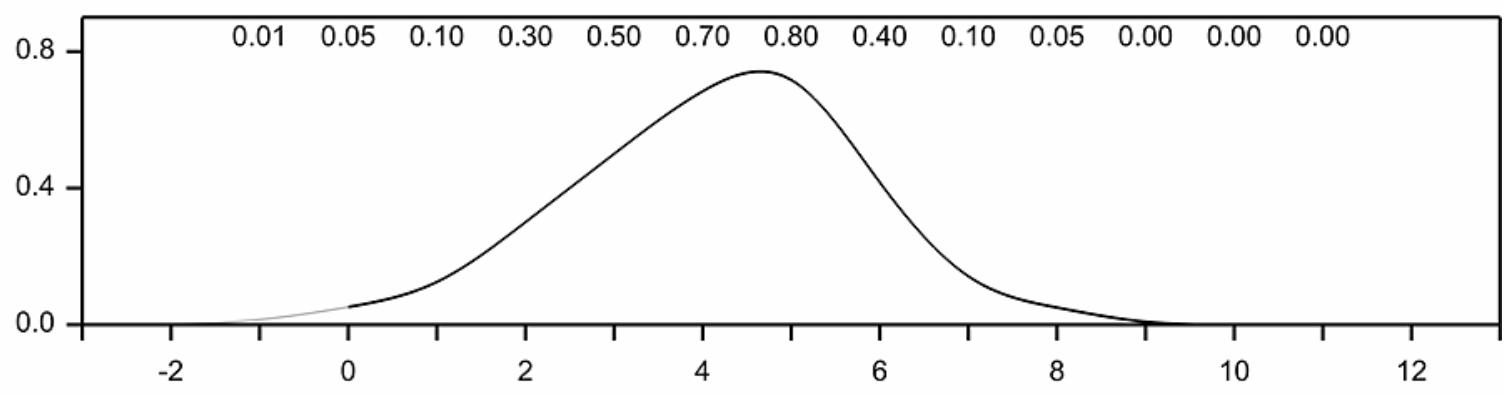

(c)

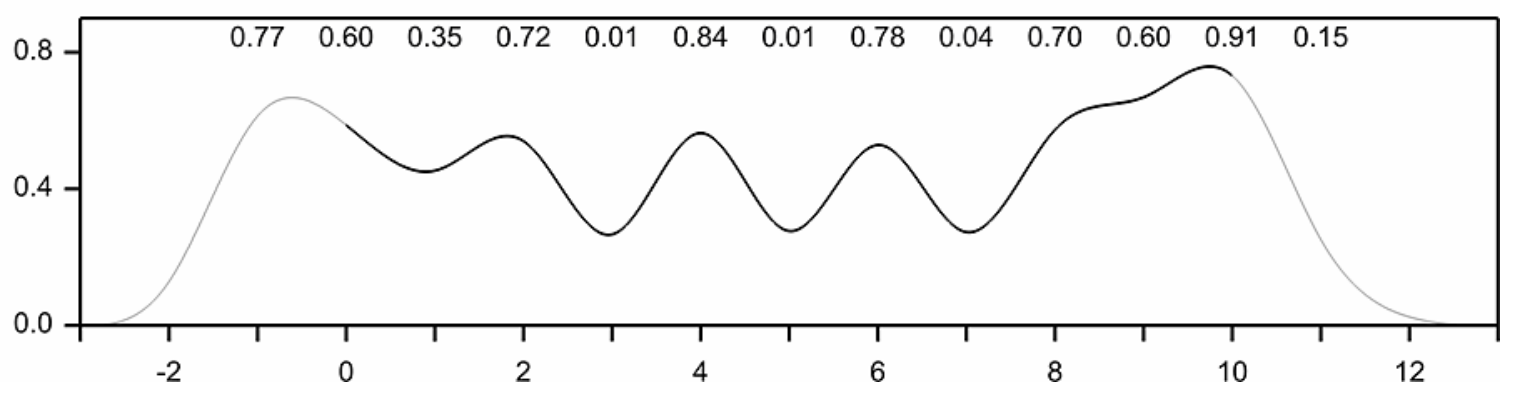

Figure 1. (a) Cubic B splines on the interval [0, 10] with equidistant knots at all integer values; (b) and (c) display linear combinations of these cubic $B$ splines with coefficients of the basis functions given at the top of the figures.

It is clear that B-splines form a very flexible family of curves encompassing smooth and ragged curves. Once the knots are given it is easy to compute the B splines and to fit a linear combination of B $\neg$ splines to data, e.g. using least squares. The choice of knots is crucial in successfully fitting B splines to data with underfitting and overfitting as potential pitfalls. Unfortunately, it is very hard to formulate automatic rules for knot placement [56]. Eilers \& Marx [51] therefore proposed that a relatively large number of equidistant knots be used, while applying a penalty on second order differences of the coefficients $\beta$ of adjacent B splines. The penalty they proposed equals $\sum_{j}\left(\beta_{j}-2 \beta_{j-1}+\beta_{j-2}\right)^{2}$ and this penalty has a value of 0.36 for Figure 1(b) and 15.38 for Figure 1(c). Clearly a small value for the sum of squared 
second order differences induces smooth curves while larger values give more ragged curves. A penalized spline with $\mathrm{k}$ equidistant knots can now be fitted to $\mathrm{m}$ data points $\left(y_{i}, x_{i}\right)$ by minimizing the penalized sums of squares.

$$
\sum_{i=1}^{m}\left\{y_{i}-\sum_{i=1}^{k} \beta_{j} B_{j}(x)\right\}^{\sim}+\lambda \sum_{i=3}^{k}\left(\beta_{j}-2 \beta_{j-1}+\beta_{j-2}\right)^{2}
$$

The first part of this expression is just the usual sums of squares. The parameter $\lambda$ offers continuous control over smoothness of the fit: large values of $\lambda$ will ensure that the sum of squared second order differences is small implying smoother curves. So instead of choosing the position of a number of knots, only a single penalty $\lambda$ needs to be chosen. The $\beta$ 's which minimize the penalized sums of squares are given by the set of linear equations $B^{T} y=\left(B^{T} B+\lambda D^{T} D\right) \beta$ where the elements of $\mathrm{B}$ are given by the $\mathrm{B}$ spline functions, and $\mathrm{D}$ is the matrix representation of the second order differences. The fitted values are then given by $B\left(B^{T} B+\lambda D^{T} D\right)^{-1} B^{T} y$ y which shows that the cubic spline smoother is linear in the observations y. By analogy with ordinary least squares the effective degrees of freedom of the smoother can be defined by the trace of the linear smoother $B\left(B^{T} B+\lambda D^{T} D\right)^{-1} B^{T}$. In order to fit a penalized spline with certain degrees of freedom, a search algorithm can be used to determine the associated penalty $\lambda$.

The discussion above is specific for least squares. We used logistic regression to fit penalized splines to species absence/presence data. Logistic regression, like every generalized linear model, can be fitted by means of iteratively reweighted least squares [57]. This can be used to generalize the fitting procedure above to logistic regression, see Eilers \& Marx [51] for details. Moreover, the effective degrees of freedom of the fitted spline is also available when employing logistic regression. It can, for example, be shown that one degree of freedom is equivalent to univariate logistic regression.

The only remaining issues are the number of equidistant knots and the choice of the penalty $\lambda$. The number of knots is not crucial, provided that there are not too few knots. We choose 39 equidistant knots, resulting in 40 intervals, between the minimum and maximum of the observed abiotic variable $\mathrm{x}$. Species response curves are fitted for degrees of freedom equal to $1,2, \ldots, 5$. The "best" curve was determined by backward deviance testing: the number of degrees of freedom was decreased one at a time, from 5 to 4,3 and 2, and this process was stopped when the resulting decrease in fit was significant at the $1 \%$ level as judged on the basis of a deviance test [54]. Special Fortran (employing algorithms from Press et al. [58]) and GenStat [59] programs to estimate the response curves were developed. A species response curves was only fitted when the species was present at minimally 25 relevés for which the abiotic soil variable was available.

The fitted penalized spline curve reflects the estimated parameter vector $\beta$. The standard errors of the estimate are however useless to assess the uncertainty of the fitted curve due to the induced penalty $\lambda$. Instead a $95 \%$ bootstrap interval of the fitted curve was obtained in the following way. A bootstrap sample with replacement, of the same size as the number of measurements, was drawn from the measurements and the penalized spline was fitted to the bootstrapped data. One thousand independent bootstrap samples were used to obtain 1000 estimated response curves. A $95 \%$ bootstrap interval is then given by the $2.5 \%$ and $97.5 \%$ percentiles of the fitted responses.

\section{2-4- Selection of Neophyte Species}

In this paper we define invasive species in the Netherlands as species that are given as neophytes in the Dutch flora [60]. A species is classified as a neophyte if it arrived in the country after 1825; it is classified as archaeophytic if it arrived and established a population in the Netherlands before that date. The year 1825 was chosen for practical reasons because from that date on reliable information is available and made available by Van der Meijden [60]. The species pool was split into two parts, neophytes and all other species. Species response curves were fitted for 981 species. 39 of these species are defined as neophytes by van der Meijden [60] and are present in the species pool, leaving 942 other species.

\section{2-5- Selection of Dutch Invasive Species}

Invasive Dutch species, which are indigenous in the Netherlands [60] and have become invasive in other parts of the world outside Europe, were selected from the IUCN invasive species list [61]. In total 52 species on the IUCN-list naturally occur in the Netherlands.

\section{2-6- Species Responses}

Means and standard errors of the range widths [50] were calculated for the neophytes and the other species group, the Dutch invasive species and the other species without the Dutch invasive species. A two sided 5\% student t-test was 
used to test for a difference between the averages of both groups for each abiotic soil parameter, assuming normal distribution. All calculations were carried out in GenStat [59].

\section{3- Discussion}

\section{3-1-Species Responses}

Responses were estimated for 981 species (39 neophytes and 942 other; Figure 2a-d gives an example of the range for four different species ). The indigenous species Cirsium dissectum (L.) Hill (meadow thistle, Figure 2a), has quite narrow ranges for most of the selected abiotic soil parameters (as expected for a rare species, see Wamelink et al., [45]. On the other hand Agrostis stolonifera L. (creeping bentgrass, Figure 2b), also an indigenous species, has rather wide ranges for most of the abiotic soil parameters. The neophytes Impatiens parviflora DC. (small balsam, Figure 2c) and Juncus tenuis Willd. (slender rush, Figure 2d) also show relatively wide ranges for at least part (I. parviflora) or most of the abiotic soil parameters (J. tenuis).

In following figures the bar per abiotic soil parameter gives the range based on the 5 and 95 percentile of the occurrence of the species. Each spoke of the wheel represents a different abiotic parameter. The values per parameter are standardised with the minimum absolute value set to $0 \%$ and the maximum value set at $100 \%$. The circles indicate the 0,20 th, 40th, 60th and 80th percentile of the species for the abiotic factor. The wheels make it possible to compare the ranges between the species. Below the wheels the abiotic soil parameters are given including the minimum and maximum value of the soil parameter of the 5 and 95 percentile ranges. With MSL: mean spring groundwater level; MHL: mean highest groundwater level; MLL: mean lowest groundwater level; moist: moist content and P; PO4. All scales are linear (though $\mathrm{pH}$ is the $10 \log$ of $\mathrm{H}+$ ).

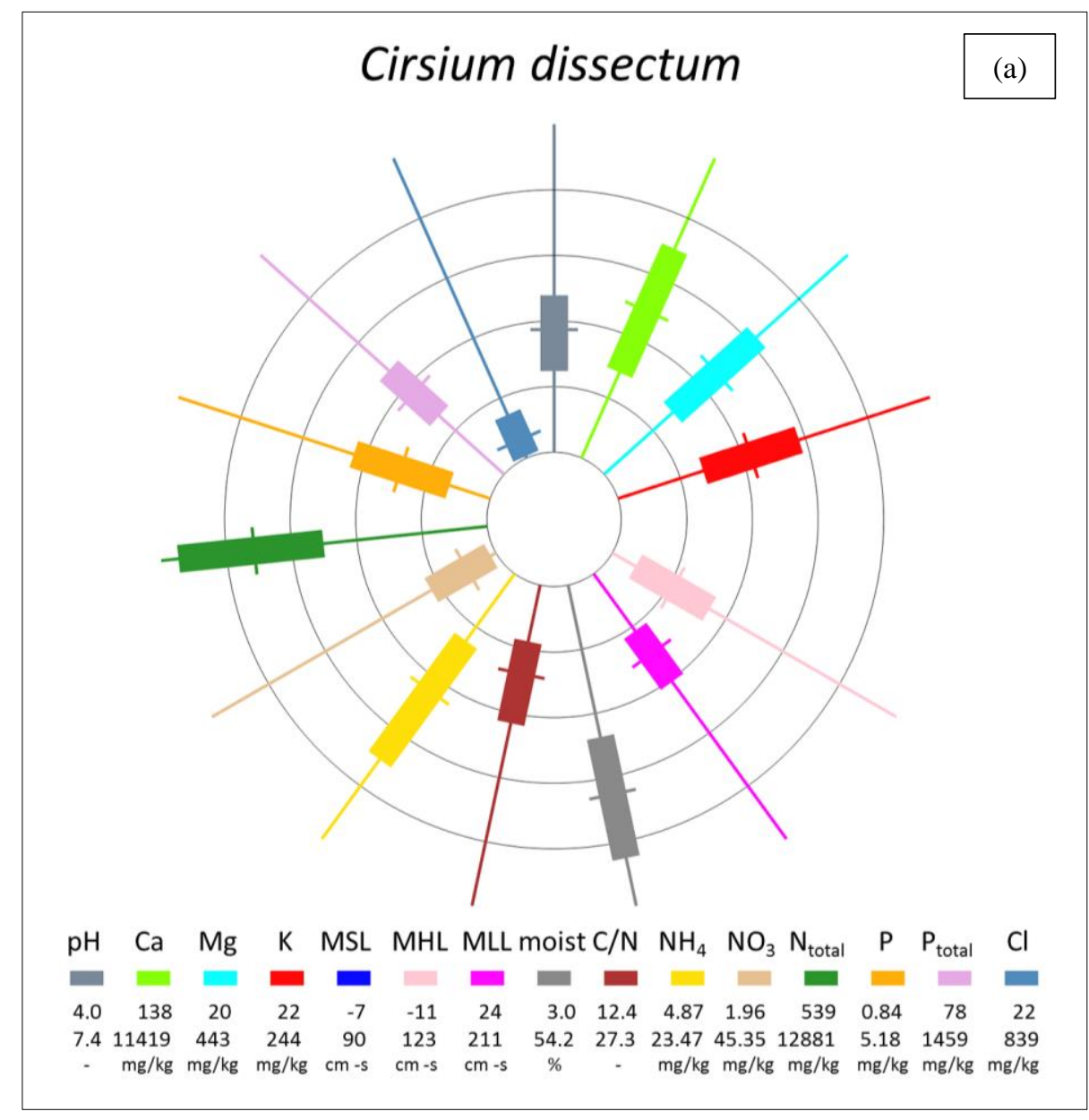



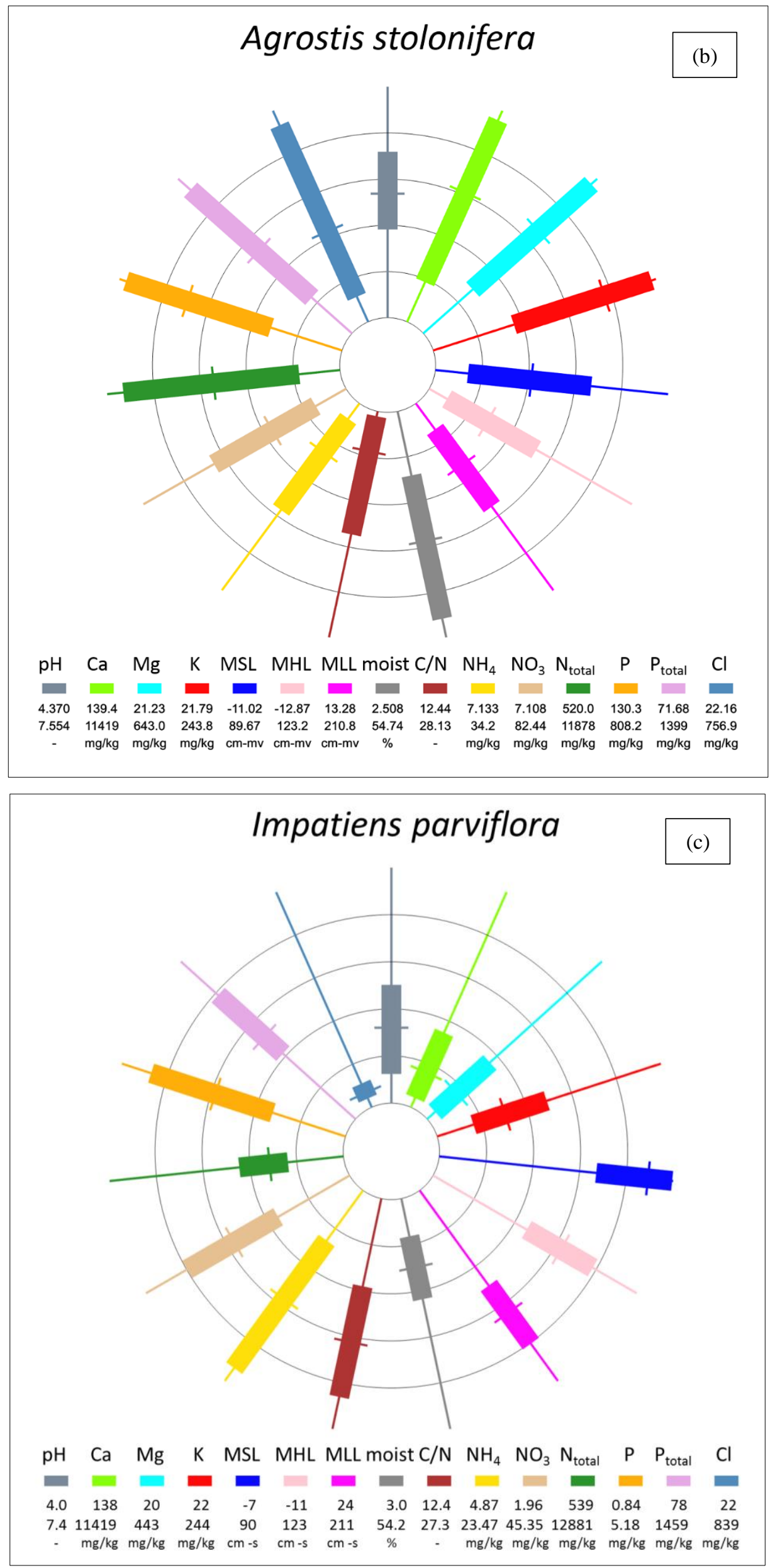


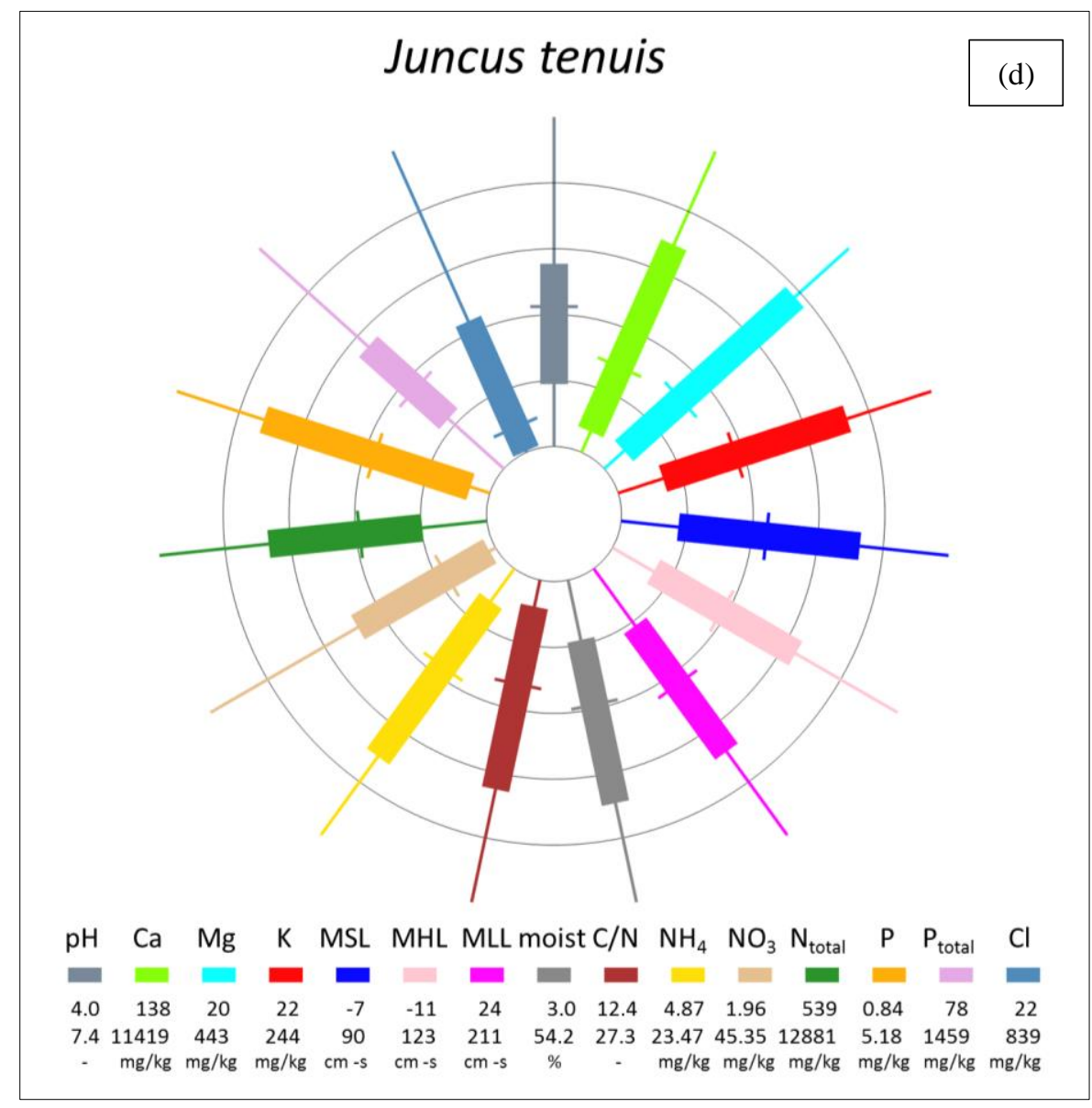

Figure 2. Abiotic ranges for the indigenous species Cirsium dissectum (L.) Hill (meadow thistle, Figure 2a), Agrostis stolonifera L. (creeping bentgrass, Figure 2b) and the neophytes Impatiens parviflora DC. (Small balsam, Figure 2c) and Juncus tenuis (slender rush, Figure 2d).

\section{3-2- Ranges of Neophyte Species}

Of the 16 abiotic soil parameters examined, six do not show a statistically significant difference in the range for neophytes and other species, two show significantly smaller ranges for neophytes and eight show significantly wider ranges for neophytes (Table 1). The two soil parameters that show narrower ranges for neophytes are calcium and chloride. The soil parameters that show wider ranges for neophytes are mostly related to nutrient availability: C/N, electric conductivity (EC), NH4, NO3, $\mathrm{N}$ total, $\mathrm{P}$ total, $\mathrm{P}$ and $\mathrm{pH}$. There is also a significant difference $(\mathrm{p}<0.05)$ for mean lowest groundwater level, but not for the other water related parameters mean spring groundwater level, mean highest groundwater level and moisture content of the soil.

Differences between the ranges are up to $32 \%(\mathrm{C} / \mathrm{N}$ and electric conductivity) and $38 \%(\mathrm{Cl})$; most of the differences are between 20 and $30 \%$.

Table 1. Species ranges for abiotic soil parameters for neophytes and other Dutch plant species, including standard error, the difference between both groups and the p-values for the difference (two tail student t-test, n.s.; not significant, $* \mathbf{p}<0.05$, $* * \mathbf{p}<\mathbf{0 . 0 1}, * * * \mathbf{p}<\mathbf{0 . 0 0 1})$. Results per species are given in Appendix 1 for neophytes and Appendix 2 for the 'other species'. $\mathrm{N}$ gives the number of entries of the parameter in the EC-database, i.e. the number of field measurements. With EC: Electric

Conductivity; MSL: mean spring groundwater level; MHL: mean highest groundwater level; MLL: mean lowest groundwater level (the latter three in $\mathrm{cm}$ below surface) and moisture: moisture content.

\begin{tabular}{|c|c|c|c|c|c|c|c|c|c|}
\hline & Unit & $\mathbf{n}$ & Other species & & Neophytes & & $\Delta$ & Against native & $\mathbf{p}$ \\
\hline & & & range & s.e. & range & s.e. & & $\%$ & \\
\hline $\mathrm{C} / \mathrm{N}$ & - & 2668 & 4.8 & 2.0 & 6.3 & 2.0 & 1.5 & 32 & $0.000^{* * *}$ \\
\hline $\mathrm{Ca}^{1}$ & $\mathrm{mg} / \mathrm{kg}$ & 758 & 5053.5 & 1985.7 & 4075.2 & 2153.2 & -978.3 & -19 & $0.003^{* *}$ \\
\hline $\mathrm{Cl}$ & $\mathrm{mg} / \mathrm{kg}$ & 851 & 204.5 & 201.5 & 127.4 & 127.0 & -77.1 & -38 & $0.018^{*}$ \\
\hline $\mathrm{EC}$ & $\mathrm{mS}$ & 545 & 504.6 & 207.2 & 664.2 & 202.0 & 159.6 & 32 & $0.000^{* * *}$ \\
\hline
\end{tabular}




\begin{tabular}{|c|c|c|c|c|c|c|c|c|c|}
\hline $\mathrm{K}^{1}$ & $\mathrm{mg} / \mathrm{kg}$ & 737 & 99.4 & 31.4 & 93.6 & 37.4 & -5.7 & & $0.269^{\text {n.s. }}$ \\
\hline $\mathrm{Mg}^{1}$ & $\mathrm{mg} / \mathrm{kg}$ & 1273 & 150.3 & 63.7 & 138.8 & 66.8 & -11.5 & & $0.269^{\text {n.s. }}$ \\
\hline MHL & $\mathrm{cm}-\mathrm{bs}$ & 1873 & 47.8 & 17.2 & 48.5 & 13.9 & 0.8 & & $0.783^{\text {n.s. }}$ \\
\hline MLL & $\mathrm{cm}-\mathrm{bs}$ & 1808 & 57.1 & 23.1 & 65.8 & 19.7 & 8.7 & 15 & $0.020^{*}$ \\
\hline MSL & $\mathrm{cm}-\mathrm{bs}$ & 1168 & 39.2 & 12.2 & 41.1 & 12.9 & 1.9 & & $0.339^{\text {n.s. }}$ \\
\hline Moisture & $\%$ & 1785 & 19.2 & 7.1 & 18.3 & 8.4 & -0.8 & & $0.479^{\text {n.s. }}$ \\
\hline $\mathrm{NH}_{4}^{2}$ & $\mathrm{mg} / \mathrm{kg}$ & 1493 & 6.4 & 2.6 & 7.9 & 3.0 & 1.6 & 24 & $0.000^{* * *}$ \\
\hline $\mathrm{NO}_{3}{ }^{2}$ & $\mathrm{mg} / \mathrm{kg}$ & 1492 & 18.4 & 7.4 & 22.8 & 7.0 & 4.4 & 24 & $0.000^{* * *}$ \\
\hline $\mathrm{N}_{\text {total }}$ & $\mathrm{mg} / \mathrm{kg}$ & 3187 & 3824.6 & 1786.7 & 3590.0 & 1963.9 & -234.6 & & $0.424^{\text {n.s. }}$ \\
\hline $\mathrm{PO}_{4}{ }^{2}$ & $\mathrm{mg} / \mathrm{kg}$ & 1461 & 1.5 & 0.7 & 2.0 & 0.6 & 0.4 & 29 & $0.000^{* * *}$ \\
\hline$P_{\text {total }}$ & $\mathrm{mg} / \mathrm{kg}$ & 3169 & 416.6 & 165.4 & 501.2 & 216.4 & 84.7 & 20 & $0.002^{* *}$ \\
\hline $\mathrm{pH}^{1}$ & - & 5914 & 1.1 & 0.4 & 1.4 & 0.4 & 0.3 & 25 & $0.000^{* * *}$ \\
\hline
\end{tabular}

\section{3-3- Range of Dutch Invasive Species}

The ranges of species that are indigenous for the Netherlands and are listed in the IUCN invasive species list show a result that differs in several aspects from the neophytes (Table 2). For all abiotic factors there is a wider range for the Dutch invasive species. However, most of the differences are not significant; for only seven out of the sixteen abiotic soil parameters there is a significant difference at the $5 \%$ level: $\mathrm{C} / \mathrm{N}, \mathrm{Cl}, \mathrm{K}$, mean highest and lowest groundwater level, $\mathrm{P}$ total and $\mathrm{pH}$. The differences between the invasive and the non-invasive Dutch species are generally smaller than between the neophytes and the other Dutch species. The highest difference is 33\% (also for $\mathrm{Cl}$ ), but most differences are below $20 \%$.

Table 2. Species ranges for abiotic soil parameters Dutch other species (without the neophytes and without the Dutch invasive species) and Dutch invasive species, including standard error, the difference between both groups and the p-values for the difference (two tail student t-test, n.s.; not significant, ${ }^{*} \mathbf{p}<0.05, * * \mathbf{p}<0.01, * * * \mathbf{p}<0.001$ ). Results per species are given in Appendix 3 for Dutch invasive species and Appendix 4 for the 'Dutch other species'. With EC: Electric

Conductivity; MSL: mean spring groundwater level; MHL: mean highest groundwater level; MLL: mean lowest groundwater level (the latter three in $\mathrm{cm}$ below surface) and moisture: moisture content.

\begin{tabular}{|c|c|c|c|c|c|c|c|c|}
\hline Parameter & Unit & Other species & & Dutch invasive species & & $\Delta$ & Against native & $\mathbf{p}$ \\
\hline & & range & s.e. & range & s.e. & & $\%$ & \\
\hline $\mathrm{C} / \mathrm{N}$ & - & 4.7 & 2.0 & 5.8 & 2.0 & 1.0 & 22 & $0.000^{* * *}$ \\
\hline $\mathrm{Ca}^{1}$ & $\mathrm{mg} / \mathrm{kg}$ & 5036.8 & 1991.8 & 5544.9 & 1894.0 & 508.1 & 10 & $0.071^{\text {n.s. }}$ \\
\hline $\mathrm{Cl}$ & $\mathrm{mg} / \mathrm{kg}$ & 201.8 & 201.7 & 269.0 & 199.0 & 67.3 & 33 & $0.018^{*}$ \\
\hline $\mathrm{EC}$ & $\mathrm{mS}$ & 503.4 & 208.1 & 523.0 & 186.4 & 19.6 & 4 & $0.503^{\text {n.s. }}$ \\
\hline $\mathrm{K}^{1}$ & $\mathrm{mg} / \mathrm{kg}$ & 98.6 & 31.3 & 117.8 & 30.9 & 19.2 & 19 & $0.000^{* * *}$ \\
\hline $\mathrm{Mg}^{1}$ & $\mathrm{mg} / \mathrm{kg}$ & 149.9 & 64.1 & 159.7 & 55.5 & 9.7 & 6 & $0.281^{\text {n.s. }}$ \\
\hline MHL & $\mathrm{cm}-\mathrm{bs}$ & 47.4 & 17.2 & 55.5 & 13.8 & 8.1 & 17 & $0.000^{* * *}$ \\
\hline MLL & $\mathrm{cm}-\mathrm{bs}$ & 56.7 & 23.3 & 65.7 & 17.8 & 9.1 & 16 & $0.006^{* *}$ \\
\hline MSL & $\mathrm{cm}-\mathrm{bs}$ & 39.1 & 12.3 & 41.9 & 10.5 & 2.8 & 7 & $0.107^{\text {n.s. }}$ \\
\hline Moisture & $\%$ & 19.1 & 7.1 & 20.9 & 5.3 & 1.8 & 9 & $0.078^{\text {n.s. }}$ \\
\hline $\mathrm{NH}_{4}^{2}$ & $\mathrm{mg} / \mathrm{kg}$ & 6.4 & 2.6 & 6.8 & 2.4 & 0.4 & 7 & $0.236^{\text {n.s. }}$ \\
\hline $\mathrm{NO}_{3}{ }^{2}$ & $\mathrm{mg} / \mathrm{kg}$ & 18.3 & 7.4 & 20.1 & 7.0 & 1.8 & 10 & $0.087^{\text {n.s. }}$ \\
\hline $\mathrm{N}_{\text {total }}$ & $\mathrm{mg} / \mathrm{kg}$ & 3817.6 & 1803.8 & 4108.3 & 1552.7 & 290.8 & 8 & $0.251^{\text {n.s. }}$ \\
\hline $\mathrm{PO}_{4}{ }^{2}$ & $\mathrm{mg} / \mathrm{kg}$ & 1.5 & 0.7 & 1.6 & 0.6 & 0.1 & 5 & $0.446^{\text {n.s. }}$ \\
\hline $\mathrm{P}_{\text {total }}$ & $\mathrm{mg} / \mathrm{kg}$ & 414.3 & 167.4 & 470.2 & 128.2 & 55.9 & 13 & $0.017^{*}$ \\
\hline $\mathrm{pH}^{1}$ & - & 1.1 & 0.4 & 1.4 & 0.4 & 0.3 & 26 & $0.000^{* * *}$ \\
\hline
\end{tabular}




\section{4-Discussion}

We investigated the possibility that broader abiotic soil preferences could be one of the factors that may determine the success of neophytes. Probably the first factor is efficient dispersal followed by the availability of an occupying empty places in the vegetation. After the seed has arrived there is an establishment phase where abiotic preferences play a major role. Invasive species may even alter abiotic conditions depending on the initial conditions and thus enhance their own success $[10,62]$. All these process may interact with each other. We only investigated one of these factors; the influence of abiotic preference of neophytes and its effect on the species success.

Our results agree with the extensive research on F. japonica by Beerling et al. [63]. This neophyte, which was not included in our research, has wide ranges for soil $\mathrm{pH}$ and for nutrient availability (see their Table 1). F. japonica was not included in our study because there are not enough field measurements available yet to estimate the response function and thus the abiotic ranges. Galatowitsch et al. [64] note that one of the main reasons for the success of invasive species is their ability to better cope with local circumstances than indigenous species (i.e. the environmental constraints hypothesis). Our results show that invasive species have wider ranges for important abiotic soil parameters and this may partly explain why they are able to better cope with local circumstances. The success of invasive species in the riparian Central Grasslands of the USA was in the first instance found to be influenced by soil parameters [65]. In contrast to rare species, the neophytes show both narrower and wider ranges than other species, where the rare species only had narrower ranges [45]. Narrower ranges for neophytes occur for chloride and calcium. This may be associated with saline conditions. Species with wide ranges for these two elements belong to the small group of species that is adapted to the stressful circumstances of saline soils [66]. It therefore seems logical that not many neophytes can tolerate high salt conditions. Consequently, the group of neophytes is likely to show narrower ranges for at least chloride.

For practical reasons our definition of neophytes as species that arrived after 1825, mainly follows the Dutch flora; however, our list of neophytes also includes some species that the Flora classifies as archeophytic, such as Castanea sativa Mill. (Sweet chestnut).

Dutch species that became invasive outside Europe show comparable results to those for the neophytes in the Netherlands. For some abiotic soil parameters, the Dutch invasive species had wider ranges. Thus, some of the soil parameters, the ones that show a wider range, may indicate the potential increase of a species' invasiveness. However, while the ranges for nutrient related parameters for the neophytes showed significant differences, this is not the case for Dutch invasive species, which show differences for $\mathrm{pH}$, groundwater table and for only $\mathrm{P}$ total and potassium. This difference may partly be due to special Dutch and European circumstances, where many natural areas are polluted with nitrogen or phosphorus [67-69]. Pollution creates new circumstances and thus opportunities for indigenous but also for invasive species. If the world wide increase of nitrogen deposition will continue (as predicted by Galloway et al. [68]) we expect new abiotic circumstances will also increase the threat of invasive species coming from outside Europe. Increased nitrogen deposition may therefore not only directly affect species composition $[45,70]$ but also indirectly via the increased threat of invasive species. The evidence that species with wide ranges for $\mathrm{pH}$ and groundwater table have been particularly successful as neophytes coming from outside Europe, agrees with the idea that these parameters largely determine species occurrence under less polluted circumstances.

By estimating the plant species' abiotic ranges it may be possible to predict whether a species may become invasive worldwide. This has already been proposed by [71], who looked at the temperature preferences of invasive species in South Africa, and found that this abiotic parameter can be used to assess the potency of invasive species worldwide. The method used here, of assessing species' abiotic preferences, could be one of the new techniques for risk assessment as suggested by the Ecological Society of America [72].

The results can be used in all of the three stages mentioned in the EU regulation for invasive alien species [21]. The abiotic preferences of species, if known, may partly help to assess which species may become invasive. Upon the arrival of new species an assessment of their abiotic preferences may be part of an early warning system. A rapid response may then possible, for instance via management or a ban on the import of the species. Existing problems with invasive species may also be solved via management based on knowledge of their abiotic preferences.

We hypothesized that part of the success of invasive species may be their adaptation to a wide range of abiotic soil circumstances. This is indeed the case for part of the examined parameters, mostly related to nutrient availability and soil $\mathrm{pH}$. This indicates that the success of invasive species is, at least partly, related to their ability to grow under a wide range of nutrient availability and soil $\mathrm{pH}$. Their success may also be stimulated by the increasing pollution of natural areas by excessive nitrogen (via deposition, [67, 73]. The control of invasive species that become pests, such as F. japonica or A. platanoides, may therefore be found through resolving the broader problem of eutrophication of the natural environment. 


\section{5- Acknowledgments}

Most of the data was collected in the field by others, to whom we are greatly indebted. We thank the various reviewers for their comments and input.

\section{6- Funding}

This research was funded by the ministry of Agriculture, Nature and Food Quality.

\section{7- Conflict of Interest}

The authors declare no conflict of interest.

\section{8- References}

[1] Levin, S.A. "The Problem of Pattern and Scale in Ecology: The Robert H. MacArthur Award Lecture.” Ecology 73 (1992): 1943 1967. doi: $10.2307 / 1941447$.

[2] Mack, R.N., D. Simberloff, W.M. Lonsdale, H. Evans, M. Clout and F.A. Bazzaz. "Biotic invasions: causes, epidemiology, global consequences, and control.” Ecological Letters 10 (2000): 689-710. doi: 10.2307/2641039.

[3] Strayer, D.L. "Alien species in fresh waters: ecological effects, interactions with other stressors, and prospects for the future." Freshwater Biology 55 (2010): 152-174. doi: 10.1111/j.1365-2427.2009.02380.x.

[4] Leuven, R.S.E.W., G. van der Velde, I. Baijens, J. Snijders, C. van der Zwart, H.J.R. Lenders, and A. bij de Vaate. "The river Rhine: a global highway for dispersal of aquatic invasive species." Biological Invasions 11 (2009): 1989-2008. doi: 10.1007/s10530-009-9491-7.

[5] Gilbert, N., K. Myers, B.D. Cooke, J.D. Dunsmore, P.J. Fullagar, J.A. Gibb, D.R. King, I. Parer, S.H. Wheeler, and D.H. Wood. “Comparative dynamics of Australasian rabbit populations." Aust. Wild Res. 14 (1987): 491-503. doi: 10.1071/wr9870491.

[6] Pech, R.P., A.R.E. Sinclair, A.E. Newsome and P.C. Catling,. "Limits to Predator Regulation of Rabbits in Australia: Evidence from Predator-Removal Experiments.” Oecologia 89 (1992): 102-112. doi: 10.1007/bf00319021.

[7] Blackburn, T. M., P. Cassey, R.P.K. Duncan, L. Evans and K.J. Gaston. "Avian extinction and mammalian introductions on oceanic islands.” Science 305 (2004): 1955-1958. doi: 10.1126/science.1107333.

[8] Chynoweth, M.W., C.M. Litton, C.A. Lepczyk, S.C. Hess and S. Cordell. "Biology and Impacts of Pacific Island Invasive Species. 9. Capra hircus, the Feral Goat (Mammalia: Bovidae).” Pacific Science, 67 (2013): 141-156. doi: 10.2984/67.2.1.

[9] Stinson, K.A., S.A. Campbell, J.R. Powell, B.E. Wolfe, R.M. Callaway, G.C. Thelen, S.G. Hallett, D. Prati and J.N. Klironomos,. "Invasive plant suppresses the growth of native tree seedlings by disrupting belowground mutualisms." PLoS Biology, 4 (2006): 727-731. doi: 10.1371/journal.pbio.0040140.

[10] Vanderhoeven, S., N. Dassonville, and P. Meerts,. "Increased Topsoil Mineral Nutrient Concentrations Under exotic invasive plants in Belgium.” Plant Soil, 275 (2005): 169-179. doi: 10.1007/s11104-005-1257-0.

[11] Hejda, M. and P. Pysek. "What is the impact of Impatiens glandulifera on species diversity of invaded riparian vegetation?" Biological Conservation, 132 (2006): 143-152. doi: 10.1016/j.biocon.2006.03.025.

[12] Diekmann, M., H. Effertz, M. Baranowski, and C. Dupré. "Weak effects on plant diversity of two invasive Impatiens species." Plant Ecology 217 (2016): 1503-1514. doi: 10.1007/s11258-016-0663-0.

[13] Harkel, M.J.,F. ten and van der Meulen. "Impact of Grazing and Atmospheric Nitrogen Deposition on the Vegetation of Dry Coastal Dune Grasslands.” Journal of Vegetation Science, 7 (1996): 445-452. doi: 10.2307/3236288.

[14] Thompson, H.V. and C.M. King. "The European rabbit: the history and biology of a successful colonizer." (1994).

[15] Isermann, M. "Expansion of Rosa rugosa and Hippophaë rhamnoides in coastal grey dunes: Effects at different spatial scales." Flora: Morphology, Distribution, Functional Ecology of Plants 203 (2008): 273-280. doi: 10.1016/j.flora.2007.03.009.

[16] EU. 2017a. http://ec.europa.eu/environment/nature/invasivealien/list/index_en.htm. Last visited 21-7-2018.

[17] EU. 2017b. http://eur-lex.europa.eu/legal-content/EN/TXT/?uri=OJ:JOL_2014_317_R_0003. Last visited 21-7-2018.

[18] Maerz, J.C., B. Blossey and V. Nuzzo. “Green frogs show reduced foraging success in habitats invaded by Japanese knotweed.” Biodiversity and Conservation 14 (2005): 2901-2911. doi: 10.1007/s10531-004-0223-0.

[19] Maskell, L.C., L.G. Firbank, K. Thompson, J.M. Bullock and S.M. Smart. "Interactions between non-native plant species and the floristic composition of common habitats." Journal of Ecology 94 (2006): 1052-1060. doi: 10.1111/j.1365- 
2745.2006.01172.x.

[20] Essl, F., S. Dullinger and I. Kleinbauer. "Changes in the spatio-temporal patterns and habitat preferences of Ambrosia artemisiifolia during its invasion of Austria." Preslia 81 (2009): 119-133.

[21] EU, 2015. http://ec.europa.eu/environment/nature/invasivealien/index_en.htm. Last visited 21-7-2018.

[22] Crawley, M.J. "Natural Enemies: the Population Biology of Predators, Parasites, and Diseases." (1992). doi: $10.1002 / 9781444314076$.

[23] Goeden, R.D. and L.A. Andres. "Biological Control of Weeds in Terrestrial and Aquatic Environments." In: Handbook of Biological Control (eds. Bellows, T.S. and Fisher, T.W.) pp 871-890. (1999). doi: 10.1016/b978-012257305-7/50081-3.

[24] Mitchell, C.G. and A.G. Power. "Release of invasive plants from fungal and viral pathogens." Nature 421 (2003): 625-627. doi: 10.1038/nature01317.

[25] Wardle, D.A., R.D. Bardgett, R.M. Callaway and W.H. Van Der Putten. "Terrestrial ecosystem responses to species gains and losses.” Science 332 (2011): 1273-1277. doi: 10.1126/science.1197479.

[26] Simberloff, D. and B. Von Holle. "Positive interactions of nonindigenous species: invasional meltdown?” Biol. Invas. 1 (1999): 21-31. doi: 10.1023/a:1010086329619.

[27] Richardson, D.M., N. Allsopp, C.M. D’Antonio, S.J. Milton, and M. Rejmanek. "Plant invasions—-the role of mutualisms." Biol. Rev. 75 (2000): 65-93. doi: 10.1111/j.1469-185x.1999.tb00041.x.

[28] Marler, M.M., C.A. Zabinski and R.M. Callaway. "Mycorrhizae indirectly enhance competitive effects of an invasive forb on a native bunchgrass.” Ecology 80 (1999): 1180-1186. doi: 10.2307/177065.

[29] Callaway, R.M., B. Newingham, C.A. Zabinski, and B.E. Mahall. "Compensatory growth and competitive ability of an invasive weed are enhanced by soil fungi and native neighbours.” Ecol. Lett. 4 (2001): 1-5. doi: 10.1046/j.1461-0248.2001.00251.x.

[30] Klironomos, J. "Feedback with soil b iota contributes to plant rarity and invasiveness in communities." Nature 417 (2002): 6770. doi: $10.1038 / 417067$ a.

[31] Callaway, R.M., G.C. Thelen, A. Rodriguez, and W.E. Holben. "Soil biota and exotic plant invasion." Nature 427 (2004). 731 733. doi: $10.1038 /$ nature 02322 .

[32] Broennimann O., U.A. Treier, H. Müller-Schärer, W. Thuiller, A.T. Peterson and A. Guisan. "Evidence of climatic niche shift during biological invasion.” Ecol. Lett. 10 (2007): 701-709. doi: 10.1111/j.1461-0248.2007.01060.x.

[33] Maron, J.L., M. Vilà, R. Bommarco, S. Elmendorf and P. Beardsley. "Rapid evolution of an invasive plant." Ecological Monographs 74 (2004): 261-280. doi: 10.1890/03-4027.

[34] Thomas, C.D., A.Cameron, R.E. Green, M. Bakkenes, L.J. Beaumont, Y.C. Collingham, B.F.N. Erasmus, M.F. de Siqueira, A. Grainger, L. Hannah, L. Hughes, B. Huntley, A.S. van Jaarsveld, G.F. Midgley, L. Miles, M.A. Ortega-Huerta, A. Townsend Peterson, O.L. Phillips and S.E. Williams. "Extinction risk from climate change." Nature 427 (2004): 145-148. doi: 10.1038/nature02121.

[35] Luque, G.M., C. Bellard, C. Bertelsmeier, E. Bonnaud, P. Genovesi, D. Simberloff and F. Courchamp. "The 100th of the world's worst invasive alien species.” Biological Invasions 16 (2014): 981-985. doi: 10.1007/s10530-013-0561-5.

[36] Vitousek P.M. "Biological invasions and ecosystem processes: towards an integration of population biology and ecosystem studies.” Oikos, 57 (1990): 7-13. doi: 10.2307/3565731.

[37] Ehrenfeld, J.G. "Effects of exotic plant invasions on soil nutrient cycling processes." Ecosystems 6 (2003): 503-23. doi: $10.1007 / \mathrm{s} 10021-002-0151-3$.

[38] Crooks J.A. "Characterizing ecosystem-level consequences of biological invasions: the role of ecosystem engineers." Oikos 97 (2002): 153-66. doi: 10.1034/j.1600-0706.2002.970201.x.

[39] Laughlin, D.C. and S.R. Abella. "Abiotic and biotic factors explain independent gradients of plant community composition in ponderosa pine forests.” Ecological Modelling, 205 (2007): 231-240. doi: 10.1016/j.ecolmodel.2007.02.018.

[40] Foley, J.A., R. DeFries, G.P. Asner, C. Barford, G. Bonan, S.R. Carpenter, F.S. Chapin, M.T. Coe1, G.C. Daily, H.K. Gibbs, J.H. Helkowski, T. Holloway, E.A. Howard, C.J. Kucharik, C. Monfreda, J.A. Patz, I.C. Prentice, N. Ramankutty, and P.K. Snyder. "Global Consequences of Land Use". Science 309 (2005): 570-574. doi: 10.1126/science.1111772.

[41] Fleischner, T.L. "Ecological Costs of Livestock Grazing in Western North America." Conservation Biology 8 (1994): $629-644$. doi: 10.1046/j.1523-1739.1994.08030629.x.

[42] Ripple, W.J. and R.L. Beschta. Trophic cascades in Yellowstone: The first 15 years after wolf reintroduction.” Biological Conservation, 145 (2012): 205-213. doi: 10.1016/j.biocon.2011.11.005. 
[43] Ozinga, W.A., J.H.J. Schaminée, R.M. Bekker, S. Bonn, P. Poschlod, O. Tackenberg, J. Bakker and J.M. van Groenendael. "Predictability of plant species composition from environmental conditions is constrained by dispersal limitation." Oikos, 108 (2005): 555-561. doi: 10.1111/j.0030-1299.2005.13632.x.

[44] Normand S., R.E. Ricklefs, F. Sko, J. Bladt, O. Tackenberg and J.C. Svenning. "Postglacial migration supplements climate in determining plant species ranges in Europe.” Proceedings of the Royal Society B: Biological Sciences 278 (2011): $3644-3653$. doi: $10.1098 / \mathrm{rspb} .2010 .2769$.

[45] Wamelink, G.W.W., P.W. Goedhart and J.Y. Frissel. "Why Some Plant Species Are Rare.” PLoS ONE 9(7) (2014). e102674. doi:10.1371/journal.pone.0102674.

[46] Wamelink, G.W.W., M.H.C. van Adrichem, H.F. van Dobben, J.Y. Frissel, M. Held, den, V. Joosten, A.H. Malinowska, P.A. Slim, and R.M.A. Wegman. "Vegetation relevés and soil measurements in the Netherlands; a database." Biodiversity and Ecology 2012 (2012): 125-132. doi: 10.7809/b-e.00067.

[47] Schaminee, J.H.J., S.M. Hennekens and W. Ozinga. “The Dutch national vegetation database.” Biodiversity and Ecology 2012 (2012): 201-209. doi: 10.7809/b-e.00077.

[48] Braun-Blanquet J. ”Pflanzensoziologie: Grundzüge der Vegetationskunde.” 3. ed. (1964).

[49] Schaminée, J.H.J., Stortelder, A.H.F., Westhoff, V. 1995. De Vegetatie van Nederland; deel 1: Inleiding tot de plantensociologie - grondslagen, methoden en toepassingen. Opulus Press, Uppsala.

[50] Wamelink, G.W.W., P.W. Goedhart, H.F. van Dobben and F. Berendse. "Plant species as predictors of soil pH: replacing expert judgement by measurements.” Journal of vegetation science 16 (2005): 461-470. doi: 10.1214/ss/1038425655.

[51] Eilers, H.C. and B.D. Marx. "Flexible smoothing with Bsplines and penalties." Statistical Science, 11 (1996): 89-121. doi: $10.1214 / \mathrm{ss} / 1038425655$.

[52] Wamelink, G.W.W., P.W. Goedhart, A.H. Malinowska, J.Y. Frissel, R.J.M. Wegman, P.A. Slim and H.F. van Dobben. "Ecological ranges for the $\mathrm{pH}$ and NO3 of syntaxa: A new basis for the estimation of critical loads for acid and nitrogen deposition.” Journal of Vegetation Science 22 (2011): 741-749.

[53] Hastie, T. and R. Tibshirani. "Generalized additive models.” (1990). doi: 10.1002/0471667196.ess0297.pub2.

[54] De Boor, C. “A practical guide to splines.” (1978). doi: 10.1007/978-1-4612-6333-3.

[55] Wold, S. "Spline functions in data-analysis." Technometrics, 16 (1974): 1-11. doi: 10.2307/1267485.

[56] McCullagh, P. and J.A. Nelder. Generalized linear models, second edition. (1989. doi: 10.1007/978-1-4899-3242-6.

[57] Press, W.H., B.P. Flannery, S.A. Teukolsky and W.T. Vetterling. "Numerical recipes. The art of scientific computing (FORTRAN version).” (1989).

[58] VSN International. “Genstat for Windows.” 18th Edition. (2015). http://www.vsni.co.uk/

[59] Meijden, R. Van der. "Heukels flora van Nederland.” 23rd Edition. (2005).

[60] Luque, G.M. (2014). http://www.issg.org/database/species/. Last visited 21-7-2018.

[61] Dassonville, N., S. Vanderhoeven, V. Vanparys, M. Hayez, W. Gruber, and P. Meerts. "Impacts of alien invasive plants on soil nutrients are correlated with initial site conditions in NW Europe.” Oecologia, 157 (2008): 131-140. doi: 10.1007/s00442-0081054-6.

[62] Beerling D.J., J.P. Bailey and A.P. Conolly. “Fallopia japonica (Houtt.) Ronse Decraene.” J. Eco.1, 82 (1994): $959-79$. doi: $10.2307 / 2261459$.

[63] Galatowitsch, S.M., N.O. Anderson and P.D. Ascher. "Invasiveness in wetland plants in temperate North America." Wetlands 19 (1999): 733-755. doi: 10.1007/bf03161781.

[64] Stohlgren T.J., K.A. Bull, Y. Otsuki, C.A. Villa and M. Lee. "Riparian zones as havens for exotic plant species in the central grasslands.” Plant Ecology 138 (1998): 113-125. doi: 10.1023/a:1009764909413.

[65] Flowers, T.J. and T.D. Colmer. "Salinity Tolerance in Halophytes.” New Phytologist, 179 (2008): 945-963. doi: 10.1111/j.14698137.2008.02531.x.

[66] Galloway, J.N., J.D. Aber, J.W. Erisman, S.P. Seitzinger, R.W. Howarth, E.B. Cowling, and B.J. Cosby. "The Nitrogen Cascade." Bioscience, 53 (2003): 341-356. doi: 10.1641/0006-3568(2003)053[0341:tnc]2.0.co;2.

[67] Galloway, J.N., F.J. Dentener, D.G. Capone, E.W. Boyer, R.W. Howarth, S.P. Seitzinger, G.P. Asner, C.C. Cleveland, P.A. Green, E.A. Holland, D.M. Karl, A.F. Michaels, J.H. Porter, A.R. Townsend, and C.J. Vörösmarty. "Nitrogen cycles: Past, present, and future." Biogeochemistry 70 (2004): 153-226. doi: 10.1007/s10533-004-0370-0. 
[68] Haygarth, P.M., L.M. Condron, A.L.Heathwaite, B.L. Turner and G.P. Harris. "The phosphorus transfer continuum: Linking source to impact with an interdisciplinary and multi-scaled approach." Science of The Total Environment 344 (2005): 5-14. doi: 10.1016/j.scitotenv.2005.02.001.

[69] Bobbink, R., M. Hornung, and J.G.M. Roelofs. "The effects of air-borne nitrogen pollutants on species diversity in natural and semi-natural European vegetation.” J. Ecol, 86 (1998): 717-738. doi: 10.1046/j.1365-2745.1998.8650717.x.

[70] Thuiller, W., D.M. Richardson, P. Pyssek, G.F. Midgley, G.O. Hughes, and M. Rouget. "Niche-based modelling as a tool for predicting the risk of alien plant invasions at a global scale." Global Change Biology 11 (2005): 2234-2250. doi: 10.1111/j.13652486.2005.001018.x.

[71] Lodge, D.M., S. Williams, H.J. MacIsaac, K.R. Hayes, B. Leung, S. Reichard, R.N. Mack, P.B. Moyle, M. Smith, D.A. Andow, J.T. Carlton, and A. McMichael. "Biological invasions: Recommendations for U.S. policy and management." Ecological Applications 16 (2006): 2035-2054. doi: 10.1890/1051-0761(2006)016[2035:birfup]2.0.co;2.

[72] Fagerli, H., M. Gauss, A. Benedictow, J. Griesfeller, J.E. Jonson, A. Nyiri, M. Schulz, D. Simpson, B.M. Steensen, S. Tsyro, A. Valdebenito, P. Wind, W. Aas, A. Hjellbrekke, K. Mareckova, R. Wankmuller, T. Iversen, A. Kirkevag, O. Seland and M. Vieno. "Transboundary acidification, eutrophication and ground level ozone in Europe in 2009." EMEP Report 1/2011. (2010). 


\begin{tabular}{|c|c|c|c|c|c|c|c|c|c|c|c|c|c|c|c|c|c|}
\hline Species name & neophytes (0)/ivasive (1) & $\begin{array}{c}\mathrm{C} / \mathrm{N} \\
-\end{array}$ & $\begin{array}{c}\mathrm{Ca}(\mathrm{H} 20) \\
\mathrm{mg} / \mathrm{kg}\end{array}$ & $\begin{array}{c}\mathrm{Cl} \\
\mathrm{mg} / \mathrm{kg}\end{array}$ & $\begin{array}{l}\text { Electronic conductivity } \\
\mathrm{mS}\end{array}$ & $\begin{array}{c}\mathrm{K}(\mathrm{H} 20) \\
\mathrm{mg} / \mathrm{kg}\end{array}$ & $\begin{array}{c}\mathrm{Mg}(\mathrm{H} 20) \\
\mathrm{mg} / \mathrm{kg}\end{array}$ & $\begin{array}{l}\text { MHL } \\
\mathrm{cm}-\mathrm{s}\end{array}$ & $\begin{array}{l}\text { MLL } \\
\mathrm{cm}-\mathrm{s}\end{array}$ & $\begin{array}{l}\text { MSL } \\
\mathrm{cm}-\mathrm{s}\end{array}$ & $\begin{array}{c}\text { moist } \\
\%\end{array}$ & $\begin{array}{c}\mathrm{NH4} \\
(\mathrm{CaCL2} 2) \\
\mathrm{mg} / \mathrm{kg}\end{array}$ & $\begin{array}{c}\mathrm{NO3} \\
(\mathrm{CaCL2}) \\
\mathrm{mg} / \mathrm{kg}\end{array}$ & $\begin{array}{l}\mathrm{N} \text { total } \\
\mathrm{mg} / \mathrm{kg}\end{array}$ & $\begin{array}{c}\mathrm{P}) 4 \\
(\mathrm{CaCl} 2) \\
\mathrm{mg} / \mathrm{kg}\end{array}$ & $\begin{array}{l}P \text { total } \\
\mathrm{mg} / \mathrm{kg}\end{array}$ & pH \\
\hline Acer platanoides & 1 & 6.16517 & 2767.62 & 41.29069 & 575.4827 & 65.24673 & 94.82952 & 49.64238 & 59.51403 & 19.62325 & 17.59724 & 8.1157 & 26.43549 & 1722.019 & 2.316761 & 454.3997 & 1.408916 \\
\hline Acorus calamus & 1 & 3.858283 & 5570.313 & 185.666 & & 98.39425 & 193.5949 & 31.25557 & 48.87974 & 28.96824 & 21.82903 & 9.250785 & 23.60263 & 5717.661 & 1.07047 & 572.9593 & 0.913745 \\
\hline Aesculus hippocastanum & 1 & 4.987484 & 2519.466 & 31.89719 & 498.9732 & 54.83706 & 79.83452 & 31.5808 & 38.86641 & 17.14262 & 9.194854 & 9.336315 & 22.6801 & 1354.589 & 2.671061 & 192.3702 & 1.313949 \\
\hline Alnus incana & & 6.685978 & 4370.473 & 69.95927 & 601.6654 & 91.23601 & 175.5315 & 61.87518 & 82.02398 & 54.93049 & 21.34389 & 10.30229 & 33.39916 & 3513.555 & 2.704086 & 304.1132 & 1.494136 \\
\hline Amelanchier lamarckii & 1 & 6.119715 & 2437.315 & 41.39962 & 533.9217 & 61.25719 & 95.39748 & 57.44396 & 75.47767 & 41.56226 & 15.10167 & 8.505918 & 25.03168 & 2583.9 & 2.034907 & 422.6664 & 1.230996 \\
\hline Aronia x prunifolia & 0 & 7.34773 & 5447.726 & 104.9516 & 894.1853 & 72.03701 & 302.7824 & 74.5589 & 105.8139 & 49.85222 & 40.73598 & 16.36107 & 27.21672 & 4328.921 & 2.382297 & 396.8983 & 0.915278 \\
\hline Avena sativa & 0 & 6.806388 & 4800.199 & 88.90757 & 836.4111 & 135.6715 & 139.4913 & 38.49594 & 72.9055 & 40.10229 & 14.69775 & 2.364834 & 14.78412 & 3217.206 & 2.948708 & 718.911 & 1.810661 \\
\hline Bidens frondosa & 1 & 4.91095 & 7769.429 & 236.3176 & 724.4346 & 115.1927 & 191.707 & 51.26082 & 69.24554 & 52.94297 & 29.03287 & 10.55678 & 29.91924 & 7565.567 & 1.623183 & 341.7077 & 1.40968 \\
\hline Castanea sativa & $?$ & 6.753497 & 1819.514 & 27.95912 & 497.5545 & 53.00768 & 80.76171 & 49.16665 & 66.81911 & 31.56929 & 14.40359 & 9.432196 & 33.07851 & 1805.477 & 2.285108 & 710.5315 & 1.095332 \\
\hline Conyza canadensis & 1 & 5.58129 & 4756.722 & 458.5496 & 391.6805 & 147.5272 & 165.0511 & 54.05891 & 65.61157 & 49.91784 & 15.56032 & 2.894039 & 18.88021 & 2951.899 & 1.635343 & 441.5643 & 1.423353 \\
\hline Elodea nuttallii & 1 & 2.677009 & 4491.583 & 205.028 & 252.5928 & 73.90928 & 182.5486 & 20.77773 & 22.2225 & 29.9394 & 16.73872 & 4.955439 & 17.4052 & 4574.238 & 1.108027 & 461.7082 & 0.582324 \\
\hline Epilobium ciliatum & 1 & 5.046353 & 8746.791 & 345.4649 & 755.1176 & 150.1166 & 280.815 & 68.11032 & 92.89639 & 62.07997 & 33.84002 & 10.36546 & 30.69272 & 8343.525 & 1.466342 & 755.3707 & 1.756993 \\
\hline Galinsoga parviflora & 1 & 4.442473 & 4153.86 & 198.6417 & 568.7453 & 127.1169 & 126.5323 & 34.09509 & 45.65105 & 46.5257 & 16.36534 & 2.955358 & 20.45225 & 2925.125 & 2.26559 & 386.572 & 1.288784 \\
\hline Heracleum mantegazzianum & 1 & 6.666722 & 5696.917 & 110.4471 & 802.1123 & 124.3979 & 106.6301 & 45.40697 & 54.96332 & 34.17222 & 15.29858 & 10.41572 & 25.15815 & 3271.714 & 2.313156 & 281.1098 & 2.296013 \\
\hline Impatiens parviflora & 1 & 6.024367 & 3428.021 & 45.54518 & 530.1916 & 72.72944 & 117.3166 & 44.07303 & 60.32589 & 31.07938 & 14.21607 & 10.50337 & 24.0262 & 2340.865 & 2.655233 & 207.1047 & 1.315388 \\
\hline Juncus tenuis & 1 & 7.241008 & 7008.692 & 310.3725 & 610.76 & 130.5202 & 211.2001 & 67.39086 & 93.08524 & 54.25336 & 25.41416 & 9.119558 & 16.90409 & 4966.144 & 1.676167 & 464.8602 & 1.292237 \\
\hline Larix kaempferi & & 5.765885 & 1437.083 & 26.21323 & 510.1595 & 45.02945 & 70.12069 & 47.88359 & 61.81295 & 35.9439 & 12.65798 & 8.854205 & 18.50596 & 1832.52 & 2.190862 & 646.1576 & 0.956368 \\
\hline Oxalis fontana & 1 & 6.101431 & 4269.928 & 82.74247 & 674.1866 & 95.87632 & 102.0917 & 35.76073 & 48.72854 & 43.21306 & 16.95324 & 4.953069 & 17.13375 & 3263.463 & 1.874679 & 327.1302 & 1.522522 \\
\hline Oxycoccus macrocarpos & 0 & 8.463941 & 7937.831 & 225.1449 & 482.3313 & 109.1858 & 288.9503 & 43.49098 & 90.42115 & 33.55568 & 28.04976 & 8.328347 & 10.57364 & 5078.645 & 2.30315 & 395.8604 & 0.979148 \\
\hline Picea abies & & 7.035663 & 1835.427 & 34.53953 & 539.2233 & 60.5169 & 85.7142 & 53.87208 & 69.2537 & 37.06737 & 13.51098 & 10.45761 & 31.87572 & 1989.306 & 2.494504 & 457.4334 & 1.225139 \\
\hline Picea sitchensis & & 7.311648 & 1643.24 & 53.99691 & 508.4278 & 65.29168 & 87.89024 & 50.24077 & 66.3934 & 36.0569 & 9.948059 & 7.188262 & 29.97572 & 2064.336 & 2.140924 & 1003.61 & 1.275102 \\
\hline Pinus nigra & & 8.106574 & 1362.456 & 33.13271 & 1015.675 & 57.90365 & 84.90604 & 44.68616 & 68.62811 & 35.67018 & 10.81378 & 11.45109 & 24.79902 & 2919.148 & 2.561801 & 634.7542 & 1.656316 \\
\hline Pinus nigra v. maritima & & 7.547045 & 1119.416 & 33.24367 & 947.2356 & 56.91661 & 68.44533 & 38.42093 & 53.69981 & 32.82683 & 8.798516 & 6.183718 & 15.159 & 2829.021 & 1.870178 & 1208.587 & 1.218874 \\
\hline Pinus nigra v. nigra & & 10.11812 & 1995.518 & 43.33288 & 1001.988 & 71.75076 & 97.51563 & 50.59494 & 73.91005 & 33.0612 & 12.02952 & 7.554869 & 30.06465 & 2638.09 & 1.745038 & 587.7624 & 2.058725 \\
\hline Pinus sylvestris & & 6.373435 & 1629.255 & 31.11694 & 588.9114 & 52.03704 & 76.61387 & 62.71437 & 78.19636 & 69.55495 & 13.27882 & 9.680099 & 10.598 & 3443.692 & 2.265661 & 657.9857 & 1.014593 \\
\hline Populus gileadensis & & 8.330324 & 4805.206 & 255.2143 & 1013.368 & 127.1656 & 205.3303 & 79.33525 & 102.4773 & 49.36206 & 18.52735 & 10.16046 & 41.2354 & 3883.669 & 2.058782 & 597.9151 & 2.622794 \\
\hline Populus x canadensis & & 5.666064 & 4085.207 & 59.19126 & 538.2198 & 78.637 & 134.0302 & 38.8949 & 48.58161 & 46.78238 & 16.04032 & 7.562232 & 18.51025 & 2872.541 & 1.523557 & 230.4673 & 1.457369 \\
\hline Prunus serotina & 1 & 7.357047 & 2287.531 & 36.4653 & 734.9856 & 61.74162 & 90.92912 & 55.71201 & 73.17603 & 35.0386 & 14.87563 & 10.28842 & 25.08936 & 2641.098 & 2.346862 & 481.218 & 1.353075 \\
\hline Pseudotsuga menziesii & & 5.437465 & 1087.237 & 24.01879 & 463.2996 & 39.65247 & 57.21288 & 39.38763 & 52.50276 & 32.12571 & 10.54108 & 7.402796 & 13.92678 & 1512.492 & 1.888079 & 721.9936 & 0.694001 \\
\hline Quercus rubra & & 7.053439 & 2013.574 & 31.66201 & 609.7072 & 56.7121 & 83.39099 & 55.02065 & 70.45418 & 35.47652 & 14.65067 & 9.739829 & 22.63487 & 1992.29 & 2.066251 & 539.0583 & 1.195784 \\
\hline Robinia pseudoacacia & 1 & 6.461783 & 2708.483 & 33.46638 & 614.7924 & 63.78649 & 91.38255 & 41.50348 & 56.59827 & 27.77456 & 11.31329 & 11.06848 & 31.61228 & 1551.177 & 2.607138 & 285.1863 & 1.558886 \\
\hline Salix daphnoides & 0 & 3.751892 & 7159.699 & 86.29283 & 686.8388 & 134.5127 & 156.5224 & 45.21078 & 62.62001 & 55.56013 & 35.87758 & 6.128463 & 17.19019 & 8251.903 & 1.125676 & 455.3472 & 1.193196 \\
\hline Salix dasyclados & 0 & 3.556005 & 7933.488 & 206.6022 & 581.5359 & 140.6838 & 156.5211 & 64.05931 & 96.2585 & 72.03669 & 31.09122 & 6.33964 & 17.05481 & 6750.593 & 0.86998 & 445.9716 & 1.08668 \\
\hline Secale cereale & 0 & 4.756544 & 4286.822 & 69.68646 & 593.1144 & 129.5946 & 96.86512 & 28.2086 & 39.71584 & 36.99934 & 10.92758 & 2.618074 & 11.21869 & 2185.457 & 2.545371 & 303.1166 & 1.186022 \\
\hline Senecio inaequidens & 1 & 14.00737 & 4172.245 & 80.04053 & 950.8485 & 126.5361 & 133.9952 & 77.76245 & 101.8922 & 45.85653 & 15.47174 & 3.543826 & 21.41408 & 2375.793 & 2.333818 & 562.2936 & 2.511589 \\
\hline Solidago canadensis & $?$ & 7.97786 & 5597.394 & 507.2558 & 939.2526 & 152.0968 & 227.2069 & 48.73286 & 60.72941 & 59.25019 & 37.47932 & 9.12641 & 24.83223 & 7703.137 & 0.794594 & 677.8111 & 2.091087 \\
\hline Solidago gigantea & 1 & 4.560899 & 5037.2 & 368.3431 & 918.5082 & 122.2025 & 262.8192 & 49.14321 & 60.60764 & 40.88319 & 24.83926 & 6.697836 & 22.33475 & 5518.512 & 0.898322 & 328.1889 & 1.266291 \\
\hline Symphoricarpos albus & $?$ & 4.936296 & 2656.648 & 55.2829 & 507.8574 & 57.12624 & 80.32012 & 27.18721 & 34.44041 & 18.11082 & 8.678416 & 5.223833 & 19.16773 & 1555.484 & 1.492629 & 196.856 & 1.16379 \\
\hline Triticum aestivum & 0 & 5.183142 & 6086.612 & 91.06866 & 1027.128 & 174.0774 & 129.1436 & 35.78905 & 42.13859 & 47.0848 & 17.10788 & 3.939036 & 23.02396 & 3975.569 & 1.997283 & 690.8272 & 1.438291 \\
\hline average & & 6.337802 & 4075.183 & 127.4475 & 664.1781 & 93.64696 & 138.7677 & 48.53346 & 65.83432 & 41.127 & 18.32903 & 7.946806 & 22.75891 & 3590.009 & 1.978246 & 501.2405 & 1.391626 \\
\hline s.e. & & 1.978749 & 2153.237 & 127.0482 & 202.0053 & 37.4383 & 66.81562 & 13.91472 & 19.7347 & 12.88022 & 8.436328 & 2.986772 & 6.998684 & 1963.851 & 0.563495 & 216.3783 & 0.446123 \\
\hline
\end{tabular}




\begin{tabular}{|c|c|c|c|c|c|c|c|c|c|c|c|c|c|c|c|c|}
\hline Species name & $\mathrm{C} / \mathrm{N}$ & $\begin{array}{c}\mathrm{Ca}(\mathrm{H} 20) \\
\mathrm{mg} / \mathrm{kg}\end{array}$ & $\begin{array}{c}\mathrm{Cl} \\
\mathrm{mg} / \mathrm{kg}\end{array}$ & $\begin{array}{c}\text { Electronic } \\
\text { conductivity } \\
\mathrm{mS}\end{array}$ & $\begin{array}{c}\mathrm{K} \text { (H20) } \\
\mathrm{mg} / \mathrm{kg}\end{array}$ & $\begin{array}{c}\mathrm{Mg}(\mathrm{H} 20) \\
\mathrm{mg} / \mathrm{kg}\end{array}$ & $\begin{array}{l}\text { MHL } \\
\text { cm -s }\end{array}$ & $\begin{array}{l}\text { MLL } \\
\text { cm -s }\end{array}$ & $\begin{array}{l}\text { MSL } \\
\text { cm -s }\end{array}$ & $\begin{array}{c}\text { moist } \\
\%\end{array}$ & $\begin{array}{c}\text { NH4 } \\
\text { (CaCL2) } \\
\mathrm{mg} / \mathrm{kg} \\
\end{array}$ & $\begin{array}{c}\mathrm{NO3} \\
(\mathrm{CaCL2}) \\
\mathrm{mg} / \mathrm{kg} \\
\end{array}$ & $\begin{array}{l}\mathrm{N} \text { total } \\
\mathrm{mg} / \mathrm{kg}\end{array}$ & $\begin{array}{c}\mathrm{PO4} \\
(\mathrm{CaCl}) \\
\mathrm{mg} / \mathrm{kg} \\
\end{array}$ & $\begin{array}{l}\text { P total } \\
\mathrm{mg} / \mathrm{kg}\end{array}$ & pH \\
\hline Acer campestre & 5.553644 & 3323.07 & 58.2693 & 794.4663 & 66.66616 & 83.23061 & 38.73252 & 38.51169 & 27.278 & 10.63282 & 6.192317 & 20.0749 & 2044.906 & 1.812571 & 293.6717 & 1.709258 \\
\hline Acer pseudoplatanus & 5.939691 & 3052.253 & 42.28228 & 567.2719 & 66.34433 & 100.5146 & 42.83811 & 56.7878 & 25.38807 & 14.41649 & 10.55699 & 25.80448 & 2572.518 & 2.784178 & 440.2174 & 1.454065 \\
\hline Achillea millefolium & 4.526244 & 4726.602 & 71.07194 & 370.232 & 109.482 & 117.145 & 41.61421 & 50.36372 & 31.65867 & 17.15065 & 4.769478 & 14.31548 & 3523.849 & 1.547499 & 398.2702 & 1.279099 \\
\hline Achillea ptarmica & 4.535008 & 7342.648 & 164.8867 & 403.8691 & 121.2373 & 177.1139 & 40.14364 & 44.99661 & 30.82617 & 23.39632 & 6.074322 & 18.32204 & 4510.147 & 1.052815 & 472.1282 & 1.021612 \\
\hline Adoxa moschatellina & 3.287658 & 2701.908 & 41.27066 & 315.654 & 50.54858 & 78.02412 & 38.18353 & 29.93804 & 29.12908 & 12.87618 & 4.183746 & 16.02067 & 2088.851 & 0.862203 & 135.9543 & 0.719121 \\
\hline Aegopodium podagraria & 6.10162 & 3706.445 & 64.09181 & 728.4882 & 73.95944 & 90.23904 & 38.81519 & 43.48735 & 28.43493 & 13.41367 & 6.974243 & 21.71277 & 2396.175 & 1.430624 & 198.4055 & 1.187659 \\
\hline Aethusa cynapium & 3.881247 & 3507.261 & 86.66569 & 745.3573 & 135.5819 & 83.7876 & 32.32471 & 37.20702 & 43.20138 & 11.4013 & 3.919176 & 19.80565 & 2370.34 & 1.546425 & 291.4879 & 0.697776 \\
\hline Agrimonia eupatoria & 3.734833 & 4532.47 & 60.6522 & 521.0123 & 89.9139 & 97.00106 & 39.73775 & 44.27241 & 28.98138 & 15.90561 & 3.709401 & 14.2004 & 3110.67 & 0.665495 & 351.1137 & 0.524096 \\
\hline Agrostis canina & 6.879456 & 9617.248 & 134.1308 & 602.2643 & 136.9248 & 331.0072 & 71.75748 & 104.5859 & 43.18426 & 35.68326 & 11.38887 & 14.28826 & 7660 & 1.929682 & 1049.568 & 1.068945 \\
\hline Agrostis canina ag. (incl. A. vinealis) & 9.181115 & 7836.632 & 81.42574 & 817.6027 & 129.416 & 237.3963 & 123.8988 & 172.7018 & 67.91007 & 42.29991 & 14.49056 & 18.80497 & 808.9232 & 3.492897 & 118.7599 & 1.270761 \\
\hline Agrostis capillaris & 9.846809 & 5700.484 & 69.93728 & 668.8926 & 125.1467 & 163.236 & 72.57885 & 99.92051 & 48.03353 & 26.91459 & 10.7731 & 17.47814 & 4993.225 & 3.233671 & 484.5942 & 1.840516 \\
\hline Agrostis gigantea & 5.917801 & 5988.828 & 192.9489 & 599.5446 & 130.4806 & 238.1821 & 60.41447 & 74.25869 & 54.21917 & 32.07277 & 9.494162 & 26.76384 & 8411.405 & 1.172523 & 638.5303 & 1.432781 \\
\hline Agrostis stolonifera & 7.373595 & 8654.556 & 685.0757 & 513.2532 & 137.3677 & 269.8903 & 59.72882 & 79.80272 & 55.3702 & 32.91927 & 8.028078 & 19.88874 & 9381.599 & 1.573761 & 907.8245 & 1.995342 \\
\hline Agrostis vinealis & 8.654843 & 1971.134 & 30.76981 & 905.8192 & 53.42281 & 51.48783 & 64.17541 & 81.69516 & 72.88498 & 10.01233 & 7.565694 & 7.061162 & 2228.212 & 1.018793 & 399.0688 & 1.297753 \\
\hline Aira caryophyllea & 4.791975 & 3285.402 & 27.30597 & 211.3393 & 84.4749 & 104.3367 & 47.18574 & 53.86791 & 31.88306 & 13.8188 & 3.309402 & 9.585471 & 2144.801 & 1.231763 & 274.8699 & 0.998236 \\
\hline Aira praecox & 6.779396 & 2250.058 & 32.8437 & 193.8455 & 49.83318 & 81.75433 & 57.17707 & 58.02215 & 31.43125 & 11.82145 & 3.903406 & 11.08733 & 1782.29 & 1.473976 & 287.4814 & 1.824481 \\
\hline Ajuga reptans & 6.416167 & 9218.204 & 57.3348 & 694.935 & 96.31054 & 174.6696 & 66.38559 & 75.44723 & 50.28471 & 31.46442 & 6.389014 & 28.83635 & 6358.606 & 1.680647 & 494.019 & 1.199106 \\
\hline Alisma lanceolatum & 2.965506 & 4143.867 & 191.8205 & 330.8307 & 64.31442 & 214.816 & 31.84343 & 44.79371 & 37.20504 & 21.74558 & 5.978473 & 15.60224 & 5772.576 & 0.885605 & 344.8429 & 0.778562 \\
\hline Alisma plantago-aquatica & 4.035376 & 5504.222 & 204.3954 & 409.4099 & 98.19089 & 201.3708 & 32.99527 & 44.88435 & 37.30851 & 20.8982 & 10.93516 & 28.40001 & 5230.332 & 1.514224 & 525.6195 & 0.950735 \\
\hline Alliaria petiolata & 4.935723 & 3075.666 & 60.75243 & 661.6413 & 66.25094 & 79.82954 & 33.93016 & 34.34582 & 20.28321 & 11.37803 & 5.312887 & 15.99306 & 2381.434 & 1.064361 & 365.5297 & 1.271545 \\
\hline Allium oleraceum & 5.922544 & 3785.79 & 48.0961 & 371.4105 & 78.04263 & 94.2711 & 38.99342 & 42.37737 & 40.98052 & 14.14921 & 5.226552 & 28.86691 & 3096.785 & 1.081228 & 461.0544 & 1.003792 \\
\hline Allium scorodoprasum & 7.76479 & 2470.813 & 33.31511 & 1058.45 & 48.43974 & 69.30454 & 27.73533 & 24.42522 & 17.23954 & 10.64836 & 4.377808 & 16.53118 & 2432.562 & 0.872364 & 353.6148 & 1.330504 \\
\hline Allium ursinum & 3.454485 & 2484.223 & 28.71658 & 639.9525 & 48.37697 & 64.3204 & 26.94602 & 19.93194 & 22.23464 & 10.04266 & 5.158146 & 15.06589 & 1061.293 & 0.889253 & 193.0752 & 0.801023 \\
\hline Allium vineale & 6.737198 & 4815.062 & 68.65175 & 613.4722 & 107.855 & 120.2328 & 39.53333 & 44.93058 & 38.1779 & 16.36824 & 5.426008 & 27.4985 & 3235.747 & 1.179598 & 387.8566 & 1.061935 \\
\hline Alnus glutinosa & 5.637878 & 5896.373 & 135.1159 & 561.7079 & 89.23107 & 314.2781 & 72.66927 & 91.96947 & 62.85787 & 22.51251 & 10.02754 & 24.80724 & 4075.25 & 1.969467 & 504.1171 & 1.144964 \\
\hline Alopecurus aequalis & 4.597707 & 6996.266 & 220.5729 & 446.1218 & 123.4497 & 190.4749 & 36.25392 & 50.533 & 38.71231 & 27.53085 & 6.718945 & 22.12153 & 7553.293 & 1.191358 & 628.3343 & 1.161479 \\
\hline Alopecurus bulbosus & 2.178242 & 4317.051 & 608.6053 & 449.0124 & 68.12205 & 118.8016 & 23.1107 & 26.64721 & 29.48927 & 17.78423 & 4.317767 & 9.147256 & 2954.449 & 0.798268 & 291.6229 & 0.933589 \\
\hline Alopecurus geniculatus & 2.734556 & 5213.758 & 440.9543 & 332.7738 & 87.2176 & 141.1182 & 31.65024 & 49.08594 & 37.12234 & 23.59648 & 4.156207 & 9.149609 & 7017.149 & 0.691765 & 377.2957 & 0.721991 \\
\hline Alopecurus myosuroides & 2.348498 & 3113.243 & 82.69111 & 751.5198 & 99.64728 & 83.20326 & 22.87734 & 24.80097 & 40.56239 & 10.95328 & 3.281431 & 10.62846 & 2567.115 & 1.258233 & 241.3249 & 0.475475 \\
\hline Alopecurus pratensis & 5.312341 & 7110.489 & 91.29173 & 510.1124 & 113.2155 & 96.07852 & 35.2163 & 44.83377 & 48.04288 & 24.36045 & 4.598115 & 16.64041 & 3753.711 & 0.76054 & 303.9979 & 0.664844 \\
\hline Althaea officinalis & 3.656425 & 5553.65 & 596.1748 & 752.2998 & 98.31757 & 157.8961 & 33.37147 & 39.94334 & 32.74152 & 17.53929 & 6.146283 & 17.39199 & 3993.133 & 0.978752 & 264.1862 & 0.937889 \\
\hline Ammophila arenaria & 4.032584 & 2268.641 & 527.5923 & 212.5634 & 59.41648 & 99.43565 & 60.19599 & 68.03287 & 31.33833 & 11.30267 & 4.509116 & 13.30597 & 1699.967 & 1.42225 & 292.9166 & 1.84412 \\
\hline Anagallis arvensis & 2.525799 & 4503.157 & 108.9576 & 677.9739 & 155.9315 & 114.0621 & 38.11684 & 42.58138 & 42.44886 & 12.89345 & 3.337189 & 15.5589 & 2751.919 & 2.025583 & 531.078 & 0.745244 \\
\hline Anagallis arvensis s. arvensis & 3.492352 & 4604.372 & 638.2115 & 552.2331 & 163.8482 & 106.6016 & 35.51624 & 39.48048 & 39.89845 & 12.25704 & 3.341238 & 17.1612 & 2693.75 & 2.293342 & 560.3401 & 0.931211 \\
\hline Anagallis minima & 3.318969 & 7159.185 & 629.2507 & 301.8621 & 108.1912 & 267.7993 & 47.22908 & 53.67138 & 41.58045 & 15.52838 & 4.484567 & 9.976542 & 3019.399 & 0.673771 & 256.2658 & 1.480186 \\
\hline Anagallis tenella & 3.384728 & 5758.012 & 436.6782 & 405.1556 & 95.00838 & 120.6325 & 33.91641 & 52.38126 & 31.68867 & 12.09563 & 3.736515 & 12.00232 & 3171.43 & 0.974765 & 278.3446 & 1.18864 \\
\hline Anchusa arvensis & 4.578853 & 3721.596 & 92.94245 & 371.64 & 112.6595 & 133.1816 & 55.39737 & 57.37906 & 37.13969 & 12.43323 & 2.626966 & 17.92757 & 2442.281 & 2.798679 & 515.7861 & 1.306846 \\
\hline Anchusa officinalis & 6.102222 & 3406.308 & 95.59757 & 386.4145 & 107.4476 & 107.9167 & 45.31918 & 47.96818 & 35.31742 & 12.40084 & 4.652818 & 32.24073 & 2210.329 & 1.571195 & 408.6552 & 1.214869 \\
\hline Andromeda polifolia & 3.710049 & 2653.32 & 20.04692 & 828.2824 & 62.54759 & 173.5735 & 95.93347 & 147.0632 & 35.13238 & 18.77482 & 9.771154 & 5.721451 & 3962.644 & 2.034016 & 360.6986 & 0.458701 \\
\hline Anemone nemorosa & 5.427614 & 5818.883 & 41.24923 & 560.7899 & 66.07784 & 152.2514 & 65.82086 & 75.16424 & 57.23234 & 25.65216 & 7.65494 & 29.98319 & 3897.738 & 2.715884 & 171.6488 & 0.993506 \\
\hline Angelica archangelica & 3.152154 & 6691.121 & 223.4709 & 678.0312 & 128.3957 & 179.4072 & 44.69194 & 58.89403 & 41.38351 & 26.23683 & 5.306177 & 18.26666 & 4814.57 & 0.747151 & 290.3407 & 0.879882 \\
\hline Angelica sylvestris & 5.291821 & 7883.712 & 182.6264 & 534.2982 & 87.57811 & 220.4719 & 56.03874 & 67.21058 & 45.64413 & 24.0398 & 7.478378 & 29.28092 & 4332.42 & 1.481196 & 344.0576 & 0.966121 \\
\hline Anisantha sterilis & 6.16016 & 4172.38 & 71.44652 & 720.3605 & 114.2764 & 129.2138 & 38.20658 & 42.83907 & 31.04505 & 14.88365 & 5.878862 & 27.6088 & 3412.032 & 1.85566 & 541.1221 & 1.056561 \\
\hline Anisantha tectorum & 1.459299 & 3304.303 & 63.56183 & 173.0346 & 91.09811 & 121.0816 & 65.86107 & 56.31977 & 34.19043 & 13.72784 & 2.787053 & 21.11183 & 2028.097 & 1.984129 & 351.6737 & 0.572769 \\
\hline
\end{tabular}




\begin{tabular}{|c|c|c|c|c|c|c|c|c|c|c|c|c|c|c|c|c|}
\hline Antennaria dioica & 7.351993 & 2302.798 & 20.57806 & 397.9462 & 52.67561 & 85.46759 & 52.8037 & 62.2562 & 39.11485 & 12.69626 & 5.090902 & 7.794092 & 2303.381 & 0.54036 & 284.2976 & 1.432304 \\
\hline Anthemis arvensis & 3.544868 & 4457.437 & 69.07657 & 474.1378 & 133.7456 & 113.8831 & 31.23274 & 41.71442 & 36.26916 & 11.53527 & 2.418857 & 11.04196 & 2390.133 & 2.701015 & 421.9078 & 0.968335 \\
\hline Anthoxanthum aristatum & 6.261831 & 4016.487 & 73.94596 & 578.6478 & 105.4251 & 112.0004 & 42.18348 & 64.74885 & 48.86008 & 12.52291 & 2.585819 & 13.30047 & 2853.676 & 2.753179 & 478.9505 & 1.221849 \\
\hline Anthoxanthum odoratum & 7.811885 & 9094.018 & 140.7187 & 451.9596 & 127.4241 & 218.9278 & 65.14042 & 85.68595 & 38.49262 & 33.16262 & 7.763766 & 12.71575 & 6976.229 & 2.124331 & 553.9533 & 1.383222 \\
\hline Anthriscus caucalis & 5.43778 & 2559.168 & 61.2544 & 498.9656 & 87.42629 & 98.18323 & 37.85693 & 38.29556 & 33.89342 & 10.81608 & 7.127421 & 27.93848 & 2002.233 & 1.163075 & 383.8071 & 0.700856 \\
\hline Anthriscus sylvestris & 6.917802 & 5028.874 & 103.2585 & 848.1067 & 97.6112 & 98.86631 & 39.20676 & 46.13021 & 39.17866 & 17.11264 & 6.09453 & 26.15285 & 3201.466 & 1.434572 & 289.5 & 1.31075 \\
\hline Anthyllis vulneraria & 1.471314 & 3305.387 & 406.7443 & 158.7558 & 82.19233 & 116.5951 & 62.55136 & 52.87829 & 37.54327 & 15.19604 & 3.438752 & 10.70903 & 1903.256 & 0.461212 & 305.4064 & 1.37443 \\
\hline Apera spica-venti & 3.575989 & 3792.209 & 78.07875 & 542.2507 & 115.6397 & 99.85463 & 30.38285 & 39.95347 & 41.72569 & 10.74224 & 2.880683 & 11.33641 & 2457.404 & 2.642387 & 403.3472 & 0.967747 \\
\hline Aphanes arvensis & 3.222416 & 4018.928 & 73.65564 & 557.1351 & 112.366 & 101.9412 & 30.08751 & 39.55112 & 41.44332 & 11.57936 & 2.952679 & 10.35261 & 2303.67 & 2.654418 & 339.406 & 0.876961 \\
\hline Aphanes inexpectata & 4.830071 & 4234.952 & 52.49006 & 351.2151 & 114.0467 & 111.0087 & 45.95206 & 115.0478 & 53.39401 & 13.60524 & 2.62248 & 12.46144 & 2611.098 & 3.326453 & 398.0567 & 1.116251 \\
\hline Apium graveolens & 3.600883 & 7143.805 & 627.7103 & 527.8038 & 104.5651 & 242.0339 & 36.08179 & 39.94159 & 41.49481 & 16.97301 & 6.008547 & 19.16928 & 6816.045 & 1.438157 & 599.5019 & 0.805333 \\
\hline Apium nodiflorum & 4.629503 & 7645.097 & 411.1023 & 757.2368 & 118.5684 & 206.0534 & 53.72538 & 66.47574 & 54.98307 & 27.0229 & 6.257507 & 24.55266 & 6725.265 & 1.301643 & 580.3969 & 0.839749 \\
\hline Arabidopsis thaliana & 4.768638 & 4633.849 & 63.63401 & 361.5492 & 115.7328 & 135.4405 & 65.08461 & 57.85686 & 39.60557 & 16.36259 & 3.237977 & 13.44873 & 3073.201 & 3.195931 & 508.2633 & 1.199171 \\
\hline Arabis glabra & 6.569807 & 2897.392 & 48.09245 & 328.9772 & 99.05438 & 115.792 & 56.87701 & 77.10113 & 41.06219 & 16.40355 & 6.055838 & 24.94991 & 2424.282 & 1.68461 & 392.1011 & 1.226755 \\
\hline Arabis hirsuta & 2.634735 & 2279.851 & 38.7585 & 243.0731 & 49.1406 & 59.78147 & 40.78498 & 41.95183 & 35.98285 & 11.33493 & 6.247325 & 13.38103 & 1457.082 & 1.123295 & 225.9012 & 0.718046 \\
\hline Arabis hirsuta s. hirsuta & 5.730017 & 2952.143 & 42.53969 & 269.943 & 83.6239 & 92.53001 & 57.7858 & 66.58061 & 32.77908 & 12.28486 & 4.539864 & 12.29122 & 1617.088 & 1.785788 & 257.7567 & 0.970443 \\
\hline Arctium lappa & 5.686904 & 4431.915 & 105.2655 & 897.0077 & 107.6679 & 128.5858 & 39.63551 & 46.15611 & 41.71276 & 18.95033 & 6.989995 & 23.25756 & 3617.676 & 1.025706 & 401.9987 & 1.335952 \\
\hline Arctium minus (excl. A. pubens) & 7.085031 & 4183.32 & 76.53232 & 649.1573 & 119.5085 & 87.25432 & 38.75205 & 45.19363 & 35.52591 & 12.33407 & 6.04402 & 23.11949 & 3065.553 & 1.078629 & 467.5917 & 1.233932 \\
\hline Arctium pubens & 6.432861 & 3565.993 & 131.7203 & 934.9807 & 108.7089 & 101.664 & 38.52304 & 43.70124 & 33.43934 & 12.9707 & 6.987739 & 23.01253 & 3029.523 & 0.887563 & 504.5692 & 1.312827 \\
\hline Arenaria serpyllifolia & 1.938179 & 4261.598 & 264.0293 & 199.0447 & 114.214 & 129.922 & 64.69401 & 57.26402 & 29.45815 & 14.42314 & 3.434176 & 11.80148 & 2221.18 & 1.565831 & 363.1853 & 0.710249 \\
\hline Armeria maritima & 1.990896 & 7954.478 & 583.1083 & 228.3172 & 185.9555 & 249.8925 & 46.40932 & 52.67368 & 31.07785 & 30.2288 & 4.468799 & 13.16717 & 2716.63 & 0.577278 & 385.4498 & 0.887281 \\
\hline Arnica montana & 5.788717 & 2726.631 & 14.5475 & 818.6378 & 53.3281 & 100.68 & 59.13965 & 90.34479 & 29.04597 & 16.07503 & 4.314517 & 5.581344 & 3163.29 & 0.651358 & 259.4707 & 0.602473 \\
\hline Arnoseris minima & 2.965136 & 3545.042 & 72.7796 & 518.487 & 89.73277 & 92.18603 & 30.52101 & 43.43239 & 44.55757 & 9.609985 & 2.77194 & 8.368597 & 2491.03 & 2.397483 & 284.031 & 0.672996 \\
\hline Arrhenatherum elatius & 6.168792 & 5300.969 & 67.36348 & 606.1643 & 98.92802 & 100.0029 & 37.22202 & 45.70093 & 48.61617 & 16.02817 & 5.154788 & 17.67635 & 3363.752 & 1.349317 & 334.0468 & 1.206413 \\
\hline Arrhenatherum elatius s. elatius & 4.662947 & 3677.523 & 76.05932 & 573.8088 & 91.80158 & 88.9086 & 28.78676 & 34.59832 & 45.32042 & 12.75918 & 4.85749 & 18.70472 & 2949.827 & 1.428609 & 300.4877 & 0.781071 \\
\hline Artemisia campestris s. campestris & 1.677949 & 2553.406 & 29.14943 & 130.193 & 64.87223 & 73.72953 & 31.59768 & 39.49602 & 22.8903 & 8.907786 & 3.868582 & 12.26081 & 1435.53 & 1.274897 & 247.6502 & 1.176951 \\
\hline Artemisia campestris s. maritima & 1.083691 & 2991.326 & 797.8153 & 128.0212 & 71.18491 & 101.0923 & 68.30115 & 55.66708 & 28.9741 & 15.56056 & 2.898366 & 11.09469 & 1860.84 & 2.065603 & 315.8609 & 0.633219 \\
\hline Artemisia vulgaris & 5.963758 & 3770.168 & 171.8257 & 616.0678 & 113.6484 & 113.5137 & 37.38379 & 45.41746 & 46.23368 & 14.67581 & 6.109192 & 23.47951 & 3188.334 & 1.754297 & 440.1564 & 1.222802 \\
\hline Arum maculatum & 3.503359 & 2270.191 & 31.12444 & 469.6786 & 45.39761 & 55.90643 & 31.20988 & 23.65408 & 23.68389 & 9.337674 & 4.040109 & 14.69685 & 1212.403 & 0.827446 & 134.6438 & 0.77628 \\
\hline Asparagus officinalis & 5.18164 & 3789.951 & 488.9529 & 500.2494 & 99.03109 & 214.9835 & 80.83609 & 85.83608 & 67.70228 & 19.07457 & 7.883896 & 29.52333 & 2551.767 & 1.490062 & 338.6893 & 1.8077 \\
\hline Asparagus officinalis s. officinalis & 5.75766 & 2771.057 & 61.46205 & 389.8216 & 81.34731 & 95.33047 & 44.17633 & 46.76849 & 36.15005 & 12.86482 & 6.158631 & 23.02838 & 1858.24 & 1.27542 & 282.5668 & 1.138549 \\
\hline Asparagus officinalis s. prostratus & 1.272641 & 2749.239 & 37.17723 & 167.0979 & 67.82952 & 104.694 & 51.54708 & 43.11077 & 29.04778 & 14.42242 & 4.482425 & 10.02432 & 1816.37 & 1.773498 & 288.9447 & 0.728597 \\
\hline Aster tripolium & 2.991751 & 6989.41 & 590.4743 & 690.559 & 112.5547 & 228.7521 & 28.91261 & 30.53727 & 45.09732 & 20.57311 & 6.642938 & 22.23854 & 3785.729 & 1.647504 & 367.8843 & 0.822976 \\
\hline Athyrium filix-femina & 4.735604 & 3711.889 & 59.26832 & 323.3541 & 70.13533 & 147.339 & 47.90556 & 60.45724 & 40.15899 & 18.10657 & 7.709 & 21.07448 & 2884.47 & 1.51674 & 233.7084 & 1.01988 \\
\hline Atriplex littoralis & 1.959782 & 7775.087 & 671.7371 & 487.4805 & 152.6172 & 290.7664 & 30.99729 & 35.85348 & 39.0149 & 21.10922 & 4.248836 & 27.77593 & 3153.79 & 1.650032 & 526.4811 & 0.876241 \\
\hline Atriplex patula & 5.024056 & 4477.717 & 580.0375 & 617.6802 & 132.666 & 119.5581 & 37.33779 & 47.51263 & 52.99623 & 18.21138 & 5.007755 & 16.61366 & 4292.317 & 1.598998 & 523.6766 & 1.296611 \\
\hline Atriplex prostrata & 4.37594 & 6720.447 & 691.7886 & 848.0858 & 134.8614 & 179.3497 & 40.64212 & 48.46856 & 58.63921 & 27.89041 & 7.46417 & 24.77281 & 5598.246 & 1.53275 & 622.8964 & 0.997809 \\
\hline Avena fatua & 4.459046 & 3440.392 & 298.1313 & 767.1499 & 126.1406 & 96.88726 & 30.34212 & 37.80876 & 49.63031 & 10.34865 & 3.047817 & 13.1871 & 2542.409 & 2.889716 & 307.3122 & 1.115347 \\
\hline Ballota nigra & 6.011378 & 2445 & 62.6148 & 781.2641 & 74.75321 & 72.69144 & 26.39806 & 30.36376 & 20.92568 & 9.021173 & 4.564978 & 24.10186 & 2550.037 & 1.127905 & 544.5954 & 1.192784 \\
\hline Ballota nigra s. foetida & 6.942911 & 3125.68 & 150.7724 & 631.9996 & 126.9707 & 76.98683 & 35.17886 & 39.53655 & 25.55281 & 11.63045 & 6.720943 & 27.3332 & 2308.65 & 1.003517 & 631.5308 & 1.337266 \\
\hline Barbarea stricta & 4.076517 & 7766.225 & 132.7145 & 882.478 & 128.089 & 298.8809 & 69.39081 & 92.20175 & 65.67731 & 35.58728 & 11.57268 & 24.86925 & 9162.625 & 1.604271 & 626.6085 & 0.943078 \\
\hline Barbarea vulgaris & 7.542144 & 7398.622 & 409.0989 & 886.0582 & 109.9605 & 172.7329 & 51.97546 & 65.9588 & 66.38892 & 36.25841 & 10.13587 & 31.40094 & 6617.428 & 2.06861 & 844.315 & 1.058326 \\
\hline Bellis perennis & 2.153817 & 5706.261 & 430.357 & 455.2241 & 108.985 & 100.7773 & 29.60499 & 35.82925 & 24.83869 & 16.72687 & 3.755223 & 9.275012 & 4121.982 & 0.730607 & 418.0048 & 0.667419 \\
\hline Berberis vulgaris & 5.471725 & 2397.876 & 45.75517 & 653.0833 & 60.17998 & 82.89375 & 33.57701 & 35.32123 & 31.10542 & 11.68204 & 5.432372 & 28.92366 & 1997.091 & 1.991344 & 306.4007 & 1.952016 \\
\hline Berteroa incana & 4.024229 & 7050.361 & 68.18574 & 294.1144 & 105.7382 & 112.1241 & 39.98373 & 56.35787 & 53.47687 & 35.5446 & 3.00154 & 11.54229 & 5576.676 & 2.137313 & 356.3272 & 1.324257 \\
\hline Berula erecta & 3.921223 & 4955.962 & 232.5751 & 512.7483 & 94.78428 & 187.6363 & 31.228 & 45.08026 & 31.53052 & 22.32929 & 9.755419 & 27.07465 & 77.936 & 1.085107 & 69.4375 & .891057 \\
\hline
\end{tabular}




\begin{tabular}{|c|c|c|c|c|c|c|c|c|c|c|c|c|c|c|c|c|}
\hline Betula pendula & 8.065446 & 2549.655 & 57.08344 & 639.4356 & 68.00721 & 101.0573 & 64.49637 & 83.83722 & 49.75176 & 15.65661 & 10.69583 & 25.08363 & 2710.869 & 2.493587 & 429.8334 & 1.382535 \\
\hline Betula pendula + B. pubescens & 7.469253 & 3446.497 & 111.3419 & 613.7033 & 79.18442 & 141.4198 & 72.56579 & 89.74038 & 70.02363 & 13.61205 & 7.431521 & 26.50254 & 2754.884 & 2.785364 & 348.0981 & 2.02266 \\
\hline Betula pubescens & 8.123633 & 3984.391 & 82.44584 & 669.9323 & 80.78943 & 220.469 & 88.98263 & 124.5713 & 59.43152 & 23.77947 & 10.12633 & 29.05384 & 4531.372 & 2.504123 & 565.232 & 1.339356 \\
\hline Bidens cernua & 4.909961 & 6646.934 & 239.9132 & 445.4067 & 97.69237 & 209.3864 & 27.83775 & 38.11561 & 32.44312 & 23.59808 & 8.62873 & 27.92598 & 5808.711 & 1.244754 & 521.2032 & 1.413503 \\
\hline Bidens connata & 4.903588 & 6769.764 & 247.699 & 523.1918 & 100.219 & 210.2026 & 37.46872 & 52.4433 & 39.71986 & 24.49019 & 7.999397 & 28.88317 & 6523.565 & 1.04629 & 590.0761 & 1.101391 \\
\hline Bidens tripartita & 4.893432 & 8122.554 & 364.548 & 543.1603 & 135.3958 & 264.3523 & 52.0935 & 71.30836 & 52.35249 & 32.3214 & 8.56106 & 27.22674 & 7735.516 & 1.770725 & 502.2361 & 1.48905 \\
\hline Blackstonia perfoliata & 2.270902 & 7518.462 & 542.0222 & 349.7105 & 84.11801 & 234.8032 & 34.32444 & 35.7806 & 41.4194 & 15.01697 & 5.235838 & 12.71834 & 3886.391 & 0.774042 & 241.4187 & 0.968127 \\
\hline Blechnum spicant & 5.923841 & 2434.219 & 39.27601 & 599.7043 & 54.18333 & 79.20842 & 55.93702 & 63.27959 & 45.74744 & 11.77391 & 9.076017 & 20.29973 & 1996.581 & 2.37855 & 399.036 & 1.144191 \\
\hline Blysmus compressus & 2.205941 & 4863.91 & 550.196 & 377.3149 & 102.4592 & 130.2754 & 28.24198 & 24.1018 & 33.40647 & 9.858232 & 3.797471 & 7.463809 & 3171.057 & 0.683848 & 259.2465 & 1.029539 \\
\hline Bolboschoenus maritimus & 4.632602 & 6473.107 & 612.7749 & 558.4623 & 87.17716 & 185.5524 & 43.02972 & 50.70151 & 43.66441 & 28.33355 & 7.284235 & 26.55229 & 7998.864 & 1.183469 & 667.6459 & 1.091242 \\
\hline Botrychium lunaria & 6.454502 & 2876.083 & 36.42556 & 234.7209 & 67.60035 & 89.29671 & 56.32005 & 52.94716 & 38.76952 & 14.98199 & 4.976253 & 11.84044 & 1700.804 & 0.83246 & 222.6605 & 1.841325 \\
\hline Brachypodium pinnatum & 1.473399 & 3278.536 & 29.90269 & 454.8972 & 70.26512 & 93.00102 & 41.02539 & 37.10311 & 45.01094 & 8.976089 & 3.65655 & 18.11525 & 2070.787 & 0.496397 & 286.2895 & 0.523899 \\
\hline Brachypodium sylvaticum & 4.490714 & 2716.586 & 37.60204 & 668.9325 & 47.17941 & 68.8878 & 35.37836 & 28.60818 & 30.2557 & 9.196525 & 5.24672 & 16.799 & 1502.824 & 1.679856 & 160.2837 & 1.41841 \\
\hline Brassica napus & 5.20698 & 5049.446 & 93.04177 & 889.7947 & 110.4131 & 125.8455 & 38.17727 & 48.75633 & 50.47833 & 32.82042 & 5.987314 & 24.65295 & 5500.824 & 2.880033 & 339.5658 & 1.203559 \\
\hline Brassica nigra & 4.89283 & 3742.623 & 183.4604 & 741.0835 & 107.3435 & 99.14015 & 40.64295 & 50.21082 & 56.09445 & 19.93123 & 6.599761 & 25.24037 & 4416.675 & 1.288171 & 415.3854 & 0.812194 \\
\hline Briza media & 3.850899 & 5688.486 & 60.86742 & 342.684 & 75.57408 & 166.9478 & 60.97069 & 65.45428 & 35.65588 & 31.40005 & 5.099984 & 10.30544 & 6774.578 & 0.591834 & 283.6968 & 1.367952 \\
\hline Bromopsis erecta & 1.410618 & 4146.873 & 63.64121 & 391.2757 & 135.815 & 93.26099 & 72.59168 & 72.8692 & 36.85638 & 10.5053 & 4.037082 & 9.232763 & 3954.857 & 0.497815 & 318.852 & 0.625493 \\
\hline Bromopsis inermis & 1.916509 & 3940.415 & 100.3757 & 426.4089 & 121.3292 & 78.9854 & 33.66868 & 37.60089 & 42.57812 & 14.09942 & 4.033825 & 17.99382 & 3167.416 & 1.261999 & 317.8794 & 0.47328 \\
\hline Bromus hordeaceus & 2.567526 & 9154.073 & 469.5632 & 429.1378 & 190.7224 & 155.8583 & 62.73286 & 67.58658 & 40.23734 & 24.3104 & 4.933195 & 10.99491 & 5709.896 & 1.152688 & 617.3461 & 0.985351 \\
\hline Bromus hordeaceus s. hordeaceus & 3.194977 & 7276.572 & 117.115 & 444.124 & 173.1357 & 171.0562 & 53.78975 & 60.47721 & 30.43173 & 21.37061 & 5.148915 & 14.72908 & 5218.492 & 1.184691 & 582.0282 & 0.945672 \\
\hline Bromus hordeaceus s. thominei & 1.231408 & 2230.077 & 31.75428 & 90.069 & 62.98225 & 88.73728 & 62.64702 & 48.92474 & 36.6589 & 10.15922 & 3.268834 & 7.513131 & 1621.091 & 1.582056 & 272.3333 & 1.168869 \\
\hline Bromus racemosus & 3.313274 & 5102.77 & 118.2213 & 410.7751 & 97.38088 & 112.9403 & 29.01913 & 31.72466 & 47.5103 & 21.13727 & 4.434541 & 24.04781 & 5140.793 & 0.483003 & 477.0409 & 0.508009 \\
\hline Bromus racemosus s. racemosus & 2.083285 & 5397.525 & 503.8881 & 359.0727 & 96.18027 & 91.9497 & 26.63362 & 31.74859 & 25.04795 & 23.62682 & 2.87213 & 7.379419 & 5186.285 & 0.853192 & 409.4413 & 0.627152 \\
\hline Bryonia dioica & 5.19833 & 2712.927 & 61.93622 & 762.7862 & 73.24686 & 91.14265 & 35.06221 & 39.6548 & 30.51484 & 12.54781 & 5.775227 & 24.98696 & 2562.386 & 0.894496 & 407.5128 & 1.364293 \\
\hline Butomus umbellatus & 2.918605 & 4804.055 & 209.1297 & 326.9871 & 71.17932 & 171.5044 & 19.83956 & 24.68806 & 25.15016 & 18.61872 & 6.040817 & 25.2735 & 4750.427 & 0.998956 & 435.3677 & 0.60382 \\
\hline Calamagrostis canescens & 5.843936 & 8891.084 & 118.3347 & 495.8608 & 111.408 & 297.7504 & 72.12063 & 100.1605 & 48.40465 & 31.37378 & 9.384631 & 28.61297 & 5115.902 & 1.94619 & 745.7781 & 1.032767 \\
\hline Calamagrostis epigejos & 7.736517 & 4460.611 & 510.2066 & 460.5919 & 114.4959 & 228.1687 & 92.62593 & 92.95845 & 72.66028 & 19.45103 & 7.299816 & 25.30653 & 2973.28 & 1.651675 & 386.0901 & 1.920254 \\
\hline Calamagrostis stricta & 3.083923 & 4876.757 & 96.92398 & 310.9347 & 126.1009 & 154.4432 & 21.61581 & 33.14666 & 16.25796 & 16.92587 & 5.775405 & 11.08027 & 3007.911 & 1.165774 & 623.3049 & 0.499746 \\
\hline Calammophila baltica & 1.782324 & 2502.416 & 746.9534 & 233.4964 & 82.85709 & 113.4177 & 59.83348 & 54.59245 & 80.7648 & 10.59987 & 6.060972 & 24.43252 & 1654.288 & 1.575681 & 302.4945 & 0.693976 \\
\hline Calla palustris & 6.140797 & 7833.398 & 253.0464 & 492.7202 & 137.3333 & 191.7684 & 45.73958 & 66.88717 & 36.41889 & 25.42359 & 9.877346 & 21.94586 & 6185.798 & 1.755064 & 583.595 & 1.659142 \\
\hline Callitriche obtusangula & 2.576429 & 5871.248 & 276.2012 & 353.2639 & 98.50083 & 198.6017 & 30.16812 & 41.37767 & 35.33381 & 17.46617 & 4.87428 & 18.4027 & 4610.013 & 1.140911 & 499.4515 & 0.791732 \\
\hline Callitriche platycarpa & 5.987208 & 7897.308 & 283.5109 & 405.1569 & 135.7921 & 220.746 & 39.38986 & 50.90038 & 40.56305 & 20.53979 & 8.073934 & 24.04247 & 5352.622 & 1.602145 & 479.2381 & 1.189619 \\
\hline Callitriche stagnalis & 4.740211 & 8267.314 & 365.7433 & 717.8272 & 130.8608 & 251.7528 & 44.79158 & 60.59897 & 44.73139 & 24.68669 & 7.425876 & 24.59848 & 6490.237 & 1.526667 & 489.583 & 1.610045 \\
\hline Calluna vulgaris & 8.885939 & 2012.993 & 23.42489 & 1106.002 & 63.63132 & 92.47385 & 75.81665 & 95.73338 & 50.10261 & 15.90058 & 10.38143 & 7.33103 & 3197.32 & 2.173929 & 457.4038 & 1.228182 \\
\hline Caltha palustris & 4.664584 & 5305.25 & 172.1184 & 507.9216 & 110.2764 & 212.3269 & 42.08521 & 56.72667 & 33.86994 & 19.7542 & 8.935885 & 29.88282 & 4912.742 & 1.207913 & 665.6212 & 0.683091 \\
\hline Caltha palustris s. araneosa & 2.605539 & 7573.415 & 235.2308 & 651.223 & 103.101 & 152.6846 & 49.45267 & 63.16196 & 40.29945 & 23.09523 & 5.473145 & 18.64822 & 5335.368 & 1.633878 & 256.2586 & 0.445689 \\
\hline Caltha palustris s. palustris & 5.549273 & 7231.082 & 176.6682 & 508.6812 & 123.0899 & 218.6025 & 51.75563 & 71.92024 & 38.1028 & 24.77984 & 8.171783 & 26.57618 & 6325.213 & 1.08275 & 631.7317 & 0.866275 \\
\hline Calystegia sepium & 5.003482 & 7470.553 & 280.7587 & 688.1358 & 110.2391 & 228.2315 & 57.61742 & 68.53785 & 71.24046 & 28.78283 & 10.52874 & 23.07547 & 5234.97 & 1.619543 & 421.3833 & 1.511862 \\
\hline Campanula rapunculoides & 4.710623 & 3728.263 & 109.4846 & 419.2942 & 145.2857 & 80.25973 & 50.40635 & 48.25568 & 40.4761 & 13.0507 & 4.556555 & 21.59207 & 2254.26 & 2.001237 & 381.997 & 1.20782 \\
\hline Campanula rapunculus & 2.847894 & 3663.252 & 49.64211 & 323.5687 & 70.09315 & 76.71916 & 29.02731 & 34.47939 & 26.24204 & 10.89187 & 3.628154 & 10.71953 & 2425.833 & 0.89582 & 237.7801 & 0.675705 \\
\hline Campanula rotundifolia & 7.580132 & 4999.996 & 33.40872 & 381.4606 & 105.1778 & 127.0592 & 55.12649 & 81.098 & 32.67798 & 15.54051 & 7.544083 & 14.09641 & 2695.556 & 0.825217 & 322.2633 & 2.27733 \\
\hline Campanula trachelium & 3.613251 & 2455.716 & 30.43923 & 477.7585 & 40.97868 & 52.67182 & 32.87018 & 25.03339 & 25.10354 & 9.518891 & 4.733105 & 16.91209 & 1152.286 & 1.740656 & 151.1625 & 0.910008 \\
\hline Capsella bursa-pastoris & 3.187113 & 5127.097 & 111.9725 & 563.138 & 149.6444 & 124.1287 & 31.5471 & 38.97353 & 37.94702 & 16.1972 & 3.998473 & 12.51046 & 4051.549 & 2.19281 & 526.1039 & 0.738468 \\
\hline Cardamine amara & 4.816814 & 7868.009 & 185.0512 & 726.3717 & 127.2617 & 220.3831 & 62.48199 & 80.6525 & 58.77455 & 25.99715 & 7.319522 & 20.9311 & 6297.894 & 1.181649 & 409.4445 & 1.218722 \\
\hline Cardamine flexuosa & 5.159174 & 5741.695 & 101.3516 & 628.9488 & 86.70585 & 209.8881 & 54.2321 & 65.05433 & 50.73987 & 25.28274 & 11.40387 & 19.63261 & 4138.493 & 1.165691 & 369.243 & 1.0043 \\
\hline Cardamine hirsuta & 5.792463 & 5341.903 & 92.68561 & 461.2958 & 126.8957 & 160.6813 & 74.29097 & 75.02358 & 42.31238 & 18.93886 & 529522 & 21.30192 & 90.892 & 1.11543 & 405.633 & 1.18 \\
\hline
\end{tabular}




\begin{tabular}{|c|c|c|c|c|c|c|c|c|c|c|c|c|c|c|c|c|}
\hline Cardamine pratensis & 6.35338 & 6414.129 & 251.005 & 454.0025 & 118.119 & 238.5725 & 51.40481 & 61.13603 & 44.75304 & 23.11326 & 9.061511 & 25.06207 & 4503.111 & 1.190164 & 717.0877 & 0.91007 \\
\hline Carduus crispus & 6.587141 & 4241.137 & 205.4576 & 869.9897 & 105.3237 & 106.9544 & 43.65603 & 47.44026 & 47.49483 & 17.41127 & 6.909193 & 25.89951 & 3848.403 & 1.297168 & 482.464 & 0.968132 \\
\hline Carduus nutans & 2.114806 & 4857.54 & 125.5173 & 359.4998 & 122.5323 & 109.2031 & 37.33329 & 40.08366 & 27.30191 & 16.27646 & 3.792969 & 14.42704 & 2917.36 & 1.662128 & 419.7555 & 0.569552 \\
\hline Carex acuta & 5.074931 & 6570.24 & 188.5009 & 490.2288 & 126.8263 & 204.9067 & 40.43897 & 55.99546 & 35.91655 & 21.38531 & 9.06267 & 25.64794 & 6188.701 & 1.202713 & 609.843 & 1.048271 \\
\hline Carex acutiformis & 6.020723 & 7898.408 & 185.6346 & 432.9774 & 124.8906 & 232.6578 & 61.80874 & 79.26966 & 37.42153 & 27.26847 & 10.46444 & 28.07608 & 5097.422 & 1.778053 & 630.2346 & 1.033827 \\
\hline Carex appropinquata & 4.986419 & 5024.726 & 115.9006 & 586.0969 & 85.06898 & 206.6728 & 38.83961 & 52.14034 & 30.32943 & 20.96445 & 11.11767 & 32.35612 & 3230.37 & 2.350136 & 377.9094 & 0.620581 \\
\hline Carex aquatilis & 2.558601 & 3813.731 & 155.37 & 322.7838 & 87.22611 & 189.3375 & 26.07671 & 41.08058 & 26.65118 & 17.07512 & 10.56761 & 18.61974 & 4644.849 & 1.422811 & 448.5022 & 0.458891 \\
\hline Carex arenaria & 8.537242 & 2878.143 & 445.4461 & 261.6339 & 69.62268 & 106.4607 & 70.22408 & 79.49167 & 48.1474 & 13.47189 & 6.909258 & 14.41506 & 2068.682 & 2.159434 & 351.2185 & 2.118836 \\
\hline Carex buxbaumii & 3.070509 & 4827.829 & 70.15389 & 379.8169 & 68.45514 & 181.1108 & 35.43807 & 35.03646 & 20.60654 & 17.30008 & 4.601375 & 9.309443 & 3431.358 & 0.630576 & 300.5673 & 0.511033 \\
\hline Carex caryophyllea & 1.903225 & 4050.965 & 36.76492 & 237.7254 & 90.5294 & 105.8527 & 58.46612 & 55.05634 & 34.13638 & 14.91751 & 3.934825 & 9.61044 & 2219.431 & 0.670298 & 273.2577 & 0.916462 \\
\hline Carex curta & 7.087039 & 9017.913 & 135.805 & 492.8953 & 120.5868 & 291.174 & 66.15641 & 94.70051 & 37.83099 & 30.91359 & 9.233554 & 25.67895 & 3819.779 & 2.565237 & 756.4937 & 0.93208 \\
\hline Carex diandra & 3.191837 & 5097.72 & 106.1225 & 387.8307 & 113.1938 & 154.5049 & 24.01242 & 34.86272 & 19.74984 & 17.7163 & 7.936573 & 16.51324 & 2640.03 & 1.351722 & 640.4185 & 0.558973 \\
\hline Carex distans & 3.643217 & 6623.47 & 583.159 & 370.068 & 117.4977 & 180.0585 & 32.70573 & 36.04531 & 33.87683 & 18.55119 & 5.245054 & 12.68865 & 4325.183 & 0.903146 & 264.7741 & 0.741257 \\
\hline Carex disticha & 3.481877 & 4955.343 & 278.3809 & 344.0564 & 111.6339 & 189.7406 & 33.16759 & 38.69893 & 32.11212 & 22.27367 & 5.762907 & 13.76287 & 4643.023 & 0.77205 & 678.7461 & 0.705843 \\
\hline Carex echinata & 7.750849 & 7778.611 & 91.58281 & 499.4647 & 81.00306 & 253.3973 & 47.65797 & 67.62564 & 31.7836 & 24.64159 & 9.664886 & 12.39303 & 3827.117 & 1.959157 & 527.7781 & 0.877483 \\
\hline Carex elata & 5.466905 & 8552.593 & 119.1384 & 483.8257 & 118.1626 & 256.0602 & 63.83238 & 91.39267 & 45.00238 & 28.31232 & 9.322394 & 33.40266 & 4744.234 & 1.759795 & 711.2355 & 0.867784 \\
\hline Carex elongata & 4.321779 & 4976.554 & 67.82459 & 282.1901 & 82.49636 & 224.3331 & 59.66011 & 83.80595 & 38.32211 & 20.69244 & 7.020086 & 19.57003 & 3312.871 & 1.19721 & 307.2107 & 0.865294 \\
\hline Carex flacca & 6.12937 & 6935.277 & 608.9873 & 416.5612 & 108.935 & 253.7179 & 77.47113 & 73.7438 & 56.12007 & 22.07382 & 4.281322 & 13.49422 & 4458.172 & 0.714423 & 349.4071 & 1.459334 \\
\hline Carex hirta & 5.04579 & 8379.65 & 256.6891 & 520.2966 & 148.3562 & 152.6568 & 50.73977 & 62.58004 & 38.54369 & 22.81458 & 5.490915 & 15.33433 & 5741.728 & 0.948031 & 551.9252 & 1.038685 \\
\hline Carex hostiana & 2.922094 & 3522.914 & 68.29254 & 327.6882 & 55.46135 & 146.8369 & 27.21069 & 31.41843 & 16.01679 & 15.46662 & 3.575798 & 6.855322 & 3193.84 & 0.690723 & 227.9598 & 0.543028 \\
\hline Carex lasiocarpa & 4.995334 & 7293.938 & 122.4892 & 393.7773 & 124.6152 & 167.8981 & 29.50787 & 41.61765 & 26.83114 & 22.01031 & 9.20421 & 20.6445 & 3566.592 & 2.350581 & 650.4065 & 0.751137 \\
\hline Carex ligerica & 2.282975 & 3536.528 & 28.85434 & 165.9289 & 96.55149 & 103.6472 & 53.59839 & 73.33449 & 41.04752 & 13.57247 & 3.636829 & 8.750591 & 1843.878 & 0.898738 & 269.6761 & 2.072693 \\
\hline Carex muricata & 10.14791 & 8199.278 & 440.0956 & 733.5952 & 156.0564 & 243.0645 & 90.77234 & 103.2591 & 63.66072 & 28.25543 & 10.96513 & 32.06421 & 6882.402 & 1.111036 & 717.1701 & 2.341221 \\
\hline Carex nigra & 9.416023 & 8990.742 & 457.4416 & 480.1341 & 126.2542 & 267.7431 & 57.59287 & 82.57872 & 39.79164 & 28.66569 & 9.490563 & 14.33372 & 5096.076 & 1.781275 & 718.0105 & 1.254815 \\
\hline Carex nigra $\mathrm{x}$ trinervis & 4.721666 & 7111.363 & 344.8337 & 242.328 & 118.1802 & 144.0374 & 19.58498 & 41.29952 & 20.00677 & 11.42653 & 6.207126 & 12.51878 & 3262.835 & 2.039451 & 509.7638 & 0.99885 \\
\hline Carex oederi & 6.424305 & 7239.697 & 716.246 & 573.0299 & 127.0054 & 218.4507 & 40.75032 & 64.02845 & 38.42021 & 19.43286 & 5.570114 & 17.46589 & 6333.581 & 0.730531 & 368.2424 & 2.047964 \\
\hline Carex oederi s. oederi & 5.197935 & 4904.649 & 544.9691 & 413.74 & 83.24394 & 179.9564 & 36.34122 & 61.05129 & 33.44533 & 18.68666 & 5.033141 & 12.98126 & 4676.265 & 0.915991 & 281.8876 & 1.627616 \\
\hline Carex oederi s. oedocarpa & 4.851311 & 6167.108 & 465.7201 & 461.7863 & 80.29416 & 173.985 & 40.77432 & 50.63073 & 34.98388 & 19.09568 & 5.318062 & 10.77953 & 4297.978 & 0.924827 & 360.4192 & 0.877099 \\
\hline Carex otrubae & 3.829653 & 6261.648 & 526.5384 & 482.2797 & 94.86842 & 151.6796 & 34.16074 & 43.60076 & 36.15446 & 27.41765 & 5.58985 & 20.59021 & 7837.627 & 0.969492 & 517.6434 & 0.9074 \\
\hline Carex ovalis & 5.723254 & 9008.044 & 106.4281 & 430.4192 & 120.9445 & 178.1192 & 54.80265 & 73.77177 & 30.96131 & 25.1004 & 4.76792 & 11.77216 & 4705.638 & 1.206465 & 392.127 & 0.978537 \\
\hline Carex pallescens & 6.311 & 6841.782 & 60.48709 & 624.3282 & 73.44438 & 173.9569 & 62.32006 & 76.29957 & 42.44315 & 23.62583 & 7.640376 & 25.69468 & 3872.293 & 2.003093 & 243.6196 & 1.010693 \\
\hline Carex panicea & 8.108389 & 7549.973 & 344.6368 & 445.9767 & 94.73905 & 238.5175 & 52.37134 & 68.29779 & 31.78254 & 25.19345 & 5.618205 & 10.77723 & 5350.274 & 1.192274 & 402.897 & 1.144678 \\
\hline Carex paniculata & 5.18494 & 7909.883 & 186.7272 & 433.1012 & 125.2603 & 224.8247 & 57.9494 & 78.09365 & 35.70489 & 25.29508 & 8.926885 & 33.83385 & 3901.478 & 2.1452 & 706.1344 & 1.017739 \\
\hline Carex pilulifera & 7.877735 & 1931.163 & 28.77304 & 837.8209 & 53.48562 & 80.83038 & 60.79089 & 77.24736 & 52.2483 & 13.81431 & 10.87532 & 10.27736 & 2456.35 & 3.174322 & 378.6168 & 1.176255 \\
\hline Carex pseudocyperus & 4.922115 & 7991.315 & 186.9272 & 399.5067 & 131.5735 & 231.5299 & 58.00921 & 82.82989 & 44.29674 & 23.81052 & 9.400132 & 23.3884 & 5310.86 & 1.215965 & 689.7342 & 1.027112 \\
\hline Carex pulicaris & 3.324296 & 4482.895 & 82.15184 & 415.8314 & 57.49001 & 149.368 & 30.64148 & 40.26314 & 18.31633 & 20.01557 & 3.646719 & 7.717575 & 3772.315 & 0.677776 & 250.0673 & 0.650371 \\
\hline Carex remota & 4.337793 & 3998.656 & 73.36386 & 324.6912 & 74.5053 & 157.7168 & 48.83383 & 60.93435 & 44.9776 & 17.07598 & 7.809746 & 17.90395 & 3214.381 & 1.374529 & 278.387 & 0.945763 \\
\hline Carex riparia & 5.499837 & 7916.688 & 274.8734 & 570.9794 & 132.5646 & 237.261 & 58.67966 & 78.45078 & 43.08329 & 26.48021 & 10.60256 & 28.32818 & 5535.831 & 1.566954 & 668.8415 & 1.109767 \\
\hline Carex rostrata & 8.044143 & 8278.833 & 171.4568 & 416.4233 & 140.7363 & 189.8903 & 33.59022 & 44.15257 & 31.04013 & 26.48925 & 9.11318 & 22.30676 & 4803.099 & 2.104989 & 500.6664 & 1.037806 \\
\hline Carex spicata & 5.614842 & 7056.141 & 127.2316 & 435.807 & 114.9058 & 132.442 & 43.02478 & 53.88074 & 43.12382 & 21.06664 & 4.900689 & 14.57267 & 4906.24 & 0.818818 & 366.7836 & 0.989264 \\
\hline Carex sylvatica & 3.729006 & 2758.827 & 34.20535 & 379.1102 & 47.84919 & 64.87154 & 34.10336 & 28.29636 & 24.42032 & 9.817749 & 5.044787 & 14.03296 & 1391.862 & 1.603801 & 145.5415 & 1.26337 \\
\hline Carex trinervis & 6.679536 & 9376.831 & 505.8098 & 401.8395 & 139.477 & 347.7506 & 65.60058 & 120.0102 & 38.00583 & 27.46388 & 4.730035 & 13.25277 & 5288.716 & 1.218276 & 360.458 & 1.345686 \\
\hline Carex vesicaria & 5.563237 & 7991.199 & 132.4397 & 462.7213 & 134.8492 & 241.4052 & 52.21279 & 74.98577 & 45.42762 & 25.68618 & 9.350958 & 23.6048 & 4756.062 & 1.781107 & 522.3128 & 0.964462 \\
\hline Carlina vulgaris & 3.570561 & 3127.752 & 45.75152 & 281.3109 & 74.3358 & 94.3145 & 46.07501 & 46.88414 & 30.05321 & 14.00804 & 5.141064 & 11.72391 & 1856.498 & 0.563862 & 259.898 & 0.938919 \\
\hline Carpinus betulus & 4.649172 & 2892.222 & 33.94636 & 496.0545 & 57.63523 & 86.8977 & 33.27853 & 41.49633 & 28.34147 & 10.63092 & 9.347994 & 21.59383 & 1266.323 & 3.232308 & 177.6958 & 1.14993 \\
\hline Carum carvi & 1.476317 & 3609.996 & 54.74714 & 297.7992 & 71.51862 & 61.64912 & 24.67779 & 27.13452 & 19.57367 & 17.4601 & 2.167891 & 5.752199 & 01.791 & 0.666868 & 5.4788 & \\
\hline
\end{tabular}




\begin{tabular}{|c|c|c|c|c|c|c|c|c|c|c|c|c|c|c|c|c|}
\hline Centaurea cyanus & 3.643836 & 3727.012 & 74.8549 & 534.7347 & 112.6923 & 100.1915 & 27.6397 & 37.52988 & 41.76744 & 10.04198 & 2.91443 & 11.01705 & 2366.716 & 2.581832 & 311.5723 & 1.034307 \\
\hline Centaurea jacea & 4.474746 & 6078.097 & 73.61537 & 409.0391 & 92.1015 & 136.5764 & 49.91862 & 54.13378 & 34.48227 & 25.37866 & 4.933018 & 12.52852 & 5706.889 & 0.785058 & 346.6477 & 1.277664 \\
\hline Centaurea scabiosa & 1.656692 & 3005.789 & 43.75445 & 501.9518 & 69.00757 & 80.3512 & 34.97955 & 32.96205 & 32.46912 & 8.824057 & 3.785593 & 10.75127 & 2026.66 & 0.532784 & 299.478 & 0.382322 \\
\hline Centaurium erythraea & 4.989057 & 5167.421 & 792.5633 & 446.3762 & 107.1705 & 226.0895 & 52.5317 & 56.17221 & 41.68402 & 20.96947 & 5.377852 & 16.40783 & 3796.396 & 0.746114 & 362.3947 & 1.528362 \\
\hline Centaurium littorale & 3.923851 & 7431.806 & 603.3216 & 313.7809 & 121.8813 & 272.0877 & 56.48047 & 58.58968 & 48.60915 & 22.58451 & 6.892748 & 13.69323 & 3495.416 & 0.817875 & 336.7772 & 1.0252 \\
\hline Centaurium pulchellum & 2.992742 & 7827.74 & 608.5898 & 398.2535 & 148.4283 & 275.3843 & 46.25251 & 44.78941 & 47.07344 & 21.92633 & 5.928306 & 15.96163 & 2842.698 & 1.215135 & 275.0752 & 0.770148 \\
\hline Cerastium arvense & 3.365995 & 4149.895 & 52.85565 & 247.2745 & 110.8088 & 103.0863 & 54.52676 & 57.20718 & 29.71306 & 15.81751 & 4.621263 & 12.24504 & 2294.58 & 1.45093 & 325.64 & 1.007643 \\
\hline Cerastium diffusum & 1.496965 & 3053.723 & 753.7391 & 127.1886 & 80.89437 & 133.0929 & 80.40401 & 83.38015 & 71.13488 & 14.57267 & 1.952594 & 12.85854 & 1679.997 & 0.374326 & 275.8483 & 0.831516 \\
\hline Cerastium fontanum & 6.884967 & 8376.981 & 299.7579 & 475.815 & 136.5138 & 156.521 & 54.07372 & 72.68137 & 30.83788 & 26.02973 & 5.935607 & 15.32327 & 5045.469 & 1.346355 & 476.8201 & 1.687406 \\
\hline Cerastium fontanum s. holosteoides & 4.837127 & 6773.504 & 135.195 & 376.5275 & 141.2358 & 149.5916 & 26.55595 & 33.70897 & 19.62912 & 19.26117 & 3.125361 & 11.54135 & 4824.441 & 0.538365 & 580.6016 & 0.74114 \\
\hline Cerastium fontanum s. vulgare & 4.264609 & 7905.118 & 360.9481 & 468.8564 & 139.469 & 162.3429 & 49.0031 & 59.95013 & 30.5059 & 26.56213 & 5.299997 & 13.90107 & 5937.852 & 1.113131 & 588.1749 & 1.16626 \\
\hline Cerastium glomeratum & 4.86028 & 8745.943 & 112.1629 & 516.068 & 174.8063 & 182.0143 & 52.56348 & 66.70668 & 44.03393 & 32.12004 & 5.028834 & 17.22445 & 6874.508 & 2.282964 & 795.6515 & 1.079158 \\
\hline Cerastium semidecandrum & 2.44445 & 2739.839 & 58.89439 & 168.1777 & 59.58492 & 97.77941 & 62.62915 & 54.04669 & 28.33869 & 12.30719 & 3.679809 & 11.14357 & 1722.901 & 1.620734 & 286.3297 & 1.305502 \\
\hline Ceratocapnos claviculata & 6.223567 & 2539.54 & 37.23294 & 501.9613 & 63.78915 & 94.19926 & 57.32716 & 76.71878 & 37.56909 & 16.45612 & 9.632552 & 24.02457 & 2344.171 & 2.31345 & 488.119 & 1.293081 \\
\hline Ceratophyllum demersum & 3.412445 & 5083.168 & 209.5222 & 597.4196 & 71.1933 & 184.1227 & 19.17827 & 25.80051 & 23.59215 & 18.09788 & 6.16387 & 25.74276 & 4525.998 & 1.204773 & 427.1799 & 0.709884 \\
\hline Chaenorhinum minus & 2.437861 & 3821.164 & 123.9536 & 849.7889 & 131.8053 & 110.6507 & 25.53447 & 29.53154 & 33.76043 & 11.39702 & 3.224757 & 14.70212 & 2508.489 & 1.286957 & 409.8825 & 0.56296 \\
\hline Chaerophyllum temulum & 5.626994 & 2819.677 & 38.35982 & 699.1554 & 66.50782 & 72.3412 & 32.79725 & 32.01655 & 23.83201 & 8.923261 & 7.117947 & 18.54481 & 1837.147 & 1.309149 & 213.715 & 1.204999 \\
\hline Chamerion angustifolium & 8.085356 & 3388.356 & 156.4881 & 972.913 & 80.09522 & 124.0667 & 61.39426 & 83.44398 & 53.73184 & 17.3879 & 14.24923 & 31.1038 & 3803.635 & 2.674351 & 490.7871 & 1.79587 \\
\hline Chelidonium majus & 7.142802 & 3113.297 & 55.02459 & 996.0291 & 84.66234 & 91.06842 & 31.72919 & 40.26845 & 19.05463 & 9.098927 & 9.723478 & 22.71424 & 2246.984 & 2.195967 & 452.1661 & 2.108925 \\
\hline Chenopodium album & 5.096714 & 4560.921 & 300.8929 & 657.6613 & 139.0756 & 129.4553 & 34.75242 & 45.78313 & 48.35018 & 16.76962 & 4.067714 & 20.03977 & 3337.367 & 2.273123 & 463.9592 & 1.326137 \\
\hline Chenopodium ficifolium & 2.699898 & 5201.584 & 402.1105 & 760.8398 & 137.6994 & 110.5067 & 32.65863 & 41.37728 & 47.871 & 17.25849 & 4.749671 & 15.05689 & 4103.084 & 1.225817 & 532.3304 & 0.859748 \\
\hline Chenopodium glaucum & 2.442922 & 7974.187 & 525.2799 & 659.31 & 106.0914 & 144.2021 & 38.74007 & 45.84387 & 50.48425 & 37.97885 & 6.015099 & 10.90816 & 8060.215 & 1.807431 & 381.9103 & 0.936277 \\
\hline Chenopodium polyspermum & 5.096252 & 6070.667 & 261.4787 & 666.7346 & 140.0862 & 151.592 & 45.33324 & 62.01074 & 57.35519 & 26.53236 & 7.147652 & 18.71941 & 5927.862 & 1.564249 & 655.5676 & 1.226368 \\
\hline Chenopodium rubrum & 3.77837 & 8053.382 & 653.8453 & 638.199 & 141.7003 & 199.4975 & 55.19325 & 57.9279 & 66.7298 & 33.61758 & 7.481864 & 17.45067 & 8513.494 & 1.738345 & 736.0037 & 0.928723 \\
\hline Chrysanthemum segetum & 3.013212 & 3120.065 & 96.97799 & 682.7531 & 85.94753 & 91.94536 & 27.22073 & 36.2475 & 37.35319 & 11.42525 & 2.943609 & 13.7208 & 2582.373 & 1.781568 & 368.5537 & 0.682803 \\
\hline Chrysosplenium alternifolium & 4.106593 & 5021.858 & 78.75352 & 507.729 & 62.17912 & 175.0668 & 50.96165 & 59.87945 & 56.45399 & 25.81767 & 6.027366 & 18.94186 & 4725.29 & 0.932987 & 212.5152 & .719043 \\
\hline Chrysosplenium oppositifolium & 3.997372 & 5370.219 & 64.06249 & 471.2193 & 59.61125 & 172.4566 & 48.88711 & 61.10956 & 44.41328 & 26.22855 & 5.030951 & 21.20816 & 4084.561 & 1.033437 & 269.3328 & 0.760633 \\
\hline Cichorium intybus & 1.403675 & 4013.433 & 65.70428 & 372.1287 & 84.27454 & 70.3218 & 25.66291 & 27.10995 & 20.91477 & 11.63213 & 2.193918 & 8.762705 & 2397.98 & 0.89962 & 261.6782 & 0.332398 \\
\hline Cicuta virosa & 3.067394 & 5019.881 & 167.0226 & 394.4828 & 92.2992 & 151.0389 & 24.60101 & 39.6805 & 22.82023 & 21.72362 & 9.268542 & 21.6269 & 5094.488 & 0.974656 & 548.9823 & 0.841496 \\
\hline Circaea lutetiana & 4.384425 & 3089.67 & 53.44397 & 393.9645 & 56.27135 & 105.0287 & 41.39158 & 38.04731 & 39.37139 & 15.60324 & 5.582271 & 16.54674 & 2598.948 & 1.172488 & 168.3488 & 1.008304 \\
\hline Cirsium acaule & 1.415823 & 4650.576 & 33.23955 & 359.0916 & 96.27603 & 136.3121 & 65.71905 & 58.25771 & 23.3267 & 17.47695 & 6.284372 & 13.20975 & 4390.923 & 0.53472 & 323.7536 & 0.733238 \\
\hline Cirsium arvense & 5.566974 & 5699.537 & 517.8702 & 559.6947 & 144.3042 & 145.2082 & 45.61681 & 53.97261 & 44.48545 & 23.06763 & 6.96683 & 21.0806 & 5082.137 & 1.363936 & 575.2049 & 1.130069 \\
\hline Cirsium dissectum & 3.957597 & 4653.945 & 102.558 & 373.5286 & 68.05435 & 143.4205 & 35.46726 & 37.41995 & 26.31794 & 18.33822 & 4.525088 & 8.873301 & 3333.902 & 0.780832 & 309.8867 & 0.723939 \\
\hline Cirsium dissectum $\mathrm{x}$ palustre & 3.145255 & 6803.753 & 119.9514 & 388.8328 & 62.05324 & 266.6786 & 41.91473 & 39.91226 & 34.66677 & 23.77358 & 4.959063 & 9.799937 & 3401.033 & 1.194654 & 259.3327 & 0.615382 \\
\hline Cirsium oleraceum & 5.293023 & 7863.864 & 96.13032 & 865.0442 & 88.12017 & 264.2937 & 77.68821 & 100.9789 & 61.71913 & 31.07801 & 11.07537 & 25.16534 & 6708.704 & 1.941974 & 252.7621 & 1.025899 \\
\hline Cirsium palustre & 5.209819 & 7775.027 & 245.5629 & 535.5549 & 88.48698 & 221.8488 & 52.05804 & 62.77931 & 36.70396 & 23.72854 & 8.899814 & 28.16151 & 4569.593 & 1.562836 & 543.2228 & 0.886225 \\
\hline Cirsium vulgare & 6.397181 & 5396.19 & 502.8083 & 647.9096 & 142.7731 & 146.7396 & 51.45536 & 59.67995 & 47.38912 & 19.89546 & 7.508797 & 24.91363 & 4023.279 & 1.639467 & 469.8361 & 1.387125 \\
\hline Cladium mariscus & 4.866335 & 7960.526 & 251.264 & 462.3396 & 116.1857 & 179.773 & 29.74322 & 50.54039 & 28.10787 & 24.86738 & 8.875225 & 38.48406 & 5256.445 & 2.116545 & 788.3801 & 0.991554 \\
\hline Claytonia perfoliata & 6.288114 & 2072.807 & 44.22495 & 726.68 & 59.49768 & 69.71234 & 33.18488 & 38.81233 & 30.44161 & 9.655937 & 7.576103 & 29.76715 & 2014.146 & 1.086627 & 345.5842 & 1.120105 \\
\hline Clematis vitalba & 6.75286 & 3475.623 & 41.69649 & 700.853 & 57.7755 & 64.64216 & 41.5907 & 34.75922 & 32.69704 & 8.056883 & 6.454076 & 25.3839 & 1444.012 & 1.168206 & 226.0367 & 1.700014 \\
\hline Clinopodium acinos & 1.572721 & 2438.505 & 34.73259 & 175.9163 & 56.88371 & 76.04993 & 47.62268 & 44.94993 & 26.79173 & 12.97106 & 5.428445 & 7.278261 & 1479.933 & 1.236635 & 227.8667 & 0.819106 \\
\hline Clinopodium vulgare & 4.910024 & 4140.278 & 48.08181 & 489.849 & 73.22104 & 86.76473 & 30.86509 & 37.90412 & 27.85666 & 13.72137 & 2.926735 & 15.6037 & 2225.798 & 0.554905 & 262.4558 & 0.695449 \\
\hline Cochlearia danica & 1.629437 & 3658.842 & 737.7586 & 176.4084 & 97.80158 & 152.8819 & 81.10996 & 73.78095 & 40.01263 & 19.09262 & 4.067792 & 25.14236 & 2198.774 & 1.460763 & 336.7733 & 0.884771 \\
\hline Cochlearia officinalis s. officinalis & 5.262463 & 3951.177 & 598.1431 & 924.0769 & 91.3796 & 133.5202 & 21.66318 & 27.30371 & 25.45646 & 14.99874 & 11.69475 & 20.2508 & 5407.639 & 1.434821 & 302.0869 & 0.908867 \\
\hline Colchicum autumnale & 8.669586 & 5087.567 & 36.24885 & 1046.901 & 80.87944 & 71.47619 & 66.50119 & 51.96785 & 43.61718 & 14.257 & 6.331087 & 23.438 & 2164.078 & 1.377043 & 245.3312 & 1.377148 \\
\hline Convallaria majalis & 5.217279 & 2393.618 & 29.02355 & 583.8426 & 53.23411 & 82.83834 & 30.5891 & 43.92022 & 28.38257 & 10.40476 & 10.07133 & 28.75559 & 05.627 & 2.582004 & 43.763 & \\
\hline
\end{tabular}




\begin{tabular}{|c|c|c|c|c|c|c|c|c|c|c|c|c|c|c|c|c|}
\hline Convolvulus arvensis & 3.229263 & 5243.269 & 122.8966 & 486.9807 & 156.5297 & 123.6458 & 42.43881 & 47.13481 & 43.30022 & 16.61342 & 4.337223 & 16.42091 & 3320.821 & 1.679628 & 491.505 & 0.61637 \\
\hline Corispermum intermedium & 1.723693 & 3921.999 & 389.804 & 271.1698 & 166.4728 & 121.678 & 42.10724 & 50.66435 & 52.34227 & 11.42391 & 1.809066 & 26.69017 & 2500.429 & 1.15075 & 440.4711 & 0.881506 \\
\hline Cornus sanguinea & 5.827023 & 3149.313 & 53.55496 & 736.5593 & 58.03524 & 80.81901 & 40.18016 & 35.32223 & 33.18115 & 10.91177 & 5.982096 & 19.24541 & 2042.354 & 1.519037 & 240.8487 & 1.833714 \\
\hline Coronopus squamatus & 2.57851 & 4118.641 & 564.4126 & 724.3867 & 111.6129 & 133.8279 & 27.94707 & 33.21817 & 43.09159 & 12.98364 & 3.514194 & 10.47419 & 2981.284 & 0.994922 & 284.1691 & 1.099393 \\
\hline Corydalis solida & 3.874733 & 2803.577 & 36.26832 & 526.518 & 53.68485 & 69.23301 & 26.74566 & 26.05895 & 14.43177 & 9.135322 & 4.738197 & 16.59451 & 1236.944 & 1.049341 & 157.4604 & 0.906956 \\
\hline Corylus avellana & 5.028018 & 2913.807 & 35.51429 & 435.425 & 59.19227 & 94.42119 & 37.6861 & 45.26101 & 33.5576 & 12.05051 & 9.511788 & 22.18524 & 1869.92 & 2.543365 & 182.7509 & 1.179274 \\
\hline Corynephorus canescens & 8.475771 & 1492.567 & 30.67414 & 159.9033 & 32.30237 & 50.94277 & 51.22936 & 68.98397 & 32.56841 & 8.781989 & 3.465364 & 9.260794 & 1234.699 & 0.805058 & 210.239 & 2.118764 \\
\hline Crataegus laevigata & 4.545845 & 2840.358 & 36.33054 & 390.3546 & 56.23311 & 90.79189 & 31.07902 & 34.40957 & 32.92047 & 10.16065 & 7.078132 & 18.15744 & 1981.23 & 1.591572 & 148.8789 & 0.953859 \\
\hline Crataegus monogyna & 6.728931 & 3631.383 & 71.60752 & 963.3811 & 79.41528 & 116.5715 & 44.09017 & 50.78324 & 41.36081 & 17.39772 & 7.978207 & 27.56026 & 3817.793 & 2.348978 & 459.532 & 1.834982 \\
\hline Crepis biennis & 2.327667 & 8039.782 & 54.73411 & 374.2964 & 75.48749 & 72.18438 & 29.50931 & 33.42727 & 25.00717 & 13.52746 & 2.730123 & 9.114577 & 2723.382 & 0.681968 & 234.0261 & 0.558873 \\
\hline Crepis capillaris & 3.836396 & 5023.654 & 572.8648 & 339.8259 & 134.6478 & 132.6254 & 49.32992 & 56.10563 & 31.77065 & 16.69868 & 4.171098 & 14.27653 & 3216.965 & 1.377296 & 412.6787 & 1.069024 \\
\hline Crepis paludosa & 5.531659 & 7290.282 & 144.6802 & 492.733 & 96.94928 & 128.7775 & 52.85588 & 62.77354 & 38.89152 & 23.02312 & 5.468122 & 29.89106 & 3942.371 & 1.194503 & 286.8338 & 0.872691 \\
\hline Crepis tectorum & 3.496194 & 4203.499 & 70.02642 & 216.3056 & 130.74 & 131.9549 & 57.28134 & 62.51514 & 42.90707 & 12.34995 & 1.796123 & 12.92015 & 2599.815 & 3.540892 & 491.9308 & 1.021364 \\
\hline Crepis vesicaria s. taraxacifolia & 1.605165 & 3047.144 & 73.41998 & 277.6204 & 80.48742 & 84.49854 & 28.90892 & 32.4766 & 23.38813 & 11.2337 & 3.248054 & 8.840014 & 2303.283 & 0.595428 & 254.8896 & 0.496452 \\
\hline Cruciata laevipes & 7.59992 & 4225.259 & 59.92086 & 630.1695 & 77.35703 & 82.1559 & 37.20083 & 43.18668 & 42.17351 & 14.50556 & 6.963246 & 32.19957 & 2833.321 & 1.303622 & 340.5633 & 1.315819 \\
\hline Cuscuta epithymum & 10.10111 & 2079.602 & 14.83458 & 1087.949 & 50.24753 & 81.9732 & 57.281 & 83.19213 & 31.63797 & 17.39607 & 6.293301 & 5.15231 & 2593.294 & 1.155843 & 282.1495 & 1.913495 \\
\hline Cuscuta europaea & 5.189603 & 3589.204 & 170.4058 & 913.4715 & 98.07066 & 138.0376 & 46.49008 & 53.1418 & 49.56868 & 19.38911 & 5.796314 & 42.18573 & 4257.963 & 0.89937 & 525.4798 & 1.558632 \\
\hline Cynodon dactylon & 1.727899 & 4235.539 & 109.6552 & 238.1644 & 104.028 & 103.9647 & 41.49608 & 43.90367 & 30.41512 & 14.14079 & 2.782733 & 10.25648 & 2457.712 & 1.314721 & 410.7468 & 0.53902 \\
\hline Cynoglossum officinale & 5.625077 & 2659.738 & 239.974 & 351.6373 & 78.93347 & 118.3108 & 50.88293 & 44.14875 & 27.31762 & 12.25991 & 7.490265 & 26.54738 & 1923.427 & 1.003335 & 339.6641 & 0.882381 \\
\hline Cynosurus cristatus & 2.744019 & 5688.828 & 352.6317 & 373.0627 & 90.03637 & 102.3803 & 27.49623 & 34.42652 & 23.80869 & 19.63114 & 3.824623 & 9.596672 & 4098.706 & 0.560224 & 372.0475 & 0.803918 \\
\hline Cytisus scoparius & 9.821992 & 3072.909 & 33.8318 & 978.4472 & 73.57464 & 101.376 & 55.99657 & 76.53605 & 37.62547 & 15.48261 & 14.50159 & 13.61879 & 2743.594 & 3.626102 & 394.8594 & 1.355716 \\
\hline Dactylis glomerata & 7.542967 & 4932.269 & 89.94947 & 600.1604 & 113.2589 & 106.6825 & 39.51138 & 48.33501 & 46.00626 & 14.33051 & 6.332904 & 21.52107 & 3433.364 & 1.748635 & 387.9114 & 1.54959 \\
\hline Dactylorhiza incarnata & 3.754739 & 5963.428 & 423.0806 & 415.9139 & 96.1307 & 164.8282 & 30.33141 & 32.45674 & 29.62312 & 21.63409 & 5.302565 & 12.28984 & 5557.532 & 1.053583 & 428.7394 & 1.21555 \\
\hline Dactylorhiza maculata & 6.226571 & 7495.865 & 54.78659 & 524.4651 & 82.58532 & 203.6047 & 62.05109 & 85.82895 & 35.128 & 37.88311 & 4.687688 & 12.23357 & 6505.261 & 0.796501 & 359.9048 & 1.862041 \\
\hline Dactylorhiza maculata s. fuchsii & 5.891967 & 3741.754 & 412.3078 & 359.1485 & 60.35142 & 111.1643 & 45.55442 & 39.87213 & 52.46897 & 8.989393 & 4.898836 & 25.44827 & 1574.902 & 0.505641 & 139.1046 & 1.384718 \\
\hline Dactylorhiza majalis & 4.320547 & 6554.146 & 188.4589 & 407.3042 & 108.8021 & 227.0827 & 27.81462 & 34.89702 & 25.54924 & 24.1886 & 7.004159 & 11.875 & 5096.268 & 0.838093 & 564.4473 & 1.131831 \\
\hline Dactylorhiza majalis s. majalis & 5.542674 & 5295.864 & 186.7997 & 394.9493 & 68.89152 & 203.7406 & 25.86188 & 31.68144 & 24.23983 & 17.02013 & 8.020478 & 12.11776 & 3698.167 & 0.760336 & 522.2995 & 0.942661 \\
\hline Dactylorhiza majalis s. praterrmissa & 4.064821 & 6154.648 & 244.8473 & 437.5683 & 86.62891 & 201.6779 & 31.34476 & 39.53014 & 24.29548 & 20.8755 & 8.299739 & 14.93042 & 4612.162 & 1.400843 & 740.7629 & 0.914929 \\
\hline Danthonia decumbens & 8.196607 & 5788.257 & 51.60159 & 764.1125 & 93.27769 & 210.5525 & 79.92006 & 111.6452 & 37.021 & 33.2846 & 6.208526 & 9.428508 & 6496.719 & 1.021279 & 318.6002 & 1.361984 \\
\hline Daucus carota & 3.199672 & 5395.964 & 514.0594 & 430.4209 & 119.0977 & 124.603 & 50.2237 & 53.12058 & 31.6643 & 20.56548 & 4.697859 & 12.90489 & 3720.278 & 0.863562 & 455.0162 & 0.681857 \\
\hline Deschampsia cespitosa & 8.009509 & 7986.881 & 81.51794 & 601.5424 & 138.8814 & 157.2921 & 60.54613 & 76.27825 & 48.37887 & 23.58285 & 9.42576 & 29.03973 & 4020.967 & 2.104566 & 316.7458 & 1.282579 \\
\hline Deschampsia flexuosa & 7.153575 & 1549.836 & 32.24999 & 691.5422 & 50.41953 & 74.56554 & 54.15045 & 67.55507 & 40.88267 & 13.69944 & 11.38192 & 12.79259 & 3175.314 & 2.577817 & 635.7905 & 1.08066 \\
\hline Descurainia sophia & 2.537692 & 4631.317 & 145.7849 & 376.191 & 159.5883 & 148.6431 & 58.77936 & 53.10083 & 41.23949 & 10.13198 & 2.440828 & 24.80547 & 2150.225 & 0.881008 & 839.893 & 0.614733 \\
\hline Dianthus deltoides & 3.959002 & 2968.43 & 28.15191 & 185.5545 & 64.46695 & 76.04078 & 39.52607 & 48.79827 & 26.72046 & 15.68171 & 2.914508 & 6.870469 & 2629.048 & 0.996509 & 291.0947 & 0.677541 \\
\hline Digitalis purpurea & 6.26947 & 2843.418 & 35.49286 & 642.8202 & 66.17524 & 103.4058 & 52.47985 & 71.14216 & 37.37937 & 14.2704 & 9.368935 & 26.94439 & 2221.366 & 2.377582 & 268.2147 & 1.363068 \\
\hline Digitaria ischaemum & 4.389178 & 3754.841 & 99.5272 & 336.985 & 113.6061 & 139.9184 & 49.41701 & 68.68618 & 59.60401 & 16.40856 & 2.679326 & 14.10048 & 3348.05 & 3.679893 & 570.5151 & 1.027167 \\
\hline Diplotaxis tenuifolia & 2.238556 & 4164.538 & 103.4338 & 270.5171 & 137.8889 & 145.5677 & 55.62795 & 53.5349 & 39.75989 & 15.59376 & 3.228629 & 18.67442 & 2659.367 & 1.794294 & 476.852 & 0.696534 \\
\hline Dipsacus fullonum & 7.858807 & 4932.009 & 295.008 & 566.7013 & 118.8918 & 106.2481 & 47.72423 & 51.63844 & 49.13229 & 13.61237 & 7.333835 & 25.88199 & 2781.645 & 1.495613 & 257.9692 & 1.302888 \\
\hline Drosera intermedia & 8.349324 & 3920.689 & 12.51413 & 679.9062 & 65.82978 & 162.2324 & 49.36119 & 51.47834 & 29.96514 & 19.51993 & 6.926814 & 5.707271 & 3672.803 & 1.420812 & 240.4187 & 0.822517 \\
\hline Drosera rotundifolia & 9.399325 & 6681.614 & 144.1012 & 470.9052 & 83.81258 & 324.4431 & 63.14348 & 95.14058 & 27.5298 & 26.35308 & 10.86279 & 10.23452 & 4837.495 & 2.690934 & 671.1877 & 0.876854 \\
\hline Dryopteris carthusiana & 7.440006 & 3944.849 & 82.40374 & 563.1496 & 87.9759 & 202.8712 & 80.35162 & 103.9104 & 51.152 & 23.42151 & 8.54531 & 31.79758 & 4151.007 & 2.094431 & 548.9636 & 1.484276 \\
\hline Dryopteris carthusiana + D. dilatata & 6.279211 & 5132.035 & 290.9772 & 652.0048 & 109.3609 & 321.7202 & 110.318 & 142.8751 & 88.49807 & 34.45898 & 9.93025 & 39.9347 & 5454.292 & 2.892685 & 381.9678 & 1.310516 \\
\hline Dryopteris cristata & 5.080109 & 6540.498 & 127.439 & 470.3445 & 79.05973 & 262.2131 & 55.20933 & 70.85152 & 27.40097 & 22.70777 & 9.063206 & 24.74996 & 3305.299 & 1.800763 & 615.593 & 0.831722 \\
\hline Dryopteris dilatata & 6.722932 & 3328.307 & 63.22945 & 537.6411 & 78.37346 & 141.7596 & 67.13654 & 87.92986 & 47.98295 & 19.66456 & 8.283106 & 29.21881 & 3534.977 & 2.066872 & 464.5304 & 1.448027 \\
\hline Dryopteris filix-mas & 6.173049 & 3198.828 & 65.82793 & 855.4837 & 66.74958 & 105.2576 & 46.72411 & 57.19486 & 41.61561 & 15.23075 & 9.917562 & 27.4877 & 3094.861 & 1.998649 & 399.1694 & 371914 \\
\hline Echinochloa crus-galli & 3.550341 & 5116.578 & 118.8398 & 650.1808 & 114.8305 & 58.1022 & 54.36306 & 4.36987 & 70.15497 & 16.74711 & 3.11446 & 14.7586 & 89.105 & 14627 & 35.8497 & \\
\hline
\end{tabular}




\begin{tabular}{|c|c|c|c|c|c|c|c|c|c|c|c|c|c|c|c|c|}
\hline Echinodorus ranunculoides & 4.574697 & 5636.098 & 432.5795 & 629.0335 & 105.6314 & 184.0995 & 15.04558 & 28.67894 & 16.24626 & 15.16715 & 5.413244 & 13.30562 & 4019.268 & 0.705359 & 494.4782 & 1.169138 \\
\hline Echium vulgare & 2.103812 & 3128.641 & 65.50281 & 259.0782 & 97.51492 & 111.115 & 53.40455 & 45.66348 & 32.08744 & 13.51104 & 5.460085 & 21.51026 & 1993.153 & 1.243591 & 337.1174 & 0.670694 \\
\hline Eleocharis acicularis & 4.106085 & 5904.563 & 201.0097 & 288.5272 & 100.0244 & 207.5007 & 32.87521 & 48.30101 & 36.6075 & 24.27706 & 6.712407 & 22.16583 & 6425.638 & 1.074159 & 594.8893 & 1.429059 \\
\hline Eleocharis multicaulis & 9.918696 & 6800.833 & 271.7037 & 935.5917 & 90.44369 & 285.4146 & 14.38536 & 23.58019 & 15.9546 & 19.98846 & 10.79865 & 11.26773 & 3870.934 & 1.450967 & 342.0172 & 1.039475 \\
\hline Eleocharis palustris & 4.655194 & 5634.821 & 372.2524 & 371.7992 & 102.6917 & 179.5738 & 31.50796 & 52.40628 & 33.25775 & 22.6362 & 5.174258 & 17.07671 & 6688.936 & 1.04158 & 762.908 & 1.135285 \\
\hline Eleocharis palustris ag. (incl. E. uniglumis) & 4.746944 & 5152.892 & 446.2801 & 385.9018 & 103.1933 & 202.2658 & 35.46952 & 53.8269 & 38.49278 & 25.02526 & 10.99815 & 18.02192 & 7354.261 & 1.174653 & 869.5392 & 1.460187 \\
\hline Eleocharis quinqueflora & 5.39689 & 5380.653 & 567.9454 & 345.7808 & 106.0249 & 184.0438 & 38.61144 & 62.64662 & 34.36444 & 14.879 & 4.796922 & 12.14694 & 4151.907 & 0.838038 & 293.869 & 1.47224 \\
\hline Eleocharis uniglumis & 4.832369 & 5342.065 & 612.2906 & 403.8769 & 102.533 & 187.2242 & 37.41627 & 52.76417 & 40.46506 & 15.39499 & 5.191956 & 15.49322 & 4941.75 & 0.982294 & 435.9609 & 1.363945 \\
\hline Elodea canadensis & 2.380713 & 3854.471 & 231.8486 & 345.1513 & 77.26351 & 173.2801 & 20.4718 & 20.76482 & 25.41025 & 15.1461 & 11.16471 & 12.3696 & 4367.919 & 0.976608 & 477.8351 & 0.648452 \\
\hline Elymus caninus & 5.281703 & 6540.973 & 98.91407 & 576.6485 & 86.44951 & 249.668 & 106.4252 & 119.3242 & 71.17165 & 38.43689 & 6.941595 & 37.12195 & 8178.546 & 1.841671 & 767.5249 & 1.135595 \\
\hline Elytrigia atherica & 3.374302 & 4974.197 & 733.5114 & 310.0693 & 198.181 & 246.7211 & 64.34698 & 69.48599 & 43.97317 & 25.89829 & 6.194231 & 27.04362 & 3259.44 & 1.670722 & 432.8299 & 0.981682 \\
\hline Elytrigia juncea s. boreoatlantica & 1.21645 & 3966.267 & 768.9131 & 166.2282 & 130.487 & 264.9085 & 94.06186 & 81.62391 & 69.73889 & 21.34745 & 2.188916 & 26.57148 & 2263.252 & 1.2052 & 394.3868 & 0.96158 \\
\hline Elytrigia repens & 6.030006 & 5361.955 & 561.0629 & 583.1749 & 143.7017 & 127.3922 & 40.76837 & 50.33254 & 47.49689 & 19.43397 & 6.169849 & 17.21418 & 4616.128 & 1.754976 & 560.4819 & 1.401679 \\
\hline Empetrum nigrum & 10.31234 & 2520.422 & 130.8232 & 1055.189 & 77.25537 & 147.1752 & 94.41391 & 109.3021 & 54.01427 & 14.30534 & 9.301315 & 8.116619 & 2706.752 & 2.369591 & 403.7919 & 1.579225 \\
\hline Epilobium hirsutum & 4.081015 & 8296.957 & 322.6727 & 841.9797 & 130.1038 & 260.9174 & 58.80266 & 73.41136 & 55.73168 & 28.48808 & 9.037792 & 21.71747 & 7361.473 & 0.945245 & 590.032 & 1.382652 \\
\hline Epilobium montanum & 5.378277 & 4162.334 & 160.8415 & 682.5986 & 81.3305 & 178.7524 & 62.66458 & 69.72017 & 53.48473 & 23.28016 & 5.814485 & 22.76007 & 4757.738 & 1.744376 & 474.196 & 1.401101 \\
\hline Epilobium obscurum & 6.322686 & 8627.893 & 370.5921 & 740.7089 & 127.9589 & 314.5449 & 68.91666 & 98.85291 & 73.14981 & 36.93058 & 13.21198 & 29.64958 & 9367.492 & 1.872545 & 892.5858 & 1.650401 \\
\hline Epilobium palustre & 5.107519 & 7332.127 & 436.8035 & 501.8284 & 132.151 & 209.7254 & 45.68455 & 67.4857 & 34.17302 & 25.71108 & 10.70715 & 30.47271 & 5358.538 & 1.593372 & 817.2266 & 1.081701 \\
\hline Epilobium parviflorum & 5.148081 & 8474.201 & 471.6261 & 617.1205 & 140.0971 & 264.2156 & 54.51369 & 66.12491 & 57.64951 & 31.34991 & 9.173115 & 25.0449 & 7401.156 & 1.104494 & 667.9004 & 1.555967 \\
\hline Epilobium roseum & 5.181691 & 8156.364 & 140.4128 & 894.8706 & 111.8646 & 286.5607 & 52.83956 & 65.88267 & 52.91138 & 39.24255 & 9.310587 & 25.86638 & 8422.245 & 2.889221 & 824.4634 & 2.419723 \\
\hline Epilobium tetragonum & 5.747398 & 8256.739 & 504.5509 & 646.4966 & 129.1223 & 238.1432 & 52.84229 & 68.88113 & 47.77815 & 33.14686 & 10.59293 & 31.96819 & 6554.481 & 1.792688 & 584.1493 & 1.953931 \\
\hline Epipactis helleborine & 7.11857 & 4203.304 & 79.96424 & 1012.175 & 92.23751 & 138.7484 & 46.64476 & 56.34499 & 44.24015 & 21.45087 & 7.498797 & 33.26665 & 3678.778 & 1.921438 & 456.5047 & 1.884588 \\
\hline Epipactis palustris & 4.440088 & 5934.795 & 460.0924 & 409.4248 & 89.48903 & 181.9759 & 42.24364 & 45.83096 & 36.14927 & 21.18 & 4.539877 & 12.2774 & 5582.024 & 0.979439 & 340.9198 & 1.375087 \\
\hline Equisetum arvense & 7.536232 & 5692.177 & 223.2327 & 653.7975 & 123.187 & 127.6444 & 45.96203 & 56.03752 & 51.0737 & 21.36792 & 7.998234 & 22.52394 & 4316.669 & 1.692788 & 402.7852 & 1.500239 \\
\hline Equisetum fluviatile & 4.906374 & 6118.721 & 196.0535 & 446.1835 & 125.3557 & 183.2452 & 35.53631 & 49.71628 & 33.46428 & 20.03347 & 10.76732 & 26.3727 & 5788.242 & 1.523322 & 565.8358 & 0.943456 \\
\hline Equisetum hyemale & 7.538429 & 5069.976 & 92.84252 & 695.735 & 83.44566 & 199.5582 & 61.19438 & 78.77084 & 55.56703 & 25.38568 & 11.78823 & 23.8181 & 4967.403 & 2.798112 & 5.6612 & 1.676277 \\
\hline Equisetum hyemale ag. (incl. E. x moorei) & 9.457543 & 6886.617 & 113.9491 & 812.7121 & 91.15763 & 176.9031 & 69.51579 & 83.6631 & 87.2905 & 30.95232 & 11.56159 & 33.7257 & 6479.826 & 1.905844 & 376.2626 & 2.167222 \\
\hline Equisetum palustre & 5.574434 & 6241.536 & 202.0101 & 502.3304 & 130.7414 & 232.4272 & 46.09006 & 64.00626 & 37.96198 & 25.13774 & 7.42732 & 25.07612 & 6907.397 & 1.377981 & 751.0934 & 1.154211 \\
\hline Equisetum telmateia & 4.906101 & 8004.892 & 97.51519 & 357.2382 & 88.35636 & 156.5559 & 47.85992 & 51.13955 & 41.04078 & 21.40837 & 7.910921 & 23.66241 & 3491.912 & 0.88076 & 265.8578 & 0.908772 \\
\hline Equisetum variegatum & 2.577292 & 7424.289 & 226.6674 & 385.657 & 114.9955 & 171.3967 & 34.87175 & 39.65453 & 26.46393 & 21.49589 & 3.788419 & 11.82542 & 4996.95 & 0.840651 & 393.791 & 0.866291 \\
\hline Equisetum $x$ litorale & 7.352125 & 8811.817 & 248.9076 & 880.0624 & 150.0664 & 306.6408 & 75.96409 & 105.0738 & 63.1964 & 33.18701 & 12.83232 & 34.55596 & 8669.531 & 2.678903 & 904.3194 & 1.581599 \\
\hline Erica tetralix & 8.63517 & 3699.55 & 30.1681 & 1016.644 & 73.85474 & 152.5898 & 99.13974 & 153.4974 & 49.51198 & 23.60568 & 9.214962 & 7.421564 & 4190.575 & 2.526333 & 296.2025 & 1.096115 \\
\hline Erigeron acer & 4.179141 & 3074.619 & 323.5069 & 228.2937 & 78.99614 & 120.2872 & 54.68211 & 54.81224 & 38.37068 & 16.8236 & 5.100509 & 10.67226 & 1866.014 & 1.25312 & 251.1032 & 1.255892 \\
\hline Eriophorum angustifolium & 10.16623 & 8689.521 & 128.0812 & 511.065 & 116.4576 & 308.6559 & 44.61427 & 55.37794 & 32.69897 & 31.49994 & 10.08183 & 10.74901 & 4995.28 & 2.433816 & 929.7323 & 1.076565 \\
\hline Eriophorum vaginatum & 3.617206 & 2804.523 & 24.019 & 631.8102 & 68.52153 & 154.3922 & 102.1748 & 163.8993 & 38.64738 & 22.40465 & 9.865187 & 6.61839 & 5319.239 & 1.853866 & 578.4624 & 0.628088 \\
\hline Erodium cicutarium & 3.938967 & 4089.644 & 75.69764 & 250.4003 & 118.0817 & 132.5035 & 67.9765 & 62.86581 & 37.45392 & 13.63506 & 2.77163 & 16.20517 & 2544.361 & 2.895282 & 475.9611 & 1.167429 \\
\hline Erodium cicutarium s. cicutarium & 4.161318 & 4018.497 & 92.70434 & 298.1212 & 128.7932 & 124.2715 & 37.17882 & 47.06074 & 30.40635 & 11.96285 & 2.819411 & 13.08132 & 2352.866 & 2.845273 & 509.3341 & 1.185093 \\
\hline Erodium cicutarium s. dunense & 1.858748 & 2552.846 & 47.37191 & 171.7156 & 57.16727 & 97.52125 & 61.39563 & 54.02949 & 23.992 & 11.42564 & 4.248637 & 11.84917 & 1473.805 & 1.436398 & 262.4013 & 0.779235 \\
\hline Erodium lebelii & 1.646596 & 1783.139 & 47.59089 & 154.5581 & 41.35399 & 80.1083 & 52.26375 & 45.34769 & 25.11016 & 9.558613 & 4.170288 & 14.02434 & 1341.07 & 1.401105 & 247.481 & 1.042326 \\
\hline Erophila verna & 1.590298 & 3677.806 & 52.368 & 158.0873 & 74.57972 & 114.3909 & 70.55659 & 62.45183 & 37.33736 & 13.89281 & 3.349667 & 10.36597 & 2030.982 & 2.018544 & 325.2377 & 0.964551 \\
\hline Eryngium campestre & 1.832047 & 4726.959 & 67.30345 & 288.3801 & 123.9283 & 128.4276 & 44.23349 & 48.77379 & 27.33849 & 15.81388 & 2.608387 & 12.32269 & 3113.398 & 1.240173 & 428.5054 & 0.495599 \\
\hline Eryngium maritimum & 1.00011 & 3343.513 & 726.8226 & 90.39604 & 92.97531 & 173.27 & 82.07349 & 72.48267 & 45.44295 & 13.23089 & 2.383537 & 19.94915 & 1603.318 & 1.016129 & 280.5192 & 0.974563 \\
\hline Erysimum cheiranthoides & 3.14404 & 4346.135 & 124.1292 & 626.6097 & 122.3155 & 125.6328 & 32.17304 & 42.87317 & 44.09952 & 18.33686 & 3.734181 & 22.52998 & 3718.693 & 2.397907 & 390.7797 & 0.769111 \\
\hline Euonymus europaeus & 5.508424 & 2627.57 & 39.90879 & 811.4726 & 64.45122 & 87.65211 & 33.20646 & 36.47332 & 27.43888 & 14.87206 & 5.521526 & 24.84439 & 2756.565 & 1.012821 & 411.0219 & 1.484497 \\
\hline Eupatorium cannabinum & 4.975925 & 7946.343 & 407.8965 & 599.0892 & 101.7185 & 249.903 & 59.36162 & 70.48578 & 46.67329 & 24.81247 & 9.297414 & 26.94644 & 5867.062 & 1.130516 & 681.6754 & 1.504479 \\
\hline Euphorbia cyparissias & 3.186074 & 3749.946 & 48.16887 & 367.2521 & 88.60335 & 89.75698 & 46.56395 & 51.43987 & 41.83612 & 15.1495 & 327353 & 19.24674 & 2463.78 & 1.546503 & 43.5842 & 0.77993 \\
\hline
\end{tabular}




\begin{tabular}{|c|c|c|c|c|c|c|c|c|c|c|c|c|c|c|c|c|}
\hline Euphorbia esula & 4.596667 & 4325.457 & 133.5533 & 536.7034 & 107.7767 & 94.85775 & 37.25364 & 43.11307 & 44.1681 & 15.76347 & 4.795688 & 19.48387 & 3277.433 & 1.231432 & 368.5062 & 0.672096 \\
\hline Euphorbia esula s. esula & 2.220239 & 3818.526 & 111.4196 & 374.7437 & 96.02609 & 99.68096 & 31.54562 & 35.41719 & 26.82411 & 14.28419 & 4.418275 & 14.69266 & 3388.773 & 0.934431 & 337.1545 & 0.507354 \\
\hline Euphorbia exigua & 2.742772 & 2775.194 & 82.02628 & 610.671 & 119.7832 & 72.37698 & 26.25367 & 28.74245 & 32.47875 & 11.32048 & 2.810875 & 8.765665 & 2075.265 & 1.965946 & 249.9548 & 0.430535 \\
\hline Euphorbia helioscopia & 2.555683 & 3215.79 & 87.72593 & 564.1499 & 114.0705 & 93.92975 & 22.89779 & 28.10589 & 39.85119 & 12.55997 & 3.587623 & 15.7704 & 2286.177 & 1.504467 & 273.7725 & 0.651296 \\
\hline Euphorbia palustris & 5.698459 & 8121.217 & 112.0864 & 408.9807 & 107.2642 & 208.3056 & 48.48887 & 66.0668 & 32.21758 & 28.54478 & 11.15513 & 26.60131 & 4496.555 & 1.236112 & 362.4889 & 1.449241 \\
\hline Euphorbia peplus & 3.598993 & 3962.019 & 108.9242 & 963.9962 & 134.1315 & 98.13164 & 25.44956 & 35.64998 & 47.07241 & 13.08105 & 3.184677 & 27.91043 & 2992.422 & 1.349568 & 518.4427 & 0.690189 \\
\hline Euphrasia stricta & 4.671383 & 6923.296 & 494.1664 & 368.4439 & 108.1854 & 285.1402 & 78.1914 & 80.8212 & 48.05714 & 26.647 & 5.46509 & 11.58401 & 4433.906 & 0.756409 & 344.0104 & 1.393336 \\
\hline Fagus sylvatica & 7.239445 & 2468.439 & 31.38194 & 484.215 & 65.51629 & 99.27289 & 56.19559 & 75.83315 & 30.44622 & 16.72765 & 9.863712 & 32.51959 & 1917.594 & 2.385597 & 683.6541 & 1.38966 \\
\hline Fallopia convolvulus & 7.023464 & 3910.177 & 87.50347 & 687.7007 & 116.2412 & 104.7847 & 44.95742 & 56.97557 & 37.09173 & 11.02907 & 4.806701 & 19.79509 & 2510.789 & 2.596561 & 509.8739 & 1.605372 \\
\hline Fallopia dumetorum & 6.143095 & 2889.033 & 55.23013 & 814.6107 & 65.96351 & 95.77579 & 35.12906 & 43.13837 & 33.66872 & 14.48685 & 9.299974 & 21.50583 & 3156.726 & 2.488178 & 430.3112 & 1.919376 \\
\hline Festuca arenaria & 2.552434 & 2917.255 & 753.2029 & 191.9546 & 65.11716 & 109.4855 & 74.30967 & 69.82735 & 53.62718 & 12.78317 & 4.851407 & 17.77169 & 1787.322 & 1.839347 & 313.2212 & 1.582562 \\
\hline Festuca arundinacea & 4.372098 & 7043.484 & 641.6782 & 489.6778 & 130.0871 & 149.2917 & 41.15183 & 46.84401 & 37.25275 & 18.62667 & 5.508997 & 15.64406 & 4667.786 & 0.993082 & 462.0166 & 1.010978 \\
\hline Festuca cinerea & 7.591305 & 3733.547 & 29.02912 & 208.6349 & 89.68852 & 96.2325 & 58.41281 & 72.42934 & 35.48901 & 13.85757 & 3.83238 & 10.99509 & 2246.957 & 1.921378 & 319.7437 & 1.408427 \\
\hline Festuca filiformis & 10.19047 & 2813.03 & 33.54824 & 364.8822 & 71.66516 & 97.98413 & 68.61187 & 83.28037 & 46.10706 & 13.83417 & 7.489547 & 11.87435 & 2240.987 & 1.779487 & 359.4045 & 2.058686 \\
\hline Festuca gigantea & 4.585037 & 3630.879 & 84.65689 & 311.8882 & 69.09916 & 108.823 & 38.02142 & 40.18275 & 37.08319 & 14.1684 & 5.648653 & 13.4907 & 2691.381 & 1.062288 & 168.4478 & 1.012104 \\
\hline Festuca ovina & 8.165093 & 4000.457 & 24.29702 & 398.2844 & 92.85738 & 122.0737 & 63.39819 & 88.65596 & 55.8979 & 19.88838 & 5.821413 & 11.32403 & 3112.136 & 1.234123 & 448.4132 & 1.459099 \\
\hline Festuca ovina ag. (incl. F. cinerea, F. filiformis) & 10.40977 & 3629.666 & 36.59417 & 579.2233 & 79.79647 & 114.3748 & 87.8306 & 114.1202 & 42.7543 & 28.01772 & 7.414947 & 9.445301 & 3700.265 & 1.085583 & 443.7925 & 1.880768 \\
\hline Festuca pratensis & 2.958826 & 6182.984 & 136.829 & 339.0499 & 99.92821 & 107.1165 & 31.51165 & 38.77816 & 27.42995 & 21.74607 & 4.100444 & 9.669669 & 4146.859 & 0.702279 & 356.3136 & 0.781611 \\
\hline Festuca rubra & 5.282286 & 7551.495 & 749.5521 & 384.6843 & 162.6031 & 204.5135 & 72.94059 & 81.02941 & 43.94263 & 32.35726 & 6.766166 & 15.10773 & 5416.084 & 1.43298 & 524.2703 & 1.788072 \\
\hline Festuca rubra ag. (incl. F. arenaria) & 6.523933 & 7433.388 & 750.0438 & 430.295 & 148.4422 & 215.0129 & 73.21249 & 85.02147 & 44.13339 & 33.03673 & 7.762431 & 16.13069 & 6293.077 & 1.33182 & 568.8376 & 2.169552 \\
\hline Festulolium loliaceum & 4.165521 & 7672.467 & 93.89134 & 363.1202 & 129.1123 & 140.1064 & 36.81037 & 45.52169 & 36.81312 & 31.91292 & 4.812014 & 20.98089 & 4640.183 & 1.274052 & 527.6917 & 1.197696 \\
\hline Filago minima & 4.389764 & 2762.98 & 20.94994 & 213.4747 & 68.12077 & 74.19111 & 46.74082 & 61.21602 & 36.45129 & 12.56396 & 2.634837 & 9.170448 & 1982.705 & 0.538119 & 310.6511 & 0.962716 \\
\hline Filipendula ulmaria & 5.365481 & 7415.519 & 133.3238 & 503.041 & 111.0281 & 218.9433 & 54.4183 & 66.64644 & 44.17038 & 26.05378 & 7.735989 & 29.59973 & 4815.204 & 1.469769 & 460.6144 & 0.844445 \\
\hline Fragaria vesca & 6.712801 & 3152.511 & 66.05711 & 716.97 & 68.94891 & 105.9341 & 43.71364 & 44.75841 & 46.38023 & 15.24695 & 6.650346 & 30.36295 & 2774.938 & 1.696322 & 364.1867 & 1.576316 \\
\hline Fraxinus excelsior & 5.446479 & 3367.59 & 59.37102 & 613.9301 & 66.26924 & 116.645 & 46.06962 & 46.67988 & 41.67751 & 15.409 & 7.895249 & 19.25523 & 2952.498 & 2.156871 & 271.5625 & 1.489858 \\
\hline Fritillaria meleagris & 5.534717 & 7527.578 & 63.28748 & 619.3781 & 93.59812 & 95.37819 & 44.89846 & 54.26374 & 54.84478 & 25.89056 & 4.533845 & 29.90238 & 5080.234 & 0.891287 & 277.7597 & 0.541242 \\
\hline Fumaria officinalis & 3.214784 & 3761.865 & 139.7439 & 653.3545 & 127.1482 & 93.18939 & 30.03573 & 35.9104 & 42.75865 & 12.36353 & 3.46188 & 15.3955 & 2367.311 & 1.817054 & 345.0444 & 0.75971 \\
\hline Galanthus nivalis & 4.813366 & 2727.412 & 32.10751 & 481.8098 & 54.82256 & 62.62757 & 30.80842 & 31.43115 & 14.61363 & 8.685701 & 5.979834 & 18.19432 & 1173.827 & 1.286576 & 199.2491 & 0.982873 \\
\hline Galeopsis bifida & 7.438005 & 7248.835 & 116.1242 & 570.4535 & 106.827 & 292.2233 & 87.25943 & 122.852 & 65.31906 & 39.21315 & 15.74192 & 26.66994 & 5833.531 & 2.059544 & 570.4532 & 1.562126 \\
\hline Galeopsis bifida + G. tetrahit & 6.698862 & 6166.824 & 97.30997 & 511.3052 & 119.1477 & 306.0218 & 88.02321 & 119.8158 & 62.04749 & 39.67691 & 7.223884 & 25.3532 & 4452.503 & 1.902367 & 279.0789 & 1.834581 \\
\hline Galeopsis speciosa & 5.689366 & 4680.648 & 99.56043 & 874.318 & 104.0614 & 191.7077 & 43.95171 & 57.16269 & 48.38319 & 29.20367 & 8.612928 & 33.43074 & 4934.405 & 2.606704 & 564.1614 & 1.583619 \\
\hline Galeopsis tetrahit & 7.737331 & 4204.671 & 88.90158 & 697.6877 & 89.3841 & 160.9714 & 54.97091 & 79.07627 & 48.24554 & 22.47543 & 13.09358 & 27.60938 & 3780.199 & 2.610949 & 318.4686 & 1.701787 \\
\hline Galium aparine & 6.05582 & 4165.033 & 146.4205 & 749.5849 & 86.21346 & 151.0965 & 47.47031 & 53.5489 & 45.9023 & 23.22577 & 9.589931 & 21.18092 & 4539.394 & 1.338073 & 451.1929 & 1.69568 \\
\hline Galium mollugo & 4.975424 & 4945.283 & 62.83214 & 408.0366 & 120.8215 & 137.5703 & 71.66669 & 69.83054 & 37.1995 & 22.14933 & 6.598121 & 19.57266 & 2982.137 & 1.497159 & 402.5027 & 1.263511 \\
\hline Galium odoratum & 3.547805 & 2197.108 & 28.71849 & 352.6405 & 38.5049 & 64.45739 & 27.33934 & 23.54855 & 21.83069 & 8.045657 & 4.171108 & 16.6364 & 808.1938 & 2.496973 & 133.6901 & 0.838322 \\
\hline Galium palustre & 5.281365 & 6951.382 & 273.6938 & 494.429 & 124.283 & 221.2278 & 45.20909 & 65.53515 & 41.01984 & 24.33333 & 10.08216 & 28.65699 & 5356.309 & 1.440026 & 782.478 & 0.991921 \\
\hline Galium pumilum & 1.042407 & 2894.241 & 17.69918 & 273.3682 & 67.16225 & 107.3175 & 55.7706 & 41.84747 & 22.78658 & 9.450766 & 4.053197 & 9.896028 & 1731.505 & 0.481403 & 245.462 & 0.371083 \\
\hline Galium saxatile & 9.05393 & 2008.075 & 29.98457 & 1060.031 & 52.96762 & 78.31231 & 64.80647 & 80.78113 & 42.25779 & 14.87859 & 11.11851 & 9.733144 & 2718.521 & 3.199183 & 378.6209 & 1.327421 \\
\hline Galium uliginosum & 3.672766 & 6672.83 & 351.388 & 421.7122 & 77.08589 & 187.2085 & 32.3575 & 39.82697 & 23.6526 & 21.03266 & 5.746968 & 13.12969 & 4467.982 & 1.058682 & 596.9006 & 0.79125 \\
\hline Galium verum & 3.509617 & 3735.847 & 44.50149 & 223.3004 & 83.93223 & 119.2759 & 68.10183 & 60.76437 & 30.44406 & 15.27234 & 4.527398 & 11.51421 & 2145.128 & 1.533563 & 342.9337 & 1.14702 \\
\hline Genista anglica & 8.232192 & 3068.625 & 21.4409 & 1061.224 & 69.48905 & 117.8057 & 80.79155 & 103.12 & 36.37136 & 19.50553 & 7.753936 & 6.740259 & 3616.975 & 1.352152 & 286.2508 & 0.978433 \\
\hline Genista pilosa & 8.346006 & 1545.023 & 15.56368 & 1105.198 & 44.77139 & 73.89513 & 62.73295 & 80.0138 & 40.37368 & 14.9994 & 7.712072 & 5.60138 & 2247.482 & 1.329956 & 277.7305 & 0.916127 \\
\hline Genista tinctoria & 8.758593 & 4396.686 & 61.44713 & 340.6347 & 66.09252 & 129.2364 & 57.79523 & 70.32291 & 32.27085 & 24.55221 & 3.588464 & 10.58933 & 3177.327 & 0.705323 & 250.2056 & 1.975352 \\
\hline Gentiana cruciata & 5.315932 & 1958.218 & 62.55473 & 301.0429 & 55.19481 & 69.98127 & 37.06695 & 40.6515 & 32.64927 & 12.77382 & 4.172471 & 11.30558 & 1686.251 & 1.18581 & 233.414 & 0.862868 \\
\hline Gentiana pneumonanthe & 8.159353 & 5689.491 & 40.39817 & 624.0819 & 68.23224 & 182.1934 & 68.60798 & 83.04363 & 35.79008 & 27.7682 & 4.791059 & 7.864783 & 4997.251 & 1.356047 & 258.9721 & 0.930378 \\
\hline Gentianella amarella & 1.876692 & 990.558 & 505.3345 & 2.9069 & 80.20369 & 51.8777 & 42.3596 & 10.64619 & 7.0476 & 12.32006 & 063352 & 8.249934 & 82.652 & 0.846434 & 51.2114 & 0.796616 \\
\hline
\end{tabular}



Geranium dissectu

Geranium molle

Geranium pusillum

Geranium pyrenaicum

Geranium robertianum

Geum urbanum

Glaux maritim

Glechoma hederacea

Glyceria declinata

Glyceria fluitans

Glyceria maxima

Glyceria notata

Gnaphalium luteo-album

Gnaphalium sylvaticum

Gnaphalium uliginosur

Groenlandia densa

Gymnadenia conopsea

Hammarbya paludosa

Hedera heli

Helictotrichon pratense

elictotrichon pubescens

Heracleum sphondylium

rminium monor

Herniaria glabra

Hieracium lactucella

Hieracium laevigatum

Hieracium pilosella

Hieracium sabaudum

Hieracium sect. Hieracium

Hieracium sect. Vulgata

Hieracium umbellatum

rochloe odorata

Hippophae rhamnoides

Holcus lanatus

Holcus mollis

Honckenya peploides

Hordeum murinum

Hordeum vulgar

Hottonia palustris

Humulus lupulus

Hydrocharis morsus-ranae

Hydrocotyle vulgaris

Hypericum dubium

Hypericum elodes

\begin{tabular}{|c|c|c|c|c|c|c|c|c|c|c|c|c|c|c|c|}
\hline 2.990183 & 3101.317 & 36.31332 & 458.1009 & 66.28468 & 75.03879 & 33.82895 & 32.8921 & 24.36115 & 6.850015 & 2.169591 & 11.38054 & 1506.468 & 0.474644 & 219.9581 & 1006 \\
\hline 491931 & 3621.302 & 225.248 & 481.6494 & 92.47471 & 78.8042 & 26.85145 & 30.32948 & 32.74193 & 15.16553 & 3.940076 & 10.78897 & 2983.022 & 1.306106 & 273.3437 & 0.536 \\
\hline 115296 & 4738.397 & 248.3438 & 322.7071 & 143.9572 & 130.5301 & 59.24774 & 58.21385 & 30.66816 & 14.44729 & 3.766567 & 13.99599 & 2802.599 & 1.358144 & 408.0276 & .785 \\
\hline 138636 & 72.927 & 86.58088 & 426.0483 & 115.079 & 113.3806 & 33.26782 & 40.22534 & 39.22265 & 13.42499 & 3.385243 & 16.00956 & 2727.031 & 1.955594 & 494.776 & 931 \\
\hline 70514 & 6909.4 & 69.13854 & 19.1924 & 84.47059 & 85.10015 & 33.16279 & 40.1011 & 47.16709 & 11.68571 & 4.373876 & 23.46142 & 2162.719 & 0.980332 & 253.3948 & 1.206 \\
\hline 16787 & 3076.59 & 51.18836 & 32.2273 & 62.90155 & 94.06108 & 37.56711 & 36.08149 & 27.66411 & 14.61931 & 5.286509 & 17.50164 & 3322.832 & 0.98366 & 406.6611 & 17 \\
\hline 513487 & 15.528 & 45.02096 & 719.1479 & 57.74157 & 84.92804 & 35.30528 & 33.19995 & 26.45044 & 13.36494 & 4.943237 & 16.87888 & 3049.738 & 0.917162 & 364.1761 & 11 \\
\hline 93974 & 32.892 & 535.9772 & 393.7031 & 99.54583 & 153.7639 & 40.3927 & 30.54524 & 46.85298 & 14.60606 & 5.489871 & 11.85486 & 3138.672 & 0.844619 & 273.3685 & 876 \\
\hline 90149 & 10.873 & 89.29017 & 801.6208 & 117.2852 & 139.8333 & 53.35623 & 65.29051 & 51.81058 & 24.31925 & 7.060634 & 25.63656 & 5218.443 & 1.567981 & 541.9659 & 1.472 \\
\hline 066909 & 290.29 & 270.3957 & 765.2573 & 140.1333 & 175.6175 & 50.51092 & 70.56394 & 50.38038 & 25.20058 & 11.11294 & 29.3999 & 7909.009 & 1.103301 & 853.6709 & 67 \\
\hline 197005 & 7212.52 & 237.1543 & 407.0182 & 116.0098 & 214.4505 & 44.62894 & 63.07189 & 48.59302 & 25.75426 & 8.001265 & 18.22786 & 6582.976 & 1.307889 & 565.4048 & 1.078 \\
\hline 678548 & 6614.918 & 208.9554 & 484.8013 & 109.9673 & 214.4293 & 38.05664 & 53.17953 & 34.36204 & 21.24423 & 9.652238 & 26.52625 & 5942.724 & 1.087198 & 531.5757 & \\
\hline 99705 & 93.821 & 8.6624 & 677.2017 & 102.2292 & 233.5534 & 53.1075 & 74.62158 & 58.02873 & 27.29581 & 5.701666 & 24.84615 & 7225.253 & 1.505419 & 575.4555 & \\
\hline .104105 & 49.137 & 556.1425 & 19.7667 & 142.7172 & 234.9984 & 44.52583 & 50.84599 & 42.80892 & 14.45157 & 6.932146 & 22.69044 & 2766.722 & 1.488319 & 76.3786 & 0.74 \\
\hline 798885 & 631.495 & 45.00475 & 4.2682 & 81.00793 & 110.9668 & 57.24991 & 79.83486 & 44.15787 & 16.28486 & 7.779 & 10.36272 & 3289.269 & 2.347332 & 295.7342 & 1.097 \\
\hline 425516 & 7688.649 & 240.5976 & 547.1502 & 148.1603 & 199.4432 & 61.70266 & 83.29847 & 68.97883 & 28.43224 & 7.035281 & 18.42985 & 6317.367 & 2.074532 & 599.1029 & 1.28 \\
\hline 005132 & 3951.004 & 159.9337 & 242.466 & 86.36255 & 182.0449 & 23.58461 & 25.89553 & 27.89536 & 14.30829 & 5.385005 & 9.474157 & 4575.034 & 0.979152 & 6.8999 & 0.51 \\
\hline 170204 & 403.049 & 543.7958 & 511.7179 & 4.96941 & 150.5852 & 59.20152 & 59.34944 & 47.14143 & 24.25158 & 4.433266 & 13.12192 & 5329.181 & 0.532082 & 263.2803 & 1.24 \\
\hline 34873 & 59.412 & 1.1708 & 41.8739 & 3.90574 & 211.6446 & 39.13063 & 62.05143 & 28.23809 & 20.28708 & 10.33688 & 16.72939 & 3456.867 & 2.265949 & 371.6906 & 0.71 \\
\hline 148145 & 880.48 & 33.50507 & 73.8558 & 58.79775 & 92.60026 & 35.79011 & 44.85944 & 30.23734 & 11.02041 & 10.11675 & 22.88959 & 1681.009 & 2.660703 & 66.5776 & 1 \\
\hline .091534 & 3029.413 & 26.76485 & 317.462 & 73.48883 & 88.50556 & 48.89856 & 46.49612 & 28.4909 & 8.061001 & 2.495327 & 11.71001 & 1473.268 & 0.493561 & 190.9016 & 5 \\
\hline 095009 & 54.654 & 45.22006 & 281.8191 & 110.3068 & 95.9369 & 58.32297 & 58.0713 & 29.70195 & 16.14017 & 102145 & 10.62339 & 2181.957 & 1.155565 & 15.8716 & \\
\hline .456937 & 5271.787 & 97.13095 & 716.8554 & 89.02241 & 100.4229 & 41.71834 & 47.08807 & 46.66446 & 17.09924 & 6.195194 & 25.04529 & 3219.112 & 1.367785 & 66.5072 & 20 \\
\hline 946323 & 3695.357 & 766.2478 & 171.2529 & 84.90817 & 318.7715 & 47.87387 & 46.69525 & 49.73319 & 10.26804 & 2.615698 & 9.230356 & 2255.6 & 0.700825 & 182.0123 & 19 \\
\hline 4.001901 & 3613.434 & 79.26491 & 160.9731 & 100.7423 & 119.9266 & 57.26196 & 70.39705 & 27.77744 & 14.39736 & 3.478457 & 14.0291 & 54.623 & 1.529511 & 9.0016 & \\
\hline 6.76804 & 5630.673 & 57.01855 & 297.0405 & 8.58879 & 141.6549 & 46.78042 & 52.04226 & 26.71213 & 28.83961 & 5.27963 & 9.444033 & 5438.441 & 0.493493 & 74.826 & \\
\hline 1839575 & 4027.157 & 40.11906 & 817.7162 & 79.81315 & 108.6211 & 55.61304 & 82.15546 & 46.07314 & 15.35424 & 11.63363 & 15.43587 & 2897.276 & 2.318185 & 294.3094 & .03 \\
\hline .632947 & 3585.28 & 35.07258 & 238.4453 & 85.46663 & 109.3293 & 61.27998 & 61.73522 & 31.87675 & 15.52272 & 5.481692 & 12.17167 & 2288.858 & 0.942748 & 306.5416 & \\
\hline 11.01608 & 4500.262 & 41.29833 & 918.7944 & 83.01197 & 101.4723 & 43.62805 & 58.13421 & 44.56019 & 12.25972 & 10.9259 & 28.8464 & 2306.731 & 2.180302 & 266.673 & 29 \\
\hline 9.254466 & 2586.844 & 43.43711 & 777.6828 & 52.92372 & 78.62141 & 39.68021 & 41.67237 & 30.89511 & 8.672185 & 7.238013 & 29.23509 & 1938.499 & 2.566917 & 358.6867 & Tr \\
\hline 9.847442 & 4772.903 & 51.10978 & 961.9865 & 98.50377 & 160.0213 & 81.06681 & 111.4132 & 71.70473 & 26.3636 & 10.77643 & 23.34049 & 4768.157 & 2.601879 & 357.8708 & trit \\
\hline 8.77761 & 3131.298 & 44.07767 & 344.1435 & 74.85808 & 112.7453 & 63.35361 & 72.17376 & 40.1394 & 15.4601 & 6.704996 & 12.06867 & 2229.72 & 1.433514 & 345.1808 & \\
\hline 3.794461 & 6002.183 & 119.1944 & 404.7668 & 90.30076 & 190.5722 & 39.54436 & 51.54601 & 31.62486 & 17.32394 & 8.2557 & 18.79402 & 3022.081 & 1.286965 & 581.233 & .0J \\
\hline .406563 & 4336.535 & 672.4285 & 367.5572 & 108.3268 & 263.4202 & 88.01529 & 84.60819 & 64.56658 & 17.83283 & 7.659016 & 27.97426 & 2359.247 & 1.862522 & 376.6713 & .13 \\
\hline 161333 & 8865.611 & 374.0871 & 1.2583 & 138.0408 & 210.6547 & 62.47026 & 84.09689 & 44.77264 & 31.18185 & 8.680249 & 21.1596 & 321.474 & 2.293483 & 587.6023 & 1.59 \\
\hline 979019 & 55.907 & 61.09279 & 4.9119 & 86.51654 & 122.945 & 54.62211 & 78.34681 & 45.11773 & 18.82133 & 13.77092 & 24.68948 & 3719.232 & 2.784155 & 00.5451 & 1.50 \\
\hline 1.495722 & 07.616 & 776.2731 & 13.4226 & 148.688 & 232.7916 & 59.99066 & 71.52394 & 30.94205 & 23.52944 & 3.765072 & 22.75624 & 518.038 & 1.663902 & 394.7388 & \\
\hline 3.072128 & 9.359 & 99007 & 601.9611 & .4634 & 15 & 72 & 359 & 39.02868 & 764 & 3.131458 & 19.94845 & 79.031 & 1.209668 & 2.3597 & \\
\hline 1.457322 & 18.674 & 5.4669 & 379.559 & 89.06065 & 88.50506 & 20.24805 & 8.86949 & 22.27798 & 12.41613 & 2.859716 & 7.474381 & 70.296 & 0.651998 & 6.7372 & \\
\hline .604854 & 7148.774 & 109.6638 & 983.405 & 154.1408 & 104.0398 & 30.49978 & 41.16544 & 42.57165 & 16.03091 & 2.649873 & 13.95171 & 88.163 & 3.11819 & 4.0214 & \\
\hline 193409 & 5422.391 & 140.0364 & 364.7317 & 0.0173 & 188.4006 & 29.05851 & 37.97673 & 32.19256 & 17.89285 & 10.50681 & 25.26338 & 140.357 & 1.646816 & 8.9736 & \\
\hline .838892 & 3985.063 & 84.08514 & 44112 & 9617 & 165.0345 & 43.0273 & 52.64998 & 50.6804 & 17.35281 & 9.671242 & 17.88709 & 3271.851 & 2.309405 & 68.1664 & \\
\hline .787612 & 44.654 & 159.1806 & 460.2156 & 6573 & 163.7305 & 22.71251 & 28.45519 & 24.88259 & 18.10805 & 10.71651 & 25.31157 & 943.25 & 1.26323 & 09.124 & \\
\hline .672782 & 7082.449 & 442.2958 & 467.0969 & 105.0153 & 227.6262 & 36.60947 & 9963 & 04646 & 20.97852 & 07644 & 15.37881 & 41.548 & 1.611497 & 612.1548 & \\
\hline 75457 & 86.19 & 5.5522 & 7991 & 539 & 1.8239 & 5191 & & 84 & 793 & & 596 & 916 & 9836 & 380.32 & \\
\hline 63945 & 36.25 & 97 & 1.1444 & 8772 & .3815 & 511 & 306 & 61 & 23 & 111 & 43 & & 54 & & \\
\hline
\end{tabular}




\begin{tabular}{|c|c|c|c|c|c|c|c|c|c|c|c|c|c|c|c|c|}
\hline Hypericum hirsutum & 6.330248 & 3546.519 & 38.23242 & 871.464 & 64.59505 & 61.00913 & 44.21729 & 36.15295 & 28.55545 & 9.253621 & 5.579905 & 24.66533 & 1276.567 & 1.261147 & 138.0614 & 1.614156 \\
\hline Hypericum humifusum & 6.966663 & 5405.69 & 121.719 & 765.8132 & 99.85275 & 142.395 & 66.46151 & 95.65997 & 66.60927 & 25.43699 & 7.951179 & 13.54839 & 4545.095 & 1.663333 & 365.3044 & 1.375896 \\
\hline Hypericum perforatum & 7.276227 & 4584.994 & 49.83298 & 535.8585 & 92.93256 & 126.3281 & 48.67871 & 61.10306 & 41.73053 & 21.32678 & 7.478299 & 18.03197 & 3474.749 & 1.353152 & 352.2903 & 1.790071 \\
\hline Hypericum pulchrum & 7.761789 & 3639.188 & 40.66725 & 1059.292 & 68.57378 & 122.5321 & 76.39603 & 97.54203 & 66.44763 & 21.49028 & 12.49918 & 15.50712 & 3333.087 & 3.567483 & 292.233 & 1.275044 \\
\hline Hypericum tetrapterum & 4.896114 & 8294.569 & 208.3365 & 527.2852 & 101.0741 & 253.1839 & 58.7729 & 66.81369 & 40.31836 & 26.78414 & 7.302869 & 28.0141 & 5076.364 & 1.480005 & 588.9407 & 0.931034 \\
\hline Hypochaeris radicata & 7.53174 & 4850.894 & 67.3257 & 295.7497 & 113.9157 & 144.9395 & 69.16182 & 79.48829 & 36.65762 & 22.58637 & 5.231458 & 12.82244 & 3478.171 & 1.017431 & 453.6508 & 1.705862 \\
\hline Ilex aquifolium & 5.134081 & 2144.962 & 26.39889 & 365.2651 & 50.9728 & 80.73927 & 38.99936 & 58.91824 & 30.53762 & 12.57604 & 8.596134 & 25.5057 & 1640.379 & 2.093482 & 287.8079 & 1.233919 \\
\hline Illecebrum verticillatum & 5.657404 & 5190.064 & 204.6591 & 588.3118 & 106.7895 & 161.3264 & 77.68891 & 122.7785 & 61.93445 & 24.3205 & 7.437156 & 11.27645 & 5308.603 & 1.664911 & 372.2755 & 1.029796 \\
\hline Impatiens noli-tangere & 4.824919 & 5473.326 & 149.6775 & 530.4205 & 90.10259 & 186.9181 & 54.80987 & 65.94338 & 51.83133 & 22.56408 & 7.783677 & 16.94568 & 3829.72 & 1.30234 & 272.1924 & 1.283442 \\
\hline Inula britannica & 2.098608 & 8188.223 & 262.4311 & 564.1056 & 130.0924 & 137.5073 & 40.59457 & 49.58056 & 27.03828 & 23.06168 & 6.376382 & 15.32211 & 8251.537 & 0.793026 & 1007.748 & 0.575084 \\
\hline Inula conyzae & 7.150783 & 3560.688 & 57.20281 & 551.7196 & 83.29379 & 119.7682 & 55.95075 & 47.2369 & 43.47304 & 12.96727 & 6.809256 & 33.63574 & 2312.068 & 1.345291 & 312.1404 & 1.117921 \\
\hline Iris pseudacorus & 5.415559 & 8112.205 & 205.2399 & 456.0517 & 132.9453 & 257.6129 & 63.65294 & 85.67792 & 46.69551 & 28.11105 & 10.18682 & 25.53604 & 6470.663 & 1.311404 & 649.222 & 1.110656 \\
\hline Isolepis setacea & 4.304259 & 7488.845 & 405.0316 & 427.83 & 121.1272 & 200.4216 & 38.74962 & 44.18451 & 37.62806 & 19.13308 & 4.158853 & 14.88835 & 5046.827 & 0.687375 & 413.0055 & 1.149635 \\
\hline Jasione montana & 5.940461 & 2666.711 & 25.39773 & 208.3357 & 60.98872 & 88.95469 & 59.05139 & 57.58729 & 31.79128 & 13.07043 & 3.305481 & 9.295957 & 1856.09 & 0.61087 & 290.3474 & 1.02506 \\
\hline Juncus acutiflorus & 5.638133 & 7737.022 & 129.7036 & 412.8652 & 90.81496 & 206.2463 & 41.41714 & 61.50074 & 34.65807 & 20.46767 & 6.208989 & 13.40392 & 3803.188 & 1.243461 & 602.9539 & 0.936955 \\
\hline Juncus alpinoarticulatus s. atricapillus & 4.293779 & 6577.081 & 607.8522 & 315.4781 & 110.9363 & 229.9851 & 39.11858 & 75.57247 & 34.33664 & 16.81626 & 4.202334 & 11.99601 & 4327.638 & 0.975837 & 284.9977 & 1.392438 \\
\hline Juncus ambiguus & 2.720062 & 8007.584 & 612.0069 & 499.443 & 134.1561 & 246.9995 & 54.22273 & 48.72486 & 54.31956 & 22.62237 & 5.711659 & 21.35721 & 4171.042 & 1.472327 & 324.885 & 0.819508 \\
\hline Juncus articulatus & 4.475034 & 6775.37 & 513.2687 & 439.2212 & 125.9501 & 204.363 & 39.93625 & 52.73307 & 41.71939 & 24.3109 & 5.359728 & 16.34128 & 7476.276 & 1.087526 & 767.5236 & 1.466198 \\
\hline Juncus balticus & 6.259968 & 7452.676 & 630.2971 & 408.8947 & 110.1052 & 261.7425 & 38.38092 & 76.6249 & 29.52652 & 26.4772 & 3.968138 & 10.81735 & 4533.739 & 1.380364 & 339.8239 & 1.86501 \\
\hline Juncus bufonius & 5.39846 & 9001.805 & 685.7204 & 551.014 & 159.2134 & 242.5156 & 50.86399 & 65.16776 & 61.4556 & 26.19805 & 7.417151 & 19.506 & 6243.106 & 1.987507 & 539.1104 & 1.876345 \\
\hline Juncus bufonius ag. (incl. J. ambiguus) & 4.22776 & 8257.021 & 658.1892 & 541.8225 & 147.5967 & 264.6379 & 39.60495 & 48.26259 & 37.5163 & 24.32584 & 6.858011 & 17.56998 & 6554.118 & 1.031998 & 712.2318 & 1.112725 \\
\hline Juncus bulbosus & 10.2861 & 6038.53 & 232.5147 & 541.3087 & 91.21657 & 255.5274 & 22.28877 & 31.37268 & 22.24366 & 21.72629 & 8.002158 & 13.07331 & 4697.991 & 0.915542 & 558.5478 & 1.170682 \\
\hline Juncus compressus & 7.294968 & 8120.034 & 291.7409 & 445.7331 & 118.6154 & 234.7377 & 33.98773 & 43.46147 & 33.35013 & 26.52793 & 4.891505 & 25.14193 & 7735.687 & 0.958046 & 588.0513 & 1.767307 \\
\hline Juncus conglomeratus & 6.154888 & 8291.049 & 123.0335 & 521.1878 & 92.48186 & 227.7112 & 58.12667 & 75.70862 & 33.75361 & 25.99075 & 7.085801 & 15.81923 & 4578.967 & 1.865398 & 485.1275 & 1.017634 \\
\hline Juncus conglomeratus $x$ effusus & 5.916714 & 7214.653 & 97.84597 & 338.1684 & 94.33997 & 119.0621 & 29.21866 & 37.70245 & 23.10315 & 15.76953 & 5.527261 & 11.81881 & 2537.166 & 0.911105 & 386.6684 & 1.019359 \\
\hline Juncus effusus & 7.470487 & 9139.078 & 176.895 & 583.5115 & 142.8727 & 267.0276 & 75.44659 & 111.1645 & 54.20945 & 32.14857 & 11.56116 & 30.03024 & 5719.704 & 2.266715 & 744.1073 & 1.249572 \\
\hline Juncus filiformis & 4.979304 & 6599.751 & 112.7377 & 324.653 & 129.7548 & 165.0086 & 30.38831 & 34.16819 & 27.10532 & 16.97919 & 7.015331 & 10.7565 & 780.757 & 1.346504 & 709.1015 & 2.099901 \\
\hline Juncus gerardi & 3.104848 & 6062.979 & 592.8754 & 394.6137 & 101.6581 & 159.1855 & 36.79192 & 31.39082 & 42.8686 & 14.91984 & 5.41709 & 12.4908 & 3838.805 & 1.016022 & 286.461 & 0.883093 \\
\hline Juncus inflexus & 5.187783 & 6450.096 & 393.4968 & 529.3931 & 111.3181 & 181.2673 & 43.47576 & 49.71624 & 45.88532 & 25.57155 & 6.271594 & 21.14241 & 6452.949 & 1.258847 & 699.0945 & 1.272897 \\
\hline Juncus maritimus & 5.016539 & 5438.283 & 603.6811 & 327.0564 & 136.2432 & 214.2266 & 43.23386 & 36.15204 & 37.15345 & 15.87405 & 5.758134 & 11.79502 & 3968.224 & 0.712525 & 229.3768 & 1.027423 \\
\hline Juncus squarrosus & 9.038535 & 3569.543 & 18.70813 & 833.5603 & 73.19743 & 128.1244 & 93.19604 & 132.694 & 51.87366 & 20.51339 & 6.003393 & 7.353987 & 4004.899 & 1.949355 & 351.8902 & 0.935258 \\
\hline Juncus subnodulosus & 5.909981 & 7794.014 & 177.3771 & 433.616 & 134.8714 & 191.4434 & 34.36189 & 51.56863 & 22.82618 & 23.13869 & 10.58067 & 29.74683 & 4495.971 & 1.817309 & 775.2599 & 1.061954 \\
\hline Juncus tenageia & 5.540809 & 8476.644 & 498.2091 & 726.4959 & 111.6411 & 199.1473 & 39.95434 & 56.28313 & 37.93689 & 13.86644 & 5.3147 & 11.3531 & 3318.955 & 0.482853 & 357.8685 & 1.179187 \\
\hline Juniperus communis & 6.95192 & 1522.354 & 26.81575 & 683.0512 & 46.35881 & 75.55717 & 57.51819 & 69.9541 & 45.98126 & 13.77533 & 10.20436 & 8.75893 & 2425.398 & 3.180419 & 444.7885 & 1.045901 \\
\hline Kickxia elatine & 3.554834 & 2688.516 & 69.57151 & 841.1914 & 121.8457 & 72.51546 & 26.85614 & 26.99085 & 27.62593 & 11.21146 & 2.655068 & 10.07523 & 1831.49 & 1.734152 & 209.8931 & 0.519458 \\
\hline Knautia arvensis & 2.207944 & 4122.064 & 48.46315 & 420.972 & 83.4299 & 88.05631 & 36.58042 & 39.32786 & 33.5642 & 14.31063 & 3.742253 & 11.78052 & 2678.646 & 0.613619 & 333.681 & 0.561369 \\
\hline Koeleria macrantha & 2.013727 & 2415.024 & 37.7449 & 179.0371 & 49.81423 & 92.05822 & 53.96141 & 49.44902 & 26.72789 & 12.27212 & 4.780498 & 9.867817 & 1603.388 & 1.351958 & 264.5166 & 1.138989 \\
\hline Koeleria pyramidata & 1.398409 & 3396.811 & 21.32034 & 236.0471 & 72.62137 & 152.0875 & 73.39281 & 43.09565 & 18.74985 & 9.814128 & 2.790594 & 9.319244 & 1492.188 & 0.506644 & 168.9963 & 0.293084 \\
\hline Lactuca serriola & 5.63192 & 4847.57 & 424.6203 & 880.0324 & 144.6121 & 136.1906 & 42.37939 & 52.3403 & 56.60292 & 19.92763 & 5.896254 & 28.7507 & 4434.576 & 2.123868 & 467.7684 & 0.820851 \\
\hline Lamiastrum galeobdolon & 3.707776 & 2921.618 & 37.84123 & 291.0602 & 53.97501 & 83.51432 & 36.12692 & 32.21153 & 24.29724 & 11.7454 & 6.990302 & 17.11188 & 1824.117 & 1.870426 & 144.285 & 0.873535 \\
\hline Lamium album & 6.463295 & 3778.619 & 61.29343 & 758.0534 & 88.66351 & 84.75974 & 32.66574 & 37.91658 & 22.9146 & 12.13081 & 7.171864 & 21.97749 & 2960.763 & 1.348668 & 341.1983 & 1.308373 \\
\hline Lamium amplexicaule & 2.420968 & 3753.447 & 111.1457 & 841.3964 & 133.607 & 102.7602 & 27.14397 & 33.58119 & 37.9773 & 10.69893 & 2.971606 & 14.39378 & 2027.091 & 1.952021 & 333.1247 & 0.696484 \\
\hline Lamium maculatum & 5.644373 & 3486.761 & 60.61795 & 743.2425 & 68.09663 & 79.64866 & 37.94314 & 34.67455 & 23.16555 & 10.85899 & 6.290793 & 18.42199 & 1981.858 & 1.012196 & 214.9984 & 1.22587 \\
\hline Lamium purpureum & 4.877318 & 4049.483 & 93.1368 & 721.5446 & 131.1238 & 99.59564 & 33.28929 & 39.63286 & 41.021 & 14.83049 & 4.312823 & 18.45947 & 3301.222 & 1.675394 & 480.0272 & 0.925886 \\
\hline Lapsana communis & 6.99007 & 3895.122 & 70.80427 & 683.6807 & 90.29847 & 98.50603 & 39.72248 & 47.13045 & 34.83387 & 16.13072 & 9.002515 & 23.95783 & 2792.68 & 1.994567 & 205.7451 & 1.53266 \\
\hline Lathyrus linifolius & 4.643766 & 2255.572 & 35.15436 & 685.6945 & 52.32888 & 79.3509 & 39.10209 & 49.08195 & 53.72488 & 9.353259 & 11.14504 & 15.24403 & 1723.337 & 3.631908 & 268.3327 & 17829 \\
\hline
\end{tabular}




\begin{tabular}{|c|c|c|c|c|c|c|c|c|c|c|c|c|c|c|c|c|}
\hline Lathyrus palustris & 3.713729 & 5161.691 & 126.177 & 429.165 & 100.2253 & 193.5648 & 33.19763 & 41.49973 & 24.41013 & 18.49799 & 8.890242 & 17.7498 & 3085.71 & 1.282742 & 642.6744 & 0.563706 \\
\hline Lathyrus pratensis & 3.340695 & 7498.688 & 145.298 & 417.3504 & 120.7026 & 129.7822 & 40.43345 & 48.96555 & 33.35402 & 28.88138 & 4.518084 & 11.42871 & 5529.414 & 0.683864 & 513.249 & 0.738875 \\
\hline Lathyrus tuberosus & 3.683364 & 4017.771 & 100.315 & 592.8439 & 109.1082 & 82.67175 & 27.85086 & 32.03568 & 35.6502 & 17.49418 & 4.911305 & 22.36415 & 2766.787 & 1.963928 & 351.0282 & 0.512135 \\
\hline Legousia speculum-veneris & 2.753713 & 2577.096 & 81.6735 & 507.0938 & 75.31121 & 75.03779 & 25.03161 & 25.9303 & 48.54855 & 11.91392 & 2.79412 & 9.270793 & 1930.635 & 2.898306 & 220.2074 & 0.419954 \\
\hline Lemna gibba & 4.194445 & 5085.307 & 225.279 & 370.0192 & 103.3284 & 190.7939 & 31.59003 & 38.87057 & 30.40236 & 21.32014 & 8.538701 & 26.08859 & 5396.738 & 1.703634 & 552.2312 & 1.177242 \\
\hline Lemna gibba + L. minor & 4.736669 & 7017.649 & 237.8423 & 426.3392 & 112.0319 & 186.0844 & 25.68738 & 37.87287 & 28.43809 & 21.61746 & 9.465296 & 25.90862 & 5444.085 & 1.461015 & 490.0492 & 1.036551 \\
\hline Lemna minor & 5.022608 & 6433.867 & 192.556 & 426.6297 & 108.1813 & 192.4191 & 30.38342 & 40.26645 & 31.23438 & 20.53896 & 10.63876 & 26.29391 & 5038.754 & 1.524033 & 520.3918 & 1.063884 \\
\hline Lemna trisulca & 4.100772 & 4907.91 & 203.0597 & 504.8499 & 90.28728 & 179.7243 & 23.86954 & 29.90415 & 28.20009 & 18.61057 & 9.963694 & 25.36219 & 4848.182 & 1.194357 & 493.0218 & 0.888352 \\
\hline Leontodon autumnalis & 4.613974 & 8789.06 & 703.6129 & 400.3382 & 177.6999 & 174.6458 & 48.46839 & 59.6027 & 34.22256 & 22.57392 & 5.589082 & 13.4588 & 5920.031 & 1.02317 & 636.5434 & 1.444021 \\
\hline Leontodon hispidus & 2.742936 & 3767.658 & 54.08086 & 450.1051 & 77.85208 & 83.56386 & 39.30979 & 39.7422 & 23.66486 & 11.81223 & 3.157392 & 10.5926 & 2833.295 & 0.590137 & 292.9902 & 0.606978 \\
\hline Leontodon saxatilis & 4.415389 & 7672.13 & 700.1959 & 265.3563 & 141.8301 & 257.2001 & 91.57188 & 90.53594 & 49.61812 & 28.93338 & 4.958722 & 13.31346 & 3582.897 & 0.994735 & 435.7967 & 1.508938 \\
\hline Lepidium draba & 2.143442 & 4490.972 & 639.5657 & 457.4199 & 159.3801 & 112.1223 & 41.68418 & 46.34566 & 41.44887 & 20.68068 & 4.178705 & 22.96958 & 3200.586 & 1.685588 & 632.7486 & 1.08265 \\
\hline Leucanthemum vulgare & 3.725566 & 4972.494 & 66.74266 & 396.5328 & 89.41841 & 105.2184 & 39.87735 & 45.63714 & 27.27695 & 18.55592 & 4.157442 & 12.05983 & 3794.245 & 0.751081 & 320.0048 & 1.146679 \\
\hline Leucojum aestivum & 3.103168 & 7457.311 & 136.3774 & 889.9877 & 100.023 & 175.2037 & 39.76911 & 52.71399 & 37.51268 & 23.25793 & 6.174384 & 22.04895 & 4036.285 & 0.946629 & 251.6859 & 0.780639 \\
\hline Leymus arenarius & 1.818335 & 3735.5 & 765.3797 & 266.7331 & 102.1025 & 148.844 & 67.26344 & 80.47984 & 42.31517 & 17.36973 & 3.862463 & 26.63236 & 2421.672 & 1.965931 & 403.6986 & 0.777472 \\
\hline Ligustrum vulgare & 5.567401 & 2601.739 & 210.8556 & 545.0192 & 77.16553 & 94.85189 & 36.55596 & 41.08676 & 39.33346 & 13.17971 & 5.575243 & 26.90431 & 2145.411 & 0.952525 & 332.4696 & 1.35055 \\
\hline Limonium vulgare & 2.380214 & 7781.911 & 494.0556 & 276.1221 & 160.0106 & 302.4707 & 42.21869 & 49.50925 & 57.56354 & 18.77309 & 5.105535 & 31.96144 & 2255.005 & 0.369965 & 303.4916 & 0.755996 \\
\hline Linaria vulgaris & 6.113371 & 4042.579 & 82.39908 & 449.1101 & 101.4887 & 107.4031 & 46.51797 & 52.54618 & 35.2669 & 18.46076 & 6.431637 & 17.40137 & 3154.443 & 1.965174 & 408.3865 & 1.471949 \\
\hline Linum catharticum & 3.878286 & 6240.435 & 587.0236 & 418.9905 & 103.2462 & 263.841 & 70.01707 & 70.72672 & 45.28339 & 23.75261 & 5.124982 & 11.54926 & 4190.719 & 0.617244 & 304.4471 & 1.161684 \\
\hline Liparis loeselii & 4.430297 & 5265.216 & 405.7722 & 473.1057 & 77.95746 & 176.1307 & 24.80687 & 42.50966 & 19.28814 & 18.88959 & 8.444643 & 13.31328 & 5880.155 & 1.811051 & 890.2705 & 1.230772 \\
\hline Listera ovata & 5.568666 & 3382.879 & 45.97324 & 772.5587 & 58.04551 & 116.0539 & 54.056 & 48.38639 & 49.06623 & 18.97262 & 5.898802 & 26.96986 & 3152.698 & 1.661299 & 297.925 & 1.540388 \\
\hline Lithospermum officinale & 4.658246 & 3360.76 & 53.22439 & 527.1599 & 74.20224 & 104.6039 & 37.33011 & 41.30947 & 36.99342 & 12.59095 & 4.627431 & 25.02769 & 2109.229 & 1.815062 & 290.1766 & 0.806119 \\
\hline Littorella uniflora & 6.085464 & 6394.138 & 455.9441 & 540.733 & 99.41683 & 212.0277 & 14.91493 & 27.81687 & 15.58771 & 13.636 & 5.076863 & 14.48833 & 3607.114 & 0.772911 & 356.9436 & 1.288261 \\
\hline Lolium multiflorum & 4.957084 & 6367.763 & 124.4652 & 562.3399 & 123.9595 & 106.0373 & 33.72471 & 47.94168 & 47.51863 & 22.87441 & 5.569765 & 14.72938 & 4550.313 & 1.989333 & 405.2503 & 0.955478 \\
\hline Lolium perenne & 3.290379 & 5108.498 & 586.1911 & 514.8692 & 117.7627 & 105.1008 & 31.78021 & 39.68733 & 34.15808 & 16.76363 & 4.557054 & 11.0906 & 4060.371 & 1.046067 & 429.3898 & 1.050343 \\
\hline Lonicera periclymenum & 6.790628 & 3390.637 & 50.99499 & 709.1404 & 72.64165 & 127.8324 & 52.64448 & 75.39115 & 42.72565 & 17.19714 & 10.09711 & 27.37288 & 3700.911 & 2.600643 & 452.5245 & 1.524355 \\
\hline Lotus corniculatus ag. (incl. L. glaber) & 4.085049 & 6802.642 & 694.3585 & 312.0066 & 142.4492 & 154.1974 & 75.66022 & 76.45576 & 42.1681 & 23.26401 & 4.860073 & 14.72768 & 3691.967 & 1.046605 & 483.0963 & 1.399584 \\
\hline Lotus corniculatus $\mathrm{v}$. corniculatus & 4.72267 & 5098.765 & 672.941 & 306.3649 & 114.5173 & 158.9412 & 80.64047 & 79.82626 & 44.18865 & 22.04357 & 4.681857 & 12.13322 & 3274.274 & 0.912488 & 374.8091 & 1.416537 \\
\hline Lotus glaber & 3.273646 & 6263.182 & 605.7836 & 407.8761 & 128.9376 & 190.8156 & 39.70263 & 40.35634 & 39.31825 & 20.11142 & 5.108126 & 13.78449 & 3490.044 & 1.029173 & 316.0023 & 0.99615 \\
\hline Lotus pedunculatus & 3.99955 & 6900.102 & 266.4831 & 426.1892 & 92.52302 & 195.1506 & 37.46767 & 46.83454 & 30.07977 & 22.62414 & 5.891852 & 13.87656 & 4521.341 & 1.03818 & 523.6701 & 0.751511 \\
\hline Luzula campestris & 8.55363 & 4550.745 & 52.8278 & 296.6127 & 94.43336 & 140.0294 & 79.91531 & 85.72658 & 43.71506 & 22.28022 & 6.926793 & 11.70867 & 3061.751 & 1.485444 & 375.9862 & 1.899536 \\
\hline Luzula multiflora & 7.316192 & 8480.981 & 110.5645 & 691.7588 & 100.4229 & 260.822 & 88.09427 & 123.7338 & 39.30011 & 36.92018 & 5.932788 & 11.14692 & 6170.041 & 2.029008 & 437.0524 & 1.232497 \\
\hline Luzula multiflora s. congesta & 6.223716 & 9720.499 & 54.3387 & 567.3269 & 84.97741 & 235.6402 & 65.76731 & 88.03092 & 33.90343 & 28.50601 & 6.163682 & 10.11075 & 5124.9 & 0.968387 & 390.9578 & 0.960685 \\
\hline Luzula multiflora s. multiflora & 7.009646 & 7213.21 & 61.33593 & 792.5612 & 85.54537 & 226.5702 & 76.32163 & 105.7403 & 54.21107 & 31.07746 & 9.055165 & 21.04736 & 5265.702 & 3.154346 & 368.4181 & 1.298668 \\
\hline Luzula pilosa & 4.141929 & 2761.277 & 34.10768 & 377.138 & 57.99877 & 88.5577 & 32.70451 & 49.23242 & 23.90052 & 12.31315 & 8.98888 & 33.38464 & 1530.105 & 2.528513 & 213.6624 & 1.128873 \\
\hline Lychnis flos-cuculi & 4.38003 & 4945.166 & 198.1793 & 424.9255 & 102.9139 & 150.3902 & 27.74578 & 34.69096 & 25.94426 & 19.1943 & 5.804264 & 15.90887 & 3481.822 & 0.95057 & 416.8688 & 0.711731 \\
\hline Lycopodiella inundata & 8.103737 & 3904.425 & 14.5683 & 961.5161 & 63.94556 & 146.0637 & 63.86448 & 91.59326 & 34.93669 & 22.70882 & 5.403579 & 6.203025 & 3553.128 & 1.11225 & 201.1474 & 0.706447 \\
\hline Lycopodium clavatum & 8.596321 & 1485.382 & 16.23086 & 1044.776 & 43.99681 & 77.01274 & 64.64093 & 79.04314 & 34.58046 & 14.02034 & 6.713837 & 5.81564 & 2005.54 & 2.578183 & 358.831 & 1.028712 \\
\hline Lycopus europaeus & 4.984279 & 7063.715 & 247.1073 & 534.8984 & 122.8037 & 243.8949 & 54.43971 & 77.46945 & 44.37018 & 23.81188 & 10.40117 & 28.23865 & 5721.273 & 1.45773 & 759.8173 & 1.559512 \\
\hline Lysimachia nemorum & 4.515546 & 5342.207 & 60.39608 & 625.6654 & 70.90264 & 158.5851 & 47.47365 & 50.29177 & 50.75889 & 21.73992 & 5.992419 & 19.52593 & 3287.664 & 2.160489 & 187.4306 & 0.848564 \\
\hline Lysimachia nummularia & 6.279715 & 7910.141 & 192.1818 & 545.7233 & 131.2486 & 191.0118 & 54.89305 & 68.96016 & 51.29381 & 27.27103 & 7.136389 & 25.30901 & 6351.793 & 1.087699 & 632.7975 & 1.180868 \\
\hline Lysimachia thyrsiflora & 5.688706 & 8103.267 & 169.3442 & 442.0059 & 141.099 & 185.434 & 50.13703 & 70.41957 & 34.14183 & 25.81245 & 8.874706 & 33.08458 & 5556.749 & 1.536396 & 601.4437 & 0.977053 \\
\hline Lysimachia vulgaris & 5.88183 & 8391.78 & 128.6944 & 519.7133 & 104.7326 & 283.9193 & 73.07896 & 102.3336 & 53.4043 & 29.39596 & 10.23193 & 30.36668 & 4985.017 & 2.114173 & 660.4201 & 1.046009 \\
\hline Lythrum portula & 4.597232 & 7597.912 & 227.1653 & 446.0493 & 125.8046 & 239.5935 & 33.28876 & 49.47071 & 34.06619 & 20.88721 & 5.350342 & 18.44796 & 7504.556 & 0.832968 & 914.3384 & 0.912968 \\
\hline Lythrum salicaria & 4.941909 & 7165.752 & 229.8603 & 499.1365 & 115.6332 & 221.6143 & 50.13815 & 72.47492 & 45.51697 & 24.74603 & 9.431885 & 29.50502 & 5455.422 & 1.697613 & 747.0847 & 1.114583 \\
\hline Maianthemum bifolium & 4.209062 & 2577.569 & 31.25192 & 367.0378 & 55.64132 & 4.52741 & 34.91134 & 57.76743 & 73328 & 0.6775 & 35541 & 27.24694 & 66.791 & 2.343741 & 5.8364 & 2 \\
\hline
\end{tabular}




\begin{tabular}{|c|c|c|c|c|c|c|c|c|c|c|c|c|c|c|c|c|}
\hline Malus sylvestris & 10.02302 & 4302.149 & 62.0828 & 820.7679 & 100.3127 & 128.9129 & 56.32181 & 70.8934 & 38.34202 & 14.57328 & 15.86337 & 31.17259 & 2854.822 & 3.184908 & 367.2068 & 2.744725 \\
\hline Malva moschata & 4.028614 & 5714.62 & 84.67851 & 513.368 & 118.3146 & 94.4336 & 28.80898 & 34.06379 & 41.71238 & 20.82332 & 5.387211 & 21.70759 & 4763.112 & 1.285462 & 506.0631 & 1.693255 \\
\hline Malva neglecta & 3.742658 & 2659.949 & 126.5833 & 825.5198 & 91.59451 & 80.11326 & 26.53351 & 29.05053 & 34.09782 & 10.52833 & 3.290571 & 18.44639 & 2614.917 & 1.126505 & 378.319 & 1.046112 \\
\hline Malva sylvestris & 2.983968 & 3369.841 & 81.70505 & 629.7691 & 110.9702 & 102.5755 & 27.93504 & 32.89183 & 41.2056 & 11.91708 & 3.850732 & 20.64441 & 2698.971 & 1.378201 & 543.2405 & 1.007797 \\
\hline Matricaria discoidea & 2.863097 & 4973.681 & 527.0606 & 563.3412 & 144.3759 & 119.6931 & 31.81622 & 40.71254 & 42.13973 & 16.33048 & 4.462585 & 12.82905 & 3828.957 & 1.399574 & 434.1972 & 1.059299 \\
\hline Matricaria recutita & 3.387603 & 4268.484 & 515.7061 & 593.7238 & 139.2636 & 112.7153 & 29.84986 & 37.91271 & 44.67718 & 16.00092 & 3.896557 & 14.23632 & 3204.291 & 2.939666 & 402.9151 & 1.249621 \\
\hline Medicago arabica & 1.491795 & 3549.385 & 658.5063 & 393.2397 & 109.0868 & 106.5486 & 28.0526 & 30.42553 & 19.83442 & 11.0949 & 2.631481 & 10.50004 & 2457.325 & 1.418158 & 332.6632 & 0.654353 \\
\hline Medicago falcata & 1.401123 & 3742.169 & 57.43753 & 276.3073 & 86.74139 & 74.99522 & 33.38972 & 37.20679 & 27.40901 & 11.14935 & 3.232697 & 9.179874 & 2218.287 & 1.2344 & 295.973 & 0.399575 \\
\hline Medicago falcata + M. sativa & 1.401311 & 2815.756 & 42.14964 & 448.5343 & 80.91442 & 71.03801 & 37.30476 & 35.85994 & 23.21272 & 10.30785 & 4.637336 & 9.277737 & 2674.865 & 0.904851 & 381.3918 & 0.732463 \\
\hline Medicago lupulina & 2.896476 & 4524.045 & 404.6389 & 434.4207 & 113.0972 & 100.5684 & 39.59237 & 41.62895 & 25.04934 & 16.2611 & 4.526748 & 10.77578 & 2974.106 & 0.933244 & 367.8613 & 0.557319 \\
\hline Medicago sativa & 3.667467 & 3614.506 & 85.98748 & 535.807 & 101.7482 & 82.02814 & 34.53107 & 35.68346 & 30.44784 & 11.96691 & 5.094659 & 12.27335 & 2759.619 & 0.592104 & 299.7721 & 0.786359 \\
\hline Melampyrum pratense & 8.556671 & 2523.096 & 40.23113 & 688.5711 & 65.93721 & 96.29005 & 62.78284 & 81.63229 & 39.19144 & 15.13426 & 11.08954 & 20.7152 & 2274.63 & 2.617583 & 299.0446 & 1.371479 \\
\hline Melica uniflora & 3.44487 & 2295.746 & 35.64643 & 265.3352 & 43.02921 & 60.66927 & 24.20388 & 20.0815 & 24.07202 & 8.189604 & 4.467861 & 15.72982 & 980.8821 & 1.158001 & 134.4829 & 0.838429 \\
\hline Melilotus albus & 2.989464 & 4945.985 & 412.9599 & 419.2097 & 151.3191 & 126.7205 & 34.81729 & 40.09971 & 40.34503 & 15.23953 & 3.189878 & 17.76015 & 2971.151 & 1.82399 & 394.0341 & 0.717799 \\
\hline Melilotus altissimus & 3.166297 & 3058.267 & 609.8939 & 545.9939 & 84.68768 & 81.63084 & 42.82612 & 44.63861 & 35.94872 & 14.23885 & 5.799097 & 16.71445 & 2642.934 & 0.455133 & 227.4402 & 0.619448 \\
\hline Melilotus officinalis & 3.275214 & 4577.785 & 245.0591 & 455.0477 & 127.6937 & 129.6151 & 44.68365 & 48.17314 & 45.49385 & 15.95494 & 2.714688 & 16.74048 & 2914.093 & 1.685167 & 421.0582 & 0.760657 \\
\hline Mentha aquatica & 4.36322 & 6838.036 & 368.4699 & 541.6731 & 119.8794 & 212.6265 & 40.96588 & 61.73006 & 40.77188 & 26.6966 & 9.037678 & 30.39275 & 7624.364 & 1.388638 & 858.4317 & 1.189065 \\
\hline Mentha arvensis & 4.63534 & 8292.851 & 276.2566 & 439.2014 & 136.7754 & 183.4564 & 54.47883 & 70.89006 & 55.53832 & 37.79247 & 8.651061 & 18.13579 & 6846.211 & 1.999852 & 531.3827 & 1.158954 \\
\hline Mentha x verticillata & 4.317549 & 3985.44 & 103.8085 & 367.2771 & 86.54743 & 140.9437 & 29.9902 & 38.5492 & 37.925 & 24.26625 & 5.32146 & 25.17075 & 7022.171 & 0.85189 & 567.4437 & 0.953023 \\
\hline Menyanthes trifoliata & 5.350155 & 5881.824 & 151.2613 & 412.0959 & 121.7604 & 164.3598 & 27.34426 & 44.75732 & 25.13958 & 20.89955 & 9.2857 & 29.66104 & 3762.047 & 1.957343 & 573.6277 & 0.812837 \\
\hline Mercurialis annua & 4.012722 & 2837.337 & 89.69963 & 904.4297 & 95.79596 & 77.81483 & 30.78025 & 34.55609 & 46.04812 & 13.5827 & 3.414302 & 18.12782 & 2438.353 & 2.272752 & 364.7423 & 0.933552 \\
\hline Mercurialis perennis & 3.37096 & 2787.298 & 34.99359 & 529.9638 & 48.48904 & 76.24516 & 33.72159 & 31.62737 & 27.0778 & 12.52299 & 4.445903 & 16.03365 & 1815.394 & 0.978373 & 239.0413 & 0.871298 \\
\hline Mespilus germanica & 5.322612 & 2782.728 & 26.83968 & 468.6058 & 54.57659 & 92.05202 & 43.17209 & 50.80579 & 25.83115 & 9.872061 & 9.740273 & 25.79218 & 1362.636 & 2.699762 & 156.5727 & 1.380763 \\
\hline Milium effusum & 4.417634 & 2981.216 & 36.42469 & 318.2892 & 59.50434 & 89.76519 & 32.66425 & 37.26307 & 26.59534 & 11.25692 & 9.75115 & 17.78251 & 1494.518 & 2.831562 & 183.1389 & 1.266065 \\
\hline Milium vernale & 1.100435 & 2492.772 & 40.2162 & 161.6768 & 72.84884 & 91.97395 & 59.49359 & 48.60709 & 39.19775 & 10.55802 & 3.828339 & 10.06639 & 1673.439 & 1.935621 & 258.8818 & 0.759094 \\
\hline Minuartia hybrida & 1.005515 & 3497.468 & 39.78469 & 201.1678 & 140.397 & 99.74566 & 68.821 & 53.43865 & 54.36067 & 11.54047 & 3.075011 & 9.703475 & 1644.853 & 0.578829 & 359.8995 & 0.58194 \\
\hline Moehringia trinervia & 6.398678 & 3273.173 & 46.21098 & 745.4585 & 69.23863 & 113.6245 & 42.05499 & 60.74971 & 36.19434 & 16.66674 & 7.560212 & 27.52878 & 3188.548 & 1.869243 & 400.6791 & 1.644053 \\
\hline Molinia caerulea & 9.40983 & 4393.313 & 56.63775 & 850.1296 & 80.86976 & 216.0335 & 110.7081 & 164.0498 & 66.38139 & 33.32554 & 11.08967 & 12.4246 & 5707.799 & 3.700715 & 474.1416 & 1.35138 \\
\hline Mycelis muralis & 5.023043 & 2965.634 & 46.32765 & 565.6298 & 55.99823 & 84.84163 & 42.09197 & 38.53369 & 31.99658 & 11.6271 & 5.037515 & 20.49295 & 2233.869 & 1.194773 & 268.3809 & 1.194117 \\
\hline Myosotis arvensis & 5.524088 & 4446.344 & 84.40746 & 632.9564 & 113.9944 & 108.1951 & 45.43324 & 54.10378 & 43.7808 & 15.08231 & 4.278014 & 18.96643 & 2955.296 & 2.628761 & 477.2852 & 1.023288 \\
\hline Myosotis discolor & 3.260791 & 9654.789 & 83.3441 & 494.9565 & 133.3397 & 221.1357 & 57.09524 & 71.349 & 52.22984 & 41.73179 & 6.277406 & 12.04302 & 6629.111 & 3.708716 & 533.246 & 0.900275 \\
\hline Myosotis laxa + M. scorpioides & 5.829819 & 6791.435 & 344.0545 & 503.3958 & 115.374 & 243.7792 & 46.4059 & 67.3213 & 38.391 & 26.75486 & 9.146153 & 28.40074 & 6898.892 & 1.311112 & 624.9199 & 1.221376 \\
\hline Myosotis laxa s. cespitosa & 4.087841 & 6792.909 & 365.4267 & 438.7736 & 114.6161 & 209.9593 & 42.35611 & 60.57347 & 44.00897 & 26.29664 & 6.660749 & 24.7043 & 6261.643 & 1.196129 & 550.3874 & 1.158036 \\
\hline Myosotis ramosissima & 2.389071 & 2594.501 & 56.9367 & 186.2868 & 56.92493 & 100.2494 & 61.09172 & 54.41335 & 31.03293 & 12.12452 & 4.856982 & 15.18586 & 1606.453 & 1.566231 & 268.4751 & 0.792217 \\
\hline Myosotis scorpioides & 4.009904 & 5435.868 & 190.8638 & 455.4575 & 100.2939 & 182.1225 & 30.08695 & 44.13814 & 32.27624 & 21.11573 & 6.267766 & 28.6789 & 6026.791 & 1.058134 & 471.4248 & 0.882781 \\
\hline Myosotis stricta & 3.789565 & 3054.33 & 43.14411 & 222.0427 & 68.42792 & 106.406 & 63.44021 & 61.60318 & 41.24023 & 13.24092 & 4.062939 & 16.98677 & 2058.757 & 3.411732 & 348.3447 & 1.501771 \\
\hline Myosotis sylvatica & 3.907037 & 2620.486 & 33.48145 & 738.3095 & 45.13092 & 55.00734 & 29.83856 & 23.37754 & 20.57718 & 9.192235 & 5.023813 & 16.26698 & 1385.106 & 0.871689 & 267.1451 & 0.742705 \\
\hline Myosurus minimus & 3.294486 & 7524.283 & 135.5345 & 814.6529 & 130.8729 & 111.8895 & 30.05884 & 35.57155 & 53.41206 & 17.65832 & 4.395863 & 10.3264 & 4346.759 & 1.064606 & 438.659 & 0.578238 \\
\hline Myrica gale & 8.807333 & 6673.357 & 139.685 & 445.6634 & 91.82394 & 316.6635 & 67.44289 & 98.19349 & 41.36408 & 31.98927 & 9.181594 & 19.91676 & 5102.597 & 2.337512 & 859.7954 & 1.044292 \\
\hline Nardus stricta & 9.655308 & 4510.646 & 21.07087 & 959.7318 & 90.63048 & 162.6238 & 97.80914 & 129.626 & 37.0178 & 34.89078 & 8.52647 & 7.776709 & 6274.822 & 1.056563 & 407.03 & 1.153672 \\
\hline Narthecium ossifragum & 8.054783 & 3525.793 & 20.36792 & 684.0224 & 57.26137 & 173.5176 & 71.57388 & 107.5417 & 35.53843 & 23.62326 & 9.705506 & 6.292504 & 4056.81 & 2.742625 & 338.6447 & 0.661217 \\
\hline Nuphar lutea & 3.461341 & 5247.198 & 154.2484 & 289.4774 & 82.81307 & 172.3937 & 23.22756 & 34.57458 & 23.33719 & 18.95834 & 10.55466 & 23.28256 & 5104.076 & 1.364818 & 535.5809 & 0.904745 \\
\hline Nymphaea alba & 4.781351 & 5664.907 & 179.2815 & 375.3959 & 103.887 & 144.3111 & 27.85445 & 41.34956 & 28.65503 & 20.25846 & 9.110887 & 40.85259 & 5312.509 & 1.395171 & 553.5522 & 1.132679 \\
\hline Odontites vernus & 3.368011 & 7841.209 & 599.3643 & 517.7048 & 146.8541 & 233.5758 & 55.22235 & 53.76132 & 46.76416 & 26.39963 & 6.401642 & 13.87735 & 3460.373 & 0.834588 & 345.7166 & 0.851534 \\
\hline Odontites vernus s. serotinus & 3.276518 & 7635.679 & 616.7406 & 500.3933 & 119.5469 & 278.7429 & 36.96498 & 41.01268 & 42.13071 & 21.19224 & 6.574519 & 13.71675 & 4652.053 & 1.067789 & 459.5635 & 1.027906 \\
\hline Oenanthe aquatica & 5.634874 & 7074.441 & 207.3381 & 404.5639 & 112.5424 & 211.0295 & .82826 & 54.33912 & 41.01714 & 23.98089 & 1.14981 & 25.89051 & 54.589 & 1.574102 & 79.2662 & 20 \\
\hline
\end{tabular}


Oenanthe fistulosa

Oenanthe lachenal

Oenothera erythrosepala

enothera parvifl

Ononis repens

Ononis repens s. repen

Ononis repens s. spinosa

Orchis militaris

Orchis morio

Origanum vulgare

Ornithogalum umbellatum

Ornithopus perpusillus

Orobanche caryophyllacea

Orobanche picridis

Osmunda regalis

Oxalis acetosel

Oxycoccus palustris

apaver argemone

Papaver rhoeas

Parapholis strigosa

Parentucellia viscos

Paris quadrifolia

Parnassia palustris

Pastinaca sativa

Pedicularis palustris

Pedicularis sylvatic

Persicaria amphibia

Persicaria bisto

Persicaria hydropiper

icaria lapathifolia

Persicaria lapathifolia s. lapathifolia

Persicaria lapathifolia s. pallid

Persicaria maculosa

ersicaria mino

Persicaria mitis

Petasites hybridus

Peucedanum carvifolia

Peucedanum palustre

Phalaris arundinacea

um arenarium

pleum pratense

Phleum pratense s. pratens

Phleum pratense s. serotinum

\begin{tabular}{|c|c|c|c|c|c|c|c|c|c|c|c|c|c|c|c|}
\hline 996 & 2.111 & 272.4001 & 340.8739 & 74.12076 & 202.5195 & 0.98 & 39.50051 & 35.5601 & 20.59331 & 10.11803 & 23.65 & 5700.791 & 1.01217 & 533.4625 & .740 \\
\hline 3.697328 & 6063.47 & 606.7125 & 405.3369 & 122.5005 & 124.2625 & 31.73267 & 31.25643 & 36.30099 & 12.18092 & 5.038128 & 19.49912 & 4441.297 & 1.044246 & 358.5312 & \\
\hline .474024 & 389.734 & 32.5367 & 64.1722 & 121.2473 & 143.5933 & 64.58129 & 63.44364 & 43.2793 & 17.509 & 5.455614 & 25.39144 & 2739.011 & 1.422793 & 435.3505 & \\
\hline 2.42426 & 49.627 & 90.7016 & 82.9172 & 39.0651 & 134.7897 & 43.9622 & 55.68345 & 45.25877 & 17.79234 & 2.158113 & 25.72039 & 2015.142 & 2.227839 & 357.2767 & \\
\hline 899178 & 273.03 & 769.2096 & 89.8678 & 149.663 & 234.0957 & 84.5914 & 106.9586 & 58.78482 & 13.96899 & 2.082888 & 24.81652 & 1897.588 & 1.693998 & 361.3612 & \\
\hline . 278516 & 6168.561 & 47.86131 & 183.644 & 16.7448 & 166.5186 & 88.41909 & 100.6681 & 42.42047 & 23.91619 & 4.643514 & 14.82695 & 2633.064 & 0.729668 & 391.5248 & \\
\hline 957986 & 72.222 & 17.80607 & 216.509 & 7.40745 & 117.3272 & 60.82717 & 53.15079 & 28.60981 & 14.02484 & 5.526835 & 11.52499 & 1877.305 & 1.074446 & 283.5794 & \\
\hline 1.904893 & 51.548 & 01.6842 & 9.5127 & 108.745 & 122.5426 & 45.64644 & 45.84035 & 27.25696 & 12.98195 & 2.615823 & 11.61096 & 2661.063 & 0.849844 & 330.6224 & \\
\hline .776046 & 637.044 & 521.3415 & 405.4721 & 88.75041 & 168.222 & 49.37173 & 46.62102 & 40.59777 & 21.34163 & 5.192136 & 10.71653 & 4983.628 & 1.040659 & 330.5691 & \\
\hline 421359 & 3819.438 & 43.11038 & 694.3385 & 65.94819 & 72.05631 & 41.36147 & 34.38192 & 41.6389 & 8.232593 & 4.977475 & 30.56558 & 1955.751 & 0.486205 & 844.4032 & \\
\hline 711001 & 7861.865 & 103.5794 & 8.5732 & .73171 & 161.9375 & 24471 & 106.1801 & 34.66173 & 37.86072 & 7.047008 & 8.995295 & 6233.501 & 0.65209 & 76.2078 & \\
\hline 3.309733 & 3500.44 & 50.81367 & 0.3936 & 71.86758 & 81.68717 & 44.2379 & 36.39673 & 37.08197 & 8.584432 & 4.003556 & 19.00993 & 2134.403 & 0.561201 & 85.9071 & \\
\hline 367178 & 3767.485 & 46.08565 & 1104.829 & 71.64591 & 74.53801 & 39.36737 & 41.99309 & 19.39278 & 11.6216 & 7.162136 & 26.96569 & 2255.956 & 1.656368 & 332.7979 & \\
\hline 20766 & 3308.865 & 4.53712 & 44.8783 & 35.68387 & 110.6579 & 38.67326 & 52.4085 & 27.07669 & 14.78158 & 3.487801 & 10.15558 & 347.861 & 1.925791 & 30.8427 & \\
\hline 679685 & 3076.941 & 32.62907 & 163.8561 & 87.58108 & 99.34516 & 58.5296 & 47.03318 & 25.23213 & 14.94249 & 3.166535 & 9.402827 & 1968.102 & 1.364286 & 302.7693 & \\
\hline 2.39967 & 1875.722 & 47.00407 & 211.8831 & 40.98084 & 67.32435 & 39.35863 & 39.14702 & 33.30378 & 14.89223 & 5.595398 & 7.662505 & 1760.27 & 1.882348 & 264.5531 & \\
\hline 7.345757 & 5463.151 & 125.6335 & 631.4777 & 85.94017 & 335.4667 & 94.71995 & 150.5139 & 73.5755 & 27.757 & 9.181148 & 28.25005 & 3905.052 & 2.759458 & 759.6512 & \\
\hline 4.64447 & 3372.31 & 39.22079 & 258.0202 & 68.64073 & 101.2912 & 38.42736 & 55.84869 & 27.11164 & 14.45296 & 9.343285 & 22.69182 & 1857.738 & 2.717255 & 170.5615 & \\
\hline 5.423537 & 3724.537 & 26.5311 & 815.7891 & 72.21281 & 296.2158 & 84.4523 & 135.3375 & 35.78131 & 27.48675 & 9.743848 & 6.949633 & 5217.357 & 2.338452 & 516.571 & \\
\hline 247852 & 3238.786 & 69.69486 & 435.3993 & 114.0078 & 87.20505 & 28.39826 & 34.21753 & 39.82266 & 10.08363 & 2.672115 & 9.645838 & 1735.675 & 2.827805 & 291.1578 & \\
\hline .794542 & 3693.857 & 78.52537 & 462.7616 & 107.2246 & 93.29512 & 29.70258 & 35.3778 & 40.18073 & 12.08766 & 2.947483 & 13.13111 & 2010.668 & 3.143776 & 398.4469 & \\
\hline 396675 & 3311.606 & 88.05736 & 601.4325 & 109.6175 & 96.27743 & 27.19323 & 30.62359 & 40.466 & 13.62315 & 3.448966 & 13.90485 & 2411.205 & 2.973405 & 370.8522 & \\
\hline 1.86742 & 29.635 & 536.9816 & 159.6646 & 144.7609 & 246.045 & 39.92663 & 42.67816 & 33.93654 & 20.64772 & 4.807067 & 13.12322 & 2156.021 & 0.561156 & 328.357 & \\
\hline 1.816491 & 57.728 & 596.1088 & 441.6365 & 78.07213 & 133.1502 & 36.38648 & 39.16891 & 27.42961 & 13.26874 & 4.376254 & 11.18802 & 3170.028 & 1.052163 & 252.16 & \\
\hline 1.923671 & 92.785 & 40.24769 & 331.6107 & 45.00416 & 65.23654 & 35.48623 & 26.48027 & 23.48643 & 10.94894 & 3.522604 & 15.24608 & 1614.318 & 0.824972 & 131.8211 & \\
\hline 495584 & 573.356 & 510.917 & 388.5291 & 87.23884 & 201.8114 & 39.48882 & 45.91119 & 32.62341 & 21.21299 & 5.523126 & 11.48294 & 6074.301 & 0.979088 & 339.8553 & \\
\hline 136769 & 4827.147 & 87.75179 & 416.8108 & 104.2091 & 96.04145 & 38.38882 & 43.03432 & 40.82712 & 15.51791 & 3.82023 & 13.30075 & 3341.635 & 1.03635 & 389.1661 & \\
\hline 716456 & 25.296 & 321.7533 & 406.613 & 113.657 & 189.1468 & 31.00583 & 45.65165 & 30.99922 & 22.21573 & 9.044993 & 13.38244 & 4311.102 & 1.541319 & 840.6009 & \\
\hline 782996 & 03.674 & 71048 & 8.4577 & 63.59271 & 72.7516 & 59.29987 & 76.6954 & 31.62734 & 32.12144 & 4.526404 & 7.477512 & 5877.917 & 0.768313 & 280.4894 & \\
\hline .502478 & 238.919 & 230.3805 & 0.3714 & 112.359 & 236.9314 & 54.75676 & 67.44242 & 54.85715 & 31.03756 & 7.820353 & 16.80305 & 8074.078 & 1.035125 & 686.9672 & \\
\hline 5.948022 & 27.399 & .91774 & 6.0358 & 101.4703 & 235.1385 & 58.00612 & 70.81052 & 61.55155 & 1.16479 & 6.479017 & 25.9224 & 4749.832 & 1.434084 & 472.0396 & \\
\hline 881274 & 08.967 & 5.4248 & 497.5667 & 5.5424 & 301.329 & 49.89059 & 75.06853 & 13.5622 & 37.96657 & 8.847655 & 23.32817 & 7128.058 & 1.601286 & 514.766 & \\
\hline 4.761034 & 79.337 & 2.6196 & 577.43 & 0.4842 & 202.7975 & 51.55033 & 65.25027 & 75.5328 & 32.3439 & 6.846348 & 16.67352 & 7632.7 & .525423 & 424.8397 & \\
\hline 1.607799 & 8830.82 & 2.6288 & 578.406 & 9.7905 & 213.7708 & 41.28432 & 56.85597 & 78.12685 & 36.54375 & 5.010904 & 0.85185 & 8902.447 & 1.789891 & 359.4036 & \\
\hline 116127 & 4933.024 & 3.9057 & 4.8165 & 50.9256 & 135.3573 & 48.16636 & 69.91085 & 62.77542 & 13.59856 & 2.961542 & 17.63183 & 3749.863 & 483703 & 433.2964 & \\
\hline 163796 & 349.814 & 4.5274 & 4.1746 & 5.1071 & 188.5567 & 53.92151 & 75427 & 66.80994 & 32.67724 & 6.79459 & 3.05412 & 6370.794 & 178839 & 368.5131 & \\
\hline 7.315105 & 37.529 & 9.8272 & 9.8145 & .1948 & 316.7309 & .31427 & 86.79686 & 21829 & .88624 & .3616 & 28096 & 21.474 & 196128 & 818.7026 & \\
\hline 53177 & 27.295 & 9.3714 & 1.4619 & 3.2283 & 261.1656 & 10871 & 101.5006 & .2109 & 87557 & 11.0477 & 53697 & 48.276 & 408612 & 33.7044 & \\
\hline 821773 & 5693.749 & 190.0109 & 811.8813 & 3.2905 & 145.53 & 57.70932 & 64.51937 & 52.14319 & .36705 & 8.327467 & .31398 & 3976.136 & 557537 & 39.574 & \\
\hline 422715 & 3882.497 & 58.3192 & 0.5906 & .37443 & 65.98186 & 27.94343 & 29.16575 & 25.27079 & .10073 & 1.660327 & 06522 & 45.112 & 671389 & 267.8172 & \\
\hline 501277 & 7231.912 & 4.4482 & 1271 & 105.1748 & 208.6864 & 46.1817 & 88766 & 31.06868 & .94142 & 8.792441 & 29051 & 3556.581 & 682298 & 718.9832 & \\
\hline .23037 & 8736.611 & 3.9638 & .6349 & 3.1419 & 213.783 & 57.76587 & 81.92184 & 48.67965 & . 15776 & 8.197203 & 46989 & 7712.673 & 366598 & 612.5144 & \\
\hline 424363 & 2052.244 & 52812 & 125.8013 & 8442 & 86.16562 & 55.40671 & 50.27771 & 26.25037 & 10.59194 & 3.348277 & 17284 & 1281.987 & 598941 & 214.3336 & \\
\hline 087164 & 7085.975 & .7657 & .7391 & .2797 & 126.075 & 37.02508 & 45.70293 & 36.03696 & 19.24095 & 5.069296 & .56118 & 4380.096 & .273789 & 453.4045 & \\
\hline 9919 & 554.725 & 81494 & 6.0482 & 2.6872 & 91.9043 & 20617 & 38.80521 & 27.24631 & 16.703 & 17579 & 10037 & 3738.823 & 0.735979 & 442.6726 & \\
\hline 2961 & 841.603 & 8.7233 & 1039 & .5455 & 07.8592 & 6.70212 & 75306 & 31.76248 & .88088 & 3.552595 & 0.06575 & 077.593 & 1.30423 & 344.11 & \\
\hline
\end{tabular}


Phragmites australis

Phyteuma spicatum s. nigrum

Picris hieracioides

Pimpinella major

Pimpinella saxifrag

Pinguicula vulgaris

Plantago coronopus

Plantago lanceolat

antago major

Plantago major s. intermedia

Plantago major s. majo

Plantago maritima

Pantago media

Platanthera bifolia

Poa angustifoli

Poa annua

Poa compressa

Poa nemoralis

Poa palustris

pratensis

Poa pratensis + P. angustifolia

Poa trivialis

Polygala comosa

Polygala serpyllifolia

Polygala vulgaris

Polygonatum multiflorum

Polygonatum odoratum

Polygonum aviculare

Polypodium vulgare

Populus alba

Populus tremula

Populus $x$ canescens

Potamogeton crispus

Potamogeton lucen

Potamogeton natans

potamogeton pectinatus

Potamogeton polygonifolius

Potamogeton pusillus

Potentilla anglica

Potentilla anserina

Potentilla argentea

Potentilla erecta

Potentilla palustris

Potentilla reptans

Potentilla sterilis
6.347262

3.323082

3.179093

4.606542

.578376

4.4742 .57

4.158424

3.364899

4.613517

2.478653

4.9395

7.092602

2.399139

4.597304

4.975689

5.781803

7.994095

7.78315

5.909415

7.003252

3.779223

8.907718

6.982764

5.778939

6.505672

7.239335

2.7715

4.901568

3.167941

6.81356
2.338754

6.469801

4.101779

5.074832

7.240479

4.886773

4.473518

5.148283 $\begin{array}{ll}8352.489 & 612.0805\end{array}$

$\begin{array}{lll}8874.492 & 38.52503 & 550.3512\end{array}$

\begin{tabular}{ll}
$532.947 \quad 41.76678$ \\
\hline
\end{tabular}

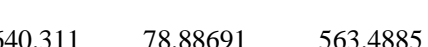

$\begin{array}{lll}6777.744 & 622.334 & 234.3128\end{array}$

$\begin{array}{lll}7768.271 & 124.2141 & 385.7367 \\ 7271.005 & 689.1098 & 536.1895\end{array}$

\begin{tabular}{l}
$723369-6175016-515.5819$ \\
\hline
\end{tabular}

$6920.662 \quad 597.7824 \quad 540.0782$

\begin{tabular}{l}
$697.121 \quad 542.8139$ \\
\hline
\end{tabular}

$\begin{array}{ll}51.70095 & 347.1001\end{array}$

$\begin{array}{lll}714.005 & 162.5642 & 572.3625 \\ \end{array}$

$5079.718 \quad 76.5379$

$7429.924 \quad 515.6447$

$5022.439 \quad 162.1094$

$\begin{array}{ll}2985.185 & 36.86701\end{array}$

244.5372
670.8698

601.4638
408.9369

545.4472

$\begin{array}{lll}8190.457 & 412.7064 & 733.6417\end{array}$

$\begin{array}{lll}2814.597 & 36.93018 & 552.8898\end{array}$

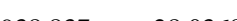

$3927.743 \quad 287.9159$

$\begin{array}{ll}2553.461 & 31.08538\end{array}$

$2232.503 \quad 39.65692$

$2318.653 \quad 40.05092$

$3645.014 \quad 187.617$

$4996.559 \quad 107.2133$

$\begin{array}{ll}3343.222 & 58.8337\end{array}$

$\begin{array}{ll}4337.985 & 69.17009\end{array}$

$4174.775 \quad 172.9359$

$\begin{array}{lll}4512.829 & 213.5884 & 255.9912\end{array}$

$\begin{array}{lll}6305.209 & 213.3497 & 361.2219\end{array}$

$\begin{array}{ll}4754.484 & 252.4582\end{array}$

$\begin{array}{ll}8108.982 & 274.005 \\ 4231.588 & 196.31\end{array}$

$8207.903 \quad 238.4882$

$\begin{array}{ll}6396.697 & 667.8276\end{array}$

$\begin{array}{ll}3010.505 & 26.06378\end{array}$

$\begin{array}{rr}6251.448 & 84.7918 \\ 7126269 & 159.745\end{array}$

$7809.709 \quad 190.1909$

$2824.881 \quad 36.5722$ $\begin{array}{llllllllllll}140.4918 & 260.2474 & 61.31773 & 83.10764 & 50.1183 & 30.03864 & 12.26503 & 33.89231 & 7438.242 & 1.88123 & 857.8412 & 2.152015\end{array}$

$\begin{array}{llllllllllll}13078 & 139.1461 & 73.50981 & 73.14324 & 47.9126 & 29.74739 & 5.90885 & 25.96526 & 4225.741 & 1.891418 & 182.371 & 0.964588\end{array}$

$\begin{array}{llllllllllll}110.2893 & 130.9675 & 57.18461 & 53.63562 & 27.73022 & 17.38282 & 5.815105 & 13.86423 & 2570.425 & 1.151713 & 394.6402 & 0.657543\end{array}$

$\begin{array}{lllllllllllll} & 72.09556 & 39.62711 & 42.73405 & 44.99663 & 14.63646 & 4.025339 & 15.7268 & 2743.842 & 0.590865 & 220.7172 & 0.813198\end{array}$

$\begin{array}{llllllllllll}6.28633 & 277.1957 & 40.50363 & 45.40417 & 23.99385 & 20.20836 & 4.520239 & 9.417776 & 4099.545 & 0.576245 & 257.7584 & 0.927768\end{array}$

$\begin{array}{llllllllllll}197.1845 & 299.1323 & 50.95764 & 55.22858 & 40.2259 & 24.8426 & 5.188294 & 19.45238 & 2820.523 & 1.356926 & 400.2635 & 0.805364\end{array}$

$\begin{array}{lllllllllllll}122.6739 & 166.7908 & 56.2773 & 66.77707 & 34.50731 & 31.22709 & 6.203635 & 13.07495 & 6114.323 & 1.148738 & 484.9156 & 1.211994 \\ 141.8872 & 179.3782 & 39.76927 & 47.93841 & 49.83256 & 21.09777 & 6.176149 & 16.05282 & 4599.914 & 1.694901 & 430.5987 & 1.328069\end{array}$

$\begin{array}{llllllllllll}140.3518 & 175.5188 & 42.82602 & 50.21431 & 46.81139 & 29.67384 & 6.349774 & 16.82544 & 6711.444 & 1.183891 & 558.1731 & 1.111217\end{array}$

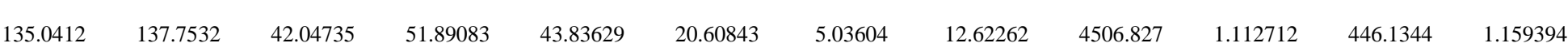

$\begin{array}{llllllllllll}128.0874 & 203.5779 & 35.4864 & 34.93976 & 32.425 & 15.43949 & 5.359103 & 11.17076 & 2808.452 & 0.71074 & 414.1811 & 0.854728\end{array}$

$\begin{array}{llllllllllll}89.2053 & 92.99998 & 43.108 & 42.65183 & 21.36522 & 12.47068 & 3.1066 & 9.175729 & 2722.253 & 0.662531 & 306.2595 & 0.494922\end{array}$

$\begin{array}{llllllllllll}77.00686 & 182.4887 & 52.56058 & 71.80818 & 23.97006 & 32.56256 & 5.304953 & 9.931336 & 5595.824 & 1.023778 & 349.7526 & 0.854338\end{array}$

$\begin{array}{llllllllllll}124.5886 & 132.9906 & 44.08775 & 52.41792 & 41.04329 & 17.48955 & 5.432434 & 16.39329 & 3308.425 & 0.746809 & 400.2185 & 1.062683\end{array}$

$\begin{array}{llllllllllll}183.8809 & 153.6167 & 47.3437 & 60.62467 & 46.32957 & 20.297 & 5.774088 & 15.04167 & 4810.65 & 1.758199 & 597.8899 & 1.776923\end{array}$

$\begin{array}{llllllllllll}110.0315 & 186.6335 & 96.57969 & 84.31965 & 77.6273 & 17.96244 & 6.434719 & 27.99503 & 2860.81 & 0.477426 & 400.5695 & 1.15302\end{array}$

$\begin{array}{llllllllllll}60.58622 & 89.82511 & 35.33812 & 39.66927 & 30.17881 & 10.7646 & 5.937992 & 18.94405 & 1942.633 & 1.439212 & 152.6157 & 1.08072\end{array}$

$\begin{array}{llllllllllll}127.0049 & 230.5646 & 56.81468 & 71.8622 & 51.84716 & 28.27716 & 10.02518 & 29.39323 & 7011.207 & 1.585037 & 719.2426 & 1.174065\end{array}$

$\begin{array}{llllllllllll}135.4414 & 47.26968 & 63.32637 & 45.45314 & 20.17498 & 7.315317 & 17.1559 & 4488.292 & 2.068325 & 426.8775 & 1.822869\end{array}$

$\begin{array}{llllllllllll}137.4693 & 165.5375 & 50.58338 & 64.53929 & 57.3665 & 26.9626 & 8.31867 & 24.02051 & 5064.763 & 1.484894 & 409.9265 & 1.442905\end{array}$

$\begin{array}{llllllllllll}60.10919 & 72.62224 & 27.55846 & 28.13327 & 20.64946 & 6.299628 & 1.950486 & 12.31293 & 2157.081 & 0.476408 & 288.0453 & 0.533125\end{array}$

$\begin{array}{llllllllllll}72.66282 & 198.0985 & 69.78893 & 103.5117 & 37.42595 & 33.04016 & 5.350751 & 7.87677 & 6139.062 & 0.821772 & 261.3805 & 0.893314\end{array}$

$\begin{array}{lllllllllllll}83.03628 & 135.2507 & 74.50254 & 76.28256 & 47.14218 & 21.45061 & 5.364071 & 10.6617 & 2428.94 & 0.891071 & 270.1355 & 1.65397\end{array}$

$\begin{array}{llllllllllll}54.08171 & 88.21047 & 36.45821 & 47.10701 & 29.45301 & 10.75472 & 8.739981 & 25.65766 & 1605.575 & 2.440213 & 172.9964 & 1.239556\end{array}$

$\begin{array}{llllllllllll}74.68034 & 81.9712 & 33.90407 & 38.63304 & 28.18955 & 11.38218 & 6.913296 & 24.95229 & 1922.993 & 1.301493 & 323.006 & 1.507969\end{array}$

$\begin{array}{llllllllllll}144.9066 & 136.5558 & 34.57654 & 44.14262 & 48.1124 & 18.77486 & 4.704999 & 13.62156 & 4445.902 & 2.62276 & 423.3283 & 1.365881\end{array}$

$\begin{array}{llllllllllll}63.02342 & 89.58916 & 48.72076 & 70.12196 & 34.61352 & 12.43002 & 11.89466 & 19.41753 & 2129.206 & 3.666706 & 374.0701 & 2.098543\end{array}$

$\begin{array}{llllllllllll}97.8066 & 138.9576 & 48.43236 & 57.9918 & 30.84858 & 17.938 & 7.972503 & 31.90316 & 3623.128 & 1.654295 & 449.718 & 2.202651\end{array}$

$\begin{array}{llllllllllll}120.1035 & 164.3373 & 65.08304 & 71.49332 & 46.84913 & 25.38027 & 7.246564 & 23.08998 & 4418.674 & 1.303545 & 588.1237 & 1.938796\end{array}$

$\begin{array}{llllllllllll}72.67822 & 110.232 & 50.35387 & 68.13429 & 49.97907 & 16.75753 & 9.466916 & 27.0862 & 3643.765 & 2.48076 & 454.374 & 1.34165\end{array}$

\begin{tabular}{|l|l|l|l|l|l|l|l|l|l|} 
&
\end{tabular}

\begin{tabular}{llllllllllll}
66.3515 & 197.3814 & 20.51406 & 25.7828 & 29.95348 & 16.14685 & 5.685788 & 20.06927 & 4577.791 & 1.144331 & 446.216 & 0.525095 \\
\hline
\end{tabular}

$\begin{array}{llllllllllll}67.78253 & 184.428 & 22.88367 & 33.1259 & 25.158 & 14.92713 & 9.207709 & 21.45128 & 4505.414 & 1.022296 & 471.4289 & 0.715927\end{array}$

$\begin{array}{llllllllllll}118.5866 & 180.3559 & 29.7658 & 39.81326 & 32.83368 & 19.06801 & 11.54902 & 22.91365 & 5057.992 & 1.527475 & 510.0661 & 1.08579\end{array}$

$\begin{array}{llllllllllll}81.96426 & 170.6092 & 21.13186 & 25.98612 & 34.29516 & 18.98026 & 5.460908 & 15.4044 & 4732.826 & 1.023644 & 450.9135 & 0.751688\end{array}$

$\begin{array}{llllllllllll}127.8726 & 231.7171 & 15.21282 & 28.7584 & 17.00497 & 25.94416 & 9.606583 & 17.90604 & 6996.28 & 0.609022 & 938.5649 & 1.118069\end{array}$

$\begin{array}{llllllllllll}71.84342 & 173.7993 & 16.46971 & 19.18023 & 27.86165 & 16.8003 & 4.900763 & 11.68985 & 4545.93 & 0.931397 & 486.5986 & 0.601481\end{array}$

$\begin{array}{llllllllllll}99.94069 & 223.9869 & 60.5054 & 79.55111 & 44.99106 & 23.44864 & 7.870486 & 12.31748 & 5314.838 & 1.628719 & 440.1394 & 0.977731\end{array}$

$\begin{array}{llllllllllll}113.2148 & 155.682 & 47.1127 & 42.82666 & 51.95101 & 19.88364 & 5.794162 & 14.47964 & 5285.016 & 1.048923 & 508.0021 & 1.263122\end{array}$

$\begin{array}{llllllllllll}79.69735 & 97.48652 & 41.33109 & 54.14327 & 27.78136 & 10.81987 & 3.161315 & 9.258436 & 1830.872 & 1.679094 & 269.5199 & 1.259874\end{array}$

$\begin{array}{llllllllllll}92.25729 & 233.718 & 71.15508 & 98.53853 & 35.4036 & 29.916 & 5.961056 & 11.02935 & 5777.981 & 1.474953 & 333.4225 & 1.164095\end{array}$

$\begin{array}{llllllllllll}121.512 & 180.0475 & 32.28027 & 49.93811 & 29.93814 & 22.85221 & 10.00362 & 28.55837 & 3648.264 & 1.928175 & 715.4344 & 0.774625\end{array}$

$\begin{array}{llllllllllll}162.1692 & 146.7399 & 57.48319 & 65.14842 & 49.95216 & 26.61017 & 4.820917 & 15.41291 & 5533.448 & 0.886798 & 632.7605 & 0.605627\end{array}$

$\begin{array}{llllllllllll}50.18585 & 55.34969 & 40.9601 & 33.1423 & 23.20722 & 10.28751 & 5.944406 & 14.24549 & 1496.794 & 1.448087 & 196.8251 & 1.157818\end{array}$ 


\begin{tabular}{|c|c|c|c|c|c|c|c|c|c|c|c|c|c|c|c|c|}
\hline otentilla verna & 1.567092 & 2775.205 & 27.42183 & 165.0552 & 69.09086 & 85.45043 & 42.52986 & 46.09902 & 28.96071 & 12.33701 & 4.30699 & 10.22757 & 1529.962 & 0.66421 & 223.1253 & 0.844688 \\
\hline Primula elatior & 3.818612 & 5407.316 & 44.12162 & 393.9035 & 58.49615 & 122.5356 & 50.55874 & 47.27798 & 43.69253 & 24.68218 & 3.66583 & 16.99257 & 3168.612 & 0.983771 & 136.8604 & 0.71248 \\
\hline Primula veris & 8.853643 & 4280.057 & 57.34984 & 900.5861 & 80.54711 & 81.72424 & 60.9171 & 51.77325 & 44.51038 & 14.54024 & 6.766278 & 30.59782 & 2730.081 & 0.795384 & 350.1284 & 1.58378 \\
\hline Prunella vulgaris & 5.260507 & 7156.868 & 378.974 & 424.6008 & 99.87509 & 180.6012 & 53.79357 & 60.96541 & 35.82852 & 24.9665 & 5.337998 & 13.40449 & 5803.701 & 0.826391 & 409.51 & 1.267795 \\
\hline Prunus avium & 4.646817 & 2606.122 & 38.73992 & 452.56 & 53.00856 & 85.30346 & 38.66795 & 40.35878 & 29.76261 & 11.21015 & 7.772391 & 20.88023 & 1774.561 & 2.775633 & 216.5443 & 1.075055 \\
\hline Prunus padus & 4.741312 & 3399.614 & 45.40222 & 434.358 & 65.66959 & 126.366 & 40.12736 & 51.18927 & 44.81935 & 15.37576 & 7.41741 & 19.74419 & 2738.263 & 1.530462 & 239.6239 & 1.061261 \\
\hline Prunus spinosa & 6.166444 & 3258.748 & 59.59539 & 958.9323 & 69.96952 & 90.78148 & 36.78629 & 39.97354 & 37.04845 & 12.05752 & 7.377938 & 22.64582 & 2648.793 & 2.314939 & 347.7931 & 1.748054 \\
\hline Pteridium aquilinum & 6.254935 & 2045.596 & 35.80602 & 499.3945 & 59.00225 & 87.99969 & 54.10096 & 75.25064 & 32.60352 & 13.65886 & 6.09303 & 24.95444 & 1694.085 & 1.702902 & 436.8008 & 1.133686 \\
\hline Puccinellia distans s. distans & 2.598904 & 5596.847 & 642.0198 & 415.6636 & 86.1677 & 192.6803 & 25.15237 & 28.66881 & 29.39919 & 15.94299 & 5.412598 & 13.09755 & 4392.95 & 1.524591 & 413.5097 & 0.89328 \\
\hline Puccinellia maritima & 1.674002 & 5671.799 & 561.031 & 524.887 & 71.99597 & 183.344 & 24.39914 & 25.53519 & 33.849 & 22.50162 & 5.256253 & 17.38415 & 3103.315 & 1.602469 & 332.2997 & 0.709391 \\
\hline Pulicaria dysenterica & 3.538377 & 7256.442 & 598.9502 & 459.7941 & 113.5607 & 201.3327 & 54.71123 & 46.73398 & 52.45487 & 18.45278 & 5.21876 & 16.67106 & 5264.158 & 1.338419 & 359.0327 & 1.888973 \\
\hline Pyrola minor & 7.290674 & 4397.878 & 346.1274 & 642.8071 & 90.25355 & 249.2346 & 98.35804 & 127.907 & 60.97688 & 16.5584 & 9.974683 & 10.7589 & 3281.554 & 1.686064 & 358.6996 & 1.595046 \\
\hline Pyrola rotundifolia & 4.866422 & 4797.275 & 446.027 & 353.331 & 87.13033 & 216.239 & 74.69027 & 81.91122 & 46.26493 & 21.00233 & 5.87736 & 12.63656 & 3324.406 & 1.457082 & 293.048 & 1.250977 \\
\hline Pyrus communis & 10.0321 & 5317.362 & 60.32238 & 900.6508 & 118.4424 & 123.7593 & 68.18661 & 61.60929 & 38.97183 & 22.53783 & 13.29579 & 31.05863 & 3516.162 & 3.199138 & 445.4979 & 2.247287 \\
\hline Quercus petraea & 7.135671 & 1938.554 & 40.68773 & 519.291 & 64.76407 & 88.90781 & 48.65884 & 70.13922 & 34.81967 & 14.95407 & 9.760814 & 35.90756 & 1696.776 & 4.125824 & 463.0025 & 1.043073 \\
\hline Quercus robur & 8.737833 & 3305.68 & 50.115 & 937.3913 & 79.45509 & 120.9629 & 67.05922 & 91.08571 & 51.46984 & 18.49964 & 11.89638 & 34.69302 & 3596.597 & 2.787006 & 506.0109 & 1.618282 \\
\hline Radiola linoides & 4.963377 & 6249.662 & 650.8414 & 274.8425 & 108.0469 & 282.2338 & 56.11162 & 60.15157 & 42.89531 & 14.08826 & 4.194542 & 9.932909 & 3550.057 & 0.662078 & 255.9909 & 1.309365 \\
\hline Ranunculus acris & 4.677883 & 6378.436 & 101.2198 & 393.5177 & 102.1173 & 126.037 & 38.51346 & 46.70939 & 34.59407 & 22.76605 & 5.012823 & 13.83456 & 4407.164 & 0.838716 & 390.5592 & 0.959029 \\
\hline Ranunculus aquatilis & 3.714846 & 7006.016 & 314.835 & 674.821 & 108.0225 & 208.0204 & 33.78872 & 44.13084 & 37.88099 & 20.66551 & 6.392288 & 22.57565 & 5378.799 & 1.16342 & 534.1725 & 1.140055 \\
\hline Ranunculus arvensis & 3.106157 & 3348.439 & 80.91843 & 815.6457 & 123.08 & 78.06776 & 21.18552 & 25.07842 & 46.95829 & 10.79347 & 3.081587 & 9.896207 & 1780.976 & 1.258431 & 218.9016 & 0.470867 \\
\hline Ranunculus auricomus & 6.399654 & 8719.485 & 61.94191 & 667.1923 & 93.36625 & 108.8064 & 68.53819 & 65.55443 & 47.85109 & 27.5071 & 5.538896 & 26.70241 & 4008.55 & 1.585994 & 186.2535 & 0.848702 \\
\hline Ranunculus bulbosus & 2.365549 & 4845.927 & 82.71438 & 293.965 & 111.7747 & 104.4211 & 48.25817 & 51.84231 & 24.58784 & 15.44404 & 2.715513 & 10.41806 & 2938.14 & 0.990595 & 342.5872 & 0.820708 \\
\hline Ranunculus circinatus & 2.545143 & 5401.906 & 237.5742 & 568.3561 & 85.2319 & 187.5242 & 19.95794 & 24.81883 & 26.08375 & 17.28748 & 6.019024 & 16.28339 & 4603.673 & 0.950023 & 473.5442 & 0.617687 \\
\hline Ranunculus ficaria & 5.705589 & 7132.617 & 106.4626 & 713.7471 & 95.02052 & 126.0332 & 54.97911 & 57.82947 & 27.83245 & 27.52746 & 6.413912 & 19.741 & 4038.007 & 1.56966 & 221.2025 & 1.000727 \\
\hline Ranunculus ficaria s. bulbilifer & 7.398141 & 6597.319 & 98.1584 & 837.1608 & 119.3008 & 130.4436 & 52.13979 & 59.78955 & 43.87528 & 21.24712 & 7.22193 & 21.00171 & 3558.361 & 1.284896 & 242.8452 & 1.222525 \\
\hline Ranunculus flammula & 4.39397 & 6019.272 & 372.9256 & 406.3957 & 105.925 & 159.3888 & 35.25225 & 55.68924 & 37.60892 & 20.30564 & 5.785567 & 15.02769 & 4560.885 & 1.154665 & 808.7943 & 0.821935 \\
\hline Ranunculus lingua & 3.324727 & 4469.146 & 145.358 & 403.3325 & 93.21311 & 137.3816 & 22.06755 & 33.8348 & 20.18591 & 20.69809 & 9.10326 & 28.36125 & 4319.986 & 1.049853 & 514.4752 & 0.692839 \\
\hline Ranunculus peltatus & 3.648846 & 4460.851 & 186.0296 & 382.7917 & 85.28654 & 191.1214 & 33.48331 & 46.48582 & 38.64675 & 20.51074 & 5.044803 & 17.99867 & 5577.629 & 1.095041 & 429.6756 & 0.701054 \\
\hline Ranunculus repens & 6.563128 & 7690.959 & 214.2075 & 482.0557 & 128.8739 & 155.6109 & 46.96343 & 60.00616 & 44.3201 & 26.49834 & 6.369168 & 20.41536 & 5386.596 & 1.279981 & 458.7515 & 1.094702 \\
\hline Ranunculus sardous & 1.849421 & 4979.176 & 569.1036 & 568.0818 & 86.73654 & 152.4326 & 28.91575 & 33.02308 & 36.91749 & 16.40714 & 3.427474 & 10.10009 & 3883.777 & 1.216744 & 574.4655 & 0.809457 \\
\hline Ranunculus sceleratus & 4.503435 & 7341.761 & 458.0769 & 525.2169 & 105.5635 & 222.959 & 40.47521 & 56.32983 & 45.56454 & 30.7092 & 7.849657 & 20.98024 & 7511.29 & 1.22685 & 544.9345 & 1.15611 \\
\hline Raphanus raphanistrum & 3.378259 & 4093.677 & 95.79519 & 681.303 & 101.4899 & 114.9266 & 29.26497 & 38.84478 & 42.27464 & 13.10599 & 3.191033 & 20.82197 & 2863.859 & 2.65766 & 334.8715 & 0.868745 \\
\hline Reseda lutea & 2.456048 & 4150.416 & 86.93664 & 434.2006 & 120.399 & 93.97644 & 41.52833 & 45.65707 & 35.20201 & 16.08974 & 3.207125 & 18.56289 & 2826.371 & 2.514142 & 423.7955 & 0.613967 \\
\hline Reseda luteola & 3.248155 & 3794.718 & 125.1459 & 480.2561 & 154.6368 & 99.00299 & 42.72703 & 40.50789 & 46.5887 & 15.97868 & 3.304019 & 30.54191 & 2558.833 & 2.120947 & 512.6873 & 0.691037 \\
\hline Rhamnus cathartica & 6.132295 & 5354.503 & 442.8266 & 671.5149 & 87.89926 & 245.5493 & 68.61673 & 74.05767 & 67.90577 & 21.0675 & 8.443267 & 29.29412 & 3764.56 & 1.868047 & 384.183 & 1.69046 \\
\hline Rhamnus frangula & 7.131726 & 3323.815 & 59.81068 & 578.5179 & 73.01669 & 146.8413 & 79.514 & 101.8475 & 56.7585 & 20.10193 & 9.274463 & 25.493 & 3716.493 & 2.426851 & 478.7113 & 1.349789 \\
\hline Rhinanthus alectorolophus & 1.574454 & 3274.779 & 45.96719 & 480.4742 & 60.35948 & 57.55878 & 63.0509 & 40.28521 & 36.63582 & 8.324258 & 3.119097 & 7.927986 & 1343.585 & 0.447423 & 207.6887 & 0.611699 \\
\hline Rhinanthus angustifolius & 3.809205 & 8854.355 & 363.2867 & 452.3742 & 143.9331 & 219.4393 & 60.19152 & 61.38925 & 30.07753 & 28.42732 & 7.235419 & 10.5131 & 7585.637 & 0.913195 & 728.6457 & 1.015652 \\
\hline Rhinanthus minor & 3.366494 & 8452.766 & 363.9978 & 375.324 & 125.6256 & 185.084 & 72.5905 & 75.21865 & 48.2957 & 27.73745 & 5.830134 & 11.21272 & 4553.172 & 0.682092 & 403.7874 & 1.102729 \\
\hline Rhynchospora alba & 6.579881 & 3097.723 & 10.23213 & 761.3556 & 61.18857 & 152.0516 & 63.7001 & 74.6441 & 31.68743 & 22.33782 & 10.44233 & 5.383307 & 3662.009 & 2.503708 & 230.8563 & 0.508988 \\
\hline Rhynchospora fusca & 7.484133 & 3415.439 & 12.03476 & 798.0216 & 64.43383 & 127.5429 & 59.1939 & 75.5494 & 30.73753 & 21.72862 & 6.784937 & 5.474439 & 3253.785 & 2.337103 & 214.6039 & 0.598245 \\
\hline Ribes nigrum & 3.865064 & 4070.392 & 63.33313 & 303.3114 & 73.08802 & 156.3365 & 46.9507 & 57.45084 & 44.45409 & 17.12676 & 7.068789 & 17.99532 & 3110.439 & 1.000122 & 251.9827 & 0.804068 \\
\hline Ribes rubrum & 4.292367 & 3182.178 & 50.62972 & 446.2121 & 62.96665 & 117.0642 & 40.98387 & 44.8431 & 37.7873 & 14.78499 & 6.072278 & 16.84215 & 2894.946 & 1.39225 & 256.6269 & 1.007391 \\
\hline Ribes uva-crispa & 4.556567 & 2638.784 & 42.01425 & 617.7042 & 55.58278 & 90.44495 & 37.21882 & 36.70095 & 24.18319 & 11.38535 & 5.00332 & 20.70616 & 2051.813 & 1.707869 & 267.0518 & 1.095439 \\
\hline Rorippa amphibia & 4.57634 & 6051.73 & 179.1788 & 395.7354 & 94.61453 & 218.9322 & 37.64853 & 49.76737 & 40.07259 & 24.07168 & 9.787513 & 25.26909 & 5262.697 & 1.197564 & 541.951 & 0.917779 \\
\hline Rorippa microphylla & 3.689153 & 5823.808 & 186.7586 & 455.8466 & 93.37516 & 201.8041 & 29.03658 & 38.13903 & 35.05152 & 20.9257 & 6.371945 & 24.99137 & 5292.808 & 1.181756 & 514.4058 & 41 \\
\hline
\end{tabular}




\begin{tabular}{|c|c|c|c|c|c|c|c|c|c|c|c|c|c|c|c|c|}
\hline um-aquaticum & 3.098199 & 5093.018 & 514.2884 & 391.6292 & 76.23312 & 207.411 & 29.50477 & 47.51637 & 35.40117 & 22.70514 & 5.583631 & 23.36254 & 6119.073 & 1.098997 & 541.9395 & 2492 \\
\hline Rorippa nasturtium-aquaticum & 3.3362 & 6177.235 & 401.5606 & 406.746 & 101.9527 & 215.4133 & 46.71071 & 62.38219 & 48.91898 & 25.78136 & 6.773203 & 24.26006 & 6600.795 & 1.088886 & 619.2702 & 0.99177 \\
\hline Rorippa palustris & 5.005445 & 7628.704 & 372.0893 & 490.524 & 122.2345 & 191.1526 & 54.36985 & 72.07734 & 59.37047 & 29.90219 & 8.50165 & 22.92401 & 7138.867 & 1.477327 & 418.0779 & 1.309596 \\
\hline Rorippa sylvestris & 3.469467 & 7073.602 & 174.1501 & 460.9708 & 86.87432 & 116.0567 & 40.14224 & 50.22291 & 45.23165 & 28.03148 & 5.689396 & 11.51078 & 7237.8 & 1.039383 & 345.9321 & 0.69346 \\
\hline Rosa canina & 6.327583 & 3652.007 & 68.00413 & 773.0294 & 81.22301 & 102.0183 & 38.45037 & 43.68749 & 40.19669 & 14.8181 & 6.747757 & 26.40218 & 3266.365 & 1.933702 & 405.366 & 1.778872 \\
\hline Rosa pimpinellifolia & 5.430819 & 2274.409 & 48.30207 & 282.2586 & 53.68065 & 97.24682 & 54.29842 & 54.93368 & 37.85717 & 12.5778 & 6.765715 & 16.87782 & 1827.386 & 1.6076 & 310.7714 & 1.632969 \\
\hline Rosa rubiginosa & 5.254809 & 3022.409 & 112.6989 & 574.1052 & 70.85006 & 99.21602 & 35.29862 & 42.30314 & 33.91385 & 12.78809 & 5.716799 & 25.63568 & 2208.248 & 1.608477 & 298.734 & 1.269443 \\
\hline Rosa rugosa & 6.712145 & 2803.229 & 585.2516 & 668.2534 & 78.71305 & 134.0513 & 42.07354 & 51.31671 & 45.23217 & 11.72709 & 8.435806 & 23.55438 & 1803.116 & 2.192122 & 270.9671 & 1.956167 \\
\hline Rosa villosa & 4.67768 & 2726.024 & 99.95421 & 559.372 & 59.7221 & 91.43145 & 34.31951 & 39.69208 & 47.54689 & 17.75894 & 9.333311 & 35.81355 & 4157.124 & 2.271334 & 324.8326 & 1.564953 \\
\hline Rubus caesius & 7.941604 & 4378.908 & 234.4657 & 785.0789 & 118.6903 & 168.9606 & 65.06138 & 67.13988 & 55.28125 & 22.89389 & 8.005363 & 31.6519 & 3938.298 & 1.931439 & 462.2851 & 1.671461 \\
\hline Rubus corylifolius & 11.67497 & 5693.829 & 86.27572 & 947.1856 & 153.1775 & 156.6302 & 84.96208 & 111.0732 & 43.43458 & 21.45483 & 12.65219 & 36.94147 & 2625.333 & 2.86401 & 451.7317 & 2.496048 \\
\hline Rubus fruticosus ag. & 7.67161 & 3731.211 & 70.10259 & 797.4378 & 80.89803 & 146.8959 & 61.8526 & 84.80787 & 49.66738 & 20.05304 & 11.17968 & 32.13625 & 3548.124 & 2.62015 & 418.2832 & 1.574997 \\
\hline Rubus gratus & 6.962221 & 2057.988 & 44.02035 & 471.2326 & 59.64631 & 82.48256 & 51.26829 & 64.87797 & 33.54885 & 12.27823 & 10.24806 & 22.28218 & 2157.824 & 1.721486 & 553.4623 & 1.153744 \\
\hline Rubus idaeus & 5.872365 & 3679.655 & 54.28298 & 369.7214 & 74.82369 & 142.327 & 49.32488 & 69.25403 & 43.12198 & 18.3941 & 8.422315 & 27.65689 & 2886.209 & 2.20651 & 249.4106 & 1.329809 \\
\hline Rubus plicatus & 8.646212 & 1814.388 & 43.04812 & 1089.641 & 59.89568 & 83.05714 & 55.81649 & 70.83238 & 43.18515 & 12.46012 & 13.39052 & 13.08018 & 2797.232 & 2.948773 & 661.5643 & 1.352274 \\
\hline Rubus pyramidalis & 4.393184 & 1357.844 & 25.85694 & 408.6821 & 35.20402 & 64.87556 & 32.37574 & 43.83608 & 31.73434 & 9.228643 & 9.403307 & 16.8598 & 1633.944 & 1.620349 & 544.7774 & 0.723826 \\
\hline Rubus ulmifolius & 5.625807 & 5710.642 & 174.7982 & 787.6532 & 177.7925 & 150.5742 & 42.94052 & 56.92743 & 34.71956 & 16.61123 & 5.053474 & 23.22074 & 3848.876 & 2.757017 & 342.8521 & 2.116069 \\
\hline Rumex acetosa & 6.036778 & 8136.581 & 96.32035 & 437.4596 & 122.5428 & 169.3195 & 46.10718 & 58.89895 & 34.9194 & 27.08725 & 6.043134 & 14.22605 & 5677.541 & 1.252677 & 517.4201 & 1.207582 \\
\hline Rumex acetosella & 10.20791 & 3491.833 & 42.64592 & 541.7114 & 88.41874 & 107.7862 & 70.13978 & 90.18461 & 35.99952 & 16.66975 & 7.013294 & 12.84301 & 2940.04 & 3.004855 & 465.0986 & 1.76183 \\
\hline Rumex conglomeratus & 5.803296 & 8258.885 & 332.6386 & 668.0573 & 131.2237 & 159.1402 & 59.86162 & 72.77884 & 71.27312 & 27.39494 & 6.100013 & 20.44906 & 6438.22 & 1.00178 & 529.6392 & 1.174341 \\
\hline Rumex cripsus x obtusifolius & 2.767463 & 5487.632 & 166.2307 & 438.7657 & 103.2092 & 112.8413 & 33.99194 & 39.56985 & 38.55048 & 19.41862 & 4.237952 & 9.859209 & 4653.669 & 0.70459 & 374.501 & 0.605947 \\
\hline Rumex crispus & 3.624253 & 8171.961 & 419.7925 & 513.211 & 127.5608 & 135.6747 & 44.98304 & 51.86277 & 45.25484 & 26.77107 & 5.479406 & 13.1496 & 6946.163 & 0.983474 & 578.4639 & 0.988859 \\
\hline Rumex hydrolapathum & 3.948387 & 6041.177 & 201.018 & 427.0381 & 107.3528 & 189.4512 & 36.63523 & 50.1345 & 34.53793 & 23.34756 & 10.0468 & 25.28529 & 6037.402 & 1.048539 & 614.8364 & 1.016653 \\
\hline Rumex maritimus & 3.093661 & 8142.507 & 471.8407 & 465.8869 & 113.0584 & 152.1163 & 45.00509 & 60.80082 & 48.28426 & 32.41784 & 5.673088 & 18.43874 & 9324.462 & 1.328719 & 523.3269 & 0.890445 \\
\hline Rumex obtusifolius & 6.856309 & 6481.817 & 123.5714 & 660.4036 & 141.1069 & 125.0641 & 42.64705 & 54.82818 & 51.16678 & 20.08444 & 6.987503 & 21.95529 & 4143.384 & 1.365439 & 312.8548 & 1.271055 \\
\hline Rumex obtusifolius s. obtusifolius & 7.077475 & 7171.032 & 175.2766 & 683.9842 & 132.5163 & 144.6162 & 53.25719 & 69.89416 & 61.06027 & 29.16593 & 7.934391 & 24.19557 & 5654.835 & 1.176294 & 335.7836 & 1.194518 \\
\hline Rumex palustris & 4.982061 & 7433.909 & 504.9638 & 505.6838 & 96.63752 & 182.1001 & 46.00583 & 62.91819 & 51.46053 & 32.9579 & 8.22559 & 18.81878 & 8262.171 & 1.74901 & 474.1592 & 1.181198 \\
\hline Rumex sanguineus & 4.551764 & 4460.347 & 115.5639 & 559.7956 & 76.44 & 121.7769 & 46.86044 & 51.19505 & 43.07165 & 20.62816 & 4.985649 & 16.20457 & 3581.442 & 0.903738 & 243.2523 & 0.979436 \\
\hline Rumex thyrsiflorus & 1.410561 & 3811.992 & 62.61739 & 331.1474 & 81.77864 & 74.30983 & 33.89857 & 37.10725 & 30.13886 & 12.40901 & 2.474039 & 8.992942 & 2461.936 & 1.035066 & 257.9986 & 0.467539 \\
\hline Sagina apetala & 3.260891 & 6971.68 & 690.5674 & 338.7626 & 173.9628 & 216.2471 & 77.35127 & 84.18028 & 62.13205 & 17.65855 & 3.54277 & 14.81638 & 2892.771 & 2.690856 & 455.0585 & 1.2915 \\
\hline Sagina maritima & 1.610218 & 5808.939 & 608.7662 & 175.1195 & 176.8483 & 329.9158 & 46.15816 & 52.32101 & 30.28915 & 35.92136 & 4.547599 & 17.1964 & 2425.713 & 0.713859 & 360.9823 & 0.688922 \\
\hline Sagina nodosa & 4.019053 & 9967.447 & 596.6867 & 302.6531 & 163.372 & 331.5703 & 76.28395 & 79.62673 & 52.81163 & 32.62544 & 7.675878 & 13.81768 & 5596.379 & 0.864624 & 447.7376 & 1.21876 \\
\hline Sagina procumbens & 4.114885 & 9464.01 & 578.5852 & 450.1838 & 186.1715 & 237.1128 & 54.62024 & 63.19172 & 49.51423 & 28.47523 & 6.989932 & 15.68226 & 5818.646 & 1.498165 & 498.2362 & 1.39043 \\
\hline Sagittaria sagitttifolia & 2.582576 & 4481.243 & 195.4868 & 298.8384 & 69.87721 & 182.8193 & 17.78138 & 21.50058 & 22.42842 & 16.00571 & 11.3684 & 23.88791 & 4531.706 & 0.934619 & 490.0854 & 0.616217 \\
\hline Salix alba & 5.198666 & 6051.429 & 228.6305 & 771.9721 & 124.5886 & 145.5068 & 60.19177 & 69.32356 & 59.16912 & 26.03473 & 6.631602 & 19.59756 & 5269.148 & 1.800797 & 372.9033 & 1.605593 \\
\hline Salix aurita & 6.469929 & 5618.299 & 191.8775 & 588.435 & 87.02325 & 289.4413 & 77.8527 & 115.2231 & 58.21194 & 28.29444 & 9.481431 & 30.11491 & 4767.209 & 2.37057 & 609.5943 & 1.062941 \\
\hline Salix aurita + S. cinerea & 3.399032 & 3290.775 & 109.4994 & 212.6037 & 66.91287 & 263.5864 & 54.36288 & 63.84594 & 24.50315 & 16.55422 & 4.780017 & 16.6857 & 2878.814 & 0.892778 & 352.2215 & 0.693956 \\
\hline Salix aurita x cinerea & 5.308014 & 7979.2 & 174.5765 & 482.9738 & 119.1058 & 229.7504 & 64.51162 & 91.00621 & 37.1745 & 22.74731 & 7.883091 & 28.91153 & 4108.291 & 2.441596 & 736.5403 & 0.944764 \\
\hline Salix caprea & 7.181795 & 5153.666 & 400.3934 & 834.1309 & 100.0117 & 225.9756 & 73.66886 & 87.39089 & 65.41807 & 21.63495 & 10.03174 & 33.5801 & 3712.949 & 2.87627 & 365.8531 & 2.153354 \\
\hline Salix cinerea & 5.835026 & 6209.761 & 307.0701 & 620.4355 & 98.55733 & 285.313 & 73.50701 & 100.7294 & 55.66443 & 23.34337 & 10.8555 & 32.85579 & 4588.837 & 1.935497 & 648.2195 & 1.262264 \\
\hline Salix fragilis & 4.91742 & 6216.671 & 193.3485 & 573.4351 & 119.8126 & 157.2874 & 68.48809 & 75.56664 & 71.93337 & 25.78902 & 7.156194 & 19.85178 & 6339.924 & 1.94924 & 404.909 & 1.468191 \\
\hline Salix pentandra & 5.747529 & 9359.755 & 213.4004 & 862.4328 & 158.4178 & 271.6649 & 67.07533 & 97.23711 & 57.56606 & 33.00665 & 11.93654 & 32.12427 & 8140.219 & 2.156234 & 868.3432 & 1.833165 \\
\hline Salix purpurea & 4.615952 & 9392.805 & 554.1961 & 755.4252 & 148.4054 & 242.1327 & 81.18269 & 98.35505 & 76.64932 & 36.65271 & 8.524421 & 29.5879 & 9867.961 & 1.752284 & 1079.011 & 1.30499 \\
\hline Salix repens & 7.5326 & 77.381 & 579.7095 & 436.508 & 112.8081 & 296.6001 & 91.07623 & 107.6241 & 55.66006 & 29.73477 & 6.451181 & 14.33949 & 5524.67 & 1.521649 & 396.7583 & 1.877603 \\
\hline Salix triandra & 4.038336 & 6808.941 & 300.9861 & 839.4107 & 126.8231 & 174.5321 & 61.4648 & 73.9947 & 68.47845 & 29.03301 & 6.615821 & 19.52545 & 7818.192 & 1.159086 & 424.22 & 1.217506 \\
\hline Salix viminalis & 4.301787 & 5999.32 & 362.0131 & 670.0449 & 121.199 & 150.9654 & 63.82114 & 72.92391 & 67.10876 & 23.91047 & 6.45574 & 20.31338 & 5646.995 & 1.453297 & 388.815 & 1.235921 \\
\hline
\end{tabular}




Salvia pratensis
Sambucus nigra
Sambucus nigra cv. 'Laciniata' L.
Sambucus racemosa
Samolus valerandi
Sanguisorba minor
Sanguisorba officinalis
Sanicula europaea
Saponaria officinalis
Saxifraga granulata
Saxifraga tridactylites
Scabiosa columbaria
Scandix pecten-veneris
Schoenoplectus lacustris
Schoenoplectus lacustris ag. (incl. S.
tabernaemontanii
Schoenoplectus tabernaemontani
Schoenus nigricans
Scilla non-scripta
Scirpus sylvaticus
Scleranthus annuus
Scleranthus perennis
Scleranthus polycarpos
Scrophularia auriculata
Scrophularia nodosa
Scrophularia umbrosa
Scrophularia umbrosa s. neesii
Scutellaria galericulata
Scutellaria minor
Sedum acre
Sedum album
Sedum reflexum
Sedum sexangulare
Sedum telephium
Sedum telephium s. telephium
Selinum carvifolia
Senecio aquaticus
Senecio erucifolius
Senecio fluviatilis
Senecio jacobaeaa
Senecio jacobaea s. dunensis
Senecio jacobaea s. jacobaea
Senecio ovatus
Senecio paludosus
Senecio sylvaticus
Senecio viscosus
Senecio vulgariis

\begin{tabular}{|c|c|c|c|c|c|c|c|c|c|c|c|c|c|c|c|}
\hline 8688 & 3954.463 & 49.623 & 292.5055 & 81.08637 & 66.47982 & 34.12554 & 38.91746 & 25.84183 & 11.74467 & 2.616742 & 8.532521 & 2148.898 & 0.947302 & 273.804 & 0.398519 \\
\hline .150362 & 3256.458 & 65.08272 & 715.2192 & 71.6413 & 110.6245 & 42.46169 & 55.19094 & 35.17496 & 14.72226 & 9.612169 & 8786 & 3315.054 & 6748 & 430.0282 & 77 \\
\hline .845888 & 3267.027 & 45.66323 & 610.4516 & 68.08189 & 116.8399 & 38.7831 & 51.94484 & 34.55622 & 14.99136 & 9.387323 & 23.19797 & 2910.569 & 2.674961 & 322.6025 & 18 \\
\hline .663045 & 2800.191 & 44.65298 & 498.5166 & 66.90915 & 103.6435 & 50.3816 & 65.3726 & 36.88516 & 16.73374 & 9.79862 & 31.14204 & 2376.189 & 2.48492 & 298.6854 & 1.405864 \\
\hline .851275 & 6697.555 & 598.6449 & 426.9188 & 113.7816 & 175.0596 & 30.91065 & 58.24833 & 28.15967 & 16.3087 & 4.336102 & 15.82663 & 4723.698 & 1.021208 & 302.0019 & 1.140289 \\
\hline .033121 & 4712.629 & 40.35885 & 354.8481 & 105.7534 & 107.3394 & 56.67731 & 49.99378 & 28.83174 & 18.07639 & 3.697127 & 10.79765 & 2904.346 & 0.585214 & 362.3948 & 0.534025 \\
\hline 486384 & 6996.509 & 139.5659 & 73.5977 & 132.6303 & 228.4252 & 42.36356 & 48.14578 & 34.43563 & 24.61716 & 7.342879 & 14.24018 & 5511.163 & 0.911614 & 784.6988 & 1.017588 \\
\hline .760627 & 2289.688 & 34.60428 & 250.7821 & 43.5252 & 60.89314 & 32.79941 & 23.86908 & 25.92227 & 9.144014 & 3.152106 & 16.82576 & 1387.936 & 1.718459 & 126.883 & 0.693559 \\
\hline .856338 & 3948.03 & 98.08289 & 554.7065 & 100.9163 & 108.0078 & 45.56305 & 54.21951 & 47.07451 & 16.06609 & 6.623229 & 33.6139 & 2930.435 & 1.678964 & 424.3701 & 1.13637 \\
\hline 5.542207 & 6558.066 & 82.34598 & 464.8553 & 142.0197 & 289.4582 & 36.9626 & 42.93626 & 23.4549 & 24.32179 & 7.813352 & 7.046144 & 6024.04 & 0.676777 & 929.9275 & 1.01897 \\
\hline 1.263409 & 2181.691 & 37.32958 & 117.8296 & 44.72104 & 87.70856 & 53.12702 & 49.79667 & 31.64174 & 11.74789 & 3.787473 & 8.071973 & 1442.226 & 1.73154 & 236.0691 & 0.61805 \\
\hline 1.423014 & 3594.919 & 23.84817 & 270.6433 & 74.16872 & 92.18024 & 53.87856 & 49.39357 & 28.3553 & 10.89642 & 4.113174 & 10.21609 & 2072.556 & 0.510856 & 254.0873 & 0.413945 \\
\hline 3.04303 & 3324.518 & 98.81489 & 790.5246 & 135.2427 & 73.15032 & 26.64661 & 26.8395 & 47.98296 & 9.332109 & 3.043109 & 8.150536 & 1657.87 & 1.304953 & 290.6191 & 0.48871 \\
\hline 3.766805 & 5199.52 & 377.3646 & 459.1023 & 90.66931 & 187.4212 & 29.39574 & 42.30231 & 32.44106 & 22.793 & 11.09044 & 26.35344 & 5990.92 & 1.527682 & 573.58 & 1.357497 \\
\hline 3.500571 & 5167.765 & 347.2696 & 287.1956 & 89.00612 & 169.1007 & 27.30585 & 38.96122 & 26.04369 & 21.68149 & 10.9676 & 24.13788 & 5583.52 & 1.333933 & 568.2055 & 1.408777 \\
\hline 6.163111 & 5629.02 & 498.8305 & 477.0395 & 119.703 & 162.5931 & 35.70622 & 59.57678 & 34.26335 & 16.00106 & 5.61878 & 19.5167 & 5251.575 & 1.411258 & 540.9279 & 1.962543 \\
\hline 4.760468 & 6680.58 & 634.3179 & 319.0259 & 114.0912 & 282.5003 & 73.64047 & 80.47569 & 41.71474 & 19.88866 & 4.464465 & 10.36431 & 4369.719 & 1.030886 & 309.8697 & 1.412243 \\
\hline 5.467723 & 2873.276 & 34.55368 & 446.1443 & 63.40342 & 92.99888 & 31.21513 & 46.53231 & 16.74252 & 11.47013 & 8.475899 & 23.74209 & 1480.487 & 2.582373 & 261.6683 & 1.434246 \\
\hline 5.849615 & 8022.015 & 132.6995 & 476.0635 & 121.9334 & 190.8765 & 48.83186 & 64.07159 & 32.666691 & 27.22527 & 8.796764 & 28.47629 & 6354.721 & 1.85865 & 610.9633 & 1.052635 \\
\hline 3.57682 & 3407.009 & 72.34061 & 357.5465 & 91.49322 & 109.236 & 33.97942 & 47.60056 & 39.70843 & 11.39642 & 2.849504 & 11.43729 & 2315.069 & 3.560344 & 474.2577 & 0.947557 \\
\hline 3.917702 & 2797.457 & 22.76341 & 132.6414 & 69.20752 & 89.80747 & 47.42903 & 54.65312 & 32.45596 & 14.24794 & 3.215569 & 8.248967 & 1969.089 & 0.524403 & 263.9765 & 0.882817 \\
\hline 4.777163 & 2572.523 & 17.76183 & 127.9762 & 60.05335 & 72.73236 & 32.31068 & 39.23494 & 40.43505 & 10.95197 & 2.816158 & 7.114472 & 1739.133 & 1.337529 & 225.0384 & 0.836728 \\
\hline 4.957543 & 7824.058 & 252.8761 & 395.768 & 110.461 & 282.0784 & 75.94398 & 99.82547 & 53.87959 & 32.83129 & 7.380576 & 18.10377 & 7572.301 & 0.805476 & 481.6322 & 1.459094 \\
\hline 5.647496 & 3882.619 & 73.44997 & 560.9777 & 77.68027 & 133.5266 & 44.91492 & 51.56743 & 45.558 & 17.3872 & 7.935297 & 21.81221 & 2934.716 & 2.320249 & 249.929 & 1.192804 \\
\hline 5.241855 & 8586.087 & 201.9961 & 808.5458 & 140.7916 & 268.4149 & 82.53605 & 106.8955 & 65.86683 & 31.68191 & 11.99728 & 21.21036 & 8293.295 & 1.656726 & 743.7829 & 2.261514 \\
\hline .371877 & 8351.432 & 261.2899 & 700.8719 & 134.6215 & 277.6292 & 78.4059 & 77.04151 & 76.24092 & 24.79719 & 10.45239 & 18.81558 & 5357.687 & 0.93068 & 699.3756 & 1.425936 \\
\hline 4.898232 & 8184.806 & 190.3375 & 487.2363 & 130.5156 & 244.7037 & 64.20636 & 82.8104 & 45.91966 & 27.57697 & 9.058401 & 25.09082 & 5198.99 & 1.091653 & 557.549 & 1.03666 \\
\hline 5.389191 & 7802.021 & 68.65818 & 327.2467 & 55.42401 & 188.6423 & 53.43463 & 79.36262 & 43.36674 & 27.77325 & 7.773498 & 15.18563 & 4038.065 & 1.063562 & 359.1052 & 0.718014 \\
\hline 1.774901 & 3155.097 & 566.585 & 153.7117 & 67.99345 & 108.9039 & 68.53107 & 61.03932 & 29.73067 & 13.60941 & 3.609077 & 11.44454 & 1771.325 & 1.521116 & 295.1383 & 0.901668 \\
\hline 2.440653 & 4225.986 & 50.60149 & 247.965 & 133.7624 & 125.4054 & 65.25778 & 59.98524 & 47.03427 & 18.63124 & 3.723322 & 27.76927 & 2844.725 & 1.353138 & 463.5486 & 0.849034 \\
\hline 433313 & 3191.734 & 27.19471 & 72.6664 & 77.96323 & 95.51344 & 37.41786 & 49.73423 & 33.00508 & 14.51728 & 3.77493 & 11.32376 & 1944.467 & 0.949031 & 256.3032 & 1.184918 \\
\hline 1.854315 & 3584.905 & 36.11328 & 73.8475 & 91.32814 & 102.6417 & 43.42008 & 48.59605 & 33.30872 & 13.61904 & 3.83345 & 11.65328 & 1704.846 & 1.051711 & 259.644 & 0.867591 \\
\hline 9.625456 & 4146.589 & 55.35708 & 08.2994 & 92.40032 & 106.8659 & 40.90797 & 50.27411 & 43.70194 & 16.70465 & 8.111502 & 33.23427 & 3573.172 & 2.319203 & 398.769 & 2.1344 \\
\hline 7.777676 & 3951.505 & 67.42859 & 506.2147 & 84.67629 & 96.26762 & 39.00832 & 50.88073 & 45.70974 & 16.51985 & 7.006157 & 28.69311 & 3147.502 & 1.675697 & 337.271 & 1.305946 \\
\hline 5.271894 & 9540.472 & 75.87853 & 509.4829 & 121.9086 & 344.4632 & 85.96232 & 119.3986 & 45.21829 & 33.24751 & 8.735128 & 19.70689 & 7355.182 & 1.953722 & 304.6268 & 1.018143 \\
\hline 3.40054 & 4811.525 & 364.2453 & 360.1354 & 108.5176 & 147.096 & 24.56992 & 30.80068 & 26.64683 & 18.21318 & 4.767222 & 27.13618 & 4068.834 & 0.846374 & 552.2169 & 0.657769 \\
\hline 3.718615 & 3999.939 & 400.4876 & 53.4555 & 71.90174 & 95.00361 & 39.34818 & 43.25188 & 36.25545 & 16.49798 & 4.482179 & 14.10886 & 2895.549 & 0.737091 & 268.5288 & 0.518963 \\
\hline 4.405921 & 6879.582 & 177.3085 & 95.4488 & 144.9978 & 175.645 & 58.09405 & 69.89932 & 61.5629 & 21.27922 & 5.878544 & 17.82147 & 5153.133 & 0.9092 & 364.137 & 1.055634 \\
\hline 5.557368 & 4284.25 & 372.9231 & 313.9652 & 108.5332 & 141.0511 & 70.35048 & 66.95282 & 41.73055 & 17.42592 & 5.870987 & 17.8399 & 2515.224 & 1.694285 & 372.0997 & 1.233167 \\
\hline 4.366105 & 2647.102 & 67.08415 & 274.6744 & 67.49042 & 109.3287 & 59.27869 & 52.27935 & 29.99038 & 12.06873 & 6.641753 & 19.79995 & 1805.204 & 1.674373 & 304.4236 & 1.000497 \\
\hline 3.394819 & 5049.915 & 267.5209 & 319.5705 & 111.4584 & 117.5337 & 45.09647 & 50.59069 & 28.23654 & 14.55905 & 4.770682 & .24107 & 2830.613 & 1.302441 & 374.5934 & 0.781877 \\
\hline 4.567488 & 2619.598 & 36.96617 & 337.3327 & 52.76603 & 73.48743 & 31.73379 & 27.96371 & 26.34682 & 8.82284 & 6.707155 & 17.36446 & 1257.794 & 1.380544 & 134.3148 & 0.981352 \\
\hline 3.512922 & 6653.381 & 232.1345 & 507.7597 & 101.211 & 163.9915 & 41.19995 & 55.33592 & 37.65917 & 21.98371 & 6.416692 & 24.90845 & 6490.742 & 1.089096 & 389.4872 & 876731 \\
\hline 9.146822 & 2551.649 & 186.3206 & 624.5953 & 69.9484 & 4.7125 & 60.2015 & 66.15058 & 38.79902 & 13.30185 & 10.0529 & 23.31109 & 2527.178 & 2.112356 & 421.5779 & 2.420958 \\
\hline 2725 & 333 & 149.63 & 598.186 & 1523 & 9112 & 3046 & 99.27815 & 60.52916 & 18.48744 & 3.288582 & 27.19755 & 3190.365 & 2.185089 & 588.5013 & 2.526854 \\
\hline 46346 & 5.589 & 5.5238 & .8087 & 7.0322 & 3666 & 56178 & 3.10298 & .86485 & .92798 & 543193 & .97865 & 865.319 & 2.201184 & 32.0941 & 546 \\
\hline
\end{tabular}




\begin{tabular}{|c|c|c|c|c|c|c|c|c|c|c|c|c|c|c|c|c|}
\hline Seriphidium maritimum & 1.805759 & 5833.459 & 611.2712 & 404.7632 & 121.1977 & 215.4849 & 28.48676 & 33.90274 & 29.42144 & 23.94331 & 4.117577 & 28.44291 & 2624.996 & 1.219995 & 404.0965 & 0.795954 \\
\hline Sherardia arvensis & 2.18073 & 2510.856 & 103.7751 & 501.3889 & 96.98786 & 78.44339 & 26.12231 & 27.48419 & 26.22672 & 11.55261 & 3.006464 & 7.906309 & 1961.888 & 3.119771 & 337.0741 & 0.53239 \\
\hline Silaum silaus & 2.875096 & 6675.666 & 411.4632 & 392.6555 & 127.9563 & 254.9871 & 40.76357 & 50.37783 & 25.80006 & 32.17058 & 3.953601 & 8.394083 & 8762.911 & 0.522932 & 865.191 & 0.517261 \\
\hline Silene conica & 1.166914 & 2757.52 & 35.819 & 83.41396 & 65.90179 & 98.30773 & 59.20847 & 54.92579 & 35.87675 & 12.41458 & 3.094487 & 9.335319 & 1555.168 & 1.68116 & 259.2614 & 0.662156 \\
\hline Silene dioica & 5.541044 & 3403.881 & 50.35907 & 726.342 & 69.45343 & 98.71764 & 36.52324 & 44.5564 & 34.08747 & 13.72705 & 8.106183 & 20.48666 & 2826.128 & 2.178939 & 321.7857 & 1.197656 \\
\hline Silene latifolia & 5.695843 & 2970.291 & 97.44576 & 591.8286 & 78.99755 & 80.61801 & 30.109 & 35.11102 & 39.58287 & 12.13358 & 4.995532 & 21.2222 & 2627.596 & 1.886018 & 353.6428 & 1.246097 \\
\hline Silene nutans & 2.385294 & 2178.248 & 34.02807 & 210.0644 & 68.32742 & 84.48876 & 48.54208 & 40.2471 & 25.17745 & 11.54934 & 4.66697 & 8.415776 & 1552.118 & 1.309197 & 221.3574 & 0.748973 \\
\hline Silene otites & 1.396644 & 2670.117 & 36.5773 & 109.0738 & 67.61323 & 98.5136 & 55.61261 & 48.76796 & 24.24227 & 12.639 & 3.521305 & 7.548773 & 1575.806 & 1.184044 & 234.5984 & 0.612125 \\
\hline Silene vulgaris & 1.318763 & 3699.117 & 69.61533 & 484.4219 & 104.254 & 83.38824 & 51.86147 & 39.01737 & 35.96145 & 15.40403 & 5.552028 & 14.46762 & 2838.068 & 0.568135 & 380.8125 & 0.554099 \\
\hline Sinapis arvensis & 4.163445 & 5569.534 & 135.572 & 702.5216 & 127.465 & 122.2334 & 30.52742 & 35.91869 & 44.99721 & 20.70831 & 4.701686 & 24.3851 & 3562.908 & 2.404772 & 678.638 & 0.702882 \\
\hline Sisymbrium altissimum & 2.604128 & 3952.644 & 161.9311 & 298.0976 & 146.3579 & 125.238 & 68.84994 & 62.92006 & 30.68666 & 14.05516 & 2.923528 & 16.96699 & 2351.324 & 1.330421 & 495.9818 & 0.747182 \\
\hline Sisymbrium officinale & 5.173333 & 3647.539 & 112.0526 & 616.9253 & 120.6476 & 96.73959 & 30.51097 & 36.34123 & 41.49552 & 13.59543 & 4.232775 & 20.50605 & 2657.899 & 1.818609 & 392.496 & 0.983853 \\
\hline Sium latifolium & 3.535375 & 5228.821 & 224.7799 & 403.2472 & 89.39159 & 182.2047 & 29.79854 & 43.28069 & 28.94985 & 20.74869 & 10.43309 & 23.7348 & 5619.239 & 1.115566 & 554.2479 & 0.867676 \\
\hline Solanum dulcamara & 5.291341 & 6676.417 & 297.9006 & 646.2383 & 139.1088 & 304.5362 & 72.0999 & 90.45555 & 64.13222 & 32.98527 & 11.17712 & 19.45441 & 8926.661 & 1.183399 & 923.3991 & 1.681471 \\
\hline Solanum nigrum & 9.363981 & 5762.121 & 359.47 & 746.2759 & 171.9992 & 157.9928 & 61.57082 & 94.2003 & 49.28199 & 16.95149 & 4.880741 & 21.70202 & 3639.522 & 1.888472 & 549.1525 & 2.516182 \\
\hline Solanum nigrum s. nigrum & 9.141577 & 5945.582 & 360.2629 & 757.0755 & 184.8862 & 162.7969 & 59.90477 & 85.76619 & 63.81733 & 21.81658 & 7.75175 & 21.76016 & 4358.785 & 2.356925 & 463.2778 & 2.565982 \\
\hline Solidago virgaurea & 7.946742 & 3355.914 & 44.9415 & 914.2549 & 73.65349 & 101.8648 & 45.03841 & 65.56672 & 47.23928 & 13.18104 & 14.27073 & 31.42501 & 2473.464 & 2.855074 & 393.6629 & 2.191565 \\
\hline Sonchus arvensis & 4.89864 & 5702.506 & 666.5582 & 566.2715 & 161.2973 & 294.6815 & 67.86636 & 69.19353 & 66.97491 & 25.14695 & 6.867012 & 24.64123 & 4325.188 & 2.040348 & 525.7744 & 0.924423 \\
\hline Sonchus arvensis $v$. arvensis & 3.847382 & 4655.003 & 630.0911 & 612.8339 & 133.9916 & 167.1454 & 48.59545 & 53.84978 & 54.88697 & 23.3277 & 7.259902 & 22.97566 & 4754.77 & 1.846671 & 552.4321 & 1.04106 \\
\hline Sonchus arvensis v. maritimus & 2.608074 & 5417.764 & 663.1986 & 375.7176 & 185.9469 & 335.1662 & 78.75638 & 75.5315 & 61.99795 & 22.39068 & 6.060097 & 24.67485 & 2702.096 & 1.751664 & 461.4574 & 0.790315 \\
\hline Sonchus asper & 5.492787 & 5378.248 & 498.0201 & 653.2645 & 139.6488 & 145.5669 & 44.43149 & 54.00489 & 58.04146 & 23.28583 & 6.374425 & 19.5059 & 4152.568 & 1.990047 & 548.5741 & 1.048171 \\
\hline Sonchus oleraceus & 5.552072 & 5333.584 & 610.2913 & 655.0595 & 133.7714 & 146.3383 & 43.53866 & 54.35242 & 57.72483 & 21.26757 & 5.79789 & 21.71801 & 4056.386 & 2.033837 & 466.1345 & 1.204351 \\
\hline Sonchus palustris & 3.359103 & 7317.202 & 228.6732 & 604.6896 & 97.28691 & 210.6659 & 46.52131 & 59.39193 & 40.38272 & 22.7606 & 8.906551 & 21.18713 & 5486.107 & 0.762267 & 398.9529 & 1.324563 \\
\hline Sorbus aucuparia & 6.787836 & 3078.267 & 49.14705 & 549.1515 & 73.13207 & 116.149 & 61.33139 & 83.12336 & 44.03868 & 17.58151 & 9.767079 & 31.34629 & 3038.78 & 2.478878 & 429.1105 & 1.435459 \\
\hline Sparganium emersum & 3.458898 & 4578.419 & 193.3977 & 362.5695 & 83.85235 & 183.1046 & 24.25368 & 26.75478 & 32.71926 & 17.93866 & 11.62845 & 18.04092 & 4953.338 & 1.211026 & 527.3711 & 0.736703 \\
\hline Sparganium erectum & 5.054658 & 6822.842 & 182.1055 & 432.3425 & 113.579 & 185.1221 & 33.24698 & 46.47859 & 29.03064 & 23.15139 & 10.7081 & 24.55795 & 5804.992 & 1.486039 & 568.5631 & 1.133809 \\
\hline Sparganium erectum s. erectum & 4.525292 & 6315.314 & 217.5483 & 463.2647 & 104.6051 & 197.4132 & 31.01678 & 41.41849 & 31.83537 & 19.26549 & 8.733164 & 26.23171 & 5288.193 & 1.381182 & 441.6493 & 0.898063 \\
\hline Spergula arvensis & 4.991292 & 4269.012 & 88.20474 & 518.1244 & 109.1541 & 121.387 & 34.71186 & 49.88505 & 46.36554 & 13.94811 & 3.417864 & 14.05602 & 2972.031 & 2.732879 & 499.7243 & 1.016374 \\
\hline Spergula morisonii & 5.747724 & 848.3835 & 17.89511 & 675.3502 & 28.18927 & 23.26725 & 48.35658 & 67.88303 & 38.36376 & 6.715434 & 5.235901 & 3.869098 & 969.3534 & 0.369914 & 171.9129 & 0.752291 \\
\hline Spergularia marina & 2.08296 & 5317.304 & 593.6104 & 594.1762 & 77.74064 & 132.2283 & 24.11794 & 25.29584 & 38.94996 & 19.64373 & 4.960001 & 15.03618 & 3437.592 & 1.649618 & 350.6713 & 0.819154 \\
\hline Spergularia media s. angustata & 2.335637 & 5747.119 & 574.1256 & 662.6363 & 68.6759 & 159.8996 & 25.92947 & 26.83542 & 32.67147 & 20.96368 & 4.775704 & 15.54732 & 2881.235 & 1.74853 & 383.0327 & 0.712174 \\
\hline Spergularia rubra & 6.613191 & 4231.415 & 106.6312 & 368.7435 & 114.2315 & 135.1338 & 70.77906 & 101.167 & 60.03227 & 19.17708 & 4.057633 & 13.43416 & 3849.357 & 3.484566 & 495.4341 & 1.371737 \\
\hline Spirodela polyrhiza & 3.473784 & 4971.622 & 199.1828 & 368.1147 & 76.61782 & 177.3589 & 19.15936 & 24.86358 & 23.56705 & 17.95243 & 7.686872 & 25.374 & 4640.506 & 1.162573 & 398.7119 & 0.691598 \\
\hline Stachys arvensis & 2.855328 & 2747.062 & 99.39203 & 539.8117 & 80.61844 & 90.51418 & 26.40685 & 33.32433 & 37.12567 & 10.46148 & 2.761164 & 14.64415 & 2280.394 & 1.684999 & 268.6191 & 0.779638 \\
\hline Stachys officinalis & 6.570324 & 3160.251 & 17.54566 & 443.5088 & 60.91499 & 80.17661 & 31.76856 & 31.79578 & 29.14503 & 8.808961 & 5.15562 & 13.41985 & 2532.901 & 0.492017 & 234.8688 & 2.224075 \\
\hline Stachys palustris & 4.256174 & 7457.83 & 195.4938 & 466.4228 & 120.4849 & 242.3557 & 52.36657 & 67.03598 & 45.4801 & 31.58995 & 11.10544 & 20.77887 & 7185.491 & 0.997501 & 528.2189 & 1.089571 \\
\hline Stachys sylvatica & 4.312567 & 3104.626 & 53.05234 & 353.0753 & 58.37322 & 94.01887 & 39.55985 & 37.49537 & 36.18201 & 13.08734 & 5.332034 & 15.31108 & 2501.037 & 0.982572 & 162.4269 & 0.849571 \\
\hline Stellaria aquatica & 5.51958 & 7669.191 & 245.8105 & 646.3419 & 146.3476 & 167.3441 & 59.97955 & 83.96484 & 61.91121 & 32.9184 & 7.445471 & 20.19491 & 7860.521 & 1.557928 & 449.962 & 1.39779 \\
\hline Stellaria graminea & 5.079206 & 8189.594 & 103.8474 & 466.5514 & 109.9356 & 165.2977 & 50.44875 & 66.91265 & 36.76455 & 29.69642 & 6.495818 & 14.85504 & 5580.686 & 1.474754 & 417.4636 & 1.017644 \\
\hline Stellaria holostea & 5.558191 & 3338.454 & 37.42015 & 706.5069 & 67.37204 & 93.55215 & 37.1222 & 50.58479 & 31.3529 & 12.21255 & 10.68395 & 23.15051 & 1810.705 & 2.687301 & 193.5637 & 1.347239 \\
\hline Stellaria media & 9.084023 & 6328.874 & 105.4552 & 716.8006 & 157.0447 & 153.4935 & 57.43301 & 84.30684 & 45.78312 & 21.11101 & 9.147704 & 22.04972 & 4829.693 & 2.065625 & 579.6632 & 1.812168 \\
\hline Stellaria media + S. pallida + S. neglecta & 7.16787 & 2687.873 & 116.4099 & 331.4944 & 86.0697 & 121.5059 & 57.72502 & 63.29283 & 40.21272 & 10.74714 & 7.624396 & 32.11991 & 1888.194 & 2.029707 & 291.3917 & 1.034629 \\
\hline Stellaria pallida & 4.672623 & 3521.227 & 249.876 & 315.5007 & 79.85512 & 323.2261 & 118.2006 & 139.0727 & 79.30956 & 41.56591 & 7.235994 & 28.09992 & 7173.506 & 1.234765 & 434.439 & 1.120154 \\
\hline Stellaria palustris & 3.573614 & 4449.932 & 192.8813 & 371.8986 & 104.168 & 192.6063 & 28.79173 & 38.6066 & 29.24189 & 19.10786 & 8.163197 & 22.84742 & 3502.743 & 1.006711 & 585.6547 & 0.690034 \\
\hline Stellaria uliginosa & 5.838572 & 7943.809 & 137.0747 & 468.2216 & 136.9251 & 191.417 & 47.45092 & 64.56939 & 33.73909 & 25.46752 & 7.777111 & 24.89942 & 6184.684 & 1.700441 & 501.9581 & 1.117777 \\
\hline Stratiotes aloides & 3.235892 & 5081.95 & 162.5452 & 517.8279 & 78.9322 & 142.1412 & 25.21723 & 39.33696 & 20.02357 & 19.45731 & .29064 & 23.03041 & 115.803 & 1.309937 & 6.8231 & 708 \\
\hline
\end{tabular}




\begin{tabular}{|c|c|c|c|c|c|c|c|c|c|c|c|c|c|c|c|c|}
\hline Succisa pratensis & 5.06143 & 6207.207 & 65.84086 & 498.7377 & 73.28565 & 211.8623 & 54.32315 & 63.96956 & 30.43191 & 24.27664 & 5.874955 & 10.18981 & 5300.528 & 0.913307 & 343.6752 & 1.105565 \\
\hline Symphytum officinale & 5.430133 & 8136.616 & 174.8147 & 746.7668 & 122.1749 & 192.2936 & 51.32315 & 62.19622 & 55.63652 & 27.59558 & 8.576022 & 24.17873 & 5398.703 & 1.073413 & 365.372 & 1.134248 \\
\hline Tanacetum vulgare & 6.132093 & 3856.729 & 118.1101 & 530.0126 & 101.1069 & 104.7381 & 34.33434 & 44.76551 & 44.42046 & 14.67342 & 6.051277 & 20.6838 & 3088.718 & 1.748393 & 370.3612 & 1.29541 \\
\hline Taraxacum commixtum & 1.132812 & 2906.91 & 33.02691 & 132.4041 & 75.35908 & 90.47746 & 62.03518 & 86.48337 & 40.47359 & 15.63023 & 4.041299 & 9.814819 & 1798.659 & 1.641123 & 271.0988 & 0.932089 \\
\hline Taraxacum lacistophyllum & 4.557188 & 2362.366 & 37.72894 & 202.8717 & 67.71621 & 91.34616 & 51.30372 & 49.33254 & 43.70196 & 11.7412 & 3.577463 & 13.34155 & 1719.96 & 1.82588 & 259.4202 & 1.714084 \\
\hline Taraxacum nordstedtii & 2.763468 & 7746.596 & 61.67943 & 452.4274 & 75.69185 & 138.8267 & 44.74296 & 57.44995 & 22.16055 & 24.55686 & 4.44583 & 7.414856 & 5960.868 & 0.752367 & 569.7568 & 0.529113 \\
\hline Taraxacum obliquum & 2.168177 & 2099.858 & 100.122 & 171.1283 & 55.93922 & 83.54372 & 46.61439 & 36.41845 & 25.8829 & 10.48869 & 4.542595 & 7.317146 & 1183.067 & 1.728885 & 172.4781 & 0.968749 \\
\hline Taraxacum rubicundum & 2.48628 & 2575.385 & 29.82767 & 180.1364 & 58.86143 & 97.82744 & 51.27106 & 47.62199 & 31.33295 & 13.79494 & 4.342283 & 10.03927 & 1701.267 & 1.617931 & 248.0478 & 0.980185 \\
\hline Taraxacum sectie Celtica & 6.510099 & 9792.485 & 176.436 & 447.8553 & 189.8887 & 304.835 & 111.8855 & 132.5225 & 80.40776 & 40.65062 & 7.944669 & 16.02989 & 7810.753 & 1.226656 & 653.1645 & 2.231087 \\
\hline Taraxacum sectie Erythrosperma & 3.064156 & 2600.43 & 41.02745 & 214.7099 & 70.81053 & 96.99988 & 51.68369 & 46.20579 & 26.39306 & 10.16891 & 5.516002 & 11.31757 & 1643.088 & 1.702588 & 250.1986 & 1.041172 \\
\hline Taraxacum sectie Hamata & 6.187659 & 5715.336 & 72.24526 & 494.2933 & 106.481 & 120.2276 & 40.8735 & 52.44687 & 33.6029 & 19.73913 & 6.491263 & 15.8235 & 3863.753 & 1.331647 & 384.8018 & 1.258673 \\
\hline Taraxacum sectie Palustria & 3.483011 & 6860.549 & 673.9645 & 414.4407 & 112.6864 & 199.979 & 39.15525 & 49.08415 & 24.72475 & 25.38187 & 6.028399 & 11.42713 & 6104.629 & 0.696831 & 550.5864 & 1.038429 \\
\hline Taraxacum sectie Ruderalia & 7.257669 & 6985.804 & 306.7701 & 559.9368 & 152.5711 & 145.1943 & 51.05998 & 62.62615 & 49.37118 & 23.45327 & 6.00328 & 19.13488 & 5586.876 & 1.379771 & 642.53 & 1.428702 \\
\hline Taraxacum taeniatum & 2.916276 & 1655.061 & 23.61403 & 197.1396 & 50.77115 & 61.28707 & 46.85404 & 41.63701 & 44.35481 & 9.027877 & 5.80111 & 11.31069 & 1407.164 & 1.948608 & 253.2013 & 1.401828 \\
\hline Taraxacum tortilobum & 2.723978 & 3055.432 & 35.93345 & 172.3915 & 84.36939 & 97.39003 & 47.94797 & 51.01934 & 30.71862 & 13.35529 & 4.061228 & 13.45522 & 2018.829 & 1.686052 & 296.7165 & 1.257607 \\
\hline Taxus baccata & 6.52369 & 2567.623 & 30.44747 & 574.1079 & 60.96853 & 87.90087 & 39.66052 & 58.73398 & 23.40323 & 14.71442 & 9.837934 & 28.59431 & 2016.284 & 2.59197 & 463.6238 & 1.471084 \\
\hline Teesdalia nudicaulis & 4.888622 & 2561.246 & 29.43921 & 228.0027 & 54.71602 & 72.35294 & 68.29117 & 72.57472 & 35.00694 & 10.87563 & 3.288807 & 11.30837 & 1903.998 & 2.675371 & 334.9221 & 0.968494 \\
\hline Tephroseris palustris & 4.965148 & 8853.558 & 609.1867 & 973.0362 & 143.2594 & 276.4333 & 52.69203 & 73.22392 & 55.68032 & 33.62801 & 11.15574 & 25.333 & 9465.545 & 1.213219 & 715.2776 & 0.934945 \\
\hline Teucrium scordium & 1.797123 & 3012.702 & 60.3997 & 261.6841 & 46.13115 & 82.34039 & 18.08386 & 18.80615 & 13.51674 & 9.800925 & 2.979298 & 6.522987 & 2401.116 & 0.885946 & 198.6269 & 0.499377 \\
\hline Teucrium scorodonia & 7.713404 & 2550.814 & 40.90748 & 980.476 & 58.35605 & 91.32607 & 40.79091 & 61.52217 & 26.96556 & 13.90699 & 12.46008 & 26.53636 & 2768.071 & 2.751552 & 486.8694 & 1.892016 \\
\hline Thalictrum flavum & 3.774977 & 6907.705 & 155.2391 & 523.4673 & 123.2926 & 148.8738 & 42.10972 & 46.11288 & 32.31854 & 21.58325 & 6.467681 & 29.28186 & 4253.554 & 1.318334 & 442.3124 & 0.922026 \\
\hline Thalictrum minus & 3.117485 & 3768.853 & 44.28033 & 345.5412 & 74.85128 & 72.53888 & 38.29293 & 45.30579 & 40.67341 & 14.32408 & 4.460582 & 21.16961 & 2331.264 & 1.243644 & 367.0971 & 0.551555 \\
\hline Thalictrum minus s. dunense & 4.607318 & 2917.474 & 20.47742 & 178.5969 & 65.02559 & 90.61228 & 52.42302 & 36.64485 & 42.44913 & 15.24188 & 5.047879 & 15.70706 & 1938.462 & 2.49885 & 285.0015 & 1.817535 \\
\hline Thelypteris palustris & 4.479499 & 7396.62 & 147.9043 & 390.1365 & 107.4812 & 202.21 & 53.76386 & 71.95303 & 31.2248 & 23.06897 & 7.26424 & 30.97841 & 3688.228 & 1.239242 & 690.9742 & 0.859451 \\
\hline Thlaspi arvense & 3.041576 & 3626.348 & 85.63552 & 904.2219 & 116.5796 & 107.0356 & 24.32598 & 28.23372 & 44.02616 & 13.58236 & 3.961307 & 17.83373 & 2918.732 & 1.65408 & 278.2231 & 0.606887 \\
\hline Thymus pulegioides & 2.907451 & 3029.049 & 38.93659 & 211.5719 & 80.51792 & 89.4624 & 51.40478 & 47.66065 & 27.0343 & 13.29784 & 4.22944 & 10.0487 & 1746.86 & 1.007671 & 249.786 & 0.901463 \\
\hline Thymus serpyllum & 6.502515 & 3733.004 & 30.91839 & 202.8418 & 87.14204 & 109.0783 & 56.10061 & 71.82788 & 33.06497 & 17.94968 & 4.124892 & 10.53497 & 2365.027 & 0.474358 & 298.6542 & 1.668832 \\
\hline Tilia cordata & 4.211219 & 2739.128 & 29.45918 & 493.8494 & 54.1578 & 76.85142 & 32.38313 & 36.37924 & 25.45465 & 9.224013 & 7.133954 & 19.92458 & 1073.042 & 1.779086 & 240.1227 & 1.012399 \\
\hline Tilia platyphyllos & 5.766095 & 2693.224 & 28.01272 & 496.0903 & 60.64361 & 83.6356 & 31.5027 & 37.04269 & 18.55415 & 9.052032 & 11.80406 & 20.54729 & 1315.098 & 2.926072 & 176.2203 & 1.357278 \\
\hline Torilis japonica & 7.094739 & 3754.022 & 61.07299 & 631.5876 & 84.95102 & 90.66207 & 37.01635 & 40.8499 & 39.33158 & 14.2308 & 6.388725 & 23.587 & 2750.315 & 1.605041 & 366.4281 & 1.290996 \\
\hline Torilis nodosa & 1.577877 & 4048.915 & 205.0557 & 465.9683 & 110.2001 & 100.9899 & 24.59872 & 27.22214 & 23.27767 & 9.640661 & 2.989338 & 8.346437 & 2168.137 & 1.435748 & 273.7892 & 0.683656 \\
\hline Tragopogon porrifolius & 1.470175 & 2539.434 & 110.839 & 264.1659 & 48.78375 & 45.28025 & 13.089 & 14.15191 & 13.23482 & 5.611928 & 1.401233 & 5.498803 & 1217.778 & 0.264563 & 110.4077 & 0.201237 \\
\hline Tragopogon pratensis & 2.246545 & 5203.006 & 61.80303 & 384.1987 & 107.1335 & 89.42003 & 43.0988 & 47.41027 & 31.50329 & 17.42941 & 3.83479 & 10.60588 & 3526.53 & 0.958507 & 407.7988 & 0.530407 \\
\hline Tragopogon pratensis s. minor & 3.030672 & 6285.043 & 88.99936 & 258.2301 & 136.124 & 138.6376 & 84.84209 & 109.8775 & 48.54471 & 20.43626 & 5.796242 & 22.00851 & 2655.326 & 1.226726 & 412.9513 & 0.912442 \\
\hline Tragopogon pratensis s. orientalis & 1.598312 & 7939.629 & 71.64977 & 465.6507 & 89.88294 & 74.18342 & 54.83797 & 41.3693 & 23.47037 & 13.29583 & 4.003676 & 11.12651 & 2884.837 & 0.724696 & 284.3891 & 0.784155 \\
\hline Tragopogon pratensis s. pratensis & 2.506485 & 3968.669 & 61.88653 & 403.5135 & 73.11985 & 74.17997 & 30.14001 & 31.99157 & 19.71133 & 10.56002 & 2.674059 & 8.047463 & 2492.188 & 0.74588 & 248.2248 & 0.557846 \\
\hline Trichophorum cespitosum & 6.638455 & 3193.648 & 17.12225 & 799.0751 & 62.87043 & 116.189 & 83.27655 & 131.1687 & 32.85748 & 22.53423 & 6.643324 & 5.659097 & 3607.851 & 2.178834 & 249.4477 & 0.752887 \\
\hline Trichophorum cespitosum s. germanicum & 6.576933 & 2718.903 & 20.9405 & 671.1647 & 57.57751 & 86.09369 & 77.1002 & 131.689 & 47.71362 & 16.89731 & 7.445146 & 6.217912 & 3296.192 & 2.622498 & 282.0855 & 0.728836 \\
\hline Trifolium arvense & 3.959722 & 3836.668 & 139.8473 & 197.6191 & 106.423 & 119.6299 & 52.59709 & 56.12623 & 30.02964 & 14.53828 & 3.503692 & 11.09566 & 2031.969 & 1.630089 & 297.2051 & 1.28575 \\
\hline Trifolium campestre & 3.018383 & 4313.479 & 379.7677 & 210.0361 & 119.1622 & 127.5065 & 52.59683 & 58.93435 & 33.88565 & 16.51671 & 3.753317 & 11.14533 & 2432.726 & 1.422649 & 335.2136 & 0.948067 \\
\hline Trifolium dubium & 3.300971 & 7095.083 & 378.7934 & 360.6469 & 120.305 & 133.177 & 45.95438 & 55.74089 & 30.71581 & 21.35057 & 4.67466 & 11.24989 & 4203.691 & 1.150463 & 382.9167 & 0.925944 \\
\hline Trifolium fragiferum & 2.573518 & 6395.609 & 600.125 & 422.8363 & 95.2246 & 157.5909 & 31.82567 & 32.4766 & 38.58017 & 15.27381 & 4.917279 & 10.13427 & 4094.748 & 0.923244 & 398.0436 & 0.8401 \\
\hline Trifolium hybridum & 4.524807 & 8118.21 & 475.9887 & 457.5779 & 142.247 & 197.3984 & 49.46642 & 58.38534 & 38.75546 & 24.18276 & 6.976911 & 13.04308 & 5246.995 & 1.140712 & 416.8983 & 1.238812 \\
\hline Trifolium medium & 4.407665 & 6253.754 & 84.18598 & 542.6593 & 149.1004 & 137.568 & 47.64893 & 60.50675 & 31.61082 & 22.26925 & 6.374529 & 15.004 & 6276.473 & 0.608637 & 474.011 & 1.334209 \\
\hline Trifolium micranthum & 3.400666 & 6090.606 & 113.5208 & 232.5151 & 131.6802 & 175.4182 & 72.01425 & 54.79566 & 42.45273 & 21.14683 & 4.469159 & 10.51419 & 3751.1 & 0.908345 & 343.2776 & 1.00007 \\
\hline Trifolium pratense & 3.332277 & 6414.707 & 511.0239 & 404.1768 & 107.8669 & 124.6701 & 36.90465 & 44.10993 & 29.31925 & 20.23259 & 15242 & 11.50871 & 144.426 & 836554 & 83.7427 & 0.94271 \\
\hline
\end{tabular}




\begin{tabular}{|c|c|c|c|c|c|c|c|c|c|c|c|c|c|c|c|c|}
\hline Trifolium repens & 3.254025 & 6664.899 & 643.0943 & 477.9062 & 135.3473 & 144.0983 & 38.42825 & 46.60629 & 35.49002 & 20.88526 & 4.940408 & 12.76262 & 4965.593 & 1.021923 & 523.2134 & 1.019374 \\
\hline Trifolium scabrum & 1.491159 & 3462.074 & 718.7662 & 109.558 & 90.6446 & 104.6537 & 54.61397 & 62.84418 & 41.30552 & 13.84573 & 3.177006 & 10.79525 & 1904.936 & 0.955647 & 266.8663 & 0.955811 \\
\hline Trifolium striatum & 2.355333 & 3466.767 & 46.79708 & 153.8606 & 91.12939 & 103.2402 & 41.23228 & 51.22195 & 30.62781 & 11.56312 & 3.437905 & 9.204666 & 1821.607 & 1.35013 & 240.2172 & 0.801122 \\
\hline Triglochin maritima & 2.973662 & 6136.223 & 558.2993 & 399.3271 & 87.51071 & 165.8065 & 31.40715 & 29.68055 & 40.41345 & 14.12068 & 5.91387 & 12.03523 & 3962.665 & 0.747858 & 272.5702 & 0.833557 \\
\hline Triglochin palustris & 4.473063 & 4997.134 & 664.4905 & 353.7825 & 100.8655 & 141.8986 & 29.59667 & 30.99655 & 34.39782 & 16.79505 & 5.689561 & 12.11865 & 6770.813 & 0.985968 & 612.3369 & 1.671224 \\
\hline Tripleurospermum maritimum & 3.942115 & 5165.738 & 614.1559 & 582.1624 & 147.0522 & 163.2114 & 38.05632 & 45.13333 & 46.23687 & 21.12438 & 5.425922 & 19.22268 & 4086.849 & 2.360656 & 543.2847 & 1.025224 \\
\hline Trisetum flavescens & 2.411378 & 4280.801 & 62.8529 & 424.0279 & 88.26703 & 85.49368 & 36.69346 & 38.96089 & 24.33712 & 12.97814 & 2.671038 & 8.092377 & 2999.545 & 0.742222 & 315.7041 & 0.592689 \\
\hline Tuberaria guttata & 3.974909 & 2223.851 & 32.12474 & 122.4859 & 48.52268 & 86.2466 & 57.4949 & 81.23342 & 48.67663 & 12.44383 & 3.43473 & 5.498372 & 1637.763 & 0.71786 & 219.9309 & 0.585047 \\
\hline Tussilago farfara & 5.013996 & 4315.362 & 472.6246 & 772.1454 & 121.0188 & 143.0036 & 40.95674 & 50.07597 & 47.05364 & 19.08073 & 7.324038 & 23.39539 & 3828.717 & 1.953963 & 379.875 & 1.134166 \\
\hline Typha angustifolia & 4.233589 & 6599.234 & 277.8049 & 397.7742 & 121.3367 & 182.0872 & 36.50549 & 55.1179 & 31.19713 & 24.09958 & 9.772979 & 28.22918 & 6088.427 & 1.319168 & 653.7583 & 1.299374 \\
\hline Typha latifolia & 5.145128 & 7712.375 & 331.9622 & 481.593 & 125.3801 & 214.7728 & 41.32164 & 58.1178 & 39.99241 & 27.75853 & 11.60661 & 31.10827 & 6705.565 & 1.769786 & 649.5532 & 1.669787 \\
\hline Ulmus glabra & 5.827482 & 3072.567 & 46.44298 & 593.5603 & 63.07471 & 83.99353 & 54.63747 & 44.09593 & 22.21986 & 10.68201 & 8.660932 & 19.00672 & 2015.21 & 3.486879 & 277.8813 & 1.600037 \\
\hline Ulmus minor & 5.387172 & 2856.621 & 41.73674 & 732.0323 & 60.29517 & 71.6728 & 34.01978 & 32.49327 & 19.67841 & 9.698363 & 5.740231 & 16.10291 & 1476.225 & 2.217017 & 203.9188 & 1.722425 \\
\hline Urtica dioica & 6.925964 & 4988.034 & 154.4162 & 873.5579 & 113.6843 & 176.0539 & 54.76766 & 67.70069 & 53.05411 & 26.39783 & 9.384757 & 23.77217 & 5643.031 & 1.795511 & 577.5618 & 1.782511 \\
\hline Urtica urens & 6.797261 & 5105.966 & 224.8927 & 712.4469 & 177.133 & 135.7688 & 42.06312 & 52.07284 & 39.77026 & 14.04689 & 3.761937 & 17.68017 & 3733.098 & 1.782809 & 652.8895 & 1.44407 \\
\hline Utricularia minor & 8.013762 & 6445.578 & 172.6858 & 378.3802 & 134.624 & 146.1273 & 26.51439 & 41.11166 & 24.76359 & 23.54068 & 8.766176 & 26.90376 & 3420.973 & 2.146175 & 590.9577 & 0.848117 \\
\hline Utricularia vulgaris & 4.299478 & 5132.434 & 149.6762 & 623.4115 & 95.83579 & 158.8702 & 26.07165 & 39.67247 & 28.71268 & 21.1714 & 10.31032 & 30.27346 & 5337.929 & 1.549475 & 541.9384 & 0.989962 \\
\hline Vaccinium myrtillus & 5.363413 & 1367.96 & 32.69199 & 491.9607 & 44.23552 & 68.14571 & 49.27 & 61.08305 & 42.62671 & 13.00321 & 9.724547 & 13.18208 & 2035.492 & 2.125031 & 396.6878 & 0.823501 \\
\hline Vaccinium uliginosum & 4.522791 & 3054.082 & 38.56211 & 1061.564 & 68.10606 & 130.7873 & 104.993 & 141.2733 & 39.42075 & 20.39372 & 11.4746 & 7.182981 & 3548.867 & 2.168327 & 332.1087 & 1.331172 \\
\hline Vaccinium vitis-idaea & 5.192393 & 1606.394 & 32.7139 & 568.4991 & 52.6763 & 79.89795 & 63.99659 & 73.87228 & 54.2575 & 14.3379 & 9.665012 & 8.870905 & 2805.262 & 2.051545 & 323.3923 & 0.963035 \\
\hline Valeriana dioica & 4.098848 & 6441.025 & 103.8422 & 407.8074 & 71.28225 & 184.1703 & 36.5052 & 44.27861 & 25.0903 & 18.13937 & 5.794015 & 21.73447 & 2890.66 & 1.247444 & 407.1724 & 0.557632 \\
\hline Valeriana officinalis & 5.256085 & 8773.623 & 176.8418 & 591.0097 & 119.352 & 227.5329 & 62.23556 & 74.10443 & 57.67605 & 29.882 & 7.238455 & 25.98084 & 6345.565 & 1.309353 & 552.5817 & 1.19563 \\
\hline Valerianella dentata & 2.416345 & 7661.329 & 70.48924 & 505.2094 & 121.1221 & 178.7918 & 62.54054 & 53.74784 & 60.55046 & 40.116 & 2.900814 & 14.29277 & 7946.927 & 2.535282 & 233.5078 & 1.027567 \\
\hline Valerianella locusta & 2.393049 & 5179.39 & 87.76025 & 406.9123 & 136.9959 & 111.0877 & 61.65869 & 60.16491 & 31.56957 & 20.86022 & 3.626302 & 13.27576 & 3368.493 & 1.789761 & 433.0681 & 0.600933 \\
\hline Verbascum densiflorum & 7.235445 & 3337.293 & 128.4311 & 717.7645 & 136.3721 & 109.2292 & 39.60961 & 50.94791 & 43.83631 & 11.34221 & 6.265896 & 32.73027 & 3932.08 & 1.894184 & 726.7549 & 1.433136 \\
\hline Verbascum nigrum & 5.681701 & 3432.283 & 82.15125 & 625.7187 & 88.7621 & 80.81612 & 35.06687 & 39.98782 & 40.72859 & 11.57483 & 5.758896 & 32.92389 & 2465.561 & 1.676925 & 363.3777 & 1.091464 \\
\hline Verbascum thapsus & 6.054657 & 3233.097 & 70.36151 & 398.3602 & 103.9167 & 123.7448 & 50.09009 & 54.13031 & 33.72368 & 14.57257 & 6.480719 & 32.50005 & 2271.543 & 1.957439 & 391.2404 & 1.129095 \\
\hline Verbena officinalis & 3.883796 & 5135.244 & 268.696 & 550.285 & 120.2576 & 132.4628 & 43.08108 & 49.34329 & 42.27628 & 15.7965 & 4.808327 & 21.92903 & 3462.951 & 1.200579 & 522.8456 & 0.836483 \\
\hline Veronica agrestis & 3.229033 & 6264.19 & 104.4766 & 578.942 & 182.2936 & 151.9268 & 32.86401 & 39.30955 & 40.10004 & 16.23639 & 3.556689 & 15.40964 & 3775.826 & 1.798813 & 728.1703 & 0.692004 \\
\hline Veronica anagallis-aquatica & 2.901049 & 8126.507 & 318.623 & 861.1847 & 118.6209 & 231.4177 & 45.17905 & 64.64999 & 46.60623 & 31.79978 & 6.203212 & 21.21879 & 8410.898 & 1.325935 & 593.3212 & 0.970459 \\
\hline Veronica arvensis & 3.344286 & 5317.371 & 61.32999 & 293.7443 & 128.924 & 134.0995 & 71.22921 & 67.21824 & 35.71967 & 16.55475 & 4.328209 & 11.85833 & 2901.728 & 2.522701 & 425.4553 & 1.005086 \\
\hline Veronica austriaca s. teucrium & 1.635278 & 3128.473 & 48.68064 & 243.3629 & 69.96936 & 56.78553 & 31.72859 & 33.27124 & 31.28261 & 10.95576 & 2.331316 & 8.138467 & 1647.453 & 1.034385 & 241.633 & 0.50145 \\
\hline Veronica beccabunga & 4.960826 & 6524.174 & 224.2196 & 433.3823 & 106.8314 & 237.3328 & 44.59187 & 62.25856 & 44.24173 & 25.99309 & 6.589595 & 21.89928 & 6747.818 & 1.161929 & 546.6697 & 1.178673 \\
\hline Veronica catenata & 2.854239 & 7108.136 & 351.3008 & 488.689 & 101.4507 & 205.5948 & 38.8494 & 49.05838 & 44.41647 & 28.85858 & 5.286512 & 14.53216 & 7261.623 & 0.910259 & 551.8831 & 0.852637 \\
\hline Veronica chamaedrys & 7.844106 & 5839.082 & 55.37235 & 619.0358 & 110.3203 & 120.5949 & 54.85348 & 62.55296 & 48.65396 & 23.89177 & 5.69689 & 25.53018 & 4162.375 & 1.390189 & 443.25 & 1.446201 \\
\hline Veronica filiformis & 6.011855 & 7547.722 & 151.3321 & 556.9399 & 147.2061 & 187.3244 & 62.10538 & 74.87622 & 57.21635 & 24.86361 & 6.457981 & 19.53626 & 5036.261 & 1.526342 & 479.5622 & 1.479967 \\
\hline Veronica hederifolia & 7.286126 & 2989.002 & 63.02788 & 1091.037 & 72.38174 & 78.90885 & 37.59293 & 39.45124 & 18.81787 & 12.29293 & 7.92026 & 28.34662 & 2293.057 & 1.642084 & 351.4036 & 1.366199 \\
\hline Veronica hederifolia s. hederifolia & 6.986735 & 4461.513 & 84.31231 & 1039.896 & 133.5331 & 104.3664 & 44.49788 & 40.50782 & 20.93672 & 18.17223 & 7.246414 & 29.13245 & 2949.16 & 3.146438 & 382.8151 & 1.245996 \\
\hline Veronica longifolia & 3.028036 & 8170.677 & 213.6403 & 530.3878 & 143.2123 & 310.2222 & 51.54222 & 65.94552 & 37.2896 & 26.85855 & 5.558504 & 27.20866 & 8101.187 & 1.019538 & 998.6314 & 0.541375 \\
\hline Veronica montana & 3.592048 & 3470.492 & 48.91521 & 244.94 & 61.75392 & 86.33159 & 35.42562 & 28.98255 & 31.97062 & 13.78829 & 4.165685 & 15.5354 & 1868.018 & 0.904641 & 144.0917 & 0.795309 \\
\hline Veronica officinalis & 7.811313 & 2564.552 & 39.17932 & 311.6406 & 69.51442 & 94.32318 & 50.75786 & 49.9335 & 34.47199 & 12.30551 & 6.966946 & 12.68277 & 2004.555 & 1.852932 & 282.8829 & 1.780819 \\
\hline Veronica persica & 3.178327 & 3384.026 & 92.0644 & 825.3682 & 118.6058 & 83.92814 & 25.6938 & 28.55114 & 39.37154 & 11.50735 & 3.96264 & 12.936 & 2679.971 & 1.420439 & 487.0672 & 0.543504 \\
\hline Veronica prostrata & 2.074567 & 2717.591 & 33.60568 & 131.5666 & 72.83927 & 79.15058 & 32.83406 & 43.30836 & 27.05174 & 9.567328 & 2.56126 & 11.71765 & 1536.996 & 1.204609 & 233.322 & 0.834693 \\
\hline Veronica scutellata & 3.921255 & 5302.952 & 229.9211 & 407.9645 & 117.1533 & 203.9299 & 30.68465 & 47.322 & 30.92718 & 20.4049 & 7.678879 & 18.69833 & 3941.899 & 1.254266 & 722.0784 & 0.7179 \\
\hline Veronica serpyllifolia & 4.287729 & 8768.462 & 103.7932 & 499.2005 & 152.807 & 155.29 & 43.40867 & 55.65986 & 31.04278 & 23.4614 & 5.656793 & 13.5762 & 4730.467 & 1.336577 & 460.459 & 0.881625 \\
\hline Viburnum opulus & 4.484415 & 3597.317 & 56.5256 & 384.7869 & 67.2186 & 145.575 & 45.49747 & 49.54014 & 48.27159 & 17.41236 & 6.572117 & 19.07849 & 3523.049 & 1.118304 & 347.9614 & 91933 \\
\hline
\end{tabular}




\begin{tabular}{|c|c|c|c|c|c|c|c|c|c|c|c|c|c|c|c|c|}
\hline Vicia cracca & 5.122057 & 7982.829 & 237.6602 & 479.4818 & 112.7897 & 162.8538 & 46.84533 & 54.72723 & 39.3773 & 28.1832 & 6.18021 & 17.92106 & 5620.711 & 1.118745 & 433.7791 & 119098 \\
\hline Vicia hirsuta & 4.248034 & 4477.223 & 81.78727 & 457.1305 & 91.97141 & 104.5184 & 37.83939 & 43.88933 & 42.9658 & 16.89002 & 4.033957 & 12.10807 & 2780.799 & 3.87191 & 401.9756 & 0.958049 \\
\hline Vicia lathyroides & 2.780826 & 2725.81 & 61.09457 & 200.6566 & 74.75703 & 97.40287 & 58.29411 & 50.32014 & 32.96619 & 10.73804 & 4.896973 & 10.14956 & 1570.806 & 1.687262 & 263.8694 & 1.037958 \\
\hline Vicia sativa & 5.165344 & 5085.338 & 141.1731 & 485.6874 & 91.7794 & 101.1519 & 32.35531 & 40.83888 & 41.36353 & 18.75449 & 5.37517 & 16.24421 & 3159.859 & 2.267386 & 420.445 & 1.20476 \\
\hline Vicia sativa s. nigra & 4.419814 & 4351.309 & 119.619 & 347.6643 & 118.8631 & 109.9472 & 57.24803 & 59.24003 & 42.55949 & 13.94677 & 3.923416 & 11.94024 & 2445.778 & 3.909553 & 408.8429 & 1.088176 \\
\hline Vicia sativa s. sativa & 4.354182 & 4002.699 & 107.4177 & 444.9679 & 97.19544 & 90.54854 & 30.22419 & 36.11733 & 30.37302 & 20.43933 & 4.925639 & 13.08883 & 3579.773 & 2.155407 & 389.8062 & 1.092186 \\
\hline Vicia sepium & 8.601419 & 5144.562 & 62.63693 & 705.4494 & 83.38657 & 92.12685 & 57.6603 & 54.95616 & 47.51291 & 17.99187 & 6.802707 & 27.41659 & 3258.958 & 1.325902 & 265.1474 & 1.425176 \\
\hline Vicia tetrasperma & 3.271859 & 4043.579 & 527.133 & 539.4134 & 89.57448 & 105.8365 & 28.41535 & 35.81694 & 35.76187 & 14.56783 & 4.923263 & 15.50194 & 2870.381 & 1.8111 & 366.0729 & 0.808187 \\
\hline Vicia tetrasperma s. tetrasperma & 4.215557 & 5337.32 & 92.37458 & 459.3359 & 96.99867 & 113.1416 & 34.97253 & 45.69604 & 27.48342 & 20.49639 & 5.554694 & 12.56989 & 3779.941 & 0.9561 & 311.9785 & 0.905855 \\
\hline Vinca minor & 4.163872 & 2579.227 & 34.61683 & 530.7217 & 50.31774 & 78.92207 & 36.66701 & 35.75737 & 27.92386 & 9.057712 & 6.357232 & 20.59692 & 1510.078 & 1.718178 & 134.9046 & 1.013472 \\
\hline Viola arvensis & 4.313582 & 3803.03 & 73.38229 & 518.3427 & 109.2234 & 108.6224 & 32.30143 & 43.36446 & 37.51445 & 11.09767 & 2.959109 & 13.01355 & 2376.983 & 2.572695 & 449.3208 & 1.057008 \\
\hline Viola canina & 7.061247 & 3601.358 & 43.29899 & 286.8492 & 76.81353 & 130.1873 & 81.64331 & 85.67198 & 42.54929 & 28.81655 & 6.715853 & 9.886239 & 3021.251 & 0.911748 & 354.5739 & 1.397876 \\
\hline Viola curtisii & 2.985054 & 2075.658 & 39.34967 & 205.7006 & 48.60788 & 92.31387 & 53.17841 & 46.81157 & 27.01415 & 11.59877 & 4.67323 & 12.47922 & 1515.56 & 1.634468 & 265.6663 & 1.148201 \\
\hline Viola hirta & 7.035366 & 2772.508 & 50.80909 & 439.2354 & 65.8382 & 90.96414 & 41.27895 & 40.5791 & 40.02584 & 13.37415 & 5.179249 & 29.45855 & 2001.795 & 1.546006 & 296.2768 & 1.094695 \\
\hline Viola odorata & 6.005168 & 2815.379 & 37.62174 & 1030.782 & 81.09804 & 90.83959 & 41.80974 & 35.79345 & 20.44884 & 14.49143 & 5.958017 & 22.58223 & 3351.67 & 1.312763 & 566.5543 & 1.555508 \\
\hline Viola palustris & 4.755084 & 7168.514 & 139.3743 & 469.243 & 73.61121 & 183.587 & 43.30186 & 56.02396 & 26.48784 & 23.04817 & 8.435722 & 22.94958 & 3070.07 & 1.878057 & 404.7754 & 0.725429 \\
\hline Viola persicifolia & 4.044605 & 6781.214 & 83.69042 & 406.8743 & 62.01369 & 80.17057 & 39.42469 & 31.59883 & 23.6414 & 23.4808 & 4.821764 & 8.016832 & 3749.917 & 0.647343 & 245.7769 & 0.51621 \\
\hline Viola reichenbachiana & 3.13143 & 2520.059 & 33.26443 & 332.2081 & 46.29435 & 65.80307 & 32.22057 & 25.05037 & 24.57099 & 9.540585 & 3.936189 & 15.77401 & 1473.395 & 1.125082 & 135.1429 & 0.797653 \\
\hline Viola reichenbachiana + V. riviniana & 4.633518 & 3148.757 & 39.66661 & 568.5735 & 57.43054 & 101.7134 & 36.43015 & 43.77839 & 39.40136 & 12.50163 & 6.437188 & 28.24537 & 2316.772 & 1.442018 & 311.5435 & 0.924324 \\
\hline Viola riviniana & 5.406789 & 2982.773 & 44.09414 & 641.481 & 61.52005 & 96.44846 & 33.60582 & 38.46456 & 36.22187 & 14.74177 & 6.240708 & 29.96151 & 2820.819 & 1.546505 & 366.5341 & 1.172173 \\
\hline Viola rupestris & 3.690679 & 2099.355 & 31.82561 & 177.549 & 59.85031 & 78.34719 & 46.53631 & 40.91301 & 24.19595 & 9.904706 & 3.682317 & 6.886225 & 1415.495 & 1.214325 & 196.667 & 0.736284 \\
\hline Viola tricolor & 3.015505 & 2896.719 & 53.38571 & 192.011 & 65.94124 & 104.9328 & 69.22599 & 65.04701 & 34.73487 & 13.22701 & 3.309639 & 14.66109 & 1857.364 & 2.420897 & 291.3152 & 1.576298 \\
\hline Vulpia bromoides & 5.415885 & 5469.521 & 62.46765 & 231.672 & 137.594 & 126.6489 & 51.57555 & 62.9067 & 32.70849 & 25.22424 & 3.632737 & 11.46455 & 2932.161 & 1.023308 & 320.1348 & 1.97517 \\
\hline Vulpia myuros & 5.523213 & 3815.832 & 50.31858 & 257.7707 & 96.72557 & 117.1147 & 48.92154 & 65.89133 & 37.97047 & 13.86501 & 2.239174 & 14.42064 & 2032.139 & 2.726647 & 311.2538 & 2.161438 \\
\hline average & 4.798073 & 5053.526 & 204.5368 & 504.577 & 99.37894 & 150.3108 & 47.76734 & 57.08995 & 39.21138 & 19.15289 & 6.389447 & 18.3713 & 3824.64 & 1.533952 & 416.5902 & 1.111747 \\
\hline s.e. & 2.005692 & 1985.65 & 201.4795 & 207.1762 & 31.43604 & 63.70408 & 17.16842 & 23.14781 & 12.22295 & 7.05273 & 2.601063 & 7.412545 & 1786.691 & 0.690171 & 165.3977 & 0.41239 \\
\hline
\end{tabular}




\begin{tabular}{|c|c|c|c|c|c|c|c|c|c|c|c|c|c|c|c|c|}
\hline & $\begin{array}{c}\mathbf{C} / \mathbf{N} \\
-\end{array}$ & $\begin{array}{c}\mathrm{Ca}(\mathrm{H} 20) \\
\mathrm{mg} / \mathrm{kg}\end{array}$ & $\begin{array}{c}\mathrm{Cl} \\
\mathrm{mg} / \mathrm{kg}\end{array}$ & $\begin{array}{l}\text { Electronic conductivity } \\
\mathrm{mS}\end{array}$ & $\begin{array}{c}\mathrm{K}(\mathrm{H} 20) \\
\mathrm{mg} / \mathrm{kg}\end{array}$ & $\begin{array}{c}\mathrm{Mg}(\mathrm{H} 20) \\
\mathrm{mg} / \mathrm{kg}\end{array}$ & $\begin{array}{l}\text { MHL } \\
\mathrm{cm}-\mathrm{s}\end{array}$ & $\begin{array}{l}\text { MLL } \\
\mathrm{cm}-\mathrm{s}\end{array}$ & $\begin{array}{l}\text { MSL } \\
\text { cm -s }\end{array}$ & $\begin{array}{c}\text { moist } \\
\%\end{array}$ & $\begin{array}{c}\mathrm{NH4} \\
(\mathrm{CaCL2}) \\
\mathrm{mg} / \mathrm{kg}\end{array}$ & $\begin{array}{c}\mathrm{NO3} \\
(\mathrm{COCL} 2) \\
\mathrm{mg} / \mathrm{kg}\end{array}$ & $\begin{array}{l}\mathrm{N} \text { total } \\
\mathrm{mg} / \mathrm{kg}\end{array}$ & $\begin{array}{c}\mathbf{P O 4} \\
(\mathrm{CaCl}) \\
\mathrm{mg} / \mathrm{kg} \\
\end{array}$ & $\begin{array}{l}\mathbf{P} \text { total } \\
\mathrm{mg} / \mathrm{kg}\end{array}$ & pH \\
\hline Agrostis capillaris & 9.846809 & 5700.484 & 69.93728 & 668.8926 & 125.1467 & 163.236 & 72.57885 & 99.92051 & 48.03353 & 26.91459 & 10.7731 & 17.47814 & 4993.225 & 3.233671 & 484.5942 & 1.840516 \\
\hline Agrostis gigantea & 5.917801 & 5988.828 & 192.9489 & 599.5446 & 130.4806 & 238.1821 & 60.41447 & 74.25869 & 54.21917 & 32.07277 & 9.494162 & 26.76384 & 8411.405 & 1.172523 & 638.5303 & 1.432781 \\
\hline Alliaria petiolata & 4.935723 & 3075.666 & 60.75243 & 661.6413 & 66.25094 & 79.82954 & 33.93016 & 34.34582 & 20.28321 & 11.37803 & 5.312887 & 15.99306 & 2381.434 & 1.064361 & 365.5297 & 1.271545 \\
\hline Alnus glutinosa & 5.637878 & 5896.373 & 135.1159 & 561.7079 & 89.23107 & 314.2781 & 72.66927 & 91.96947 & 62.85787 & 22.51251 & 10.02754 & 24.80724 & 4075.25 & 1.969467 & 504.1171 & 1.144964 \\
\hline Ammophila arenaria & 4.032584 & 2268.641 & 527.5923 & 212.5634 & 59.41648 & 99.43565 & 60.19599 & 68.03287 & 31.33833 & 11.30267 & 4.509116 & 13.30597 & 1699.967 & 1.42225 & 292.9166 & 1.84412 \\
\hline Asparagus officinalis & 5.18164 & 3789.951 & 488.9529 & 500.2494 & 99.03109 & 214.9835 & 80.83609 & 85.83608 & 67.70228 & 19.07457 & 7.883896 & 29.52333 & 2551.767 & 1.490062 & 338.6893 & 1.8077 \\
\hline Bellis perennis & 2.153817 & 5706.261 & 430.357 & 455.2241 & 108.985 & 100.7773 & 29.60499 & 35.82925 & 24.83869 & 16.72687 & 3.755223 & 9.275012 & 4121.982 & 0.730607 & 418.0048 & 0.667419 \\
\hline Calluna vulgaris & 8.885939 & 2012.993 & 23.42489 & 1106.002 & 63.63132 & 92.47385 & 75.81665 & 95.73338 & 50.10261 & 15.90058 & 10.38143 & 7.33103 & 3197.32 & 2.173929 & 457.4038 & 1.228182 \\
\hline Cardamine flexuosa & 5.159174 & 5741.695 & 101.3516 & 628.9488 & 86.70585 & 209.8881 & 54.2321 & 65.05433 & 50.73987 & 25.28274 & 11.40387 & 19.63261 & 4138.493 & 1.165691 & 369.243 & 1.0043 \\
\hline Carduus nutans & 2.114806 & 4857.54 & 125.5173 & 359.4998 & 122.5323 & 109.2031 & 37.33329 & 40.08366 & 27.30191 & 16.27646 & 3.792969 & 14.42704 & 2917.36 & 1.662128 & 419.7555 & 0.569552 \\
\hline Cerastium fontanum & 6.884967 & 8376.981 & 299.7579 & 475.815 & 136.5138 & 156.521 & 54.07372 & 72.68137 & 30.83788 & 26.02973 & 5.935607 & 15.32327 & 5045.469 & 1.346355 & 476.8201 & 1.687406 \\
\hline Cirsium arvense & 5.566974 & 5699.537 & 517.8702 & 559.6947 & 144.3042 & 145.2082 & 45.61681 & 53.97261 & 44.48545 & 23.06763 & 6.96683 & 21.0806 & 5082.137 & 1.363936 & 575.2049 & 1.130069 \\
\hline Cirsium vulgare & 6.397181 & 5396.19 & 502.8083 & 647.9096 & 142.7731 & 146.7396 & 51.45536 & 59.67995 & 47.38912 & 19.89546 & 7.508797 & 24.91363 & 4023.279 & 1.639467 & 469.8361 & 1.387125 \\
\hline Cynodon dactylon & 1.727899 & 4235.539 & 109.6552 & 238.1644 & 104.028 & 103.9647 & 41.49608 & 43.90367 & 30.41512 & 14.14079 & 2.782733 & 10.25648 & 2457.712 & 1.314721 & 410.7468 & 0.53902 \\
\hline Cynoglossum officinale & 5.625077 & 2659.738 & 239.974 & 351.6373 & 78.93347 & 118.3108 & 50.88293 & 44.14875 & 27.31762 & 12.25991 & 7.490265 & 26.54738 & 1923.427 & 1.003335 & 339.6641 & 0.882381 \\
\hline Erodium cicutarium & 3.938967 & 4089.644 & 75.69764 & 250.4003 & 118.0817 & 132.5035 & 67.9765 & 62.86581 & 37.45392 & 13.63506 & 2.77163 & 16.20517 & 2544.361 & 2.895282 & 475.9611 & 1.167429 \\
\hline Eupatorium cannabinum & 4.975925 & 7946.343 & 407.8965 & 599.0892 & 101.7185 & 249.903 & 59.36162 & 70.48578 & 46.67329 & 24.81247 & 9.297414 & 26.94644 & 5867.062 & 1.130516 & 681.6754 & 1.504479 \\
\hline Euphorbia esula & 4.596667 & 4325.457 & 133.5533 & 536.7034 & 107.7767 & 94.85775 & 37.25364 & 43.11307 & 44.1681 & 15.76347 & 4.795688 & 19.48387 & 3277.433 & 1.231432 & 368.5062 & 0.672096 \\
\hline Fragaria vesca & 6.712801 & 3152.511 & 66.05711 & 716.97 & 68.94891 & 105.9341 & 43.71364 & 44.75841 & 46.38023 & 15.24695 & 6.650346 & 30.36295 & 2774.938 & 1.696322 & 364.1867 & 1.576316 \\
\hline Glyceria maxima & 4.678548 & 6614.918 & 208.9554 & 484.8013 & 109.9673 & 214.4293 & 38.05664 & 53.17953 & 34.36204 & 21.24423 & 9.652238 & 26.52625 & 5942.724 & 1.087198 & 531.5757 & 0.915283 \\
\hline Hieracium pilosella & 6.632947 & 3585.28 & 35.07258 & 238.4453 & 85.46663 & 109.3293 & 61.27998 & 61.73522 & 31.87675 & 15.52272 & 5.481692 & 12.17167 & 2288.858 & 0.942748 & 306.5416 & 1.88918 \\
\hline Holcus lanatus & 8.161333 & 8865.611 & 374.0871 & 581.2583 & 138.0408 & 210.6547 & 62.47026 & 84.09689 & 44.77264 & 31.18185 & 8.680249 & 21.1596 & 6321.474 & 2.293483 & 587.6023 & 1.595473 \\
\hline Hypericum perforatum & 7.276227 & 4584.994 & 49.83298 & 535.8585 & 92.93256 & 126.3281 & 48.67871 & 61.10306 & 41.73053 & 21.32678 & 7.478299 & 18.03197 & 3474.749 & 1.353152 & 352.2903 & 1.790071 \\
\hline Hypochaeris radicata & 7.53174 & 4850.894 & 67.3257 & 295.7497 & 113.9157 & 144.9395 & 69.16182 & 79.48829 & 36.65762 & 22.58637 & 5.231458 & 12.82244 & 3478.171 & 1.017431 & 453.6508 & 1.705862 \\
\hline Iris pseudacorus & 5.415559 & 8112.205 & 205.2399 & 456.0517 & 132.9453 & 257.6129 & 63.65294 & 85.67792 & 46.69551 & 28.11105 & 10.18682 & 25.53604 & 6470.663 & 1.311404 & 649.222 & 1.110656 \\
\hline Ligustrum vulgare & 5.567401 & 2601.739 & 210.8556 & 545.0192 & 77.16553 & 94.85189 & 36.55596 & 41.08676 & 39.33346 & 13.17971 & 5.575243 & 26.90431 & 2145.411 & 0.952525 & 332.4696 & 1.35055 \\
\hline Linaria vulgaris & 6.113371 & 4042.579 & 82.39908 & 449.1101 & 101.4887 & 107.4031 & 46.51797 & 52.54618 & 35.2669 & 18.46076 & 6.431637 & 17.40137 & 3154.443 & 1.965174 & 408.3865 & 1.471949 \\
\hline Lotus corniculatus ag. (incl. L. glaber) & 4.085049 & 6802.642 & 694.3585 & 312.0066 & 142.4492 & 154.1974 & 75.66022 & 76.45576 & 42.1681 & 23.26401 & 4.860073 & 14.72768 & 3691.967 & 1.046605 & 483.0963 & 1.399584 \\
\hline Luzula campestris & 8.55363 & 4550.745 & 52.8278 & 296.6127 & 94.43336 & 140.0294 & 79.91531 & 85.72658 & 43.71506 & 22.28022 & 6.926793 & 11.70867 & 3061.751 & 1.485444 & 375.9862 & 1.899536 \\
\hline Lythrum salicaria & 4.941909 & 7165.752 & 229.8603 & 499.1365 & 115.6332 & 221.6143 & 50.13815 & 72.47492 & 45.51697 & 24.74603 & 9.431885 & 29.50502 & 5455.422 & 1.697613 & 747.0847 & 1.114583 \\
\hline Melilotus albus & 2.989464 & 4945.985 & 412.9599 & 419.2097 & 151.3191 & 126.7205 & 34.81729 & 40.09971 & 40.34503 & 15.23953 & 3.189878 & 17.76015 & 2971.151 & 1.82399 & 394.0341 & 0.717799 \\
\hline Phalaris arundinacea & 7.23037 & 8736.611 & 193.9638 & 511.6349 & 143.1419 & 213.783 & 57.76587 & 81.92184 & 48.67965 & 30.15776 & 8.197203 & 23.46989 & 7712.673 & 1.366598 & 612.5144 & 1.305368 \\
\hline Phragmites australis & 6.649669 & 8352.489 & 612.0805 & 689.0666 & 140.4918 & 260.2474 & 61.31773 & 83.10764 & 50.1183 & 30.03864 & 12.26503 & 33.89231 & 7438.242 & 1.88123 & 857.8412 & 2.152015 \\
\hline Poa annua & 7.092602 & 7429.924 & 515.6447 & 598.3555 & 183.8809 & 153.6167 & 47.3437 & 60.62467 & 46.32957 & 20.297 & 5.774088 & 15.04167 & 4810.65 & 1.758199 & 597.8899 & 1.776923 \\
\hline Poa pratensis & 5.781803 & 6385.691 & 589.3725 & 408.9369 & 154.216 & 168.1123 & 67.9662 & 75.53085 & 41.14223 & 25.28098 & 6.016298 & 15.74836 & 4937.118 & 1.454005 & 509.6029 & 1.397392 \\
\hline Populus alba & 6.982764 & 3645.014 & 187.6172 & 1079.036 & 97.8066 & 138.9576 & 48.43236 & 57.9918 & 30.84858 & 17.938 & 7.972503 & 31.90316 & 3623.128 & 1.654295 & 449.718 & 2.202651 \\
\hline Ranunculus ficaria & 5.705589 & 7132.617 & 106.4626 & 713.7471 & 95.02052 & 126.0332 & 54.97911 & 57.82947 & 27.83245 & 27.52746 & 6.413912 & 19.741 & 4038.007 & 1.56966 & 221.2025 & 1.000727 \\
\hline Rhamnus cathartica & 6.132295 & 5354.503 & 442.8266 & 671.5149 & 87.89926 & 245.5493 & 68.61673 & 74.05767 & 67.90577 & 21.0675 & 8.443267 & 29.29412 & 3764.56 & 1.868047 & 384.183 & 1.69046 \\
\hline Rubus ulmifolius & 5.625807 & 5710.642 & 174.7982 & 787.6532 & 177.7925 & 150.5742 & 42.94052 & 56.92743 & 34.71956 & 16.61123 & 5.053474 & 23.22074 & 3848.876 & 2.757017 & 342.8521 & 2.116069 \\
\hline Rumex acetosella & 10.20791 & 3491.833 & 42.64592 & 541.7114 & 88.41874 & 107.7862 & 70.13978 & 90.18461 & 35.99952 & 16.66975 & 7.013294 & 12.84301 & 2940.04 & 3.004855 & 465.0986 & 1.76183 \\
\hline Rumex crispus & 3.624253 & 8171.961 & 419.7925 & 513.211 & 127.5608 & 135.6747 & 44.98304 & 51.86277 & 45.25484 & 26.77107 & 5.479406 & 13.1496 & 6946.163 & 0.983474 & 578.4639 & 0.988859 \\
\hline Sagina procumbens & 4.114885 & 9464.01 & 578.5852 & 450.1838 & 186.1715 & 237.1128 & 54.62024 & 63.19172 & 49.51423 & 28.47523 & 6.989932 & 15.68226 & 5818.646 & 1.498165 & 498.2362 & 1.39043 \\
\hline Salix cinerea & 5.835026 & 6209.761 & 307.0701 & 620.4355 & 98.55733 & 285.313 & 73.50701 & 100.7294 & 55.66443 & 23.34337 & 10.8555 & 32.85579 & 4588.837 & 1.935497 & 648.2195 & 1.262264 \\
\hline Senecio jacobaea & 5.557368 & 4284.25 & 372.9231 & 313.9652 & 108.5332 & 141.0511 & 70.35048 & 66.95282 & 41.73055 & 17.42592 & 5.870987 & 17.8399 & 2515.224 & 1.694285 & 372.0997 & 1.233167 \\
\hline
\end{tabular}




\begin{tabular}{|c|c|c|c|c|c|c|c|c|c|c|c|c|c|c|c|c|}
\hline Senecio viscosus & 11.2725 & 4538.333 & 149.63 & 598.186 & 148.1523 & 161.9112 & 76.3046 & 99.27815 & 60.52916 & 18.48744 & 3.288582 & 27.19755 & 3190.365 & 2.185089 & 588.5013 & 2.526854 \\
\hline Senecio vulgaris & 5.746346 & 5455.589 & 595.5238 & 520.8087 & 167.0322 & 172.3666 & 75.56178 & 73.10298 & 50.86485 & 19.92798 & 4.543193 & 20.97865 & 3865.319 & 2.201184 & 682.0941 & 1.556466 \\
\hline Stellaria media & 9.084023 & 6328.874 & 105.4552 & 716.8006 & 157.0447 & 153.4935 & 57.43301 & 84.30684 & 45.78312 & 21.11101 & 9.147704 & 22.04972 & 4829.693 & 2.065625 & 579.6632 & 1.812168 \\
\hline Trifolium dubium & 3.300971 & 7095.083 & 378.7934 & 360.6469 & 120.305 & 133.177 & 45.95438 & 55.74089 & 30.71581 & 21.35057 & 4.67466 & 11.24989 & 4203.691 & 1.150463 & 382.9167 & 0.925944 \\
\hline Trifolium repens & 3.254025 & 6664.899 & 643.0943 & 477.9062 & 135.3473 & 144.0983 & 38.42825 & 46.60629 & 35.49002 & 20.88526 & 4.940408 & 12.76262 & 4965.593 & 1.021923 & 523.2134 & 1.019374 \\
\hline Tussilago farfara & 5.013996 & 4315.362 & 472.6246 & 772.1454 & 121.0188 & 143.0036 & 40.95674 & 50.07597 & 47.05364 & 19.08073 & 7.324038 & 23.39539 & 3828.717 & 1.953963 & 379.875 & 1.134166 \\
\hline Verbascum thapsus & 6.054657 & 3233.097 & 70.36151 & 398.3602 & 103.9167 & 123.7448 & 50.09009 & 54.13031 & 33.72368 & 14.57257 & 6.480719 & 32.50005 & 2271.543 & 1.957439 & 391.2404 & 1.129095 \\
\hline Veronica serpyllifolia & 4.287729 & 8768.462 & 103.7932 & 499.2005 & 152.807 & 155.29 & 43.40867 & 55.65986 & 31.04278 & 23.4614 & 5.656793 & 13.5762 & 4730.467 & 1.336577 & 460.459 & 0.881625 \\
\hline Vulpia bromoides & 5.415885 & 5469.521 & 62.46765 & 231.672 & 137.594 & 126.6489 & 51.57555 & 62.9067 & 32.70849 & 25.22424 & 3.632737 & 11.46455 & 2932.161 & 1.023308 & 320.1348 & 1.97517 \\
\hline average & 5.777552 & 5544.948 & 269.0287 & 523.0018 & 117.7916 & 159.6582 & 55.45327 & 65.71821 & 41.86094 & 20.85173 & 6.805538 & 20.07111 & 4108.312 & 1.603844 & 470.1763 & 1.387867 \\
\hline s.e. & 1.980056 & 1893.953 & 198.9737 & 186.4443 & 30.86005 & 55.48452 & 13.76348 & 17.81343 & 10.52407 & 5.342736 & 2.379888 & 7.025855 & 1552.748 & 0.554367 & 128.1833 & 0.44985 \\
\hline
\end{tabular}




\begin{tabular}{|c|c|c|c|c|c|c|c|c|c|c|c|c|c|c|c|c|}
\hline Species name & $\begin{array}{c}\mathrm{C} / \mathrm{N} \\
-\end{array}$ & $\begin{array}{c}\mathrm{Ca}(\mathrm{H} 20) \\
\mathrm{mg} / \mathrm{kg}\end{array}$ & $\begin{array}{c}\mathrm{Cl} \\
\mathrm{mg} / \mathrm{kg}\end{array}$ & $\begin{array}{c}\text { Electronic } \\
\text { conductivity } \\
\mathrm{mS}\end{array}$ & $\begin{array}{c}\mathbf{K}(\mathbf{H} 20) \\
\mathrm{mg} / \mathrm{kg}\end{array}$ & $\begin{array}{c}\mathrm{Mg}(\mathrm{H} 20) \\
\mathrm{mg} / \mathrm{kg}\end{array}$ & $\begin{array}{l}\text { MHL } \\
\text { cm -s }\end{array}$ & $\begin{array}{l}\text { MLL } \\
\text { cm -s }\end{array}$ & $\begin{array}{l}\text { MSL } \\
\text { cm -s }\end{array}$ & $\begin{array}{c}\text { moist } \\
\%\end{array}$ & $\begin{array}{c}\mathrm{NH4} \\
(\mathrm{CaCL2}) \\
\mathrm{mg} / \mathrm{kg}\end{array}$ & $\begin{array}{c}\mathrm{NO3} \\
(\mathrm{CaCL} 2) \\
\mathrm{mg} / \mathrm{kg}\end{array}$ & $\begin{array}{l}\mathrm{N} \text { total } \\
\mathrm{mg} / \mathrm{kg}\end{array}$ & $\begin{array}{c}\mathbf{P O 4} \\
(\mathrm{CaCl}) \\
\mathrm{mg} / \mathrm{kg}\end{array}$ & $\begin{array}{l}\text { P total } \\
\mathbf{m g} / \mathrm{kg}\end{array}$ & $\begin{array}{l}\mathrm{pH} \\
-\end{array}$ \\
\hline Acer campestre & 5.553644 & 3323.07 & 58.2693 & 794.4663 & 66.66616 & 83.23061 & 38.73252 & 38.51169 & 27.278 & 10.63282 & 6.192317 & 20.0749 & 2044.906 & 1.812571 & 293.6717 & 1.709258 \\
\hline Acer pseudoplatanus & 5.939691 & 3052.253 & 42.28228 & 567.2719 & 66.34433 & 100.5146 & 42.83811 & 56.7878 & 25.38807 & 14.41649 & 10.55699 & 25.80448 & 2572.518 & 2.784178 & 440.2174 & 1.454065 \\
\hline Achillea millefolium & 4.526244 & 4726.602 & 71.07194 & 370.232 & 109.482 & 117.145 & 41.61421 & 50.36372 & 31.65867 & 17.15065 & 4.769478 & 14.31548 & 3523.849 & 1.547499 & 398.2702 & 1.279099 \\
\hline Achillea ptarmica & 4.535008 & 7342.648 & 164.8867 & 403.8691 & 121.2373 & 177.1139 & 40.14364 & 44.99661 & 30.82617 & 23.39632 & 6.074322 & 18.32204 & 4510.147 & 1.052815 & 472.1282 & 1.021612 \\
\hline Adoxa moschatellina & 3.287658 & 2701.908 & 41.27066 & 315.654 & 50.54858 & 78.02412 & 38.18353 & 29.93804 & 29.12908 & 12.87618 & 4.183746 & 16.02067 & 2088.851 & 0.862203 & 135.9543 & 0.719121 \\
\hline Aegopodium podagraria & 6.10162 & 3706.445 & 64.09181 & 728.4882 & 73.95944 & 90.23904 & 38.81519 & 43.48735 & 28.43493 & 13.41367 & 6.974243 & 21.71277 & 2396.175 & 1.430624 & 198.4055 & 1.187659 \\
\hline Aethusa cynapium & 3.881247 & 3507.261 & 86.66569 & 745.3573 & 135.5819 & 83.7876 & 32.32471 & 37.20702 & 43.20138 & 11.4013 & 3.919176 & 19.80565 & 2370.34 & 1.546425 & 291.4879 & 0.697776 \\
\hline Agrimonia eupatoria & 3.734833 & 4532.47 & 60.6522 & 521.0123 & 89.9139 & 97.00106 & 39.73775 & 44.27241 & 28.98138 & 15.90561 & 3.709401 & 14.2004 & 3110.67 & 0.665495 & 351.1137 & 0.524096 \\
\hline Agrostis canina & 6.879456 & 9617.248 & 134.1308 & 602.2643 & 136.9248 & 331.0072 & 71.75748 & 104.5859 & 43.18426 & 35.68326 & 11.38887 & 14.28826 & 7660 & 1.929682 & 1049.568 & 1.068945 \\
\hline Agrostis canina ag. (incl. A. vinealis) & 9.181115 & 7836.632 & 81.42574 & 817.6027 & 129.416 & 237.3963 & 123.8988 & 172.7018 & 67.91007 & 42.29991 & 14.49056 & 18.80497 & 808.9232 & 3.492897 & 118.7599 & 1.270761 \\
\hline Agrostis stolonifera & 7.373595 & 8654.556 & 685.0757 & 513.2532 & 137.3677 & 269.8903 & 59.72882 & 79.80272 & 55.3702 & 32.91927 & 8.028078 & 19.88874 & 9381.599 & 1.573761 & 907.8245 & 1.995342 \\
\hline Agrostis vinealis & 8.654843 & 1971.134 & 30.76981 & 905.8192 & 53.42281 & 51.48783 & 64.17541 & 81.69516 & 72.88498 & 10.01233 & 7.565694 & 7.061162 & 2228.212 & 1.018793 & 399.0688 & 1.297753 \\
\hline Aira caryophyllea & 4.791975 & 3285.402 & 27.30597 & 211.3393 & 84.4749 & 104.3367 & 47.18574 & 53.86791 & 31.88306 & 13.8188 & 3.309402 & 9.585471 & 2144.801 & 1.231763 & 274.8699 & 0.998236 \\
\hline Aira praecox & 6.779396 & 2250.058 & 32.8437 & 193.8455 & 49.83318 & 81.75433 & 57.17707 & 58.02215 & 31.43125 & 11.82145 & 3.903406 & 11.08733 & 1782.29 & 1.473976 & 287.4814 & 1.824481 \\
\hline Ajuga reptans & 6.416167 & 9218.204 & 57.3348 & 694.935 & 96.31054 & 174.6696 & 66.38559 & 75.44723 & 50.28471 & 31.46442 & 6.389014 & 28.83635 & 6358.606 & 1.680647 & 494.019 & 1.199106 \\
\hline Alisma lanceolatum & 2.965506 & 4143.867 & 191.8205 & 330.8307 & 64.31442 & 214.816 & 31.84343 & 44.79371 & 37.20504 & 21.74558 & 5.978473 & 15.60224 & 5772.576 & 0.885605 & 344.8429 & 0.778562 \\
\hline Alisma plantago-aquatica & 4.035376 & 5504.222 & 204.3954 & 409.4099 & 98.19089 & 201.3708 & 32.99527 & 44.88435 & 37.30851 & 20.8982 & 10.93516 & 28.40001 & 5230.332 & 1.514224 & 525.6195 & 0.950735 \\
\hline Allium oleraceum & 5.922544 & 3785.79 & 48.0961 & 371.4105 & 78.04263 & 94.2711 & 38.99342 & 42.37737 & 40.98052 & 14.14921 & 5.226552 & 28.86691 & 3096.785 & 1.081228 & 461.0544 & 1.003792 \\
\hline Allium scorodoprasum & 7.76479 & 2470.813 & 33.31511 & 1058.45 & 48.43974 & 69.30454 & 27.73533 & 24.42522 & 17.23954 & 10.64836 & 4.377808 & 16.53118 & 2432.562 & 0.872364 & 353.6148 & 1.330504 \\
\hline Allium ursinum & 3.454485 & 2484.223 & 28.71658 & 639.9525 & 48.37697 & 64.3204 & 26.94602 & 19.93194 & 22.23464 & 10.04266 & 5.158146 & 15.06589 & 1061.293 & 0.889253 & 193.0752 & 0.801023 \\
\hline Allium vineale & 6.737198 & 4815.062 & 68.65175 & 613.4722 & 107.855 & 120.2328 & 39.53333 & 44.93058 & 38.1779 & 16.36824 & 5.426008 & 27.4985 & 3235.747 & 1.179598 & 387.8566 & 1.061935 \\
\hline Alopecurus aequalis & 4.597707 & 6996.266 & 220.5729 & 446.1218 & 123.4497 & 190.4749 & 36.25392 & 50.533 & 38.71231 & 27.53085 & 6.718945 & 22.12153 & 7553.293 & 1.191358 & 628.3343 & 1.161479 \\
\hline Alopecurus bulbosus & 2.178242 & 4317.051 & 608.6053 & 449.0124 & 68.12205 & 118.8016 & 23.1107 & 26.64721 & 29.48927 & 17.78423 & 4.317767 & 9.147256 & 2954.449 & 0.798268 & 291.6229 & 0.933589 \\
\hline Alopecurus geniculatus & 2.734556 & 5213.758 & 440.9543 & 332.7738 & 87.2176 & 141.1182 & 31.65024 & 49.08594 & 37.12234 & 23.59648 & 4.156207 & 9.149609 & 7017.149 & 0.691765 & 377.2957 & 0.721991 \\
\hline Alopecurus myosuroides & 2.348498 & 3113.243 & 82.69111 & 751.5198 & 99.64728 & 83.20326 & 22.87734 & 24.80097 & 40.56239 & 10.95328 & 3.281431 & 10.62846 & 2567.115 & 1.258233 & 241.3249 & 0.475475 \\
\hline Alopecurus pratensis & 5.312341 & 7110.489 & 91.29173 & 510.1124 & 113.2155 & 96.07852 & 35.2163 & 44.83377 & 48.04288 & 24.36045 & 4.598115 & 16.64041 & 3753.711 & 0.76054 & 303.9979 & 0.664844 \\
\hline Althaea officinalis & 3.656425 & 5553.65 & 596.1748 & 752.2998 & 98.31757 & 157.8961 & 33.37147 & 39.94334 & 32.74152 & 17.53929 & 6.146283 & 17.39199 & 3993.133 & 0.978752 & 264.1862 & 0.937889 \\
\hline Anagallis arvensis & 2.525799 & 4503.157 & 108.9576 & 677.9739 & 155.9315 & 114.0621 & 38.11684 & 42.58138 & 42.44886 & 12.89345 & 3.337189 & 15.5589 & 2751.919 & 2.025583 & 531.078 & 0.745244 \\
\hline Anagallis arvensis s. arvensis & 3.492352 & 4604.372 & 638.2115 & 552.2331 & 163.8482 & 106.6016 & 35.51624 & 39.48048 & 39.89845 & 12.25704 & 3.341238 & 17.1612 & 2693.75 & 2.293342 & 560.3401 & 0.931211 \\
\hline Anagallis minima & 3.318969 & 7159.185 & 629.2507 & 301.8621 & 108.1912 & 267.7993 & 47.22908 & 53.67138 & 41.58045 & 15.52838 & 4.484567 & 9.976542 & 3019.399 & 0.673771 & 256.2658 & 1.480186 \\
\hline Anagallis tenella & 3.384728 & 5758.012 & 436.6782 & 405.1556 & 95.00838 & 120.6325 & 33.91641 & 52.38126 & 31.68867 & 12.09563 & 3.736515 & 12.00232 & 3171.43 & 0.974765 & 278.3446 & 1.18864 \\
\hline Anchusa arvensis & 4.578853 & 3721.596 & 92.94245 & 371.64 & 112.6595 & 133.1816 & 55.39737 & 57.37906 & 37.13969 & 12.43323 & 2.626966 & 17.92757 & 2442.281 & 2.798679 & 515.7861 & 1.306846 \\
\hline Anchusa officinalis & 6.102222 & 3406.308 & 95.59757 & 386.4145 & 107.4476 & 107.9167 & 45.31918 & 47.96818 & 35.31742 & 12.40084 & 4.652818 & 32.24073 & 2210.329 & 1.571195 & 408.6552 & 1.214869 \\
\hline Andromeda polifolia & 3.710049 & 2653.32 & 20.04692 & 828.2824 & 62.54759 & 173.5735 & 95.93347 & 147.0632 & 35.13238 & 18.77482 & 9.771154 & 5.721451 & 3962.644 & 2.034016 & 360.6986 & 0.458701 \\
\hline Anemone nemorosa & 5.427614 & 5818.883 & 41.24923 & 560.7899 & 66.07784 & 152.2514 & 65.82086 & 75.16424 & 57.23234 & 25.65216 & 7.65494 & 29.98319 & 3897.738 & 2.715884 & 171.6488 & 0.993506 \\
\hline Angelica archangelica & 3.152154 & 6691.121 & 223.4709 & 678.0312 & 128.3957 & 179.4072 & 44.69194 & 58.89403 & 41.38351 & 26.23683 & 5.306177 & 18.26666 & 4814.57 & 0.747151 & 290.3407 & 0.879882 \\
\hline Angelica sylvestris & 5.291821 & 7883.712 & 182.6264 & 534.2982 & 87.57811 & 220.4719 & 56.03874 & 67.21058 & 45.64413 & 24.0398 & 7.478378 & 29.28092 & 4332.42 & 1.481196 & 344.0576 & 0.966121 \\
\hline Anisantha sterilis & 6.16016 & 4172.38 & 71.44652 & 720.3605 & 114.2764 & 129.2138 & 38.20658 & 42.83907 & 31.04505 & 14.88365 & 5.878862 & 27.6088 & 3412.032 & 1.85566 & 541.1221 & 1.056561 \\
\hline Anisantha tectorum & 1.459299 & 3304.303 & 63.56183 & 173.0346 & 91.09811 & 121.0816 & 65.86107 & 56.31977 & 34.19043 & 13.72784 & 2.787053 & 21.11183 & 2028.097 & 1.984129 & 351.6737 & 0.572769 \\
\hline Antennaria dioica & 7.351993 & 2302.798 & 20.57806 & 397.9462 & 52.67561 & 85.46759 & 52.8037 & 62.2562 & 39.11485 & 12.69626 & 5.090902 & 7.794092 & 2303.381 & 0.54036 & 284.2976 & 1.432304 \\
\hline Anthemis arvensis & 3.544868 & 4457.437 & 69.07657 & 474.1378 & 133.7456 & 113.8831 & 31.23274 & 41.71442 & 36.26916 & 11.53527 & 2.418857 & 11.04196 & 2390.133 & 2.701015 & 421.9078 & 0.968335 \\
\hline Anthoxanthum aristatum & 6.261831 & 4016.487 & 73.94596 & 578.6478 & 105.4251 & 112.0004 & 42.18348 & 64.74885 & 48.86008 & 12.52291 & 2.585819 & 13.30047 & 2853.676 & 2.753179 & 478.9505 & 1.221849 \\
\hline Anthoxanthum odoratum & 7.811885 & 9094.018 & 140.7187 & 451.9596 & 127.4241 & 218.9278 & 65.14042 & 85.68595 & 38.49262 & 33.16262 & 7.763766 & 12.71575 & 6976.229 & 2.124331 & 553.9533 & 1.383222 \\
\hline Anthriscus caucalis & 5.43778 & 2559.168 & 61.2544 & 498.9656 & 87.42629 & 98.18323 & 37.85693 & 38.29556 & 33.89342 & 10.81608 & 7.127421 & 27.93848 & 2002.233 & 1.163075 & 383.8071 & 0.700856 \\
\hline
\end{tabular}


Anthriscus sylvestris Anthyllis vulneraria Apera spica-vent Aphanes arvensi

Aphanes inexpectata Apium graveolen

Apium nodiflorun

Arabidopsis thaliana

Arabis glabra

Arabis hirsuta

Arabis hirsuta s. hirsuta

Arctium lappa

Arctium minus (excl. A. pubens)

Arctium p

Arenaria serpyllifolia

Armeria maritim

Arnica montana

Arnoseris minima

Arrhenatherum elatius

henatherum elatius s. elatius

Artemisia campestris s. campestris

Artemisia campestris s. maritima

Artemisia vulgaris

Asparagus officinalis s. officinalı

Asparagus officinalis s. prostratus

Aster tripolium

Athyrium filix-femina

Atriplex littoralis

Atriplex patula

Atriplex prostrat

Avena fatua

Ballota nigra

Ballota nigra s. foetida

Barbarea stricta

Barbarea vulgaris

Berberis vulgaris

Berteroa incana

rula erecta

Betula pendula

Betula pendula + B. pubescens

Betula pubescens

Bidens cernua

Bidens connata

Bidens tripartita

Blackstonia perfoliat

\begin{tabular}{|c|c|c|c|c|c|c|c|c|c|c|c|c|c|c|c|}
\hline .917802 & 5028.874 & 103.2585 & 848.1067 & 97.6112 & 98.86631 & 39.20676 & 46.13021 & 39.17866 & 17.11264 & 6.09453 & 26.15285 & 3201.466 & 1.434572 & 289.5 & 1.31075 \\
\hline 71314 & 3305.387 & 406.7443 & 158.7558 & 82.19233 & 116.5951 & 62.55136 & 52.87829 & 37.54327 & 15.19604 & 3.438752 & 10.70903 & 1903.256 & 0.461212 & 305.4064 & 1.37443 \\
\hline 75989 & 3792.209 & 78.07875 & 42.2507 & 115.6397 & 99.85463 & 30.38285 & 39.95347 & 41.72569 & 10.74224 & 2.880683 & 11.33641 & 2457.404 & 2.642387 & 403.3472 & 0.967747 \\
\hline 22416 & 4018.928 & 73.65564 & 7.1351 & 112.366 & 101.9412 & 30.08751 & 39.55112 & 41.44332 & 11.57936 & 2.952679 & 10.35261 & 2303.67 & 2.654418 & 339.406 & 0.876961 \\
\hline 30071 & 4234.952 & 52.49006 & 1.2151 & 114.0467 & 111.0087 & 45.95206 & 115.0478 & 53.39401 & 13.60524 & 2.62248 & 12.46144 & 2611.098 & 3.326453 & 398.0567 & 1.116251 \\
\hline 00883 & 43.805 & 7.7103 & 17.8038 & 104.5651 & 242.0339 & 36.08179 & 39.94159 & 41.49481 & 16.97301 & 6.008547 & 19.16928 & 6816.045 & 1.438157 & 599.5019 & 0.805333 \\
\hline 629503 & 645.097 & 1.1023 & 7.2368 & 118.5684 & 206.0534 & 53.72538 & 66.47574 & 54.98307 & 27.0229 & 6.257507 & 24.55266 & 6725.265 & 1.301643 & 580.3969 & 0.839749 \\
\hline 768638 & 4633.849 & 63.63401 & 1.5492 & 115.7328 & 135.4405 & 65.08461 & 57.85686 & 39.60557 & 16.36259 & 3.237977 & 13.44873 & 3073.201 & 3.195931 & 508.2633 & 1.199171 \\
\hline 569807 & 2897.392 & 48.09245 & 328.9772 & 99.05438 & 115.792 & 56.87701 & 77.10113 & 41.06219 & 16.40355 & 6.055838 & 24.94991 & 2424.282 & 1.68461 & 392.1011 & 1.226755 \\
\hline 34735 & 279.851 & 38.7585 & 243.0731 & 49.1406 & 59.78147 & 40.78498 & 41.95183 & 35.98285 & 11.33493 & 6.247325 & 13.38103 & 1457.082 & 1.123295 & 225.9012 & 0.718046 \\
\hline 30017 & 952.143 & 42.53969 & 269.943 & 83.6239 & 92.53001 & 57.7858 & 66.58061 & 32.77908 & 12.28486 & 4.539864 & 12.29122 & 1617.088 & 1.785788 & 257.7567 & 0.970443 \\
\hline 86904 & 4431.915 & 105.2655 & 897.0077 & 107.6679 & 128.5858 & 39.63551 & 46.15611 & 41.71276 & 18.95033 & 6.989995 & 23.25756 & 3617.676 & 1.025706 & 401.9987 & 1.335952 \\
\hline 085031 & 4183.32 & .53232 & 9.1573 & 119.5085 & 87.25432 & 38.75205 & 45.19363 & 35.52591 & 12.33407 & 6.04402 & 23.11949 & 3065.553 & 1.078629 & 467.5917 & 1.233932 \\
\hline 432861 & 3565.993 & 131.7203 & 4.9807 & 108.7089 & 101.664 & 38.52304 & 43.70124 & 33.43934 & 12.9707 & 6.987739 & 23.01253 & 3029.523 & 0.887563 & 504.5692 & 1.312827 \\
\hline 938179 & 261.598 & 264.0293 & 199.0447 & 114.214 & 129.922 & 64.69401 & 57.26402 & 29.45815 & 14.42314 & 3.434176 & 11.80148 & 2221.18 & 1.565831 & 363.1853 & 0.710249 \\
\hline 990896 & 954.478 & 583.1083 & 228.3172 & 185.9555 & 249.8925 & 46.40932 & 52.67368 & 31.07785 & 30.2288 & 4.468799 & 13.16717 & 2716.63 & 0.577278 & 385.4498 & 0.887281 \\
\hline 788717 & 726.631 & 14.5475 & 18.6378 & 53.3281 & 100.68 & 59.13965 & 90.34479 & 29.04597 & 16.07503 & 4.314517 & 5.581344 & 3163.29 & 0.651358 & 259.4707 & 0.602473 \\
\hline 965136 & 3545.042 & 72.7796 & 518.487 & 89.73277 & 92.18603 & 30.52101 & 43.43239 & 44.55757 & 9.609985 & 2.77194 & 8.368597 & 491.03 & 2.397483 & 284.031 & 0.672996 \\
\hline 168792 & 5300.969 & 67.36348 & 6.1643 & 98.92802 & 100.0029 & 37.22202 & 45.70093 & 48.61617 & 16.02817 & 5.154788 & 17.67635 & 3363.752 & 1.349317 & 334.0468 & 1.206413 \\
\hline 662947 & 77.523 & 76.05932 & 573.8088 & 91.80158 & 88.9086 & 28.78676 & 34.59832 & 45.32042 & 12.75918 & 4.85749 & 18.70472 & 949.827 & 1.428609 & 00.4877 & 1.781071 \\
\hline 677949 & 2553.406 & 29.14943 & 130.193 & 64.87223 & 73.72953 & 31.59768 & 39.49602 & 22.8903 & 8.907786 & 3.868582 & 12.26081 & 435.53 & 1.274897 & 17.6502 & 1.176951 \\
\hline . 083691 & 91.326 & 797.8153 & 128.0212 & 1.18491 & 101.0923 & 68.30115 & 55.66708 & 28.9741 & 15.56056 & 2.898366 & 11.09469 & 860.84 & 2.065603 & 5.8609 & 0.633219 \\
\hline .963758 & 3770.168 & 171.8257 & 616.0678 & 113.6484 & 113.5137 & 37.38379 & 45.41746 & 46.23368 & 14.67581 & 6.109192 & 23.47951 & 3188.334 & 1.754297 & 440.1564 & 1.222802 \\
\hline 1.503359 & 2270.191 & 31.12444 & 469.6786 & 45.39761 & 55.90643 & 31.20988 & 23.65408 & 23.68389 & 9.337674 & 4.040109 & 14.69685 & 1212.403 & 0.827446 & 84.6438 & 0.77628 \\
\hline 5.75766 & 2771.057 & .46205 & 389.8216 & 81.34731 & 95.33047 & 44.17633 & 46.76849 & 36.15005 & 12.86482 & 6.158631 & 23.02838 & 1858.24 & 1.27542 & 2.5668 & .138549 \\
\hline . 272641 & 2749.239 & 37.17723 & 167.0979 & 67.82952 & 104.694 & 51.54708 & 43.11077 & 29.04778 & 14.42242 & 4.482425 & 10.02432 & 816.37 & 1.773498 & 88.9447 & 728597 \\
\hline 91751 & 6989.41 & 590.4743 & 690.559 & 112.5547 & 228.7521 & 28.91261 & 30.53727 & 45.09732 & 20.57311 & 6.642938 & 22.23854 & 3785.729 & 1.647504 & 367.8843 & 0.822976 \\
\hline 735604 & 3711.889 & 59.26832 & 323.3541 & 70.13533 & 147.339 & 47.90556 & 60.45724 & 40.15899 & 18.10657 & 7.709 & 21.07448 & 2884.47 & 1.51674 & 233.7084 & 1.01988 \\
\hline 1.959782 & 7775.087 & 671.7371 & 487.4805 & 152.6172 & 290.7664 & 30.99729 & 35.85348 & 39.0149 & 21.10922 & 4.248836 & 27.77593 & 3153.79 & 1.650032 & 526.4811 & 0.876241 \\
\hline .024056 & 4477.717 & 580.0375 & 617.6802 & 132.666 & 119.5581 & 37.33779 & 47.51263 & 52.99623 & 18.21138 & 5.007755 & 16.61366 & 4292.317 & 1.598998 & 3.6766 & 1.296611 \\
\hline 4.37594 & 6720.447 & 691.7886 & 848.0858 & 134.8614 & 179.3497 & 40.64212 & 48.46856 & 58.63921 & 27.89041 & 7.46417 & 24.77281 & 5598.246 & 1.53275 & 622.8964 & 0.997809 \\
\hline 4.459046 & 3440.392 & 298.1313 & 767.1499 & 126.1406 & 96.88726 & 30.34212 & 37.80876 & 49.63031 & 10.34865 & 3.047817 & 13.1871 & 2542.409 & 2.889716 & 307.3122 & 1.115347 \\
\hline 011378 & 2445 & 62.6148 & 781.2641 & 74.75321 & 72.69144 & 26.39806 & 30.36376 & 20.92568 & 9.021173 & 4.564978 & 24.10186 & 2550.037 & 1.127905 & 544.5954 & 1.192784 \\
\hline 942911 & 3125.68 & 0.7724 & 631.9996 & 126.9707 & 76.98683 & 35.17886 & 39.53655 & 25.55281 & 11.63045 & 6.720943 & 27.3332 & 2308.65 & 1.003517 & 31.5308 & 1.337266 \\
\hline 076517 & 7766.225 & 132.7145 & 2.478 & 128.089 & 298.8809 & 69.39081 & 92.20175 & 65.67731 & 35.58728 & 11.57268 & 24.86925 & 9162.625 & 1.604271 & 626.6085 & 0.943078 \\
\hline 42144 & 7398.622 & 9.0989 & 6.0582 & 109.9605 & 172.7329 & 51.97546 & 65.9588 & 66.38892 & 36.25841 & 10.13587 & 31.40094 & 17.428 & 2.06861 & 44.315 & 1.058326 \\
\hline 71725 & 2397.876 & 153 & .0833 & 998 & 82.89375 & 33 & 123 & 31.10542 & 11.68204 & 5.432372 & 28.92366 & 091 & 1.991344 & 06.4007 & 1.952016 \\
\hline .024229 & 7050.361 & 18574 & 44 & 82 & 112.1241 & 39.98373 & 787 & 87 & 46 & 3.00154 & 229 & 676 & 2.137313 & 272 & 1.324257 \\
\hline .921223 & 4955.962 & 51 & 512.7483 & 78428 & 7.6363 & & 3026 & 3052 & 22.32929 & 9.755419 & 27.07465 & 7.936 & 1.085107 & 99.4375 & 0.891057 \\
\hline .065446 & 2549.655 & .08344 & 4356 & 00721 & 73 & 64.49637 & .83722 & 49.75176 & 5.65661 & 10.69583 & 25.08363 & 0.869 & 2.493587 & 8334 & 1.382535 \\
\hline 469253 & 16.497 & .3419 & 7033 & 18442 & 1.4198 & 72.56579 & 89.74038 & 70.02363 & 1205 & 7.431521 & 26.50254 & 4.884 & 2.785364 & 348.0981 & 2.02266 \\
\hline .123633 & 4.391 & 44584 & 9323 & 78943 & 0.469 & 98263 & 24.5713 & 59.43152 & 23.77947 & 10.12633 & 29.05384 & 31.372 & 2.504123 & 65.232 & 1.339356 \\
\hline .909961 & 6.934 & 239.9132 & 4067 & 97.69237 & 9.3864 & 27.83775 & 38.11561 & 44312 & 23.59808 & 73 & 27.92598 & 11 & 1.244754 & 21.2032 & 1.413503 \\
\hline 903588 & 9.764 & 247.699 & 1918 & 19 & 0.2026 & 37.46872 & 433 & 39.71986 & 24.49019 & 7.999397 & 28.88317 & 23.565 & 04629 & 0.0761 & 1.101391 \\
\hline 93432 & .554 & 548 & & 3958 & 3523 & 33 & 8836 & 52.35249 & 14 & & 574 & 516 & 0725 & 2361 & 1.48905 \\
\hline 70902 & & 22 & 105 & 01 & 32 & 444 & & 194 & & & 334 & 991 & & 87 & \\
\hline
\end{tabular}


Blechnum spicant

Blysmus compressus

Bolboschoenus maritim

Botrychium lunaria

Brachypodium pinnatum

Brachypodium sylvaticum

Brassica napus

Brassica nigra

Briza media

Bromopsis erecta

Bromopsis inermis

Bromus hordeaceus

Bromus hordeaceus s. hordeaceus

Bromus hordeaceus s. thominei

Bromus racemosus

Bromus racemosus s. racemosu

Bryonia dioica

Butomus umbellatus

Calamagrostis canescens

Calamagrostis epigejos

Calamagrostis stricta

Calammophila baltic

Calla palustri

Callitriche obtusangula

Callitriche platycarpa

Callitriche stagnal

Caltha palustris

Caltha palustris s. araneosa

Caltha palustris s. palustris

Calystegia sepium

Campanula rapunculoide

Campanula rapunculus

Campanula rotundifolia

Campanula trachelium

Capsella bursa-pastoris

Cardamine amar

Cardamine hirsuta

Cardamine pratensis

uus crispus

Carex acuta

Carex acutiformis

Carex appropinquata

Carex aquatilis

Carex arenaria

Carex buxbaumii

Carex caryophyllea

\begin{tabular}{|c|c|c|c|c|c|c|c|c|c|c|c|c|c|c|c|}
\hline .923841 & 2434.219 & 39.27601 & 599.7043 & 54.18333 & 79.20842 & 55.93702 & 63.27959 & 45.74744 & 11.77391 & 9.076017 & 20.29973 & 1996.581 & 2.37855 & 399.036 & 419 \\
\hline 205941 & 4863.91 & 550.196 & 377.3149 & 102.4592 & 130.2754 & 28.24198 & 24.1018 & 33.40647 & 9.858232 & 3.797471 & 7.463809 & 3171.057 & 0.683848 & 259.2465 & $1.02 \mathrm{c}$ \\
\hline 632602 & 6473.107 & 612.7749 & 558.4623 & 87.17716 & 185.5524 & 43.02972 & 50.70151 & 43.66441 & 28.33355 & 7.284235 & 26.55229 & 7998.864 & 1.183469 & 667.6459 & 1.091242 \\
\hline 454502 & 2876.083 & .42556 & 4.7209 & 60035 & 89.29671 & 56.32005 & 52.94716 & 38.76952 & 4.98199 & 4.976253 & 11.84044 & 1700.804 & 0.83246 & 222.6605 & 1.841325 \\
\hline 473399 & 278.536 & 29.90269 & 4.8972 & 0.26512 & 3.00102 & 41.02539 & 7.10311 & 45.01094 & .976089 & 3.65655 & 18.11525 & 2070.787 & 0.496397 & 286.2895 & 0.523899 \\
\hline 490714 & 2716.586 & 37.60204 & 8.9325 & 47.17941 & 8.8878 & 35.37836 & 28.60818 & 30.2557 & 9.196525 & 5.24672 & 16.799 & 1502.824 & 1.679856 & 160.2837 & 1.41841 \\
\hline 5.20698 & 5049.446 & 93.04177 & 9.7947 & 110.4131 & 125.8455 & 38.17727 & 48.75633 & 50.47833 & 32.82042 & 5.987314 & 24.65295 & 5500.824 & 2.880033 & 339.5658 & 1.203559 \\
\hline 4.89283 & 3742.623 & 183.4604 & 741.0835 & 107.3435 & 99.14015 & 40.64295 & 50.21082 & 56.09445 & 19.93123 & 6.599761 & 25.24037 & 4416.675 & 1.288171 & 415.3854 & 0.812194 \\
\hline 350899 & 5688.486 & 60.86742 & 342.684 & 75.57408 & 166.9478 & 60.97069 & 65.45428 & 35.65588 & 31.40005 & 5.099984 & 10.30544 & 6774.578 & 0.591834 & 283.6968 & 1.367952 \\
\hline 410618 & 4146.873 & 63.64121 & 391.2757 & 135.815 & 93.26099 & 72.59168 & 72.8692 & 36.85638 & 10.5053 & 4.037082 & 9.232763 & 3954.857 & 0.497815 & 318.852 & 0.625493 \\
\hline 16509 & 3940.415 & 100.3757 & 426.4089 & 121.3292 & 78.9854 & 33.66868 & 37.60089 & 42.57812 & 14.09942 & 4.033825 & 17.99382 & 3167.416 & 1.261999 & 317.8794 & 0.47328 \\
\hline 67526 & 9154.073 & 469.5632 & 429.1378 & 190.7224 & 155.8583 & 62.73286 & 67.58658 & 40.23734 & 24.3104 & 4.933195 & 10.99491 & 5709.896 & 1.152688 & 617.3461 & 0.985351 \\
\hline 94977 & 7276.572 & 117.115 & 444.124 & 173.1357 & 171.0562 & 53.78975 & 60.47721 & 30.43173 & 21.37061 & 5.148915 & 14.72908 & 5218.492 & 1.184691 & 582.0282 & 0.945672 \\
\hline 231408 & 2230.077 & 31.75428 & 90.069 & 2.98225 & 88.73728 & 62.64702 & 48.92474 & 36.6589 & 10.15922 & 3.268834 & 7.513131 & 1621.091 & 1.582056 & 72.3333 & 1.168869 \\
\hline 313274 & 5102.77 & 118.2213 & 410.7751 & 97.38088 & 112.9403 & 29.01913 & 31.72466 & 47.5103 & 21.13727 & 4.434541 & 24.04781 & 5140.793 & 0.483003 & 477.0409 & 0.508009 \\
\hline 083285 & 5397.525 & 503.8881 & 9.0727 & 5.18027 & 91.9497 & 26.63362 & 31.74859 & 25.04795 & 23.62682 & 2.87213 & 7.379419 & 5186.285 & 0.853192 & 409.4413 & 0.627152 \\
\hline 5.19833 & 2712.927 & .93622 & 2.7862 & 3.24686 & 91.14265 & 35.06221 & 39.6548 & 30.51484 & 12.54781 & 5.775227 & 24.98696 & 2562.386 & 0.894496 & 07.5128 & 1.364293 \\
\hline 1.918605 & 4804.055 & 9.1297 & 326.9871 & 71.17932 & 171.5044 & 19.83956 & 24.68806 & 25.15016 & 18.61872 & 6.040817 & 25.2735 & 4750.427 & 0.998956 & 435.3677 & 0.60382 \\
\hline 843936 & 391.084 & 8.3347 & 5.8608 & 111.408 & 297.7504 & 72.12063 & 100.1605 & 48.40465 & 31.37378 & 9.384631 & 28.61297 & 5115.902 & 1.94619 & 15.7781 & 1.032767 \\
\hline 36517 & 60.611 & 0.2066 & 0.5919 & 114.4959 & 228.1687 & 92.62593 & 92.95845 & 72.66028 & 19.45103 & 7.299816 & 25.30653 & 2973.28 & 1.651675 & 86.0901 & 1.920254 \\
\hline 083923 & 4876.757 & .92398 & 10.9347 & 126.1009 & 154.4432 & 21.61581 & 33.14666 & 16.25796 & 16.92587 & 775405 & 11.08027 & 3007.911 & 1.165774 & 3.3049 & 0.499746 \\
\hline 782324 & 2502.416 & 6.9534 & 3.4964 & 82.85709 & 113.4177 & 59.83348 & 54.59245 & 80.7648 & 10.59987 & 060972 & 24.43252 & 554.288 & 1.575681 & 22.4945 & 0.693976 \\
\hline 140797 & 7833.398 & 253.0464 & 492.7202 & 137.3333 & 191.7684 & 45.73958 & 66.88717 & 36.41889 & 25.42359 & 9.877346 & 21.94586 & 85.798 & 1.755064 & 883.595 & 1.659142 \\
\hline .576429 & 5871.248 & 276.2012 & 53.2639 & 98.50083 & 198.6017 & 30.16812 & 41.37767 & 35.33381 & 17.46617 & .87428 & 18.4027 & 10.013 & 1.140911 & 499.4515 & 1.791732 \\
\hline 987208 & 7897.308 & 3.5109 & 5.1569 & 135.7921 & 220.746 & 39.38986 & 50.90038 & 40.56305 & 20.53979 & 073934 & 24.04247 & 52.622 & 1.602145 & 9.2381 & 189619 \\
\hline .740211 & 8267.314 & 365.7433 & 717.8272 & 130.8608 & 251.7528 & 44.79158 & 60.59897 & 44.73139 & 24.68669 & 7.425876 & 24.59848 & 6490.237 & 1.526667 & 489.583 & .610045 \\
\hline 64584 & 5305.25 & 2.1184 & 507.9216 & 110.2764 & 212.3269 & 42.08521 & 56.72667 & 33.86994 & 19.7542 & 8.935885 & 29.88282 & 4912.742 & 1.207913 & 5.6212 & 0.683091 \\
\hline 605539 & 7573.415 & 235.2308 & 651.223 & 103.101 & 152.6846 & 49.45267 & 63.16196 & 40.29945 & 23.09523 & 5.473145 & 18.64822 & 5335.368 & 1.633878 & 256.2586 & 0.445689 \\
\hline 549273 & 7231.082 & 176.6682 & 508.6812 & 123.0899 & 218.6025 & 51.75563 & 71.92024 & 38.1028 & 24.77984 & 8.171783 & 26.57618 & 6325.213 & 1.08275 & 631.7317 & 0.866275 \\
\hline 003482 & 7470.553 & 280.7587 & 688.1358 & 110.2391 & 228.2315 & 57.61742 & 68.53785 & 71.24046 & 28.78283 & 10.52874 & 23.07547 & 5234.97 & 1.619543 & 21.3833 & 1.511862 \\
\hline .710623 & 3728.263 & 109.4846 & 419.2942 & 145.2857 & 80.25973 & 50.40635 & 48.25568 & 40.4761 & 13.0507 & 4.556555 & 21.59207 & 2254.26 & 2.001237 & 381.997 & 1.20782 \\
\hline 847894 & 3663.252 & 49.64211 & 323.5687 & 70.09315 & 76.71916 & 29.02731 & 34.47939 & 26.24204 & 10.89187 & 3.628154 & 10.71953 & 2425.833 & 0.89582 & 237.7801 & 0.675705 \\
\hline 580132 & 4999.996 & 33.40872 & 381.4606 & 105.1778 & 127.0592 & 55.12649 & 81.098 & 32.67798 & 15.54051 & 7.544083 & 14.09641 & 2695.556 & 0.825217 & 322.2633 & 2.27733 \\
\hline 13251 & 2455.716 & 30.43923 & 477.7585 & 40.97868 & 52.67182 & 32.87018 & 25.03339 & 25.10354 & 9.518891 & 4.733105 & 16.91209 & 1152.286 & 1.740656 & 151.1625 & 0.910008 \\
\hline 187113 & 27.097 & 1.9725 & 563.138 & 49.6444 & 124.1287 & 31.5471 & 38.97353 & 37.94702 & 16.1972 & 3.998473 & 12.51046 & 4051.549 & 2.19281 & 526.1039 & 0.738468 \\
\hline 816814 & 668.009 & 5.0512 & .3717 & 2617 & 220.3831 & 62.48199 & 30.6525 & 58.77455 & 25.99715 & 7.319522 & 20.9311 & 97.894 & 1.181649 & 409.4445 & 1.218722 \\
\hline .792463 & 341.903 & 92.68561 & .2958 & 126.8957 & 160.6813 & 29097 & 75.02358 & 42.31238 & 18.93886 & 6.529522 & 21.30192 & 90.892 & 1.11543 & 405.633 & 1.183629 \\
\hline 6.3533 & 4.129 & 1.005 & 1.0025 & & 25 & 40481 & 603 & 304 & 23.11326 & 11 & 207 & 11 & 1.190164 & 717.0877 & 0.91007 \\
\hline 587141 & 1.137 & 5.4576 & 9897 & 37 & 6.9544 & 55603 & 026 & 47.49483 & 1127 & 6.909193 & 25.89951 & 348.403 & 1.297168 & 82.464 & 0.968132 \\
\hline .074931 & 70.24 & 8.5009 & .2288 & .8263 & 4.9067 & 43897 & 5.99546 & 35.91655 & 21.38531 & 267 & 25.64794 & 88.701 & 1.202713 & 09.843 & 1.048271 \\
\hline 020723 & 7898.408 & 185.6346 & 1.9774 & 8906 & 2.6578 & 0874 & 79.26966 & 37.42153 & 27.26847 & 10.46444 & 28.07608 & 5097.422 & 1.778053 & .2346 & 1.033827 \\
\hline 986419 & 5024.726 & 115.9006 & . 0969 & 66898 & 6.6728 & 83961 & 1034 & 30.32943 & 20.96445 & 11.11767 & 32.35612 & 3230.37 & 2.350136 & 7.9094 & 0.620581 \\
\hline .558601 & 3813.731 & 155.37 & 2.7838 & 22611 & .3375 & 26.07671 & 08058 & 26.65118 & 17.07512 & 761 & 18.61974 & 4644.849 & 1.422811 & 8.5022 & 0.458891 \\
\hline 37242 & 8.143 & 445.4461 & 39 & 268 & .4607 & 22408 & . 49167 & & 189 & 09258 & 14.41506 & 8.682 & 2.159434 & 61.2185 & 2.118836 \\
\hline 870509 & 829 & & & & & 77 & 46 & & 008 & & 343 & .358 & 576 & 673 & J11 \\
\hline 3225 & .965 & 192 & 7254 & 94 & 5.8527 & 1) & & & 51 & & & 31 & & 77 & \\
\hline
\end{tabular}




\begin{tabular}{|c|c|c|c|c|c|c|c|c|c|c|c|c|c|c|c|c|}
\hline Carex curta & 7.087039 & 9017.913 & 135.805 & 492.8953 & 120.5868 & 291.174 & 66.15641 & 94.70051 & 37.83099 & 30.91359 & 9.233554 & 25.67895 & 3819.779 & 2.565237 & 756.4937 & 0.93208 \\
\hline Carex diandra & 3.191837 & 5097.72 & 106.1225 & 387.8307 & 113.1938 & 154.5049 & 24.01242 & 34.86272 & 19.74984 & 17.7163 & 7.936573 & 16.51324 & 2640.03 & 1.351722 & 640.4185 & 0.558973 \\
\hline Carex distans & 3.643217 & 6623.47 & 583.159 & 370.068 & 117.4977 & 180.0585 & 32.70573 & 36.04531 & 33.87683 & 18.55119 & 5.245054 & 12.68865 & 4325.183 & 0.903146 & 264.7741 & 0.741257 \\
\hline Carex disticha & 3.481877 & 4955.343 & 278.3809 & 344.0564 & 111.6339 & 189.7406 & 33.16759 & 38.69893 & 32.11212 & 22.27367 & 5.762907 & 13.76287 & 4643.023 & 0.77205 & 678.7461 & 0.705843 \\
\hline Carex echinata & 7.750849 & 7778.611 & 91.58281 & 499.4647 & 81.00306 & 253.3973 & 47.65797 & 67.62564 & 31.7836 & 24.64159 & 9.664886 & 12.39303 & 3827.117 & 1.959157 & 527.7781 & 0.877483 \\
\hline Carex elata & 5.466905 & 8552.593 & 119.1384 & 483.8257 & 118.1626 & 256.0602 & 63.83238 & 91.39267 & 45.00238 & 28.31232 & 9.322394 & 33.40266 & 4744.234 & 1.759795 & 711.2355 & 0.867784 \\
\hline Carex elongata & 4.321779 & 4976.554 & 67.82459 & 282.1901 & 82.49636 & 224.3331 & 59.66011 & 83.80595 & 38.32211 & 20.69244 & 7.020086 & 19.57003 & 3312.871 & 1.19721 & 307.2107 & 0.865294 \\
\hline Carex flacca & 6.12937 & 6935.277 & 608.9873 & 416.5612 & 108.935 & 253.7179 & 77.47113 & 73.7438 & 56.12007 & 22.07382 & 4.281322 & 13.49422 & 4458.172 & 0.714423 & 349.4071 & 1.459334 \\
\hline Carex hirta & 5.04579 & 8379.65 & 256.6891 & 520.2966 & 148.3562 & 152.6568 & 50.73977 & 62.58004 & 38.54369 & 22.81458 & 5.490915 & 15.33433 & 5741.728 & 0.948031 & 551.9252 & 1.038685 \\
\hline Carex hostiana & 2.922094 & 3522.914 & 68.29254 & 327.6882 & 55.46135 & 146.8369 & 27.21069 & 31.41843 & 16.01679 & 15.46662 & 3.575798 & 6.855322 & 3193.84 & 0.690723 & 227.9598 & 0.543028 \\
\hline Carex lasiocarpa & 4.995334 & 7293.938 & 122.4892 & 393.7773 & 124.6152 & 167.8981 & 29.50787 & 41.61765 & 26.83114 & 22.01031 & 9.20421 & 20.6445 & 3566.592 & 2.350581 & 650.4065 & 0.751137 \\
\hline Carex ligerica & 2.282975 & 3536.528 & 28.85434 & 165.9289 & 96.55149 & 103.6472 & 53.59839 & 73.33449 & 41.04752 & 13.57247 & 3.636829 & 8.750591 & 1843.878 & 0.898738 & 269.6761 & 2.072693 \\
\hline Carex muricata & 10.14791 & 8199.278 & 440.0956 & 733.5952 & 156.0564 & 243.0645 & 90.77234 & 103.2591 & 63.66072 & 28.25543 & 10.96513 & 32.06421 & 6882.402 & 1.111036 & 717.1701 & 2.341221 \\
\hline Carex nigra & 9.416023 & 8990.742 & 457.4416 & 480.1341 & 126.2542 & 267.7431 & 57.59287 & 82.57872 & 39.79164 & 28.66569 & 9.490563 & 14.33372 & 5096.076 & 1.781275 & 718.0105 & 1.254815 \\
\hline Carex nigra $x$ trinervis & 4.721666 & 7111.363 & 344.8337 & 242.328 & 118.1802 & 144.0374 & 19.58498 & 41.29952 & 20.00677 & 11.42653 & 6.207126 & 12.51878 & 3262.835 & 2.039451 & 509.7638 & 0.99885 \\
\hline Carex oederi & 6.424305 & 7239.697 & 716.246 & 573.0299 & 127.0054 & 218.4507 & 40.75032 & 64.02845 & 38.42021 & 19.43286 & 5.570114 & 17.46589 & 6333.581 & 0.730531 & 368.2424 & 2.047964 \\
\hline Carex oederi s. oederi & 5.197935 & 4904.649 & 544.9691 & 413.74 & 83.24394 & 179.9564 & 36.34122 & 61.05129 & 33.44533 & 18.68666 & 5.033141 & 12.98126 & 4676.265 & 0.915991 & 281.8876 & 1.627616 \\
\hline Carex oederi s. oedocarpa & 4.851311 & 6167.108 & 465.7201 & 461.7863 & 80.29416 & 173.985 & 40.77432 & 50.63073 & 34.98388 & 19.09568 & 5.318062 & 10.77953 & 4297.978 & 0.924827 & 360.4192 & 0.877099 \\
\hline Carex otrubae & 3.829653 & 6261.648 & 526.5384 & 482.2797 & 94.86842 & 151.6796 & 34.16074 & 43.60076 & 36.15446 & 27.41765 & 5.58985 & 20.59021 & 7837.627 & 0.969492 & 517.6434 & 0.9074 \\
\hline Carex ovalis & 5.723254 & 9008.044 & 106.4281 & 430.4192 & 120.9445 & 178.1192 & 54.80265 & 73.77177 & 30.96131 & 25.1004 & 4.76792 & 11.77216 & 4705.638 & 1.206465 & 392.127 & 0.978537 \\
\hline Carex pallescens & 6.311 & 6841.782 & 60.48709 & 624.3282 & 73.44438 & 173.9569 & 62.32006 & 76.29957 & 42.44315 & 23.62583 & 7.640376 & 25.69468 & 3872.293 & 2.003093 & 243.6196 & 1.010693 \\
\hline Carex panicea & 8.108389 & 7549.973 & 344.6368 & 445.9767 & 94.73905 & 238.5175 & 52.37134 & 68.29779 & 31.78254 & 25.19345 & 5.618205 & 10.77723 & 5350.274 & 1.192274 & 402.897 & 1.144678 \\
\hline Carex paniculata & 5.18494 & 7909.883 & 186.7272 & 433.1012 & 125.2603 & 224.8247 & 57.9494 & 78.09365 & 35.70489 & 25.29508 & 8.926885 & 33.83385 & 3901.478 & 2.1452 & 706.1344 & 1.017739 \\
\hline Carex pilulifera & 7.877735 & 1931.163 & 28.77304 & 837.8209 & 53.48562 & 80.83038 & 60.79089 & 77.24736 & 52.2483 & 13.81431 & 10.87532 & 10.27736 & 2456.35 & 3.174322 & 378.6168 & 1.176255 \\
\hline Carex pseudocyperus & 4.922115 & 7991.315 & 186.9272 & 399.5067 & 131.5735 & 231.5299 & 58.00921 & 82.82989 & 44.29674 & 23.81052 & 9.400132 & 23.3884 & 5310.86 & 1.215965 & 689.7342 & 1.027112 \\
\hline Carex pulicaris & 3.324296 & 4482.895 & 82.15184 & 415.8314 & 57.49001 & 149.368 & 30.64148 & 40.26314 & 18.31633 & 20.01557 & 3.646719 & 7.717575 & 3772.315 & 0.677776 & 250.0673 & 0.650371 \\
\hline Carex remota & 4.337793 & 3998.656 & 73.36386 & 324.6912 & 74.5053 & 157.7168 & 48.83383 & 60.93435 & 44.9776 & 17.07598 & 7.809746 & 17.90395 & 3214.381 & 1.374529 & 278.387 & 0.945763 \\
\hline Carex riparia & 5.499837 & 7916.688 & 274.8734 & 570.9794 & 132.5646 & 237.261 & 58.67966 & 78.45078 & 43.08329 & 26.48021 & 10.60256 & 28.32818 & 5535.831 & 1.566954 & 668.8415 & 1.109767 \\
\hline Carex rostrata & 8.044143 & 8278.833 & 171.4568 & 416.4233 & 140.7363 & 189.8903 & 33.59022 & 44.15257 & 31.04013 & 26.48925 & 9.11318 & 22.30676 & 4803.099 & 2.104989 & 500.6664 & 1.037806 \\
\hline Carex spicata & 5.614842 & 7056.141 & 127.2316 & 435.807 & 114.9058 & 132.442 & 43.02478 & 53.88074 & 43.12382 & 21.06664 & 4.900689 & 14.57267 & 4906.24 & 0.818818 & 366.7836 & 0.989264 \\
\hline Carex sylvatica & 3.729006 & 2758.827 & 34.20535 & 379.1102 & 47.84919 & 64.87154 & 34.10336 & 28.29636 & 24.42032 & 9.817749 & 5.044787 & 14.03296 & 1391.862 & 1.603801 & 145.5415 & 1.26337 \\
\hline Carex trinervis & 6.679536 & 9376.831 & 505.8098 & 401.8395 & 139.477 & 347.7506 & 65.60058 & 120.0102 & 38.00583 & 27.46388 & 4.730035 & 13.25277 & 5288.716 & 1.218276 & 360.458 & 1.345686 \\
\hline Carex vesicaria & 5.563237 & 7991.199 & 132.4397 & 462.7213 & 134.8492 & 241.4052 & 52.21279 & 74.98577 & 45.42762 & 25.68618 & 9.350958 & 23.6048 & 4756.062 & 1.781107 & 522.3128 & 0.964462 \\
\hline Carlina vulgaris & 3.570561 & 3127.752 & 45.75152 & 281.3109 & 74.3358 & 94.3145 & 46.07501 & 46.88414 & 30.05321 & 14.00804 & 5.141064 & 11.72391 & 1856.498 & 0.563862 & 259.898 & 0.938919 \\
\hline Carpinus betulus & 4.649172 & 2892.222 & 33.94636 & 496.0545 & 57.63523 & 86.8977 & 33.27853 & 41.49633 & 28.34147 & 10.63092 & 9.347994 & 21.59383 & 1266.323 & 3.232308 & 177.6958 & 1.14993 \\
\hline Carum carvi & 1.476317 & 3609.996 & 54.74714 & 297.7992 & 71.51862 & 61.64912 & 24.67779 & 27.13452 & 19.57367 & 17.4601 & 2.167891 & 5.752199 & 2701.791 & 0.666868 & 315.4788 & 0.451318 \\
\hline Centaurea cyanus & 3.643836 & 3727.012 & 74.8549 & 534.7347 & 112.6923 & 100.1915 & 27.6397 & 37.52988 & 41.76744 & 10.04198 & 2.91443 & 11.01705 & 2366.716 & 2.581832 & 311.5723 & 1.034307 \\
\hline Centaurea jacea & 4.474746 & 6078.097 & 73.61537 & 409.0391 & 92.1015 & 136.5764 & 49.91862 & 54.13378 & 34.48227 & 25.37866 & 4.933018 & 12.52852 & 5706.889 & 0.785058 & 346.6477 & 1.277664 \\
\hline Centaurea scabiosa & 1.656692 & 3005.789 & 43.75445 & 501.9518 & 69.00757 & 80.3512 & 34.97955 & 32.96205 & 32.46912 & 8.824057 & 3.785593 & 10.75127 & 2026.66 & 0.532784 & 299.478 & 0.382322 \\
\hline Centaurium erythraea & 4.989057 & 5167.421 & 792.5633 & 446.3762 & 107.1705 & 226.0895 & 52.5317 & 56.17221 & 41.68402 & 20.96947 & 5.377852 & 16.40783 & 3796.396 & 0.746114 & 362.3947 & 1.528362 \\
\hline Centaurium littorale & 3.923851 & 7431.806 & 603.3216 & 313.7809 & 121.8813 & 272.0877 & 56.48047 & 58.58968 & 48.60915 & 22.58451 & 6.892748 & 13.69323 & 3495.416 & 0.817875 & 336.7772 & 1.0252 \\
\hline Centaurium pulchellum & 2.992742 & 7827.74 & 608.5898 & 398.2535 & 148.4283 & 275.3843 & 46.25251 & 44.78941 & 47.07344 & 21.92633 & 5.928306 & 15.96163 & 2842.698 & 1.215135 & 275.0752 & 0.770148 \\
\hline Cerastium arvense & 3.365995 & 4149.895 & 52.85565 & 247.2745 & 110.8088 & 103.0863 & 54.52676 & 57.20718 & 29.71306 & 15.81751 & 4.621263 & 12.24504 & 2294.58 & 1.45093 & 325.64 & 1.007643 \\
\hline Cerastium diffusum & 1.496965 & 3053.723 & 753.7391 & 127.1886 & 80.89437 & 133.0929 & 80.40401 & 83.38015 & 71.13488 & 14.57267 & 1.952594 & 12.85854 & 1679.997 & 0.374326 & 275.8483 & 0.831516 \\
\hline Cerastium fontanum s. holosteoides & 4.837127 & 6773.504 & 135.195 & 376.5275 & 141.2358 & 149.5916 & 26.55595 & 33.70897 & 19.62912 & 19.26117 & 3.125361 & 11.54135 & 4824.441 & 0.538365 & 580.6016 & 0.74114 \\
\hline Cerastium fontanum s. vulgare & 4.264609 & 7905.118 & 360.9481 & 468.8564 & 139.469 & 162.3429 & 49.0031 & 59.95013 & 30.5059 & 26.56213 & 5.299997 & 13.90107 & 37.852 & 1.113131 & 588.1749 & 1.16626 \\
\hline
\end{tabular}


Cerastium glomeratum

Cerastium semidecandrum

Ceratocapnos claviculat

Ceratophyllum demersum

Chaerophyllum temulum

Chamerion angustifoliu

Chelidonium majus

Chenopodium album

Chenopodium ficifolium

Chenopodium glaucum

Chenopodium polyspermum

nopodium rubrum

Chrysanthemum segetum

Chrysosplenium alternifolium

Chrysosplenium oppositifolium

Cichorium intybus

Cicuta virosa

Circaea lutetiana

Cirsium acaule

Cirsium dissectum

Cirsium dissectum x palustre

Cirsium oleraceum

Cirsium palustre

Cladium mariscus

Claytonia perfoliata

Clematis vitalba

Clinopodium acinos

Clinopodium vulgare

Cochlearia danica

Cochlearia officinalis s. officinalis

Colchicum autumnale

Convallaria maja

Convolvulus arvensis

Corispermum intermediun

Cornus sanguinea

Coronopus squamatus

Corydalis solida

Corylus avellana

Corynephorus canescens

Crataegus laevigata

Crataegus monogyna

Crepis biennis

Crepis capillaris

Crepis paludosa

Crepis tectorum

\begin{tabular}{|c|c|c|c|c|c|c|c|c|c|c|c|c|c|c|c|}
\hline 4.86028 & 8745.943 & 112.1629 & 516.068 & 174.8063 & 182.0143 & 52.56348 & 66.70668 & 44.03393 & 32.12004 & 5.028834 & 17.22445 & 6874.508 & 2.282964 & 795.6515 & . 079 \\
\hline 2.44445 & 739.839 & 58.89439 & 168.1777 & 59.58492 & 97.77941 & 62.62915 & 54.04669 & 28.33869 & 12.30719 & 3.679809 & 11.14357 & 1722.901 & 1.620734 & 286.3297 & 1.305502 \\
\hline 223567 & 2539.54 & 37.23294 & 501.9613 & 63.78915 & 94.19926 & 57.32716 & 76.71878 & 37.56909 & 16.45612 & 9.632552 & 24.02457 & 2344.171 & 2.31345 & 488.119 & 1.293081 \\
\hline 12445 & 083.168 & 209.5222 & 7.4196 & 71.1933 & 184.1227 & 19.17827 & 5.80051 & 23.59215 & 18.09788 & 6.16387 & 25.74276 & 4525.998 & 1.204773 & 427.1799 & 0.709884 \\
\hline 37861 & 821.164 & 3.9536 & 9.7889 & 131.8053 & 10.6507 & 25.53447 & .53154 & 33.76043 & 11.39702 & 3.224757 & 14.70212 & 2508.489 & 1.286957 & 409.8825 & 0.56296 \\
\hline 26994 & 19.677 & 38.35982 & 9.1554 & 66.50782 & 2.3412 & 32.79725 & 32.01655 & 23.83201 & 8.923261 & 7.117947 & 18.54481 & 1837.147 & 1.309149 & 213.715 & 1.204999 \\
\hline 185356 & 388.356 & 156.4881 & 72.913 & 80.09522 & 124.0667 & 61.39426 & 3.44398 & 53.73184 & 17.3879 & 14.24923 & 31.1038 & 3803.635 & 2.674351 & 490.7871 & 1.79587 \\
\hline 42802 & 113.297 & 55.02459 & 996.0291 & 84.66234 & 91.06842 & 31.72919 & 40.26845 & 19.05463 & 9.098927 & 9.723478 & 22.71424 & 2246.984 & 2.195967 & 452.1661 & 2.108925 \\
\hline 96714 & 4560.921 & 300.8929 & 657.6613 & 139.0756 & 129.4553 & 34.75242 & 45.78313 & 48.35018 & 16.76962 & 4.067714 & 20.03977 & 3337.367 & 2.273123 & 463.9592 & 1.326137 \\
\hline 99898 & 5201.584 & 402.1105 & 60.8398 & 137.6994 & 110.5067 & 32.65863 & 41.37728 & 47.871 & 17.25849 & 4.749671 & 15.05689 & 4103.084 & 1.225817 & 532.3304 & 0.859748 \\
\hline 42922 & 7974.187 & 5.2799 & 659.31 & 106.0914 & 144.2021 & 38.74007 & 45.84387 & 50.48425 & 37.97885 & 6.015099 & 10.90816 & 8060.215 & 1.807431 & 81.9103 & 0.936277 \\
\hline 96252 & 6070.667 & 261.4787 & 666.7346 & 140.0862 & 151.592 & 45.33324 & 62.01074 & 57.35519 & 26.53236 & 7.147652 & 18.71941 & 5927.862 & 1.564249 & 55.5676 & 1.226368 \\
\hline 3.77837 & 533.382 & 3.8453 & 638.199 & 141.7003 & 199.4975 & 55.19325 & 7.9279 & 66.7298 & 33.61758 & 7.481864 & 17.45067 & 8513.494 & 1.738345 & 36.0037 & 0.928723 \\
\hline 013212 & 120.065 & 5.97799 & 82.7531 & 85.94753 & 91.94536 & 27.22073 & 6.2475 & 37.35319 & 11.42525 & 2.943609 & 13.7208 & 2582.373 & 1.781568 & 68.5537 & 0.682803 \\
\hline 106593 & 5021.858 & 78.75352 & 507.729 & 2.17912 & 175.0668 & 50.96165 & 59.87945 & 56.45399 & 25.81767 & 6.027366 & 18.94186 & 4725.29 & 0.932987 & 12.5152 & 0.719043 \\
\hline 997372 & 5370.219 & 1.06249 & 471.2193 & 59.61125 & 172.4566 & 48.88711 & 61.10956 & 44.41328 & 26.22855 & 5.030951 & 21.20816 & 4084.561 & 1.033437 & 269.3328 & 0.760633 \\
\hline 403675 & 013.433 & .70428 & 372.1287 & 84.27454 & 70.3218 & 25.66291 & 27.10995 & 20.91477 & 11.63213 & 2.193918 & 8.762705 & 2397.98 & 0.89962 & 61.6782 & 0.332398 \\
\hline 067394 & 19.881 & 167.0226 & 394.4828 & 92.2992 & 151.0389 & 24.60101 & 39.6805 & 22.82023 & 21.72362 & 9.268542 & 21.6269 & 5094.488 & 0.974656 & 18.9823 & 0.841496 \\
\hline 384425 & 3089.67 & .44397 & 3.9645 & 56.27135 & 105.0287 & 41.39158 & 38.04731 & 39.37139 & 15.60324 & 5.582271 & 16.54674 & 2598.948 & 1.172488 & 68.3488 & .008304 \\
\hline 415823 & 650.576 & 33.23955 & 359.0916 & 5.27603 & 136.3121 & 65.71905 & 58.25771 & 23.3267 & 17.47695 & 6.284372 & 13.20975 & 390.923 & 0.53472 & 23.7536 & .733238 \\
\hline 957597 & 553.945 & 102.558 & 373.5286 & 68.05435 & 143.4205 & 35.46726 & 37.41995 & 26.31794 & 18.33822 & 4.525088 & 8.873301 & 3333.902 & 0.780832 & 09.8867 & 1.723939 \\
\hline 145255 & 803.753 & 119.9514 & 88.8328 & 62.05324 & 266.6786 & 41.91473 & 39.91226 & 34.66677 & 23.77358 & 4.959063 & 9.799937 & 101.033 & 1.194654 & 9.3327 & .615382 \\
\hline 5.293023 & 7863.864 & 96.13032 & 865.0442 & 88.12017 & 264.2937 & 77.68821 & 100.9789 & 61.71913 & 31.07801 & 11.07537 & 25.16534 & 08.704 & 1.941974 & 2.7621 & 1.025899 \\
\hline 5.209819 & 7775.027 & 245.5629 & 535.5549 & 88.48698 & 221.8488 & 52.05804 & 62.77931 & 36.70396 & 23.72854 & 8.899814 & 28.16151 & 669.593 & 1.562836 & 13.2228 & 0.886225 \\
\hline 4.866335 & 7960.526 & 251.264 & 462.3396 & . & 79.773 & 14322 & 1.54039 & 28.10787 & 24.86738 & 13223 & 38.48406 & 45 & 2.116545 & 01 & 191 \\
\hline 6.288114 & 2072.807 & 44.22495 & 726.68 & 59.49768 & 69.71234 & 33.18488 & 38.81233 & 30.44161 & 9.655937 & 7.576103 & 29.76715 & 2014.146 & 1.086627 & 842 & 120105 \\
\hline 6.75286 & 175.623 & . 69649 & 700.853 & 57.7755 & 64.64216 & 41.5907 & 34.75922 & 32.69704 & 8.056883 & 6.454076 & 25.3839 & 1444.012 & 1.168206 & 6.0367 & 1.700014 \\
\hline 1.572721 & 2438.505 & 34.73259 & 175.9163 & 56.88371 & 76.04993 & 47.62268 & 44.94993 & 26.79173 & 12.97106 & 5.428445 & 7.278261 & 1479.933 & 1.236635 & 227.8667 & 0.819106 \\
\hline 4.910024 & 4140.278 & 48.08181 & 489.849 & 73.22104 & 86.76473 & 30.86509 & 37.90412 & 27.85666 & 13.72137 & 2.926735 & 15.6037 & 2225.798 & 0.554905 & 262.4558 & 0.695449 \\
\hline 629437 & 3658.842 & 737.7586 & 176.4084 & 97.80158 & 152.8819 & 81.10996 & 73.78095 & 40.01263 & 19.09262 & 4.067792 & 25.14236 & 198.774 & 1.460763 & 6.7733 & 0.884771 \\
\hline .262463 & 3951.177 & 598.1431 & 924.0769 & 91.3796 & 133.5202 & 21.66318 & 27.30371 & 25.45646 & 14.99874 & 11.69475 & 20.2508 & 5407.639 & 1.434821 & 302.0869 & 0.908867 \\
\hline 8.669586 & 5087.567 & 36.24885 & 1046.901 & 80.87944 & 71.47619 & 66.50119 & 51.96785 & 43.61718 & 14.257 & 6.331087 & 23.438 & 2164.078 & 1.377043 & 245.3312 & 1.377148 \\
\hline 217279 & 2393.618 & 29.02355 & 583.8426 & 53.23411 & 82.83834 & 30.5891 & 43.92022 & 28.38257 & 10.40476 & 10.07133 & 28.75559 & 1605.627 & 2.582004 & 343.763 & 1.442425 \\
\hline 229263 & 5243.269 & 122.8966 & 486.9807 & 156.5297 & 123.6458 & 42.43881 & 47.13481 & 43.30022 & 16.61342 & 4.337223 & 16.42091 & 3320.821 & 1.679628 & 491.505 & 0.61637 \\
\hline 723693 & 3921.999 & 389.804 & 71.1698 & 166.4728 & 21.678 & 42.10724 & 50.66435 & 52.34227 & 11.42391 & 1.809066 & 26.69017 & 500.429 & 1.15075 & 440.4711 & 0.881506 \\
\hline 827023 & 49.313 & 53.55496 & 736.5593 & .03524 & 80.81901 & 40.18016 & 5.32223 & 33.18115 & 10.91177 & 5.982096 & 19.24541 & 342.354 & 1.519037 & 40.8487 & 1.833714 \\
\hline 2.57851 & 8.641 & 8.4126 & 724.3867 & .6129 & 3.8279 & 27.94707 & 3.21817 & 43.09159 & 12.98364 & 14194 & 10.47419 & 81.284 & 0.994922 & 34.1691 & 1.099393 \\
\hline 374733 & 3.571 & 36.26832 & 52 & 68485 & .23301 & 26.74566 & 95 & 77 & 322 & 97 & 16.59451 & 944 & 1.0 & 7.4604 & 0.906956 \\
\hline .028018 & 13.807 & 51429 & 43 & 19227 & .42119 & 37 & 101 & & 12.05051 & 1788 & 22.18524 & 1869.92 & 2.543365 & 2.7509 & 1.179274 \\
\hline . 475771 & 2.567 & 30.67414 & .9033 & 30237 & 1.94277 & 2936 & 98397 & 6841 & 8.781989 & 55364 & 9.260794 & 1234.699 & 0.805058 & 0.239 & 2.118764 \\
\hline .545845 & 10.358 & .33054 & .3546 & 23311 & .79189 & 31.07902 & 40957 & 92047 & 10.16065 & 78132 & 18.15744 & 1981.23 & 1.591572 & 148.8789 & 0.953859 \\
\hline 728931 & 1.383 & 60752 & 3.3811 & 41528 & 6.5715 & 99017 & .78324 & 36081 & 17.39772 & 78207 & 27.56026 & 7.793 & 2.348978 & 459.532 & .834982 \\
\hline 327667 & 99.782 & 73411 & .2964 & 75.48749 & 18438 & 29.50931 & .42727 & 25.00717 & 13.52746 & 2.730123 & 9.114577 & 723.382 & 0.681968 & 8.0261 & 0.558873 \\
\hline 36396 & 3.654 & 8648 & 339.8259 & & 2.6254 & 49.32992 & 10563 & 77065 & 698688 & 71098 & 27653 & 6.965 & 1.377296 & 2.6787 & 9024 \\
\hline 1659 & 0.282 & 6802 & 133 & 96.94928 & 717 & 588 & 354 & 52 & 312 & 122 & 106 & 371 & . 194503 & 6.8338 & 0.872691 \\
\hline 26194 & 03.499 & 542 & 566 & 0.74 & & & 1514 & & & 03 & & & 892 & 01.9308 & \\
\hline
\end{tabular}


Crepis vesicaria s. taraxacifolia

Cruciata laevipes

Cuscuta epithymum

Cuscuta europaea

Cynosurus cristatus

Cytisus scopariu

Dactylis glomerata

Dactylorhiza incarnata

Dactylorhiza maculata s. fuchsii

Dactylorhiza majalis

Dactylorhiza majalis s. majalis

praetermiss

Danthonia decumbens

Daucus caro

Deschampsia cespitosa

Descurainia soph

Dianthus deltoides

Digitalis purpurea

Digitaria ischaemum

taxis tenuifolia

Dipsacus fullonum

Drosera rotundifolia

Dryopteris

Dryopteris carthusiana + D. dilatata

Dryopteris cristata

Dryopteris dilatat

Dryopteris filix-mas

Echinochloa crus-gall

Echinodorus ranunculoides

Echium vulgare

Eleocharis acicularis

Eleocharis multicauls

Eleocharis palustris

Eleocharis palustris ag. (incl. E. uniglumis

Eleocharis quinqueflora

eocharis uniglum

Elodea canadensi

Elymus caninus

Elytrigia atherica

Elytrigia juncea s. boreoatlantica

Elytrigia repens

Empetrum nigrum

Epilobium hirsutum

\begin{tabular}{|c|c|c|c|c|c|c|c|c|c|c|c|c|c|c|c|}
\hline 1.605165 & 3047.144 & 73.41998 & 277.6204 & 80.48742 & 84.49854 & 28.90892 & 32.4766 & 23.38813 & 11.2337 & 3.248054 & 8.840014 & 2303.283 & 0.595428 & 254.8896 & 0.496452 \\
\hline 1.59992 & 4225.259 & 59.92086 & 630.1695 & 77.35703 & 82.1559 & 37.20083 & 43.18668 & 42.17351 & 14.50556 & 6.963246 & 32.19957 & 2833.321 & 1.303622 & 340.5633 & 1.315819 \\
\hline 0.10111 & 2079.602 & 14.83458 & 1087.949 & 50.24753 & 81.9732 & 57.281 & 3.19213 & 31.63797 & 17.39607 & 6.293301 & 5.15231 & 2593.294 & 1.155843 & 282.1495 & 1.913495 \\
\hline 189603 & 589.204 & 170.4058 & 3.4715 & .07066 & 138.0376 & 46.49008 & 3.1418 & 49.56868 & 19.38911 & 5.796314 & 42.18573 & 4257.963 & 0.89937 & 525.4798 & 1.558632 \\
\hline 44019 & 688.828 & 352.6317 & 3.0627 & .03637 & 102.3803 & 27.49623 & 34.42652 & 23.80869 & 19.63114 & 3.824623 & 9.596672 & 4098.706 & 0.560224 & 372.0475 & 0.803918 \\
\hline 21992 & 072.909 & 33.8318 & 78.4472 & 73.57464 & 101.376 & 55.99657 & 76.53605 & 37.62547 & 15.48261 & 14.50159 & 13.61879 & 2743.594 & 3.626102 & 394.8594 & 1.355716 \\
\hline 42967 & 932.269 & 89.94947 & 0.1604 & 113.2589 & 106.6825 & 39.51138 & 48.33501 & 46.00626 & 14.33051 & 6.332904 & 21.52107 & 3433.364 & 1.748635 & 387.9114 & 1.54959 \\
\hline 54739 & 963.428 & 423.0806 & 415.9139 & 96.1307 & 164.8282 & 30.33141 & 32.45674 & 29.62312 & 21.63409 & 5.302565 & 12.28984 & 5557.532 & 1.053583 & 428.7394 & 1.21555 \\
\hline 26571 & 195.865 & 54.78659 & 524.4651 & 82.58532 & 203.6047 & 62.05109 & 85.82895 & 35.128 & 37.88311 & 4.687688 & 12.23357 & 6505.261 & 0.796501 & 359.9048 & 1.862041 \\
\hline 91967 & 741.754 & 412.3078 & 359.1485 & 60.35142 & 111.1643 & 45.55442 & 39.87213 & 52.46897 & 8.989393 & 4.898836 & 25.44827 & 1574.902 & 0.505641 & 139.1046 & 1.384718 \\
\hline 320547 & 554.146 & 188.4589 & 407.3042 & 108.8021 & 227.0827 & 27.81462 & 34.89702 & 25.54924 & 24.1886 & 7.004159 & 11.875 & 5096.268 & 0.838093 & 564.4473 & 1.131831 \\
\hline 42674 & 5295.864 & 186.7997 & 394.9493 & 68.89152 & 203.7406 & 25.86188 & 31.68144 & 24.23983 & 17.02013 & 8.020478 & 12.11776 & 3698.167 & 0.760336 & 522.2995 & 0.942661 \\
\hline 064821 & 54.648 & 244.8473 & 37.5683 & 86.62891 & 201.6779 & 31.34476 & 39.53014 & 24.29548 & 20.8755 & 8.299739 & 14.93042 & 4612.162 & 1.400843 & 740.7629 & 0.914929 \\
\hline 196607 & 788.257 & 51.60159 & 764.1125 & 93.27769 & 210.5525 & 79.92006 & 111.6452 & 37.021 & 33.2846 & 6.208526 & 9.428508 & 6496.719 & 1.021279 & 18.6002 & 1.361984 \\
\hline 199672 & 95.964 & 514.0594 & 430.4209 & 119.0977 & 124.603 & 50.2237 & 53.12058 & 31.6643 & 20.56548 & 4.697859 & 12.90489 & 3720.278 & 0.863562 & 455.0162 & 0.681857 \\
\hline 009509 & 86.881 & 81.51794 & 01.5424 & 138.8814 & 157.2921 & 60.54613 & 76.27825 & 48.37887 & 23.58285 & 9.42576 & 29.03973 & 4020.967 & 2.104566 & 316.7458 & 1.282579 \\
\hline 153575 & 549.836 & 32.24999 & 01.5422 & 50.41953 & 74.56554 & 54.15045 & 67.55507 & 40.88267 & 13.69944 & 11.38192 & 12.79259 & 3175.314 & 2.577817 & 635.7905 & 1.08066 \\
\hline 537692 & 31.317 & 145.7849 & 376.191 & 159.5883 & 148.6431 & 58.77936 & 53.10083 & 41.23949 & 10.13198 & 2.440828 & 24.80547 & 150.225 & 0.881008 & 339.893 & 0.614733 \\
\hline 959002 & 968.43 & 28.15191 & 85.5545 & 64.46695 & 76.04078 & 39.52607 & 48.79827 & 26.72046 & 15.68171 & 14508 & 6.870469 & 2629.048 & 0.996509 & 91.0947 & 0.677541 \\
\hline 6.26947 & 343.418 & 35.49286 & 42.8202 & 66.17524 & 103.4058 & 52.47985 & 1.14216 & 37.37937 & 14.2704 & 368935 & 26.94439 & 2221.366 & 2.377582 & 68.2147 & 1.363068 \\
\hline 4.389178 & 54.841 & 99.5272 & 336.985 & 3.6061 & 139.9184 & 49.41701 & 68.68618 & 59.60401 & 16.40856 & 679326 & 14.10048 & 348.05 & 3.679893 & 70.5151 & 1.027167 \\
\hline .238556 & 64.538 & 103.4338 & 270.5171 & 137.8889 & 145.5677 & 55.62795 & 53.5349 & 39.75989 & 15.59376 & 228629 & 18.67442 & 2659.367 & 1.794294 & 76.852 & 0.696534 \\
\hline 1.858807 & 4932.009 & 295.008 & 566.7013 & 118.8918 & 106.2481 & 47.72423 & 51.63844 & 49.13229 & 13.61237 & 7.333835 & 25.88199 & 2781.645 & 1.495613 & 257.9692 & 1.302888 \\
\hline .349324 & 920.689 & 12.51413 & 679.9062 & 65.82978 & 162.2324 & 49.36119 & 51.47834 & 29.96514 & 19.51993 & 6.926814 & 5.707271 & 3672.803 & 1.420812 & .4187 & 0.822517 \\
\hline 399325 & 681.614 & 144.1012 & 470.9052 & 83.81258 & 324.4431 & 63.14348 & 95.14058 & 27.5298 & 26.35308 & 10.86279 & 10.23452 & 4837.495 & 2.690934 & 1.1877 & 0.876854 \\
\hline .440006 & 3944.849 & 82.40374 & 563.1496 & 87.9759 & 202.8712 & 80.35162 & 103.9104 & 51.152 & 23.42151 & 8.54531 & 31.79758 & 4151.007 & 2.094431 & 636 & .484276 \\
\hline 79211 & 32.035 & 290.9772 & 2.0048 & 109.3609 & 321.7202 & 110.318 & 142.8751 & 88.49807 & 34.45898 & 9.93025 & 39.9347 & 5454.292 & 2.892685 & 381.9678 & 1.310516 \\
\hline . 880109 & 6540.498 & 127.439 & 470.3445 & 79.05973 & 262.2131 & 55.20933 & 70.85152 & 27.40097 & 22.70777 & 9.063206 & 24.74996 & 3305.299 & 1.800763 & 615.593 & 0.831722 \\
\hline .722932 & 3328.307 & 63.22945 & 537.6411 & 78.37346 & 141.7596 & 67.13654 & 87.92986 & 47.98295 & 19.66456 & 8.283106 & 29.21881 & 3534.977 & 2.066872 & 464.5304 & 1.448027 \\
\hline 173049 & 3198.828 & 65.82793 & 855.4837 & 66.74958 & 105.2576 & 46.72411 & 57.19486 & 41.61561 & 15.23075 & 9.917562 & 27.4877 & 3094.861 & 1.998649 & 1694 & 1.371914 \\
\hline .550341 & 5116.578 & 118.8398 & 650.1808 & 114.8305 & 158.1022 & 54.36306 & 84.36987 & 70.15497 & 16.74711 & 3.11446 & 14.7586 & 3389.105 & 2.514627 & 335.8497 & 0.884701 \\
\hline 1.574697 & 5636.098 & 432.5795 & 629.0335 & 105.6314 & 184.0995 & 15.04558 & 28.67894 & 16.24626 & 15.16715 & 5.413244 & 13.30562 & 4019.268 & 0.705359 & 494.4782 & 1.169138 \\
\hline 103812 & 3128.641 & 65.50281 & 259.0782 & 97.51492 & 111.115 & 53.40455 & 45.66348 & 32.08744 & 13.51104 & 5.460085 & 21.51026 & 1993.153 & 1.243591 & 337.1174 & 0.670694 \\
\hline 106085 & 5904.563 & 201.0097 & 8.5272 & 100.0244 & 207.5007 & 32.87521 & 48.30101 & 36.6075 & 24.27706 & 6.712407 & 22.16583 & 125.638 & 1.074159 & 594.8893 & 1.429059 \\
\hline 918696 & 6800.833 & 1.7037 & 5.5917 & 0.44369 & 285.4146 & 14.38536 & 23.58019 & 15.9546 & 19.98846 & 10.79865 & 11.26773 & 370.934 & 1.450967 & 342.0172 & 1.039475 \\
\hline .655194 & 34.821 & 372.2524 & .7992 & 102.6917 & 179.5738 & 31.50796 & 52.40628 & 33.25775 & 22.6362 & 5.174258 & 17.07671 & 88.936 & 1.04158 & 762.908 & 1.135285 \\
\hline .746944 & 2.892 & 6.2801 & .9018 & 33 & 2.2658 & 35.46952 & 3.8269 & 38.49278 & 25.02526 & 10.99815 & 18.02192 & 54.261 & 1.174653 & 69.5392 & 1.460187 \\
\hline 5.39689 & 30.653 & 54 & 5.7808 & 106. & 438 & 38 & 2.64662 & 34.36444 & 14.879 & 4.796922 & 12.14694 & 151.907 & 038 & 69 & . 47224 \\
\hline .832369 & 5342.065 & 2906 & 69 & 2.533 & 7.2242 & 37.41627 & 5417 & 40.46506 & 15.39499 & 91956 & 15.49322 & 941.75 & 0.982294 & 5.9609 & 1.363945 \\
\hline .380713 & 8.471 & .8486 & 1513 & 77.26351 & 3.2801 & 20.4718 & 1.76482 & 41025 & 1461 & 16471 & 12.3696 & 4367.919 & 0.976608 & 7.8351 & 0.648452 \\
\hline .281703 & 540.973 & .91407 & 6.6485 & 86.44951 & 49.668 & 106.4252 & 19.3242 & 17165 & 38.43689 & 6.941595 & 37.12195 & 8178.546 & 1.841671 & 67.5249 & 1.135595 \\
\hline .374302 & 74.197 & 733.5114 & 0.0693 & 19 & 6.7211 & 698 & 48599 & 43.97317 & 25.89829 & 4231 & 27.04362 & 3259.44 & 1.670722 & 2.8299 & 0.981682 \\
\hline 1.21645 & 66.267 & 8.9131 & .2282 & 130.487 & 4.9085 & 86 & 62391 & 69.73889 & 21.34745 & 88916 & 26.57148 & 2263.252 & 1.2052 & .3868 & .96158 \\
\hline 030006 & 61.955 & 561.0629 & 583.1749 & 017 & 127.3922 & 837 & 54 & 47.49689 & 43397 & 69849 & 17.21418 & 128 & 1.754976 & 0.4819 & 401679 \\
\hline 0.31234 & 0.422 & 130.8232 & 5.189 & & 132 & & 21 & 27 & 534 & & 619 & .752 & . .369591 & 7919 & 225 \\
\hline 1015 & 96.957 & & 97 & 338 & 174 & 266 & 136 & 68 & 308 & & 847 & & & 90.032 & \\
\hline
\end{tabular}


Epilobium montanum

Epilobium obscurum

Epilobium palustre

Epilobium parviflorum

Epilobium roseu

Epilobium tetragonum

Epipactis helleborine

Epipactis palustris

Equisetum fluviatile

Equisetum hyemale

Equisetum hyemale ag. (incl. E. x moorei)

Equisetum palustre

Equisetum telmateia

Equisetum variegatum

Equisetum x litorale

Erica tetralix

Eriophorum angustifolium

Eriophorum vaginatum

Erodium cicutarium s. cicutariu

Erodium cicutarium s. dunense

Erodium lebelii

Erophila verna

Eryngium campestre

Eryngium maritimum

Erysimum cheiranthoides

Euonymus europaeus

Euphorbia cyparissias

Euphorbia esula s. esula

Euphorbia exigua

Euphorbia helioscopia

Euphorbia palustris

Euphorbia peplus

Euphrasia stricta

Fagus sylvatica

Fallopia convolvulus

Fallopia dumetoru

arenaria

Festuca arundinacea

Festuca cinerea

Festuca filiformis

Festuca gigantea

Festuca ovin

Festuca ovina ag. (incl. F. cinerea, F. filiformis)

Festuca pratensis

\begin{tabular}{|c|c|c|c|c|c|c|c|c|c|c|c|c|c|c|c|}
\hline .378277 & 4162.334 & 160.8415 & 682.5986 & 81.3305 & 178.7524 & 62.66458 & 69.72017 & 53.48473 & 23.28016 & 5.814485 & 22.76007 & 4757.738 & 1.744376 & 474.196 & 1.401101 \\
\hline 322686 & 8627.893 & 370.5921 & 740.7089 & 127.9589 & 314.5449 & 68.91666 & 98.85291 & 73.14981 & 36.93058 & 13.21198 & 29.64958 & 9367.492 & 1.872545 & 892.5858 & 1.650401 \\
\hline 107519 & 332.127 & 436.8035 & 501.8284 & 132.151 & 209.7254 & 45.68455 & 67.4857 & 34.17302 & 25.71108 & 10.70715 & 30.47271 & 5358.538 & 1.593372 & 817.2266 & 1.081701 \\
\hline 148081 & 8474.201 & 1.6261 & 7.1205 & 140.0971 & 264.2156 & 54.51369 & 66.12491 & 57.64951 & 31.34991 & 9.173115 & 25.0449 & 7401.156 & 1.104494 & 667.9004 & 1.555967 \\
\hline 81691 & 156.364 & 0.4128 & 4.8706 & 111.8646 & 286.5607 & 52.83956 & 5.88267 & 52.91138 & 39.24255 & 9.310587 & 25.86638 & 8422.245 & 2.889221 & 824.4634 & 2.419723 \\
\hline 47398 & 8256.739 & 504.5509 & 6.4966 & 129.1223 & 238.1432 & 52.84229 & 68.88113 & 47.77815 & 33.14686 & 10.59293 & 31.96819 & 6554.481 & 1.792688 & 584.1493 & 1.953931 \\
\hline 7.11857 & 203.304 & 79.96424 & 1012.175 & 92.23751 & 138.7484 & 46.64476 & 56.34499 & 44.24015 & 21.45087 & 7.498797 & 33.26665 & 3678.778 & 1.921438 & 456.5047 & 1.884588 \\
\hline .440088 & 34.795 & 460.0924 & 409.4248 & 89.48903 & 181.9759 & 42.24364 & 45.83096 & 36.14927 & 21.18 & 4.539877 & 12.2774 & 5582.024 & 0.979439 & 340.9198 & 1.375087 \\
\hline 36232 & 692.177 & 223.2327 & 653.7975 & 123.187 & 127.6444 & 45.96203 & 56.03752 & 51.0737 & 21.36792 & 7.998234 & 22.52394 & 4316.669 & 1.692788 & 402.7852 & 1.500239 \\
\hline 906374 & 18.721 & 196.0535 & 446.1835 & 125.3557 & 183.2452 & 35.53631 & 49.71628 & 33.46428 & 20.03347 & 10.76732 & 26.3727 & 5788.242 & 1.523322 & 565.8358 & 0.943 \\
\hline 538429 & 5069.976 & 92.84252 & 695.735 & 83.44566 & 199.5582 & 61.19438 & 78.77084 & 55.56703 & 25.38568 & 11.78823 & 23.8181 & 4967.403 & 2.798112 & 285.6612 & 1.676277 \\
\hline 457543 & 6886.617 & 113.9491 & 812.7121 & 91.15763 & 176.9031 & 69.51579 & 83.6631 & 87.2905 & 30.95232 & 11.56159 & 33.7257 & 6479.826 & 1.905844 & 376.2626 & 2.167222 \\
\hline 74434 & 241.536 & 202.0101 & 02.3304 & 130.7414 & 232.4272 & 46.09006 & 64.00626 & 37.96198 & 25.13774 & 7.42732 & 25.07612 & 6907.397 & 1.377981 & 51.0934 & 1.154211 \\
\hline 906101 & 004.892 & 97.51519 & 357.2382 & 88.35636 & 156.5559 & 47.85992 & 51.13955 & 41.04078 & 21.40837 & 7.910921 & 23.66241 & 3491.912 & 0.88076 & 65.8578 & 0.908772 \\
\hline 577292 & 7424.289 & 226.6674 & 385.657 & 114.9955 & 171.3967 & 34.87175 & 39.65453 & 26.46393 & 21.49589 & 3.788419 & 11.82542 & 4996.95 & 0.840651 & 393.791 & 0.866291 \\
\hline 352125 & 811.817 & 248.9076 & 880.0624 & 150.0664 & 306.6408 & 75.96409 & 105.0738 & 63.1964 & 33.18701 & 12.83232 & 34.55596 & 8669.531 & 2.678903 & 904.3194 & 1.581599 \\
\hline 8.63517 & 3699.55 & 30.1681 & 1016.644 & 73.85474 & 152.5898 & 99.13974 & 153.4974 & 49.51198 & 23.60568 & 9.214962 & 7.421564 & 4190.575 & 2.526333 & 96.2025 & 1.096115 \\
\hline . 179141 & 3074.619 & 323.5069 & 228.2937 & 78.99614 & 120.2872 & 54.68211 & 54.81224 & 38.37068 & 16.8236 & 5.100509 & 10.67226 & 1866.014 & 1.25312 & 1.1032 & 1.255892 \\
\hline 0.16623 & 689.521 & 128.0812 & 511.065 & 116.4576 & 308.6559 & 44.61427 & 55.37794 & 32.69897 & 31.49994 & 10.08183 & 10.74901 & 995.28 & 2.433816 & 29.7323 & 1.076565 \\
\hline 617206 & 804.523 & 24.019 & 631.8102 & 68.52153 & 154.3922 & 102.1748 & 163.8993 & 38.64738 & 22.40465 & 9.865187 & 6.61839 & 5319.239 & 1.853866 & 8.4624 & 0.628088 \\
\hline 161318 & 4018.497 & 92.70434 & 298.1212 & 128.7932 & 124.2715 & 37.17882 & 47.06074 & 30.40635 & 11.96285 & 2.819411 & 13.08132 & 2352.866 & 2.845273 & 09.3341 & 1.185093 \\
\hline .858748 & 2552.846 & 47.37191 & 171.7156 & 57.16727 & 97.52125 & 61.39563 & 54.02949 & 23.992 & 11.42564 & 248637 & 11.84917 & 1473.805 & 1.436398 & 62.4013 & 0.779235 \\
\hline .646596 & 1783.139 & 47.59089 & 154.5581 & 41.35399 & 80.1083 & 52.26375 & 45.34769 & 25.11016 & 9.558613 & 4.170288 & 14.02434 & 1341.07 & 1.401105 & 247.481 & .042326 \\
\hline 1.590298 & 3677.806 & 52.368 & 158.0873 & 74.57972 & 114.3909 & 70.55659 & 62.45183 & 37.33736 & 13.89281 & 349667 & 10.36597 & 2030.982 & 2.018544 & 325.2377 & 19645351 \\
\hline 1.832047 & 4726.959 & 67.30345 & 288.3801 & 123.9283 & 128.4276 & 44.23349 & 48.77379 & 27.33849 & 15.81388 & 08387 & 12.32269 & 13.398 & 1.240173 & 54 & 99 \\
\hline 1.00011 & 3343.513 & 726.8226 & 90.39604 & 92.97531 & 173.27 & 82.07349 & 72.48267 & 45.44295 & 13.23089 & 83537 & 19.94915 & 503.318 & 1.016129 & 192 & 563 \\
\hline 3.14404 & 346.135 & 4.1292 & 626.6097 & 122.3155 & 125.6328 & 32.17304 & 2.87317 & 44.09952 & 18.33686 & 3.734181 & 22.52998 & 3718.693 & 2.397907 & 80.7797 & 0.769111 \\
\hline 5.508424 & 2627.57 & 39.90879 & 811.4726 & 64.45122 & 87.65211 & 33.20646 & 36.47332 & 27.43888 & 14.87206 & 5.521526 & 24.84439 & 2756.565 & 1.012821 & 11.0219 & 1.484497 \\
\hline 186074 & 3749.946 & 48.16887 & 367.2521 & 88.60335 & 89.75698 & 46.56395 & 51.43987 & 41.83612 & 15.1495 & 5.327353 & 19.24674 & 2463.78 & 1.546503 & 43.5842 & 0.77993 \\
\hline .220239 & 3818.526 & 111.4196 & 374.7437 & 96.02609 & 99.68096 & 31.54562 & 35.41719 & 26.82411 & 14.28419 & 4.418275 & 14.69266 & 3388.773 & 0.934431 & 37.1545 & 0.507354 \\
\hline 742772 & 2775.194 & 82.02628 & 610.671 & 119.7832 & 72.37698 & 26.25367 & 28.74245 & 32.47875 & 11.32048 & 2.810875 & 8.765665 & 2075.265 & 1.965946 & 49.9548 & 0.430535 \\
\hline 555683 & 3215.79 & 87.72593 & 564.1499 & 114.0705 & 93.92975 & 22.89779 & 28.10589 & 39.85119 & 12.55997 & 3.587623 & 15.7704 & 2286.177 & 1.504467 & 73.7725 & 0.651296 \\
\hline 698459 & 8121.217 & 112.0864 & 408.9807 & 107.2642 & 208.3056 & 48.48887 & 66.0668 & 32.21758 & 28.54478 & 11.15513 & 26.60131 & 4496.555 & 1.236112 & 362.4889 & 1.449241 \\
\hline 598993 & 3962.019 & 108.9242 & 963.9962 & 134.1315 & 3.13164 & 25.44956 & 35.64998 & 47.07241 & 13.08105 & 3.184677 & 27.91043 & 2992.422 & 1.349568 & 518.4427 & 0.690189 \\
\hline 671383 & 6923.296 & 4.1664 & 368.4439 & 108.1854 & 285.1402 & 78.1914 & 80.8212 & 48.05714 & 26.647 & 5.46509 & 11.58401 & 4433.906 & 0.756409 & 44.0104 & 1.393336 \\
\hline 239445 & 2468.439 & 38194 & 484.215 & 5.51629 & 99.27289 & 56.19559 & 75.83315 & 30.44622 & 16.72765 & 9.863712 & 32.51959 & 17.594 & 2.385597 & 33.6541 & 1.38966 \\
\hline 23464 & 0.177 & 0347 & 687.7007 & 6.2412 & 4.7847 & 44.95742 & 56.97557 & 37.09173 & 11.02907 & 701 & 19.79509 & 10.789 & 2.596561 & 9.8739 & 1.605372 \\
\hline 43095 & 9.033 & 55.23013 & 07 & 65.96351 & 79 & 12906 & 837 & 33.66872 & 14.48685 & 74 & 21.50583 & 726 & 2.488178 & 112 & 1.919376 \\
\hline 52434 & 17.255 & 2029 & 1.9546 & 11716 & 109.4855 & 74.30967 & 2735 & 2718 & 12.78317 & 1407 & 17.77169 & 7.322 & 1.839347 & 3.2212 & 1.582562 \\
\hline 372098 & 43.484 & . .6782 & 9.6778 & .0871 & 9.2917 & 15183 & 46.84401 & 5275 & 18.62667 & 5.508997 & 15.64406 & 67.786 & 0.993082 & 62.0166 & 1.010978 \\
\hline 1.591305 & 3733.547 & 02912 & 88.6349 & 89.68852 & 5.2325 & 58.41281 & 72.42934 & 35.48901 & 13.85757 & 238 & 10.99509 & 46.957 & 1.921378 & 9.7437 & 1.408427 \\
\hline 10.19047 & 2813.03 & 54824 & 1.8822 & 66516 & 97.98413 & 1187 & 3.28037 & 46.10706 & 13.83417 & 89547 & 11.87435 & 240.987 & 1.779487 & 9.4045 & 2.058686 \\
\hline .585037 & 3630.879 & 55689 & .8882 & 09916 & 8.823 & 38.02142 & .18275 & 37.08319 & 1684 & 48653 & 13.4907 & 591.381 & 1.062288 & 8.4478 & .012104 \\
\hline 165093 & 00.457 & 29702 & 2844 & 92.85738 & 122.0737 & 63.398 & 65596 & 3 & 838 & 21413 & 11.32403 & 12.136 & 1.234123 & 8.4132 & 459099 \\
\hline 77 & .666 & 17 & & , & 748 & & 202 & & & & 5301 & 265 & 35583 & 3.7925 & 1.880768 \\
\hline 8826 & & 136.829 & & 21 & & 51165 & .77816 & 995 & 507 & & & & .702279 & 36 & \\
\hline
\end{tabular}


Festuca rubra

Festuca rubra ag. (incl. F. arenaria) Festulolium loliaceu

Filago minima

Filipendula ulmaria

Fraxinus excelsio

Fritillaria meleagris

Fumaria officinali

Galanthus nivalis

Galeopsis bifida

Galeopsis bifida + G. tetrahi

Galeopsis speciosa

Galeopsis tetrahi

Galium aparine

Galium mollugo

Galium odoratum

Galium palustre

Galium pumilun

Galium saxatile

Galium uliginosum

Galium verum

enista anglica

Genista pilosa

Gentiana cruciata

umonan

Gentianella amarella

Gentianella germanica

Geranium dissect

Geranium molle

Geraium premicto

Geranium robertianu

Geum urbanum

Glaux maritima

Glechoma hederacea

Glyceria declinata

Glyceria fluitans

Gnaphalium luteo-album

Gnaphalium sylvaticum

Gnaphalium uliginosum

Groenlandia densa

Gymnadenia conopsea

Hammarbya paludosa

Hedera helix

\begin{tabular}{|c|c|c|c|c|c|c|c|c|c|c|c|c|c|c|c|}
\hline 282286 & 551.495 & 749.5521 & 384.6843 & 162.6031 & 204.5135 & 72.94059 & 81.02941 & 43.94263 & 32.35726 & 6.766166 & 15.10773 & 5416.084 & 1.43298 & 524.2703 & 1.788072 \\
\hline 523933 & 433.388 & 750.0438 & 430.295 & 148.4422 & 215.0129 & 73.21249 & 85.02147 & 44.13339 & 33.03673 & 7.762431 & 16.13069 & 6293.077 & 1.33182 & 568.8376 & 2.169 \\
\hline 165521 & 672.467 & 93.89134 & 363.1202 & 129.1123 & 140.1064 & 36.81037 & 45.52169 & 36.81312 & 31.91292 & 4.812014 & 20.98089 & 4640.183 & 1.274052 & 527.6917 & 1.197696 \\
\hline 89764 & 2762.98 & 20.94994 & 3.4747 & 3.12077 & 74.19111 & 46.74082 & .21602 & 36.45129 & 12.56396 & 2.634837 & 9.170448 & 1982.705 & 0.538119 & 310.6511 & 0.962716 \\
\hline 65481 & 7415.519 & 3.3238 & 503.041 & 1.0281 & 218.9433 & 54.4183 & 66.64644 & 44.17038 & 26.05378 & 7.735989 & 9.59973 & 4815.204 & 1.469769 & 460.6144 & 0.844 \\
\hline 46479 & 3367.59 & 59.37102 & 613.9301 & 5.26924 & 116.645 & 46.06962 & 46.67988 & 41.67751 & 15.409 & 7.895249 & 9.25523 & 2952.498 & 2.156871 & 271.5625 & 1.489858 \\
\hline 34717 & 7527.578 & .28748 & 9.3781 & 93.59812 & 95.37819 & 44.89846 & 54.26374 & 54.84478 & 25.89056 & 4.533845 & 29.90238 & 5080.234 & 0.891287 & 277.7597 & 0.541242 \\
\hline 14784 & 3761.865 & 139.7439 & 3.3545 & 127.1482 & 33.18939 & 30.03573 & 35.9104 & 42.75865 & 12.36353 & 3.46188 & 15.3955 & 2367.311 & 1.817054 & 345.0444 & 0.75971 \\
\hline 13366 & 2727.412 & 32.10751 & 1.8098 & 54.82256 & 62.62757 & 30.80842 & 31.43115 & 14.61363 & 8.685701 & 5.979834 & 18.19432 & 1173.827 & 1.286576 & 199.2491 & 0.982873 \\
\hline 38005 & 7248.835 & 116.1242 & 70.4535 & 106.827 & 292.2233 & 87.25943 & 122.852 & 65.31906 & 39.21315 & 15.74192 & 26.66994 & 5833.531 & 2.059544 & 570.4532 & 1.562126 \\
\hline 698862 & 6166.824 & 97.30997 & 1.3052 & 119.1477 & 306.0218 & 88.02321 & 119.8158 & 62.04749 & 39.67691 & 7.223884 & 25.3532 & 4452.503 & 1.902367 & 279.0789 & 1.834581 \\
\hline 89366 & 4680.648 & 99.56043 & 74.318 & 104.0614 & 191.7077 & 43.95171 & 57.16269 & 48.38319 & 29.20367 & 8.612928 & 33.43074 & 4934.405 & 2.606704 & 64.1614 & 1.583619 \\
\hline 37331 & 4204.671 & 3.90158 & 697.6877 & 89.3841 & 160.9714 & 54.97091 & 79.07627 & 48.24554 & 22.47543 & 13.09358 & 27.60938 & 3780.199 & 2.610949 & 318.4686 & 1.701787 \\
\hline 6.05582 & 165.033 & 146.4205 & 749.5849 & 86.21346 & 151.0965 & 47.47031 & 53.5489 & 45.9023 & 23.22577 & 9.589931 & 21.18092 & 4539.394 & 1.338073 & 451.1929 & 1.69568 \\
\hline 975424 & 4945.283 & 62.83214 & 408.0366 & 120.8215 & 137.5703 & 71.66669 & 69.83054 & 37.1995 & 22.14933 & 6.598121 & 19.57266 & 2982.137 & 1.497159 & 402.5027 & 1.263511 \\
\hline 547805 & 97.108 & .71849 & 352.6405 & 38.5049 & 64.45739 & 27.33934 & 23.54855 & 21.83069 & 8.045657 & 4.171108 & 16.6364 & 808.1938 & 2.496973 & 133.6901 & 0.838322 \\
\hline 281365 & 951.382 & 273.6938 & 494.429 & 124.283 & 221.2278 & 45.20909 & 65.53515 & 41.01984 & 24.33333 & 10.08216 & 28.65699 & 5356.309 & 1.440026 & 82.478 & 0.991921 \\
\hline 042407 & 94.241 & 17.69918 & 273.3682 & 67.16225 & 107.3175 & 55.7706 & 41.84747 & 22.78658 & 9.450766 & 4.053197 & 9.896028 & 1731.505 & 0.481403 & 245.462 & 0.371083 \\
\hline 9.05393 & 08.075 & 29.98457 & 1060.031 & 1.96762 & 78.31231 & 64.80647 & 80.78113 & 42.25779 & 14.87859 & 1.11851 & 9.733144 & 2718.521 & 3.199183 & 78.6209 & 1.327421 \\
\hline 672766 & 6672.83 & 351.388 & 421.7122 & . 08589 & 187.2085 & 32.3575 & 39.82697 & 23.6526 & 21.03266 & 5.746968 & 13.12969 & 4467.982 & 1.058682 & 66.9006 & 0.79125 \\
\hline 509617 & 735.847 & 44.50149 & 223.3004 & 83.93223 & 119.2759 & 68.10183 & 60.76437 & 30.44406 & 15.27234 & 4.527398 & 11.51421 & 2145.128 & 1.533563 & 42.9337 & 1.14702 \\
\hline .232192 & 3068.625 & 21.4409 & 1061.224 & 69.48905 & 117.8057 & 80.79155 & 103.12 & 36.37136 & 19.50553 & 753936 & 6.740259 & 3616.975 & 1.352152 & 6.2508 & . 978433 \\
\hline .346006 & 1545.023 & 15.56368 & 1105.198 & 44.77139 & 73.89513 & 62.73295 & 80.0138 & 40.37368 & 14.9994 & 7.712072 & 5.60138 & 2247.482 & 1.329956 & 7.7305 & 0.916127 \\
\hline .758593 & 4396.686 & 61.44713 & 0.6347 & 66.09252 & 129.2364 & 57.79523 & 70.32291 & 32.27085 & 24.55221 & 3.588464 & 10.58933 & 3177.327 & 0.705323 & 50.2056 & $9 / 5332$ \\
\hline .315932 & 1958.218 & 55473 & 1.0429 & 5.19481 & 69.98127 & 37.066 & 40.6515 & 32.64927 & 12.77382 & 172471 & 11.30558 & 886.251 & 1.18581 & 14 & 802808 \\
\hline 1.159353 & 5689.491 & 40.39817 & 4.0819 & 68.23224 & 182.1934 & 68.60798 & 83.04363 & 35.79008 & 27.7682 & 4.791059 & 7.864783 & 4997.251 & 1.356047 & 8.9721 & 0.930378 \\
\hline 876692 & 4990.558 & 505.3345 & 2.9069 & 80.20369 & 151.8777 & 42.3596 & 40.64619 & 37.0476 & 12.32006 & 4.063352 & 8.249934 & 82.652 & 0.846434 & .2114 & 0.796616 \\
\hline 990183 & 3101.317 & 36.31332 & 458.1009 & 66.28468 & 75.03879 & 33.82895 & 32.8921 & 24.36115 & 6.850015 & 2.169591 & 11.38054 & 1506.468 & 0.474644 & 219.9581 & 0.400608 \\
\hline . 491931 & 3621.302 & 225.248 & 481.6494 & 92.47471 & 78.8042 & 26.85145 & 30.32948 & 32.74193 & 15.16553 & 3.940076 & 10.78897 & 2983.022 & 1.306106 & 73.3437 & 0.536189 \\
\hline .115296 & 4738.397 & 248.3438 & 322.7071 & 143.9572 & 130.5301 & 59.24774 & 58.21385 & 30.66816 & 14.44729 & 3.766567 & 13.99599 & 302.599 & 1.358144 & 408.0276 & 0.785505 \\
\hline .138636 & 4072.927 & 86.58088 & 426.0483 & 115.079 & 113.3806 & 33.26782 & 40.22534 & 39.22265 & 13.42499 & 3.385243 & 16.00956 & 727.031 & 1.955594 & 494.776 & 0.937791 \\
\hline 470514 & 6909.4 & .13854 & 619.1924 & 84.47059 & 85.10015 & 33.16279 & 40.1011 & 47.16709 & 11.68571 & 4.373876 & 23.46142 & 2162.719 & 0.980332 & 253.3948 & 1.206381 \\
\hline .716787 & 3076.59 & 51.18836 & 732.2273 & 62.90155 & 94.06108 & 37.56711 & 36.08149 & 27.66411 & 14.61931 & 5.286509 & 17.50164 & 3322.832 & 0.98366 & 406.6611 & 1.177161 \\
\hline 613487 & 2815.528 & 45.02096 & 9.1479 & .74157 & 84.92804 & 35.30528 & 33.19995 & 26.45044 & 13.36494 & 4.943237 & 16.87888 & 3049.738 & 0.917162 & 364.1761 & 1.118043 \\
\hline 893974 & 5332.892 & 5.9772 & 83.7031 & 99.54583 & 153.7639 & 40.3927 & 30.54524 & 46.85298 & 14.60606 & 489871 & 11.85486 & 138.672 & 0.844619 & 73.3685 & 0.879715 \\
\hline 290149 & 6210.873 & 29017 & 1.6208 & 7.2852 & 139.8333 & 53.35623 & 65.29051 & 51.81058 & 24.31925 & 7.060634 & 25.63656 & 218.443 & 1.567981 & 1.9659 & 1.472313 \\
\hline 066909 & 7290.29 & .3957 & 5.2573 & 1333 & 175.6175 & 50.51092 & ..56394 & 50.38038 & 25.20058 & 11.11294 & 29.3999 & 7909.009 & 1.103301 & 33.6709 & 1.673945 \\
\hline 197005 & 212.52 & 1543 & .0182 & 098 & 05 & 44.62894 & 189 & 9302 & 126 & 65 & 18.22786 & 76 & 1.307889 & 048 & 1.078925 \\
\hline 99705 & 5593.821 & 6624 & 7.2017 & 2292 & 34 & 53.1075 & 158 & 02873 & 581 & 01666 & 24.84615 & 25.253 & 1.505419 & 5.4555 & 0.967141 \\
\hline . 104105 & 4949.137 & 5.1425 & .7667 & 7172 & 4.9984 & .52583 & 0.84599 & 80892 & 4.45157 & 6.932146 & 22.69044 & 66.722 & 1.488319 & 6.3786 & 1.741605 \\
\hline .798885 & 3631.495 & .00475 & 4.2682 & 0793 & 0.9668 & 57.24991 & 79.83486 & 44.15787 & 16.28486 & & 10.36272 & 89.269 & 2.347332 & 5.7342 & 1.097526 \\
\hline 425516 & 7688.649 & 5976 & 547.1502 & 1603 & 9.4432 & 70266 & 29847 & 383 & 28.43224 & 5281 & 18.42985 & 17.367 & 2.074532 & .1029 & 1.282159 \\
\hline 005132 & 3951.004 & .9337 & 466 & 6255 & 2.0449 & 23.58461 & 89553 & 27.89536 & 14.30829 & 185005 & 9.474157 & 5.034 & 0.979152 & 6.8999 & 0.517297 \\
\hline 170204 & 33.049 & 7958 & 1.7179 & 841 & 150.5852 & 59.20152 & 44 & 14143 & 158 & 266 & 13.12192 & 81 & 0.532082 & 3.2803 & 1.244353 \\
\hline 34873 & 9.412 & 108 & 39 & & 46 & 63 & 143 & 809 & 708 & 688 & 2939 & 867 & 5949 & 5906 & .71037 \\
\hline 8145 & 880.48 & 07 & 58 & 775 & 60026 & & & & 041 & & 2.88959 & & & 76 & \\
\hline
\end{tabular}


Helictotrichon pratense

Helictotrichon pubescens

Heracleum sphondylium

Herminium monorchis

Herniaria glabra

Hieracium lactucella

Hieracium laevigatum

Hieracium sabaudum

ium sect. Hieraciu

Hieracium sect. Vulgata

Hieracium umbellatum

Hierochloe odorata

Hippophae rhamnoides

Holcus molls

Honckenya peploides

Hordeum murinun

Hordeum secalinum

Hordeum vulgare

Hottonia palustris

Humulus lupulus

Hydrocharis morsus-ranae

Hydrocotyle vulgaris

Hypericum dubiu

Hypericum elodes

Hypericum hirsutum

Hypericum humifusum

Hypericum pulchrum

Hypericum tetrapterun

Ilex aquifolium

Illecebrum verticillatum

Impatiens noli-tanger

Inula britannica

ula conyzae

Isolepis setacea

Jasione montana

Juncus acutiflorus

Jncus alpinoarticulatus s. atricapillus

Juncus ambiguus

Juncus balticus

Juncus bufonius

Juncus bufonius ag. (incl. J. ambiguus)

Juncus bulbosus

Juncus compressus

Juncus conglomeratus

Juncus conglomeratus $\mathrm{x}$ effusus

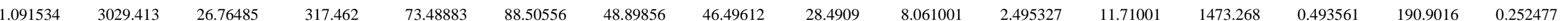

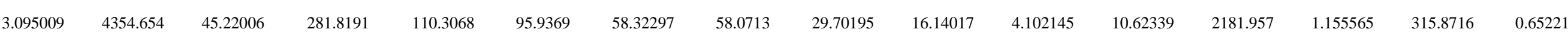

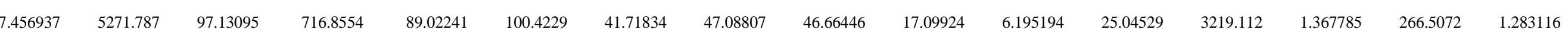

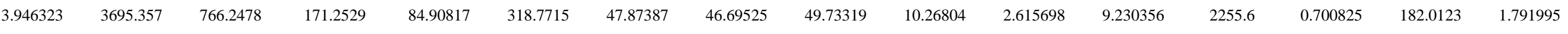

\begin{tabular}{llllllllllllllll}
4.001901 & 3613.434 & 79.26491 & 160.9731 & 100.7423 & 119.9266 & 57.26196 & 70.39705 & 27.77744 & 14.39736 & 3.478457 & 14.0291 & 2454.623 & 1.529511 & 349.0016 & 1.645745 \\
\hline
\end{tabular}

$\begin{array}{llllllllllllllll}6.76804 & 5630.673 & 57.01855 & 297.0405 & 68.54879 & 141.6549 & 46.78042 & 52.04226 & 26.71213 & 28.83961 & 5.27963 & 9.444033 & 5438.441 & 0.493493 & 274.826 & 1.485765\end{array}$

\begin{tabular}{llllllllllllllll}
7.839575 & 4027.157 & 40.11906 & 817.7162 & 79.81315 & 108.6211 & 55.61304 & 82.15546 & 46.07314 & 15.35424 & 11.63363 & 15.43587 & 2897.276 & 2.318185 & 294.3094 & 1.655888 \\
\hline
\end{tabular}

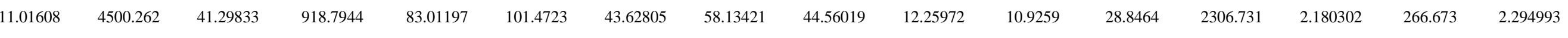

\begin{tabular}{llllllllllllllll}
9.254466 & 2586.844 & 43.43711 & 777.6828 & 52.92372 & 78.62141 & 39.68021 & 41.67237 & 30.89511 & 8.672185 & 7.238013 & 29.23509 & 1938.499 & 2.566917 & 358.6867 & 1.441586 \\
\hline
\end{tabular}

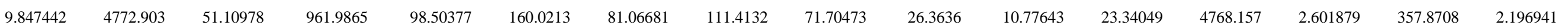

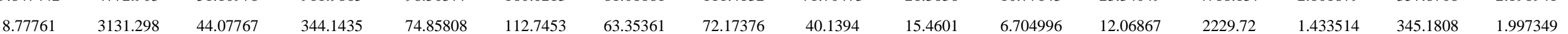

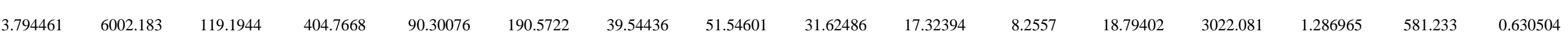

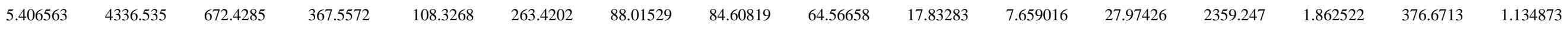

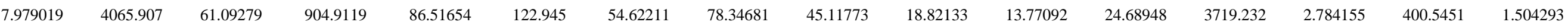

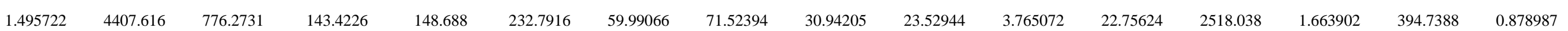

$\begin{array}{llllllllllllllll}3.072128 & 4709.359 & 87.99007 & 601.9611 & 174.4634 & 142.8315 & 31.16572 & 35.90859 & 39.02868 & 11.40764 & 3.131458 & 19.94845 & 2879.031 & 1.209668 & 512.3597 & 0.627237\end{array}$

$\begin{array}{llllllllllllllll}1.457322 & 3418.674 & 685.4669 & 379.559 & 89.06065 & 88.50506 & 20.24805 & 25.86949 & 22.27798 & 12.41613 & 2.859716 & 7.474381 & 2570.296 & 0.651998 & 246.7372 & 0.994209\end{array}$

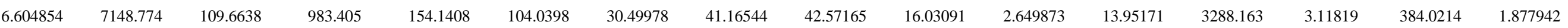

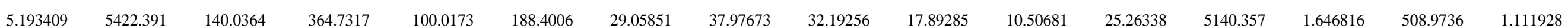

$\begin{array}{llllllllllllllll}4.838892 & 3985.063 & 84.08514 & 459.4112 & 74.99617 & 165.0345 & 43.0273 & 52.64998 & 50.6804 & 17.35281 & 9.671242 & 17.88709 & 3271.851 & 2.309405 & 268.1664 & 1.080973\end{array}$

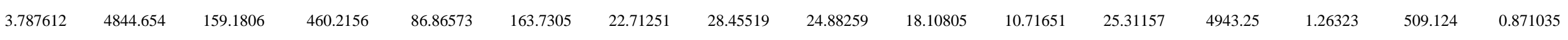

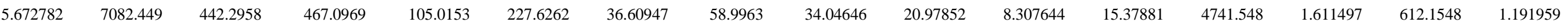

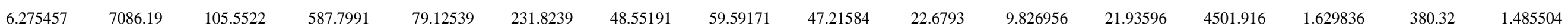

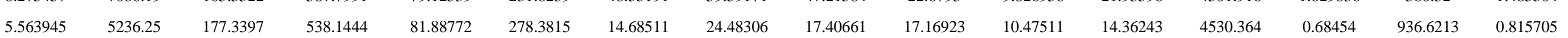

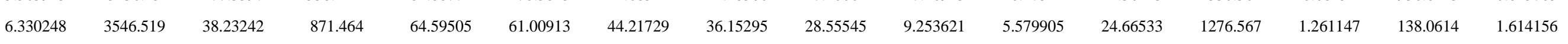

$\begin{array}{llllllllllllllll}6.966663 & 5405.69 & 121.719 & 765.8132 & 99.85275 & 142.395 & 66.46151 & 95.65997 & 66.60927 & 25.43699 & 7.951179 & 13.54839 & 4545.095 & 1.663333 & 365.3044 & 1.375896\end{array}$

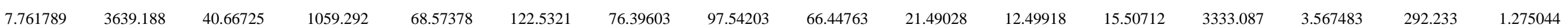

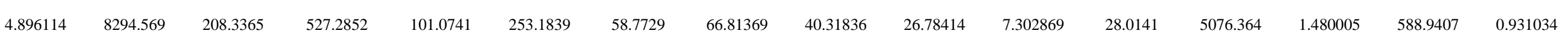

$\begin{array}{llllllllllllllll}5.134081 & 2144.962 & 26.39889 & 365.2651 & 50.9728 & 80.73927 & 38.99936 & 58.91824 & 30.53762 & 12.57604 & 8.596134 & 25.5057 & 1640.379 & 2.093482 & 287.8079 & 1.233919\end{array}$

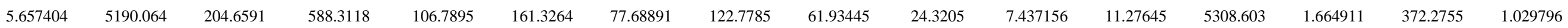

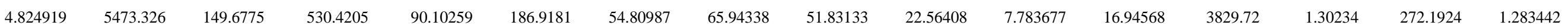

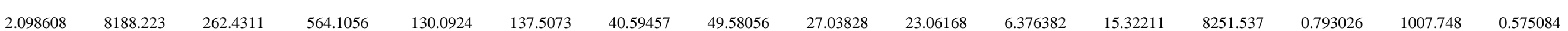

$\begin{array}{llllllllllllllll}7.150783 & 3560.688 & 57.20281 & 551.7196 & 83.29379 & 119.7682 & 55.95075 & 47.2369 & 43.47304 & 12.96727 & 6.809256 & 33.63574 & 2312.068 & 1.345291 & 312.1404 & 1.117921\end{array}$

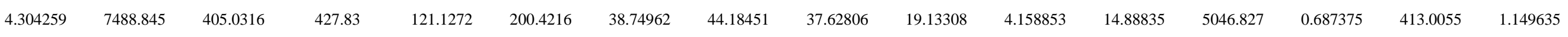

\begin{tabular}{llllllllllllllll}
5.940461 & 2666.711 & 25.39773 & 208.3357 & 60.98872 & 88.95469 & 59.05139 & 57.58729 & 31.79128 & 13.07043 & 3.305481 & 9.295957 & 1856.09 & 0.61087 & 290.3474 & 1.02506 \\
\hline
\end{tabular}

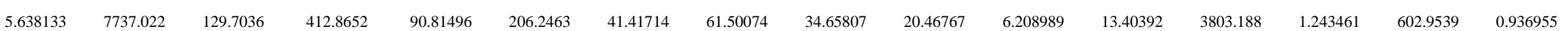

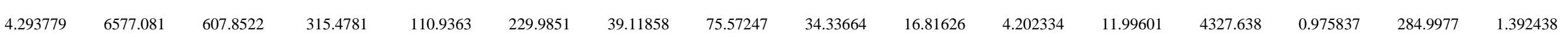

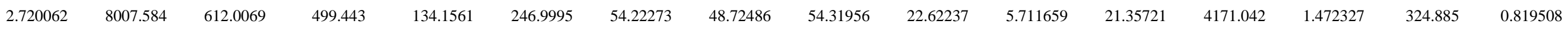

$\begin{array}{llllllllllllllll}4.475034 & 6775.37 & 513.2687 & 439.2212 & 125.9501 & 204.363 & 39.93625 & 52.73307 & 41.71939 & 24.3109 & 5.359728 & 16.34128 & 7476.276 & 1.087526 & 767.5236 & 1.466198\end{array}$

\begin{tabular}{llllllllllllllll}
6.259968 & 7452.676 & 630.2971 & 408.8947 & 110.1052 & 261.7425 & 38.38092 & 76.6249 & 29.52652 & 26.4772 & 3.968138 & 10.81735 & 4533.739 & 1.380364 & 339.8239 & 1.86501 \\
\hline
\end{tabular}

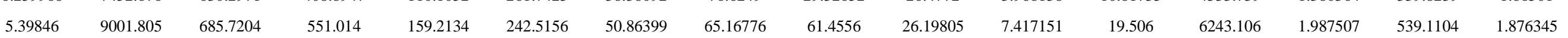

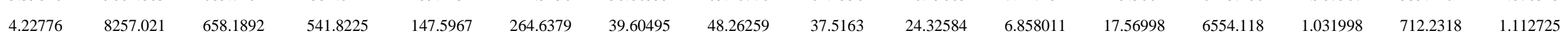

$\begin{array}{llllllllllllllll}10.2861 & 6038.53 & 232.5147 & 541.3087 & 91.21657 & 255.5274 & 22.28877 & 31.37268 & 22.24366 & 21.72629 & 8.002158 & 13.07331 & 4697.991 & 0.915542 & 558.5478 & 1.170682 \\ \end{array}$

$\begin{array}{llllllllllllllll}7.294968 & 8120.034 & 291.7409 & 445.7331 & 118.6154 & 234.7377 & 33.98773 & 43.46147 & 33.35013 & 26.52793 & 4.891505 & 25.14193 & 7735.687 & 0.958046 & 588.0513 & 1.767307\end{array}$

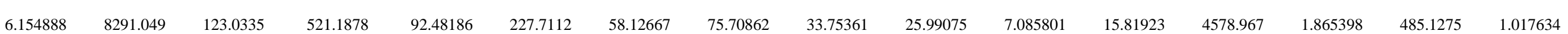

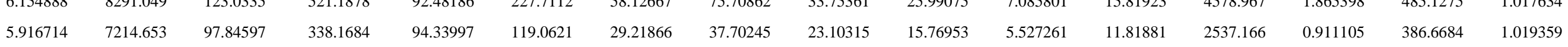




\begin{tabular}{|c|c|c|c|c|c|c|c|c|c|c|c|c|c|c|c|c|}
\hline Juncus effusus & 7.470487 & 9139.078 & 176.895 & 583.5115 & 142.8727 & 267.0276 & 75.44659 & 111.1645 & 54.20945 & 32.14857 & 11.56116 & 30.03024 & 5719.704 & 2.266715 & 744.1073 & 1.249572 \\
\hline Juncus filiformis & 4.979304 & 6599.751 & 112.7377 & 324.653 & 129.7548 & 165.0086 & 30.38831 & 34.16819 & 27.10532 & 16.97919 & 7.015331 & 10.7565 & 2780.757 & 1.346504 & 709.1015 & 2.099901 \\
\hline Juncus gerardi & 3.104848 & 6062.979 & 592.8754 & 394.6137 & 101.6581 & 159.1855 & 36.79192 & 31.39082 & 42.8686 & 14.91984 & 5.41709 & 12.4908 & 3838.805 & 1.016022 & 286.461 & 0.883093 \\
\hline Juncus inflexus & 5.187783 & 6450.096 & 393.4968 & 529.3931 & 111.3181 & 181.2673 & 43.47576 & 49.71624 & 45.88532 & 25.57155 & 6.271594 & 21.14241 & 6452.949 & 1.258847 & 699.0945 & 1.272897 \\
\hline Juncus maritimus & 5.016539 & 5438.283 & 603.6811 & 327.0564 & 136.2432 & 214.2266 & 43.23386 & 36.15204 & 37.15345 & 15.87405 & 5.758134 & 11.79502 & 3968.224 & 0.712525 & 229.3768 & 1.027423 \\
\hline Juncus squarrosus & 9.038535 & 3569.543 & 18.70813 & 833.5603 & 73.19743 & 128.1244 & 93.19604 & 132.694 & 51.87366 & 20.51339 & 6.003393 & 7.353987 & 4004.899 & 1.949355 & 351.8902 & 0.935258 \\
\hline Juncus subnodulosus & 5.909981 & 7794.014 & 177.3771 & 433.616 & 134.8714 & 191.4434 & 34.36189 & 51.56863 & 22.82618 & 23.13869 & 10.58067 & 29.74683 & 4495.971 & 1.817309 & 775.2599 & 1.061954 \\
\hline Juncus tenageia & 5.540809 & 8476.644 & 498.2091 & 726.4959 & 111.6411 & 199.1473 & 39.95434 & 56.28313 & 37.93689 & 13.86644 & 5.3147 & 11.3531 & 3318.955 & 0.482853 & 357.8685 & 1.179187 \\
\hline Juniperus communis & 6.95192 & 1522.354 & 26.81575 & 683.0512 & 46.35881 & 75.55717 & 57.51819 & 69.9541 & 45.98126 & 13.77533 & 10.20436 & 8.75893 & 2425.398 & 3.180419 & 444.7885 & 1.045901 \\
\hline Kickxia elatine & 3.554834 & 2688.516 & 69.57151 & 841.1914 & 121.8457 & 72.51546 & 26.85614 & 26.99085 & 27.62593 & 11.21146 & 2.655068 & 10.07523 & 1831.49 & 1.734152 & 209.8931 & 0.519458 \\
\hline Knautia arvensis & 2.207944 & 4122.064 & 48.46315 & 420.972 & 83.4299 & 88.05631 & 36.58042 & 39.32786 & 33.5642 & 14.31063 & 3.742253 & 11.78052 & 2678.646 & 0.613619 & 333.681 & 0.561369 \\
\hline Koeleria macrantha & 2.013727 & 2415.024 & 37.7449 & 179.0371 & 49.81423 & 92.05822 & 53.96141 & 49.44902 & 26.72789 & 12.27212 & 4.780498 & 9.867817 & 1603.388 & 1.351958 & 264.5166 & 1.138989 \\
\hline Koeleria pyramidata & 1.398409 & 3396.811 & 21.32034 & 236.0471 & 72.62137 & 152.0875 & 73.39281 & 43.09565 & 18.74985 & 9.814128 & 2.790594 & 9.319244 & 1492.188 & 0.506644 & 168.9963 & 0.293084 \\
\hline Lactuca serriola & 5.63192 & 4847.57 & 424.6203 & 880.0324 & 144.6121 & 136.1906 & 42.37939 & 52.3403 & 56.60292 & 19.92763 & 5.896254 & 28.7507 & 4434.576 & 2.123868 & 467.7684 & 0.820851 \\
\hline Lamiastrum galeobdolon & 3.707776 & 2921.618 & 37.84123 & 291.0602 & 53.97501 & 83.51432 & 36.12692 & 32.21153 & 24.29724 & 11.7454 & 6.990302 & 17.11188 & 1824.117 & 1.870426 & 144.285 & 0.873535 \\
\hline Lamium album & 6.463295 & 3778.619 & 61.29343 & 758.0534 & 88.66351 & 84.75974 & 32.66574 & 37.91658 & 22.9146 & 12.13081 & 7.171864 & 21.97749 & 2960.763 & 1.348668 & 341.1983 & 1.308373 \\
\hline Lamium amplexicaule & 2.420968 & 3753.447 & 111.1457 & 841.3964 & 133.607 & 102.7602 & 27.14397 & 33.58119 & 37.9773 & 10.69893 & 2.971606 & 14.39378 & 2027.091 & 1.952021 & 333.1247 & 0.696484 \\
\hline Lamium maculatum & 5.644373 & 3486.761 & 60.61795 & 743.2425 & 68.09663 & 79.64866 & 37.94314 & 34.67455 & 23.16555 & 10.85899 & 6.290793 & 18.42199 & 1981.858 & 1.012196 & 214.9984 & 1.22587 \\
\hline Lamium purpureum & 4.877318 & 4049.483 & 93.1368 & 721.5446 & 131.1238 & 99.59564 & 33.28929 & 39.63286 & 41.021 & 14.83049 & 4.312823 & 18.45947 & 3301.222 & 1.675394 & 480.0272 & 0.925886 \\
\hline Lapsana communis & 6.99007 & 3895.122 & 70.80427 & 683.6807 & 90.29847 & 98.50603 & 39.72248 & 47.13045 & 34.83387 & 16.13072 & 9.002515 & 23.95783 & 2792.68 & 1.994567 & 205.7451 & 1.53266 \\
\hline Lathyrus linifolius & 4.643766 & 2255.572 & 35.15436 & 685.6945 & 52.32888 & 79.3509 & 39.10209 & 49.08195 & 53.72488 & 9.353259 & 11.14504 & 15.24403 & 1723.337 & 3.631908 & 268.3327 & 0.817829 \\
\hline Lathyrus palustris & 3.713729 & 5161.691 & 126.177 & 429.165 & 100.2253 & 193.5648 & 33.19763 & 41.49973 & 24.41013 & 18.49799 & 8.890242 & 17.7498 & 3085.71 & 1.282742 & 642.6744 & 0.563706 \\
\hline Lathyrus pratensis & 3.340695 & 7498.688 & 145.298 & 417.3504 & 120.7026 & 129.7822 & 40.43345 & 48.96555 & 33.35402 & 28.88138 & 4.518084 & 11.42871 & 5529.414 & 0.683864 & 513.249 & 0.738875 \\
\hline Lathyrus tuberosus & 3.683364 & 4017.771 & 100.315 & 592.8439 & 109.1082 & 82.67175 & 27.85086 & 32.03568 & 35.6502 & 17.49418 & 4.911305 & 22.36415 & 2766.787 & 1.963928 & 351.0282 & 0.512135 \\
\hline Legousia speculum-veneris & 2.753713 & 2577.096 & 81.6735 & 507.0938 & 75.31121 & 75.03779 & 25.03161 & 25.9303 & 48.54855 & 11.91392 & 2.79412 & 9.270793 & 1930.635 & 2.898306 & 220.2074 & 0.419954 \\
\hline Lemna gibba & 4.194445 & 5085.307 & 225.279 & 370.0192 & 103.3284 & 190.7939 & 31.59003 & 38.87057 & 30.40236 & 21.32014 & 8.538701 & 26.08859 & 5396.738 & 1.703634 & 552.2312 & 1.177242 \\
\hline Lemna gibba + L. minor & 4.736669 & 7017.649 & 237.8423 & 426.3392 & 112.0319 & 186.0844 & 25.68738 & 37.87287 & 28.43809 & 21.61746 & 9.465296 & 25.90862 & 5444.085 & 1.461015 & 490.0492 & 1.036551 \\
\hline Lemna minor & 5.022608 & 6433.867 & 192.556 & 426.6297 & 108.1813 & 192.4191 & 30.38342 & 40.26645 & 31.23438 & 20.53896 & 10.63876 & 26.29391 & 5038.754 & 1.524033 & 520.3918 & 1.063884 \\
\hline Lemna trisulca & 4.100772 & 4907.91 & 203.0597 & 504.8499 & 90.28728 & 179.7243 & 23.86954 & 29.90415 & 28.20009 & 18.61057 & 9.963694 & 25.36219 & 4848.182 & 1.194357 & 493.0218 & 0.888352 \\
\hline Leontodon autumnalis & 4.613974 & 8789.06 & 703.6129 & 400.3382 & 177.6999 & 174.6458 & 48.46839 & 59.6027 & 34.22256 & 22.57392 & 5.589082 & 13.4588 & 5920.031 & 1.02317 & 636.5434 & 1.444021 \\
\hline Leontodon hispidus & 2.742936 & 3767.658 & 54.08086 & 450.1051 & 77.85208 & 83.56386 & 39.30979 & 39.7422 & 23.66486 & 11.81223 & 3.157392 & 10.5926 & 2833.295 & 0.590137 & 292.9902 & 0.606978 \\
\hline Leontodon saxatilis & 4.415389 & 7672.13 & 700.1959 & 265.3563 & 141.8301 & 257.2001 & 91.57188 & 90.53594 & 49.61812 & 28.93338 & 4.958722 & 13.31346 & 3582.897 & 0.994735 & 435.7967 & 1.508938 \\
\hline Lepidium draba & 2.143442 & 4490.972 & 639.5657 & 457.4199 & 159.3801 & 112.1223 & 41.68418 & 46.34566 & 41.44887 & 20.68068 & 4.178705 & 22.96958 & 3200.586 & 1.685588 & 632.7486 & 1.08265 \\
\hline Leucanthemum vulgare & 3.725566 & 4972.494 & 66.74266 & 396.5328 & 89.41841 & 105.2184 & 39.87735 & 45.63714 & 27.27695 & 18.55592 & 4.157442 & 12.05983 & 3794.245 & 0.751081 & 320.0048 & 1.146679 \\
\hline Leucojum aestivum & 3.103168 & 7457.311 & 136.3774 & 889.9877 & 100.023 & 175.2037 & 39.76911 & 52.71399 & 37.51268 & 23.25793 & 6.174384 & 22.04895 & 4036.285 & 0.946629 & 251.6859 & 0.780639 \\
\hline Leymus arenarius & 1.818335 & 3735.5 & 765.3797 & 266.7331 & 102.1025 & 148.844 & 67.26344 & 80.47984 & 42.31517 & 17.36973 & 3.862463 & 26.63236 & 2421.672 & 1.965931 & 403.6986 & 0.777472 \\
\hline Limonium vulgare & 2.380214 & 7781.911 & 494.0556 & 276.1221 & 160.0106 & 302.4707 & 42.21869 & 49.50925 & 57.56354 & 18.77309 & 5.105535 & 31.96144 & 2255.005 & 0.369965 & 303.4916 & 0.755996 \\
\hline Linum catharticum & 3.878286 & 6240.435 & 587.0236 & 418.9905 & 103.2462 & 263.841 & 70.01707 & 70.72672 & 45.28339 & 23.75261 & 5.124982 & 11.54926 & 4190.719 & 0.617244 & 304.4471 & 1.161684 \\
\hline Liparis loeselii & 4.430297 & 5265.216 & 405.7722 & 473.1057 & 77.95746 & 176.1307 & 24.80687 & 42.50966 & 19.28814 & 18.88959 & 8.444643 & 13.31328 & 5880.155 & 1.811051 & 890.2705 & 1.230772 \\
\hline Listera ovata & 5.568666 & 3382.879 & 45.97324 & 772.5587 & 58.04551 & 116.0539 & 54.056 & 48.38639 & 49.06623 & 18.97262 & 5.898802 & 26.96986 & 3152.698 & 1.661299 & 297.925 & 1.540388 \\
\hline Lithospermum officinale & 4.658246 & 3360.76 & 53.22439 & 527.1599 & 74.20224 & 104.6039 & 37.33011 & 41.30947 & 36.99342 & 12.59095 & 4.627431 & 25.02769 & 2109.229 & 1.815062 & 290.1766 & 0.806119 \\
\hline Littorella uniflora & 6.085464 & 6394.138 & 455.9441 & 540.733 & 99.41683 & 212.0277 & 14.91493 & 27.81687 & 15.58771 & 13.636 & 5.076863 & 14.48833 & 3607.114 & 0.772911 & 356.9436 & 1.288261 \\
\hline Lolium multiflorum & 4.957084 & 6367.763 & 124.4652 & 562.3399 & 123.9595 & 106.0373 & 33.72471 & 47.94168 & 47.51863 & 22.87441 & 5.569765 & 14.72938 & 4550.313 & 1.989333 & 405.2503 & 0.955478 \\
\hline Lolium perenne & 3.290379 & 5108.498 & 586.1911 & 514.8692 & 117.7627 & 105.1008 & 31.78021 & 39.68733 & 34.15808 & 16.76363 & 4.557054 & 11.0906 & 4060.371 & 1.046067 & 429.3898 & 1.050343 \\
\hline Lonicera periclymenum & 6.790628 & 3390.637 & 50.99499 & 709.1404 & 72.64165 & 127.8324 & 52.64448 & 75.39115 & 42.72565 & 17.19714 & 10.09711 & 27.37288 & 3700.911 & 2.600643 & 452.5245 & 1.524355 \\
\hline Lotus corniculatus v. & 4.72267 & 5098.765 & 672.941 & 306.3649 & 114.5173 & 158.9412 & 80.64047 & 79.82626 & 44.18865 & 22.04357 & 4.681857 & 12.13322 & 3274.274 & 912488 & 374.8091 & 416537 \\
\hline
\end{tabular}


Lotus glaber

Lotus pedunculatus

Luzula multiflora

Luzula multiflora s. congesta

Luzula multiflora s. multiflora

Luzula pilosa

Lychnis flos-cuculi

Lycopodiella inundata

copodium clavatum

Lycopus europaeus

Lysimachia nemorum

Lysimachia nummularia

Lysimachia vulgaris

Lythrum portula

Maianthemum bifolium

Malus sylvestris

Malva moschata

Malva neglect

Malva sylvestris

Matricaria discoidea

Matricaria recutita

Medicago arabic

Medicago falcat

Medicago falcata + M. sativ

Medicago lupulin

Medicago sativa

Melampyrum pratense

Melica uniflor

Melilotus altissimus

Melilotus officinalis

Mentha aquatica

Mentha arvensis

Mentha $x$ verticillata

Menyanthes trifoliat

Mercurialis annua

Mercurialis perennis

Mespilus germanica

um effusum

Milium vernale

Minuartia hybrida

Moehringia trinervia

ia caerule

Mycelis muralis

Myosotis arvensis

Myosotis discolor

\begin{tabular}{|c|c|c|c|c|c|c|c|c|c|c|c|c|c|c|c|}
\hline .273646 & 6263.182 & 605.7836 & 407.8761 & 128.9376 & 190.8156 & 39.70263 & 40.35634 & 39.31825 & 20.11142 & 5.108126 & 13.78449 & 3490.044 & 1.029173 & 316.0023 & 0.99615 \\
\hline 3.99955 & 6900.102 & 266.4831 & 426.1892 & 92.52302 & 195.1506 & 37.46767 & 46.83454 & 30.07977 & 22.62414 & 5.891852 & 13.87656 & 4521.341 & 1.03818 & 523.6701 & 0.751511 \\
\hline 316192 & 8480.981 & 110.5645 & 01.7588 & 100.4229 & 260.822 & 88.09427 & 123.7338 & 39.30011 & 36.92018 & 5.932788 & 11.14692 & 6170.041 & 2.029008 & 437.0524 & 1.2324 \\
\hline 23716 & 9720.499 & 54.3387 & 7.3269 & 84.97741 & 235.6402 & 65.76731 & 88.03092 & 33.90343 & 28.50601 & 6.163682 & 10.11075 & 5124.9 & 0.968387 & 390.9578 & 0.960 \\
\hline 09646 & 7213.21 & 61.33593 & 92.5612 & 85.54537 & 226.5702 & 76.32163 & 105.7403 & 54.21107 & 31.07746 & 9.055165 & 21.04736 & 5265.702 & 3.154346 & 368.4181 & 1.298668 \\
\hline 141929 & 2761.277 & 34.10768 & 377.138 & 57.99877 & 88.5577 & 32.70451 & 49.23242 & 23.90052 & 12.31315 & 8.98888 & 33.38464 & 1530.105 & 2.528513 & 213.6624 & 1.128873 \\
\hline 4.38003 & 4945.166 & 198.1793 & 424.9255 & 102.9139 & 150.3902 & 27.74578 & 34.69096 & 25.94426 & 19.1943 & 5.804264 & 15.90887 & 3481.822 & 0.95057 & 416.8688 & 0.711731 \\
\hline 103737 & 3904.425 & 14.5683 & 1.5161 & 63.94556 & 146.0637 & 63.86448 & 91.59326 & 34.93669 & 22.70882 & 5.403579 & 6.203025 & 3553.128 & 1.11225 & 201.1474 & 0.706447 \\
\hline 596321 & 1485.382 & 16.23086 & 1044.776 & 43.99681 & 77.01274 & 64.64093 & 79.04314 & 34.58046 & 14.02034 & 6.713837 & 5.81564 & 2005.54 & 2.578183 & 358.831 & 1.028712 \\
\hline 984279 & 7063.715 & 247.1073 & 34.8984 & 122.8037 & 243.8949 & 54.43971 & 77.46945 & 44.37018 & 23.81188 & 10.40117 & 28.23865 & 5721.273 & 1.45773 & 759.8173 & 1.559512 \\
\hline 515546 & 5342.207 & 60.39608 & 5.6654 & 70.90264 & 158.5851 & 47.47365 & 50.29177 & 50.75889 & 21.73992 & 5.992419 & 19.52593 & 3287.664 & 2.160489 & 87.4306 & 0.848564 \\
\hline 79715 & 7910.141 & 192.1818 & 5.7233 & 131.2486 & 191.0118 & 54.89305 & 68.96016 & 51.29381 & 27.27103 & 7.136389 & 25.30901 & 6351.793 & 1.087699 & 32.7975 & 1.180868 \\
\hline 88706 & 8103.267 & 169.3442 & 2.0059 & 141.099 & 185.434 & 50.13703 & 70.41957 & 34.14183 & 25.81245 & 8.874706 & 33.08458 & 5556.749 & 1.536396 & 01.4437 & 0.977 \\
\hline 5.88183 & 8391.78 & 128.6944 & 19.7133 & 104.7326 & 283.9193 & 73.07896 & 102.3336 & 53.4043 & 29.39596 & 10.23193 & 30.36668 & 4985.017 & 2.114173 & 60.4201 & 1.046009 \\
\hline .597232 & 7597.912 & 227.1653 & 6.0493 & 125.8046 & 239.5935 & 33.28876 & 49.47071 & 34.06619 & 20.88721 & 5.350342 & 18.44796 & 7504.556 & 0.832968 & 914.3384 & 0.912968 \\
\hline 209062 & 2577.569 & 31.25192 & 7.0378 & 55.64132 & 84.52741 & 34.91134 & 57.76743 & 27.73328 & 10.6775 & 8.735541 & 27.24694 & 1466.791 & 2.343741 & 185.8364 & 1.20459 \\
\hline 0.02302 & 4302.149 & 62.0828 & 20.7679 & 100.3127 & 128.9129 & 56.32181 & 70.8934 & 38.34202 & 14.57328 & 15.86337 & 31.17259 & 2854.822 & 3.184908 & 67.2068 & 2.744725 \\
\hline 028614 & 714.62 & 84.67851 & 3.368 & 18.3146 & 94.4336 & 28.80898 & 34.06379 & 41.71238 & 20.82332 & 5.387211 & 21.70759 & 4763.112 & 1.285462 & 06.0631 & 1.693255 \\
\hline 742658 & 659.949 & 126.5833 & 25.5198 & 91.59451 & 80.11326 & 26.53351 & 29.05053 & 34.09782 & 10.52833 & 3.290571 & 18.44639 & 2614.917 & 1.126505 & 378.319 & .046112 \\
\hline 983968 & 69.841 & .70505 & 9.7691 & 110.9702 & 102.5755 & 27.93504 & 32.89183 & 41.2056 & 11.91708 & 350732 & 20.64441 & 698.971 & 1.378201 & 13.2405 & 007797 \\
\hline 863097 & 73.681 & 7.0606 & 3412 & 144.3759 & 119.6931 & 31.81622 & 40.71254 & 42.13973 & 16.33048 & 462585 & 12.82905 & 3828.957 & 1.399574 & 34.1972 & . 059299 \\
\hline 387603 & 4268.484 & 515.7061 & 593.7238 & 139.2636 & 112.7153 & 29.84986 & 37.91271 & 44.67718 & 16.00092 & 3.896557 & 14.23632 & 3204.291 & 2.939666 & 402.9151 & .249621 \\
\hline .491795 & 3549.385 & 658.5063 & 83.2397 & 109.0868 & 106.5486 & 28.0526 & 30.42553 & 19.83442 & 11.0949 & 631481 & 10.50004 & 2457.325 & 1.418158 & 32.6632 & 0.654353 \\
\hline .401123 & 3742.169 & 57.43753 & 6.3073 & 86.74139 & 74.99522 & 33.38972 & 37.20679 & 27.40901 & 11.14935 & 3.232697 & 9.179874 & 2218.287 & .2344 & 95.973 & .399575 \\
\hline . 401311 & 2815.756 & 42.14964 & 8.5343 & 80.91442 & 71.03801 & 37.30476 & 35.85994 & 23.21272 & 10.30785 & 4.637336 & 9.277737 & 74.865 & 0.904851 & 918 & 32463 \\
\hline 96476 & 24.045 & 4.6389 & 4.4207 & 113.0972 & 100.5684 & 39.592 & 41.62895 & 25.04934 & 16.2611 & 4.526748 & 10.77578 & 774.106 & 0.933244 & 7.8613 & 0.557319 \\
\hline .667467 & 3614.506 & 85.98748 & 535.807 & 101.7482 & 82.02814 & 34.53107 & 35.68346 & 30.44784 & 11.96691 & 5.094659 & 12.27335 & 2759.619 & 0.592104 & 299.7721 & 0.786359 \\
\hline 8.556671 & 2523.096 & 40.23113 & 688.5711 & 65.93721 & 96.29005 & 62.78284 & 81.63229 & 39.19144 & 15.13426 & 11.08954 & 20.7152 & 2274.63 & 2.617583 & 299.0446 & .371479 \\
\hline 3.44487 & 2295.746 & 35.64643 & 265.3352 & 43.02921 & 60.66927 & 24.20388 & 20.0815 & 24.07202 & 8.189604 & 4.467861 & 15.72982 & 980.8821 & 1.158001 & 134.4829 & 0.838429 \\
\hline 3.166297 & 3058.267 & 609.8939 & 45.9939 & 84.68768 & 81.63084 & 42.82612 & 44.63861 & 35.94872 & 14.23885 & 5.799097 & 16.71445 & 2642.934 & 0.455133 & 27.4402 & 148 \\
\hline .275214 & 4577.785 & 245.0591 & 455.0477 & 127.6937 & 129.6151 & 44.68365 & 48.17314 & 45.49385 & 15.95494 & 2.714688 & 16.74048 & 2914.093 & 1.685167 & 21.0582 & 0.760657 \\
\hline 4.36322 & 6838.036 & 368.4699 & 541.6731 & 119.8794 & 212.6265 & 40.96588 & 61.73006 & 40.77188 & 26.6966 & 9.037678 & 30.39275 & 7624.364 & 1.388638 & 58.4317 & 1.189065 \\
\hline 4.63534 & 8292.851 & 276.2566 & 439.2014 & 136.7754 & 183.4564 & 54.47883 & 70.89006 & 55.53832 & 37.79247 & 8.651061 & 18.13579 & 6846.211 & 1.999852 & 531.3827 & 1.158954 \\
\hline .317549 & 3985.44 & 103.8085 & 7.2771 & 86.54743 & 140.9437 & 29.9902 & 38.5492 & 37.925 & 24.26625 & 5.32146 & 25.17075 & 7022.171 & 0.85189 & 567.4437 & .953023 \\
\hline 350155 & 5881.824 & 151.2613 & 2.0959 & 121.7604 & 164.3598 & 27.34426 & 44.75732 & 25.13958 & 20.89955 & 9.2857 & 29.66104 & 3762.047 & 1.957343 & 73.6277 & .812837 \\
\hline .012722 & 37.337 & .69963 & 4.4297 & 95.79596 & 77.81483 & 30.78025 & 34.55609 & 46.04812 & 13.5827 & 3.414302 & 18.12782 & 138.353 & 2.272752 & 64.7423 & .933552 \\
\hline 3.37096 & 77.298 & .99359 & .9638 & 8.48904 & 76.24516 & 33.72159 & 1.62737 & 0778 & 12.52299 & 4.445903 & 16.03365 & 15.394 & 0.978373 & 39.0413 & .87 \\
\hline 322612 & 22.728 & 68 & 58 & 1.57659 & .05202 & 43 & 79 & & 9.872061 & 9.740273 & 218 & 36 & 2.699762 & .5727 & .380763 \\
\hline 417634 & 31.216 & 36.42469 & 2892 & .50434 & 76519 & 32.664 & 37.26307 & 9534 & 11.25692 & 15 & 17.78251 & 1494.518 & 2.831562 & 1389 & 1.266065 \\
\hline 100435 & 92.772 & 40.2162 & . 6768 & 72.84884 & 1.97395 & 59.49359 & 48.60709 & 39.19775 & 10.55802 & 28339 & 10.06639 & 1673.439 & 1.935621 & 3.8818 & 0.75 \\
\hline .005515 & 77.468 & 39.78469 & . 1678 & 0.397 & 99.74566 & 321 & 3.43865 & 54.36067 & 11.54047 & 075011 & 9.703475 & 4.853 & 0.578829 & 9.8995 & .58194 \\
\hline .398678 & 33.173 & 21098 & 4.485 & 69.23863 & 3.6245 & 42.05499 & .74971 & 36.19434 & 16.66674 & 7.560212 & 27.52878 & 38.548 & 1.869243 & 0.6791 & 1.644053 \\
\hline 9.40983 & 3.313 & 63775 & 1296 & 6976 & 6.0335 & 11 & 4.0498 & 8139 & 33.32554 & .08967 & 12.4246 & 07.799 & 3.700715 & 1.1416 & .35138 \\
\hline 023043 & 5.634 & 32765 & 298 & 99823 & 163 & 42.09197 & .53369 & 31.99658 & 6271 & 15 & 20.49295 & 33.869 & 1.194773 & 8.3809 & 94117 \\
\hline 524088 & 6.344 & 0746 & & 44 & 31 & 24 & 10378 & & 31 & 14 & 643 & 296 & 761 & 477.2852 & 88 \\
\hline 60791 & 54.789 & 3441 & 565 & 397 & 1357 & 524 & 11.349 & 22984 & 1.73179 & & 302 & & $16-1$ & 533.246 & \\
\hline
\end{tabular}


Myosotis laxa + M. scorpioides Myosotis laxa s. cespitosa Myosotis ramosissin Myosotis scorpioides Myosotis stricta Myosotis sylvatica Myosurus minimus Myrica gale

Nardus stricta

Narthecium ossifragum Nuphar lutea

Nymphaea alba

Odontites vernus

Odontites vernus s. serotinu

Oenanthe aquatica

Oenanthe fistulos

Oenanthe lachenalii

Oenothera bienn

Oenothera erythrosepala

Oenothera parviflora

Ononis repens

Ononis repens s. repens

Ononis repens s. spinosa

Ophioglossum vulgatum

Orchis militaris

Orchis morio

Origanum vulgare

Ornithogalum umbellatu

Ornithopus perpusillus

Orobanche caryophyllace

Orobanche picridis

Osmunda regalis

Oxalis acetosella

Oxycoccus palustris

Papaver argemone

Papaver dubium

Papaver rhoeas

Parapholis strigosa

Paris quadrifolia

Parnassia palustris

Pastinaca sativa

Pedicularis palustris

Pedicularis sylvatica

Persicaria amphibia

Persicaria bistorta

\begin{tabular}{|c|c|c|c|c|c|c|c|c|c|c|c|c|c|c|c|}
\hline 829819 & 6791.435 & 344.0545 & 503.3958 & 115.374 & 243.7792 & 46.4059 & 67.3213 & 38.391 & 26.75486 & 9.146153 & 28.40074 & 6898.892 & 1.311112 & 624.9199 & 1.221376 \\
\hline 087841 & 6792.909 & 365.4267 & 438.7736 & 114.6161 & 209.9593 & 42.35611 & 60.57347 & 44.00897 & 26.29664 & 6.660749 & 24.7043 & 6261.643 & 1.196129 & 550.3874 & 1.15 \\
\hline 89071 & 594.501 & 56.9367 & 186.2868 & 56.92493 & 100.2494 & 61.09172 & 4.41335 & 31.03293 & 12.12452 & 4.856982 & 15.18586 & 1606.453 & 1.566231 & 268.4751 & 0.792217 \\
\hline 09904 & 35.868 & 190.8638 & 55.4575 & 00.2939 & 182.1225 & 30.08695 & 4.13814 & 32.27624 & 21.11573 & 6.267766 & 28.6789 & 6026.791 & 1.058134 & 471.4248 & 0.882781 \\
\hline 89565 & 054.33 & 14411 & 2.0427 & 68.42792 & 106.406 & 63.44021 & 1.60318 & 41.24023 & 3.24092 & 4.062939 & 16.98677 & 2058.757 & 3.411732 & 348.3447 & 1.501 \\
\hline 07037 & 2620.486 & 48145 & 8.3095 & 5.13092 & 55.00734 & 29.83856 & 23.37754 & 20.57718 & 9.192235 & 5.023813 & 16.26698 & 1385.106 & 0.871689 & 267.1451 & 0.742705 \\
\hline 94486 & 24.283 & 135.5345 & 14.6529 & 30.8729 & 111.8895 & 30.05884 & 35.57155 & 53.41206 & 17.65832 & 4.395863 & 10.3264 & 4346.759 & 1.064606 & 438.659 & 0.578238 \\
\hline 107333 & 673.357 & 139.685 & 445.6634 & 91.82394 & 316.6635 & 67.44289 & 98.19349 & 41.36408 & 31.98927 & 9.181594 & 19.91676 & 5102.597 & 2.337512 & 859.7954 & 1.044292 \\
\hline 55308 & 4510.646 & 21.07087 & 959.7318 & 90.63048 & 162.6238 & 97.80914 & 129.626 & 37.0178 & 34.89078 & 8.52647 & 7.776709 & 6274.822 & 1.056563 & 407.03 & 1.153672 \\
\hline 54783 & 3525.793 & 20.36792 & 84.0224 & 57.26137 & 173.5176 & 71.57388 & 107.5417 & 35.53843 & 23.62326 & 9.705506 & 6.292504 & 4056.81 & 2.742625 & 338.6447 & 0.661217 \\
\hline 61341 & 5247.198 & 154.2484 & 9.4774 & 82.81307 & 172.3937 & 23.22756 & 34.57458 & 23.33719 & 18.95834 & 10.55466 & 23.28256 & 5104.076 & 1.364818 & 35.5809 & 0.904 \\
\hline 81351 & 5664.907 & 9.2815 & 5.3959 & 103.887 & 144.3111 & 27.85445 & 41.34956 & 28.65503 & 20.25846 & 9.110887 & 40.85259 & 5312.509 & 1.395171 & 553.5522 & 1.132679 \\
\hline 68011 & 841.209 & 9.3643 & 7.7048 & 146.8541 & 233.5758 & 55.22235 & 53.76132 & 46.76416 & 26.39963 & 6.401642 & 13.87735 & 3460.373 & 0.834588 & 45.7166 & 0.851534 \\
\hline 76518 & 635.679 & 6.7406 & 0.3933 & 119.5469 & 278.7429 & 36.96498 & 41.01268 & 42.13071 & 21.19224 & 6.574519 & 13.71675 & 4652.053 & 1.067789 & 459.5635 & 1.027906 \\
\hline 634874 & 7074.441 & 7..3381 & 404.5639 & 112.5424 & 211.0295 & 40.82826 & 54.33912 & 41.01714 & 23.98089 & 11.14981 & 25.89051 & 6154.589 & 1.574102 & 479.2662 & 1.275909 \\
\hline 357996 & 4302.111 & 2.4001 & 0.8739 & 74.12076 & 202.5195 & 30.98302 & 39.50051 & 35.5601 & 20.59331 & 10.11803 & 23.65418 & 5700.791 & 1.01217 & 533.4625 & 0.740044 \\
\hline 697328 & 063.47 & 6.7125 & 5.3369 & 122.5005 & 124.2625 & 31.73267 & 31.25643 & 36.30099 & 12.18092 & 5.038128 & 19.49912 & 4441.297 & 1.044246 & 58.5312 & 0.919718 \\
\hline 474024 & 389.734 & 132.5367 & 364.1722 & 121.2473 & 143.5933 & 64.58129 & 63.44364 & 43.2793 & 17.509 & 5.455614 & 25.39144 & 2739.011 & 1.422793 & 435.3505 & 1.174932 \\
\hline 2.42426 & 749.627 & 0.7016 & 2.9172 & 139.0651 & 134.7897 & 43.9622 & 55.68345 & 45.25877 & 17.79234 & 158113 & 25.72039 & 015.142 & 2.227839 & 57.2767 & 0.934404 \\
\hline 899178 & 5273.03 & 769.2096 & 189.8678 & 149.663 & 234.0957 & 84.5914 & 106.9586 & 58.78482 & 13.96899 & 882888 & 24.81652 & 897.588 & 1.693998 & 61.3612 & .182261 \\
\hline 278516 & 6168.561 & .86131 & 183.644 & 116.7448 & 166.5186 & 88.41909 & 100.6681 & 42.42047 & 23.91619 & 4.643514 & 14.82695 & 2633.064 & 0.729668 & 391.5248 & 1.518866 \\
\hline .957986 & 3172.222 & .80607 & 216.509 & 77.40745 & 117.3272 & 60.82717 & 53.15079 & 28.60981 & 14.02484 & 526835 & 11.52499 & 877.305 & 1.074446 & 3.5794 & 0.801567 \\
\hline .904893 & 4351.548 & 701.6842 & 319.5127 & 108.745 & 122.5426 & 45.64644 & 45.84035 & 27.25696 & 12.98195 & 615823 & 11.61096 & 661.063 & 0.849844 & 30.6224 & .522254 \\
\hline 776046 & 7637.044 & 521.3415 & 405.4721 & 88.75041 & 168.222 & 49.37173 & 46.62102 & 40.59777 & 21.34163 & 5.192136 & 10.71653 & 833.628 & 1.040659 & 0.5691 & 0.947658 \\
\hline .421359 & 3819.438 & 43.11038 & 4.3385 & 65.94819 & 72.05631 & 41.36147 & 34.38192 & 41.6389 & .232593 & 75 & 30.56558 & 31 & 0.486205 & 4.4032 & .248949 \\
\hline .711001 & 7861.865 & 13.5794 & 308.5732 & 97.73171 & 161.9375 & 76.24471 & 106.1801 & 34.66173 & 37.86072 & 7.047008 & 8.995295 & 33.501 & 0.65209 & 78 & 1.097247 \\
\hline 309733 & 3500.44 & 81367 & 80.3936 & 71.86758 & 81.68717 & 44.2379 & 36.39673 & 37.08197 & 8.584432 & 4.003556 & 19.00993 & 2134.403 & 0.561201 & 285.9071 & 0.500606 \\
\hline 367178 & 3767.485 & 46.08565 & 1104.829 & 71.64591 & 74.53801 & 39.36737 & 41.99309 & 19.39278 & 11.6216 & 7.162136 & 26.96569 & 2255.956 & 1.656368 & 332.7979 & 1.279825 \\
\hline .820766 & 3308.865 & 34.53712 & 244.8783 & 85.68387 & 110.6579 & 38.67326 & 52.4085 & 27.07669 & 14.78158 & 3.487801 & 10.15558 & 2347.861 & 1.925791 & 330.8427 & 0.914476 \\
\hline 1.679685 & 3076.941 & 32.62907 & 163.8561 & 87.58108 & 99.34516 & 58.5296 & 47.03318 & 25.23213 & 14.94249 & 3.166535 & 9.402827 & 1968.102 & 1.364286 & 02.7693 & 0.755627 \\
\hline 2.39967 & 1875.722 & 47.00407 & 11.8831 & 40.98084 & 67.32435 & 39.35863 & 39.14702 & 33.30378 & 14.89223 & 5.595398 & 7.662505 & 1760.27 & 1.882348 & 264.5531 & 0.72601 \\
\hline .345757 & 5463.151 & 125.6335 & 31.4777 & 85.94017 & 335.4667 & 94.71995 & 150.5139 & 73.5755 & 27.757 & 9.181148 & 28.25005 & 3905.052 & 2.759458 & 759.6512 & 1.236634 \\
\hline 4.64447 & 3372.31 & 39.22079 & 258.0202 & 68.64073 & 101.2912 & 38.42736 & 55.84869 & 27.11164 & 14.45296 & 9.343285 & 22.69182 & 1857.738 & 2.717255 & 170.5615 & 1.273723 \\
\hline 423537 & 3724.537 & 26.5311 & 5.7891 & 72.21281 & 296.2158 & 84.4523 & 135.3375 & 35.78131 & 27.48675 & 9.743848 & 6.949633 & 217.357 & 2.338452 & 616.571 & 0.599321 \\
\hline 247852 & 3238.786 & .69486 & 5.3993 & 114.0078 & 87.20505 & 28.39826 & 34.21753 & 39.82266 & 10.08363 & 2.672115 & 9.645838 & 335.675 & 2.827805 & 291.1578 & 0.857545 \\
\hline 94542 & 3693.857 & 52537 & 2.7616 & 107.2246 & 93.29512 & 29.70258 & 35.3778 & 40.18073 & 12.08766 & 47483 & 13.13111 & 10.668 & 3.143776 & 398.4469 & 0.863163 \\
\hline .396675 & 3311.606 & 05736 & 1.4325 & 109.6175 & 96.27743 & 27.19323 & 0.62359 & 40.4 & 13.62315 & 3.448966 & 13.90485 & 11.205 & 2.973405 & 370.8522 & 0.521627 \\
\hline 1.86742 & 9.635 & 5.9816 & 159.6646 & 509 & 6.045 & 39.92663 & 2.67816 & 33.93654 & 20.64772 & 7067 & 13.12322 & 21 & 0.561156 & 328.357 & .774841 \\
\hline 1.816491 & 7.728 & 6.1088 & 1.6365 & 77213 & 502 & 36.38648 & 9.16891 & 27.42961 & 3.26874 & 76254 & 11.18802 & 70.028 & 1.052163 & 52.16 & 1.921449 \\
\hline 1.923671 & 2592.785 & 40.24769 & .6107 & 00416 & .23654 & 35.48623 & 5.48027 & 23.48643 & 0.94894 & 522604 & 15.24608 & 14.318 & 0.824972 & 1.8211 & .672064 \\
\hline 495584 & 5573.356 & 510.917 & .5291 & 87.23884 & 1.8114 & 39.48882 & 45.91119 & 32.62341 & 21.21299 & 5.523126 & 11.48294 & 74.301 & 0.979088 & 9.8553 & . 496881 \\
\hline 36769 & 27.147 & 75179 & 8.8108 & 104.2091 & 04145 & 38.38882 & .03432 & 40.82712 & 15.51791 & 023 & 13.30075 & 41.635 & 1.03635 & 1.1661 & 0.596814 \\
\hline 716456 & 5325.296 & 321.7533 & 406.613 & 113.657 & 9.1468 & 31.00583 & 45.65165 & 30.99922 & 22.21573 & 44993 & 13.38244 & 11.102 & 1.541319 & 0.6009 & 0.752667 \\
\hline 82996 & 33.674 & 71048 & .4577 & 63.59271 & 172.7516 & 59.29987 & 76.6954 & 31.62734 & 2.12144 & 26404 & 77512 & 917 & 0.768313 & 0.4894 & 0.778644 \\
\hline 502478 & 8.919 & 005 & 14 & 599 & 114 & 16 & & 15 & 756 & 53 & 305 & .078 & 1.035125 & 2672 & 0.870422 \\
\hline 48022 & 27.399 & 74 & 58 & 103 & 5.1385 & 00612 & 152 & 55 & & 17 & 25.9224 & & & 96 & \\
\hline
\end{tabular}


Persicaria hydropiper

Persicaria lapathifolia

Persicaria lapathifolia s. lapathifolia

Persicaria lapathifolia s. pallida

Persicaria maculosa

Persicaria minor

Persicaria mitis

Petasites hybridus

um carvifolia

Peucedanum palustre

Phalaris arundinacea

Phleum arenarium

Phleum pratense

Phleum pratense s. pratense

Phleum pratense s. serotinum

Phragmites australis

Phyteuma spicatum s. nigrum

Picris hieracioides

Pimpinella major

Pimpinella saxifraga

Pinguicula vulgaris

Plantago coronop

Plantago lanceolat

Plantago majo

Plantago major s. intermedia

Plantago major s. major

Plantago maritim

Plantago media

Platanthera bifolia

Poa angustifolia

Poa annua

Poa compressa

Poa nemoralis

Poa palustris

Poa pratensis + P. angustifoli

Poa trivialis

Polygala comosa

Polygala serpyllifolia

Polygala vulgaris

Polygonatum multiflorum

Polygonatum odoratum

Polygonum aviculare

Polypodium vulgare

Populus nigra

Populus tremula

\begin{tabular}{|c|c|c|c|c|c|c|c|c|c|c|c|c|c|c|c|}
\hline 881274 & 8708.967 & 255.4248 & 497.5667 & 145.5424 & 301.329 & 49.89059 & 75.06853 & 43.5622 & 37.96657 & 8.847655 & 23.32817 & 7128.058 & 1.601286 & 514.766 & 70 \\
\hline 761034 & 179.337 & 372.6196 & 577.43 & 140.4842 & 202.7975 & 51.55033 & 65.25027 & 75.5328 & 32.3439 & 6.846348 & 16.67352 & 7632.7 & 2.525423 & 424.8397 & 1.353 \\
\hline 607799 & 8830.82 & 702.6288 & 578.406 & 159.7905 & 213.7708 & 41.28432 & 56.85597 & 78.12685 & 36.54375 & 5.010904 & 20.85185 & 8902.447 & 1.789891 & 359.4036 & 1.170102 \\
\hline 16127 & 4933.024 & 153.9057 & 494.8165 & 160.9256 & 5.3573 & 48.16636 & 69.91085 & 62.77542 & 13.59856 & 2.961542 & 17.63183 & 3749.863 & 2.483703 & 433.2964 & 1.337264 \\
\hline 63796 & 49.814 & 314.5274 & 4.1746 & 155.1071 & 8.5567 & 53.92151 & 75.75427 & 66.80994 & 32.67724 & 6.79459 & 18.05412 & 6370.794 & 2.178839 & 368.5131 & 1.3575 \\
\hline 15105 & 637.529 & 479.8272 & 9.8145 & 1.1948 & 316.7309 & 64.31427 & 86.79686 & 53.21829 & 32.88624 & 8.3616 & 33.28096 & 8521.474 & 2.196128 & 818.7026 & 1.71917 \\
\hline 53177 & 627.295 & 249.3714 & 1.4619 & 63.2283 & 261.1656 & 68.10871 & 101.5006 & 58.2109 & 43.87557 & 11.0477 & 28.53697 & 9148.276 & 2.408612 & 733.7044 & 1.724387 \\
\hline 21773 & 693.749 & 190.0109 & 1.8813 & 18.2905 & 145.53 & 57.70932 & 64.51937 & 52.14319 & 21.36705 & 8.327467 & 21.31398 & 3976.136 & 1.557537 & 339.574 & 2.023071 \\
\hline 22715 & 3882.497 & 58.3192 & 70.5906 & 82.37443 & 65.98186 & 27.94343 & 29.16575 & 25.27079 & 10.10073 & 1.660327 & 5.906522 & 2645.112 & 0.671389 & 267.8172 & 0.437221 \\
\hline 01277 & 7231.912 & 174.4482 & 42.1271 & 105.1748 & 208.6864 & 46.1817 & 68.88766 & 31.06868 & 20.94142 & 8.792441 & 33.29051 & 3556.581 & 1.682298 & 718.9832 & 0.844915 \\
\hline .23037 & 8736.611 & 193.9638 & 1.6349 & 143.1419 & 213.783 & 57.76587 & 81.92184 & 48.67965 & 30.15776 & 8.197203 & 23.46989 & 7712.673 & 1.366598 & 612.5144 & 1.305368 \\
\hline 424363 & 2052.244 & 49.52812 & 5.8013 & 43.8442 & 86.16562 & 55.40671 & 50.27771 & 26.25037 & 10.59194 & 3.348277 & 9.817284 & 1281.987 & 1.598941 & 214.3336 & 0.69578 \\
\hline 087164 & 085.975 & 140.7657 & 3.7391 & 140.2797 & 126.075 & 37.02508 & 45.70293 & 36.03696 & 19.24095 & 5.069296 & 13.56118 & 4380.096 & 1.273789 & 53.4045 & 0.918881 \\
\hline 99191 & 054.725 & 90.81494 & 66.0482 & 112.6872 & 91.9043 & 31.20617 & 38.80521 & 27.24631 & 16.703 & 3.417579 & 10.10037 & 3738.823 & 0.735979 & 442.6726 & 0.632137 \\
\hline 572961 & 841.603 & 113.7233 & 253.1039 & 137.5455 & 107.8592 & 46.70212 & 54.75306 & 31.76248 & 16.88088 & 3.552595 & 10.06575 & 3077.593 & 1.30423 & 344.1104 & 0.706678 \\
\hline 649669 & 8352.489 & 2.0805 & 9.0666 & 140.4918 & 260.2474 & 61.31773 & 83.10764 & 50.1183 & 30.03864 & 12.26503 & 33.89231 & 7438.242 & 1.88123 & 857.8412 & 2.152015 \\
\hline 347262 & 774.492 & .52503 & 50.3512 & 13078 & 139.1461 & 73.50981 & 73.14324 & 47.9126 & 29.74739 & 5.90885 & 25.96526 & 4225.741 & 1.891418 & 182.371 & 0.964588 \\
\hline 323082 & 4283.436 & 51.09863 & 353.0537 & 10.2893 & 130.9675 & 57.18461 & 53.63562 & 27.73022 & 17.38282 & 5.815105 & 13.86423 & 2570.425 & 1.151713 & 394.6402 & 0.657543 \\
\hline 5.39761 & 06.34 & .41426 & 1.2007 & .50371 & 72.09556 & 39.62711 & 42.73405 & 44.99663 & 14.63646 & 4.025339 & 15.7268 & 2743.842 & 0.590865 & 20.7172 & 0.813198 \\
\hline 179093 & 32.947 & 41.76678 & 333.013 & 89.93025 & 98.78191 & 48.95718 & 51.60823 & 27.71275 & 15.20435 & 4.242854 & 12.52014 & 2674.222 & 0.811588 & 37.0903 & .039872 \\
\hline 606542 & 4640.311 & 78.88691 & 563.4885 & 66.28633 & 277.1957 & 40.50363 & 45.40417 & 23.99385 & 20.20836 & 4.520239 & 9.417776 & 4099.545 & 0.576245 & 57.7584 & .927768 \\
\hline 578376 & 777.744 & 622.334 & 4.3128 & 7.1845 & 299.1323 & 50.95764 & 55.22858 & 40.2259 & 24.8426 & 5.188294 & 19.45238 & 2820.523 & 1.356926 & 400.2635 & .805364 \\
\hline .474257 & 7768.271 & 124.2141 & 385.7367 & 122.6739 & 166.7908 & 56.2773 & 66.77707 & 34.50731 & 31.22709 & 6.203635 & 13.07495 & 6114.323 & 1.148738 & 3.9156 & 1.211994 \\
\hline .158424 & 7271.005 & 689.1098 & $536.18 \mathrm{C}$ & 141.8872 & 179.3782 & 39.76927 & 47.93841 & 49.83256 & 21.09777 & 176149 & 16.05282 & 4599.914 & 1.694901 & 0.5987 & 1.328069 \\
\hline 364899 & 8423.369 & 7.5016 & 5.58 & 0.3518 & 175.5188 & 42.82602 & 50.21431 & 46.81139 & 29.67384 & 6.349774 & 16.82544 & 6711.444 & 1.183891 & 8.1731 & . 111217 \\
\hline 613517 & 6920.662 & 597.7824 & 540.0782 & 135.0412 & 137.7532 & 42.04735 & 51.89083 & 43.83629 & 20.60843 & 5.03604 & 12.62262 & 4506.827 & 1.112712 & 16.1344 & 1.159394 \\
\hline 78653 & 6497.121 & 2.8139 & 302.0871 & 128.0874 & 203.5779 & 35.4864 & 34.93976 & 32.425 & 15.43949 & 5.359103 & 11.17076 & 2808.452 & 0.71074 & 4.1811 & 0.854728 \\
\hline 040913 & 4189.379 & 61.70095 & 347.1001 & 89.2053 & 92.99998 & 43.108 & 42.65183 & 21.36522 & 12.47068 & 3.1066 & 9.175729 & 2722.253 & 0.662531 & 06.2595 & 0.494922 \\
\hline 939515 & 7014.005 & 162.5642 & 572.3625 & 77.00686 & 182.4887 & 52.56058 & 71.80818 & 23.97006 & 32.56256 & 5.304953 & 9.931336 & 5595.824 & 1.023778 & 19.7526 & 0.854338 \\
\hline 289206 & 5079.718 & 76.5379 & 443.1614 & 124.5886 & 132.9906 & 44.08775 & 52.41792 & 41.04329 & 17.48955 & 5.432434 & 16.39329 & 3308.425 & 0.746809 & 400.2185 & 1.062683 \\
\hline 092602 & 7429.924 & 515.6447 & 598.3555 & 183.8809 & 153.6167 & 47.3437 & 60.62467 & 46.32957 & 20.297 & 5.774088 & 15.04167 & 4810.65 & 1.758199 & 597.8899 & 1.776923 \\
\hline 399139 & 5022.439 & 162.1094 & 244.5372 & 110.0315 & 186.6335 & 96.57969 & 84.31965 & 77.6273 & 17.96244 & 6.434719 & 27.99503 & 2860.81 & 0.477426 & 400.5695 & 1.15302 \\
\hline .597304 & 2985.185 & 36.86701 & 370.8698 & 60.58622 & 89.82511 & 35.33812 & 39.66927 & 30.17881 & 10.7646 & 5.937992 & 18.94405 & 1942.633 & 1.439212 & 152.6157 & 1.08072 \\
\hline 975689 & 7609.647 & 267.7173 & 601.4638 & 127.0049 & 230.5646 & 56.81468 & 71.8622 & 51.84716 & 28.27716 & 10.02518 & 29.39323 & 7011.207 & 1.585037 & 19.2426 & 1.174065 \\
\hline 781803 & 6385.691 & 9.3725 & 88.9369 & 154.216 & 168.1123 & 67.9662 & 75.53085 & 41.14223 & 25.28098 & 6.016298 & 15.74836 & 4937.118 & 1.454005 & 09.6029 & 1.397392 \\
\hline 994095 & 6925.697 & 89.59871 & 5.4472 & 6.7309 & 135.4414 & 47.26968 & 3.32637 & 45.45314 & 20.17498 & 7.315317 & 17.1559 & 4488.292 & 2.068325 & 6.8775 & 1.822869 \\
\hline 676578 & 8190.457 & 64 & 3.64 & 993 & 165.5375 & 50.58338 & .53929 & 7.3665 & 26.9626 & 31867 & 24.02051 & 5064.763 & 1.484894 & 9.9265 & 1.442905 \\
\hline 670577 & 4.597 & 36.93018 & 552.8898 & 19 & 72.62224 & 46 & 327 & 20.64946 & 6.299628 & 86 & 12.31293 & 81 & 0.476408 & 453 & 0.533125 \\
\hline 78315 & 8.837 & 03685 & 93.0368 & 66282 & 8.0985 & 78893 & 117 & 37.42595 & 33.04016 & 51 & 7.87677 & 6139.062 & 0.821772 & 1.3805 & 0.893314 \\
\hline .909415 & 3927.743 & 7.9159 & 2.4795 & 3628 & 5.2507 & 74.50254 & 76.28256 & 47.14218 & 21.45061 & 64071 & 10.6617 & 2428.94 & 0.891071 & 0.1355 & 1.65397 \\
\hline .367148 & 2553.461 & 31.08538 & 396.2712 & 08171 & 88.21047 & 36.45821 & 47.10701 & 29.45301 & 10.75472 & 8.739981 & 25.65766 & 1605.575 & 2.440213 & 172.9964 & 1.239556 \\
\hline 03252 & 32.503 & 5692 & 480.211 & 68034 & 9712 & 00407 & 3.63304 & 28.18955 & 11.38218 & 813296 & 24.95229 & 1922.993 & 1.301493 & 323.006 & 1.507969 \\
\hline .779223 & 5472.729 & .3952 & 561.2008 & .9066 & 136.5558 & 34.57654 & .14262 & 124 & 18.77486 & 4.704999 & 13.62156 & 4445.902 & 2.62276 & 23.3283 & 1.365881 \\
\hline 17718 & 8.653 & 5092 & 821.56 & 63.02342 & .58916 & 2076 & .12196 & 34.61352 & 12.43002 & 89466 & 1753 & 29.206 & 3.666706 & 4.0701 & 2.098543 \\
\hline 893 & 6.539 & 107.2133 & 11 & 1035 & .3373 & & 332 & 913 & 027 & 64 & 3998 & 674 & 1.303545 & 8.1237 & 1.938796 \\
\hline 56672 & .222 & 37 & 38 & & 0.232 & 87 & 13429 & & & 316 & 7.0862 & .765 & 776 & 74 & \\
\hline
\end{tabular}


Populus $x$ canescens

Potamogeton crispus

Potamogeton lucens

Potamogeton natans

Potamogeton pectinatus

Potamogeton polygonifolius

Potamogeton pusillu

Potentilla anglica

Potentilla argentea

Potentilla erecta

Potentilla palustris

Potentilla reptans

Potentilla sterilis

Potentilla verna

Primula elatior

Primula veris

Prunella vulgaris

Prunus avium

Prunus padus

Prunus spinosa

Pecinum

Puccinellia maritima

Pulicaria dysenterica

Pyrola minor

Pyrola rotundifolia

Pyrus communis

Quercus petraea

Radiola linoides

nunculus acris

Ranunculus aquatilis

Ranunculus arvensis

Ranunculus auricomus

Ranunculus bulbosus

unculus circinatus

Ranunculus ficaria s. bulbilifer

Ranunculus lingua

Ranunculus peltatus

Ranunculus repens

Ranunculus sceleratus

Raphanus raphanistrum

Reseda lutea

\begin{tabular}{|c|c|c|c|c|c|c|c|c|c|c|c|c|c|c|c|}
\hline .239335 & 4337.985 & 69.17009 & 949.6045 & 110.9398 & 163.3716 & 64.28501 & 67.30875 & 43.69678 & 23.37999 & 12.05841 & 32.69389 & 4051.486 & 1.926802 & 517.9433 & 334 \\
\hline 395674 & 4174.775 & 172.9359 & 262.6843 & 66.3515 & 197.3814 & 20.51406 & 25.7828 & 29.95348 & 16.14685 & 5.685788 & 20.06927 & 4577.791 & 1.144331 & 446.216 & 0.525095 \\
\hline .77153 & 4512.829 & 213.5884 & 255.9912 & 67.78253 & 184.428 & 22.88367 & 33.1259 & 25.158 & 14.92713 & 9.207709 & 21.45128 & 4505.414 & 1.022296 & 471.4289 & 0.715927 \\
\hline 801568 & 305.209 & 213.3497 & 1.2219 & 18.5866 & 180.3559 & 29.7658 & 39.81326 & 32.83368 & 19.06801 & 11.54902 & 22.91365 & 5057.992 & 1.527475 & 510.0661 & 1.08579 \\
\hline 67941 & 754.484 & 252.4582 & 81.7235 & 81.96426 & 170.6092 & 21.13186 & 5.98612 & 34.29516 & 8.98026 & 5.460908 & 15.4044 & 4732.826 & 1.023644 & 450.9135 & 0.751688 \\
\hline .81356 & 108.982 & 274.0055 & 1.1102 & 127.8726 & 231.7171 & 15.21282 & 8.7584 & 17.00497 & 25.94416 & 9.606583 & 17.90604 & 6996.28 & 0.609022 & 938.5649 & 1.118069 \\
\hline 38754 & 231.588 & 196.3119 & 339.0683 & 71.84342 & 173.7993 & 16.46971 & 19.18023 & 27.86165 & 16.8003 & 4.900763 & 11.68985 & 4545.93 & 0.931397 & 486.5986 & 0.601481 \\
\hline 69801 & 8207.903 & 238.4882 & 485.8456 & 99.94069 & 223.9869 & 60.5054 & 79.55111 & 44.99106 & 23.44864 & 7.870486 & 12.31748 & 5314.838 & 1.628719 & 440.1394 & 0.977731 \\
\hline 01779 & 396.697 & 667.8276 & 449.1271 & 113.2148 & 155.682 & 47.1127 & 42.82666 & 51.95101 & 19.88364 & 5.794162 & 14.47964 & 5285.016 & 1.048923 & 508.0021 & 1.263122 \\
\hline 74832 & 3010.505 & 26.06378 & 172.1398 & 79.69735 & 97.48652 & 41.33109 & 54.14327 & 27.78136 & 10.81987 & 3.161315 & 9.258436 & 1830.872 & 1.679094 & 269.5199 & 1.259874 \\
\hline 40479 & 6251.448 & 84.79182 & 669.4194 & 92.25729 & 233.718 & 71.15508 & 98.53853 & 35.4036 & 29.916 & 5.961056 & 11.02935 & 5777.981 & 1.474953 & 333.4225 & 1.164095 \\
\hline 86773 & 7126.269 & 159.7445 & 450.5946 & 121.512 & 180.0475 & 32.28027 & 49.93811 & 29.93814 & 22.85221 & 10.00362 & 28.55837 & 3648.264 & 1.928175 & 715.4344 & 0.774625 \\
\hline 73518 & 7809.709 & 190.1909 & 501.9044 & 162.1692 & 146.7399 & 57.48319 & 65.14842 & 49.95216 & 26.61017 & 4.820917 & 15.41291 & 5533.448 & 0.886798 & 632.7605 & 0.605627 \\
\hline 48283 & 2824.881 & 36.5722 & 699.3613 & 50.18585 & 55.34969 & 40.9601 & 33.1423 & 23.20722 & 10.28751 & 5.944406 & 14.24549 & 1496.794 & 1.448087 & 196.8251 & 1.157818 \\
\hline 567092 & 775.205 & 27.42183 & 165.0552 & . .09086 & 85.45043 & 42.52986 & 46.09902 & 28.96071 & 12.33701 & 4.30699 & 10.22757 & 1529.962 & 0.66421 & 223.1253 & 0.844688 \\
\hline 18612 & 407.316 & 44.12162 & 393.9035 & 58.49615 & 122.5356 & 50.55874 & 47.27798 & 43.69253 & 24.68218 & 3.66583 & 16.99257 & 3168.612 & 0.983771 & 136.8604 & 0.71248 \\
\hline 853643 & 4280.057 & 57.34984 & 900.5861 & 80.54711 & 81.72424 & 60.9171 & 51.77325 & 44.51038 & 14.54024 & 6.766278 & 30.59782 & 30.081 & 0.795384 & 350.1284 & 1.58378 \\
\hline 60507 & 156.868 & 378.974 & 424.6008 & 99.87509 & 180.6012 & 53.79357 & 60.96541 & 35.82852 & 24.9665 & 5.337998 & 13.40449 & 803.701 & 0.826391 & 409.51 & 1.267795 \\
\hline 646817 & 606.122 & 38.73992 & 452.56 & 53.00856 & 85.30346 & 38.66795 & 40.35878 & 29.76261 & 11.21015 & 7.772391 & 20.88023 & 774.561 & 2.775633 & 216.5443 & 1.075055 \\
\hline 741312 & 399.614 & 45.40222 & 434.358 & 65.66959 & 126.366 & 40.12736 & 51.18927 & 44.81935 & 15.37576 & 7.41741 & 19.74419 & 2738.263 & 1.530462 & 39.6239 & .061261 \\
\hline 166444 & 3258.748 & 59.59539 & 958.9323 & 69.96952 & 90.78148 & 36.78629 & 39.97354 & 37.04845 & 12.05752 & 7.377938 & 22.64582 & 648.793 & 2.314939 & 47.7931 & .748054 \\
\hline 54935 & 045.596 & 35.80602 & 499.3945 & 59.00225 & 87.99969 & 54.10096 & 75.25064 & 32.60352 & 13.65886 & 09303 & 24.95444 & 1694.085 & 1.702902 & 436.8008 & 1.133686 \\
\hline 598904 & 5596.847 & 642.0198 & 415.6636 & 86.1677 & 192.6803 & 25.15237 & 28.66881 & 29.39919 & 15.94299 & 5.412598 & 13.09755 & 4392.95 & 1.524591 & 413.5097 & 0.89328 \\
\hline 674002 & 5671.799 & 561.031 & 524.887 & 71.99597 & 183.344 & 24.39914 & 25.53519 & 33.849 & 22.50162 & 5.256253 & 17.38415 & 103.315 & 1.602469 & 22.2997 & .709391 \\
\hline 138377 & 7256.442 & 598.9502 & 459.7941 & 113.5607 & 201.3327 & 54.71123 & 46.73398 & 52.45487 & 18.45278 & .21876 & 16.67106 & 64.158 & 1.338419 & 59.0327 & .888973 \\
\hline .290674 & 4397.878 & 346.1274 & 642.8071 & 90.25355 & 249.2346 & 98.35804 & 127.907 & 60.97688 & 16.5584 & 9.974683 & 10.7589 & 3281.554 & 1.686064 & 358.6996 & .595046 \\
\hline .866422 & 4797.275 & 446.027 & 353.331 & 87.13033 & 216.239 & 74.69027 & 81.91122 & 46.26493 & 21.00233 & 5.87736 & 12.63656 & $\{24.406$ & 1.457082 & 293.048 & 1.250977 \\
\hline 10.0321 & 5317.362 & 60.32238 & 900.6508 & 118.4424 & 123.7593 & 68.18661 & 61.60929 & 38.97183 & 22.53783 & 13.29579 & 31.05863 & 3516.162 & 3.199138 & 445.4979 & 2.247287 \\
\hline 1.135671 & 1938.554 & 40.68773 & 519.291 & 64.76407 & 88.90781 & 48.65884 & 70.13922 & 34.81967 & 14.95407 & 9.760814 & 35.90756 & 1696.776 & 4.125824 & 463.0025 & 1.043073 \\
\hline .737833 & 3305.68 & 50.115 & 937.3913 & 79.45509 & 120.9629 & 67.05922 & 91.08571 & 51.46984 & 18.49964 & 11.89638 & 34.69302 & 3596.597 & 2.787006 & 506.0109 & 1.618282 \\
\hline 1.963377 & 6249.662 & 650.8414 & 274.8425 & 108.0469 & 282.2338 & 56.11162 & 60.15157 & 42.89531 & 14.08826 & 4.194542 & 9.932909 & 3550.057 & 0.662078 & 255.9909 & 1.309365 \\
\hline .677883 & 6378.436 & 101.2198 & 393.5177 & 102.1173 & 126.037 & 38.51346 & 46.70939 & 34.59407 & 22.76605 & 5.012823 & 13.83456 & 4407.164 & 0.838716 & 390.5592 & 0.959029 \\
\hline 714846 & 7006.016 & 314.835 & 674.821 & 108.0225 & 208.0204 & 33.78872 & 44.13084 & 37.88099 & 20.66551 & 6.392288 & 22.57565 & 5378.799 & 1.16342 & 534.1725 & 1.140055 \\
\hline 106157 & 3348.439 & 80.91843 & 815.6457 & 123.08 & 78.06776 & 21.18552 & 25.07842 & 46.95829 & 10.79347 & 3.081587 & 9.896207 & 1780.976 & 1.258431 & 218.9016 & 0.470867 \\
\hline 399654 & 8719.485 & 61.94191 & 667.1923 & 93.36625 & 108.8064 & 68.53819 & 65.55443 & 47.85109 & 27.5071 & 5.538896 & 26.70241 & 4008.55 & 1.585994 & 186.2535 & 0.848702 \\
\hline 365549 & 4845.927 & 82.71438 & 293.965 & 111.7747 & 4.4211 & 48.25817 & .84231 & 24.58784 & 15.44404 & 2.715513 & 10.41806 & 938.14 & 0.990595 & 1.5872 & 0.820708 \\
\hline 45143 & 5401.906 & 7.5742 & 8.3561 & 2319 & 187.5242 & 19.95794 & 383 & 26.08375 & 17.28748 & 6.019024 & 16.28339 & 573 & 0.950023 & 13.5442 & 0.617687 \\
\hline 398141 & 7.319 & 100 & 7.1608 & 119.3008 & 436 & 52.13979 & 955 & 28 & 712 & 33 & 171 & & 1.284896 & 2.8452 & 1.222525 \\
\hline 4.39397 & 6019.272 & 372.9256 & 6.3957 & 5.925 & 159.3888 & 35.25225 & 55.68924 & 60892 & 5564 & 5.785567 & 2769 & 60.885 & 1.154665 & 8.7943 & .821935 \\
\hline 324727 & 4469.146 & 145.358 & 13.3325 & 93.21311 & 137.3816 & 22.06755 & 348 & 18591 & 20.69809 & 26 & 28.36125 & 4319.986 & 1.049853 & 4752 & 0.692839 \\
\hline 648846 & 4460.851 & 186.0296 & 7917 & 85.28654 & 1.1214 & 33.48331 & 6.48582 & 38.64675 & 20.51074 & 5.044803 & 17.99867 & 7.629 & 1.095041 & .6756 & .701054 \\
\hline .563128 & 7690.959 & 4.2075 & .0557 & 8.8739 & 155.6109 & 46.96343 & .00616 & 3201 & 26.49834 & 369168 & 20.41536 & 86.596 & 1.279981 & .7515 & 1.094702 \\
\hline 849421 & 4979.176 & . 1036 & .0818 & 54 & 152.4326 & 1575 & .02308 & 91749 & 16.40714 & 27474 & 10.10009 & 3883.777 & 1.216744 & 4.4655 & 0.809457 \\
\hline 503435 & 7341.761 & .0769 & 525.2169 & 105.5635 & 2.959 & 40.47521 & 32983 & 45.56454 & 092 & 49657 & 20.98024 & 511.29 & 1.22685 & 4.9345 & 1.15611 \\
\hline 259 & .071 & 95.79519 & 03 & 101.4899 & 266 & 91 & & & 599 & 33 & 197 & .859 & 5766 & 4.8715 & 0.868745 \\
\hline & 8.416 & 64 & 06 & 99 & & 833 & 07 & & 374 & 25 & & & & 55 & \\
\hline
\end{tabular}


Reseda luteola

Rhamnus frangula

Rhinanthus alectorolophus

Rhinanthus angustifolius

Rhinanthus mino

Rhynchospora alba

Rhynchospora fusca

Ribes nigrum

Ribes rubrum

Ribes uva-crispa

Rorippa amphibia

Rorippa microphylla

Rrippa microphylla + R. nasturtium-aquaticun

Rorippa nasturtium-aquaticum

Rorippa palustris

Rorippa sylvestri

Rosa canina

Rosa pimpinellifolia

Rosa rubiginosa

\section{Rosa rugosa}

Rosa villosa

Rubus caesius

Rubus corylifolius

Rubus fruticosus ag.

Rubus gratus

Rubus plicatus

Rubus pyramidalis

Rumex acetosa

Rumex cripsus $\mathrm{x}$ obtusifoliu

Rumex hydrolapathun

Rumex maritimu

Rumex obtusifolius s. obtusifolius

Rumex palustris

Rumex sanguineus

Rumex thyrsiflorus

Sagina apetala

Sagina maritima

Sagina nodosa

Sagittaria sagittifolia

Salix alba

Salix aurita $+S$. cinerea

Salix aurita $x$ cinerea

\begin{tabular}{|c|c|c|c|c|c|c|c|c|c|c|c|c|c|c|c|}
\hline 248155 & 3794.718 & 125.1459 & 480.2561 & 154.6368 & 99.00299 & 42.72703 & 40.50789 & 46.5887 & 15.97868 & 3.304019 & 30.54191 & 2558.833 & 2.120947 & 512.6873 & 0.691037 \\
\hline 31726 & 3323.815 & 59.81068 & 578.5179 & 73.01669 & 146.8413 & 79.514 & 101.8475 & 56.7585 & 20.10193 & 9.274463 & 25.493 & 3716.493 & 2.426851 & 478.7113 & 1.349789 \\
\hline 574454 & 3274.779 & 45.96719 & 480.4742 & 60.35948 & 57.55878 & 63.0509 & 40.28521 & 36.63582 & 8.324258 & 3.119097 & 7.927986 & 1343.585 & 0.447423 & 207.6887 & 0.611699 \\
\hline 09205 & 8854.355 & 3.2867 & 52.3742 & 43.9331 & 219.4393 & 60.19152 & 1.38925 & 30.07753 & 28.42732 & 7.235419 & 10.5131 & 7585.637 & 0.913195 & 728.6457 & 1.015652 \\
\hline 366494 & 8452.766 & 63.9978 & 75..324 & 125.6256 & 85.084 & 72.5905 & 75.21865 & 48.2957 & 27.73745 & 5.830134 & 11.21272 & 4553.172 & 0.682092 & 403.7874 & 1.102729 \\
\hline 79881 & 3097.723 & 10.23213 & 61.3556 & 61.18857 & 152.0516 & 63.7001 & 4.6441 & 31.68743 & 22.33782 & 10.44233 & 5.383307 & 3662.009 & 2.503708 & 230.8563 & 0.508988 \\
\hline 84133 & 415.439 & 12.03476 & 88.0216 & 4.43383 & 127.5429 & 59.1939 & 75.5494 & 30.73753 & 21.72862 & 6.784937 & 5.474439 & 3253.785 & 2.337103 & 214.6039 & 0.598245 \\
\hline 65064 & 4070.392 & 63.33313 & 303.3114 & 73.08802 & 156.3365 & 46.9507 & 57.45084 & 44.45409 & 17.12676 & 7.068789 & 17.99532 & 3110.439 & 1.000122 & 251.9827 & 0.804068 \\
\hline 92367 & 182.178 & 50.62972 & 446.2121 & 62.96665 & 117.0642 & 40.98387 & 44.8431 & 37.7873 & 14.78499 & 6.072278 & 16.84215 & 2894.946 & 1.39225 & 256.6269 & 1.007391 \\
\hline 56567 & 38.784 & 42.01425 & 7.7042 & 55.58278 & 90.44495 & 37.21882 & 36.70095 & 24.18319 & 11.38535 & 5.00332 & 20.70616 & 2051.813 & 1.707869 & 267.0518 & 1.095439 \\
\hline 4.57634 & 6051.73 & 179.1788 & 85.7354 & 94.61453 & 218.9322 & 37.64853 & 49.76737 & 40.07259 & 24.07168 & 9.787513 & 25.26909 & 5262.697 & 1.197564 & 541.951 & 0.917779 \\
\hline 689153 & 5823.808 & 186.7586 & 455.8466 & 93.37516 & 201.8041 & 29.03658 & 38.13903 & 35.05152 & 20.9257 & 6.371945 & 24.99137 & 5292.808 & 1.181756 & 514.4058 & 0.77741 \\
\hline 098199 & 5093.018 & 4.2884 & 391.6292 & 76.23312 & 207.411 & 29.50477 & 47.51637 & 35.40117 & 22.70514 & 5.583631 & 23.36254 & 6119.073 & 1.098997 & 541.9395 & 1.024923 \\
\hline 3.3362 & 177.235 & 401.5606 & 406.746 & 101.9527 & 215.4133 & 46.71071 & 62.38219 & 48.91898 & 25.78136 & 6.773203 & 24.26006 & 6600.795 & 1.088886 & 19.2702 & 0.99177 \\
\hline .005445 & 628.704 & 372.0893 & 490.524 & 122.2345 & 191.1526 & 54.36985 & 72.07734 & 59.37047 & 29.90219 & 8.50165 & 22.92401 & 7138.867 & 1.477327 & 18.0779 & 1.309596 \\
\hline 469467 & 7073.602 & 4.1501 & 460.9708 & 6.87432 & 116.0567 & 40.14224 & 50.22291 & 45.23165 & 28.03148 & 5.689396 & 11.51078 & 7237.8 & 1.039383 & 345.9321 & 0.69346 \\
\hline 327583 & 3652.007 & .00413 & 3.0294 & 1.22301 & 102.0183 & 38.45037 & 43.68749 & 40.19669 & 14.8181 & 6.747757 & 26.40218 & 3266.365 & 1.933702 & 405.366 & 1.778872 \\
\hline 430819 & 2274.409 & 3.30207 & 82.2586 & 53.68065 & 97.24682 & 54.29842 & 54.93368 & 37.85717 & 12.5778 & .765715 & 16.87782 & 1827.386 & 1.6076 & 310.7714 & 1.632969 \\
\hline 254809 & 022.409 & 2.6989 & 4.1052 & 70.85006 & 99.21602 & 35.29862 & 42.30314 & 33.91385 & 12.78809 & 716799 & 25.63568 & 2208.248 & 1.608477 & 298.734 & 1.269443 \\
\hline 712145 & 2803.229 & 585.2516 & 68.2534 & 3.71305 & 134.0513 & 42.07354 & 51.31671 & 45.23217 & 11.72709 & 435806 & 23.55438 & 803.116 & 2.192122 & 70.9671 & 1.956167 \\
\hline 4.67768 & 2726.024 & 99.95421 & 559.372 & 59.7221 & 91.43145 & 34.31951 & 39.69208 & 47.54689 & 17.75894 & 333311 & 35.81355 & 4157.124 & 2.271334 & 24.8326 & 1.564953 \\
\hline .941604 & 4378.908 & 8.4657 & 85.0789 & 118.6903 & 168.9606 & 65.06138 & 67.13988 & 55.28125 & 22.89389 & 005363 & 31.6519 & 388.298 & 1.931439 & 62.2851 & 1.671461 \\
\hline 11.67497 & 5693.829 & 86.27572 & 47.1856 & 153.1775 & 156.6302 & 84.96208 & 111.0732 & 43.43458 & 21.45483 & 12.65219 & 36.94147 & 625.333 & 2.86401 & 51.7317 & 2.496048 \\
\hline 7.67161 & 3731.211 & 70.10259 & 797.4378 & 80.89803 & 146.8959 & 61.8526 & 84.80787 & 49.66738 & 20.05304 & . 17968 & 2.13625 & 88.124 & 2.62015 & 8.2832 & 1.574997 \\
\hline .962221 & 2057.988 & 44.02035 & 471.2326 & 59.64631 & 82.48256 & 51.26829 & 4.87797 & 33.54885 & 12.27823 & 24800 & 22.28218 & 57.824 & 1.721486 & 3.4623 & $1.153 / 44$ \\
\hline .872365 & 3679.655 & 54.28298 & 369.7214 & 74.82369 & 142.327 & 49.32488 & 69.25403 & 43.12198 & 18.3941 & 8.422315 & 27.65689 & 2886.209 & 2.20651 & 9.4106 & 329809 \\
\hline 546212 & 814.388 & .04812 & 1089.641 & 59.89568 & 83.05714 & 55.81649 & 70.83238 & 43.18515 & 12.46012 & 13.39052 & 13.08018 & 2797.232 & 2.948773 & 1.5643 & 1.352274 \\
\hline 393184 & 1357.844 & 25.85694 & 408.6821 & 35.20402 & 64.87556 & 32.37574 & 43.83608 & 31.73434 & 9.228643 & 9.403307 & 16.8598 & 1633.944 & 1.620349 & 14.7774 & 0.723826 \\
\hline 036778 & 8136.581 & 96.32035 & 437.4596 & 122.5428 & 169.3195 & 46.10718 & 58.89895 & 34.9194 & 27.08725 & 6.043134 & 14.22605 & 5677.541 & 1.252677 & 517.4201 & 1.207582 \\
\hline 803296 & 8258.885 & 332.6386 & 668.0573 & 131.2237 & 159.1402 & 59.86162 & 72.77884 & 71.27312 & 27.39494 & 6.100013 & 20.44906 & 6438.22 & 1.00178 & 29.6392 & 1.174341 \\
\hline .767463 & 5487.632 & 166.2307 & 438.7657 & 103.2092 & 112.8413 & 33.99194 & 39.56985 & 38.55048 & 19.41862 & 4.237952 & 9.859209 & 4653.669 & 0.70459 & 374.501 & 0.605947 \\
\hline 948387 & 6041.177 & 201.018 & 427.0381 & 107.3528 & 189.4512 & 36.63523 & 50.1345 & 34.53793 & 23.34756 & 10.0468 & 25.28529 & 6037.402 & 1.048539 & 614.8364 & 1.016653 \\
\hline 093661 & 8142.507 & 471.8407 & 465.8869 & 113.0584 & 152.1163 & 45.00509 & 60.80082 & 48.28426 & 32.41784 & 5.673088 & 18.43874 & 9324.462 & 1.328719 & 523.3269 & 0.890445 \\
\hline 856309 & 6481.817 & 3.5714 & 0.4036 & 141.1069 & 125.0641 & 42.64705 & 54.82818 & 51.16678 & 20.08444 & 6.987503 & 21.95529 & 4143.384 & 1.365439 & 312.8548 & 1.271055 \\
\hline 677475 & 71.032 & 5.2766 & 3.9842 & 132.5163 & 144.6162 & 53.25719 & 69.89416 & 61.06027 & 29.16593 & 7.934391 & 24.19557 & 5654.835 & 1.176294 & 335.7836 & 1.194518 \\
\hline 982061 & 33.909 & 4.9638 & 5.6838 & 96.63752 & 182.1001 & 46.00583 & 62.91819 & 51.46053 & 32.9579 & 22559 & 18.81878 & 62.171 & 1.74901 & 74.1592 & 1.181198 \\
\hline .551764 & 60.347 & .5639 & 566 & 44 & 121.7769 & 46.86044 & 1.19505 & 43.07165 & 20.62816 & 4.985649 & 16.20457 & 31.442 & 0.903738 & 3.2523 & 年 \\
\hline .410561 & 1.992 & 739 & 74 & 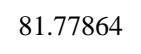 & 83 & 33.89857 & 37.10725 & 30.13886 & 12.40901 & 474039 & 8.992942 & 61.936 & 1.035066 & 7.9986 & 0.467539 \\
\hline 260891 & 6971.68 & .5674 & 7626 & 628 & 6.2471 & 77.35127 & 18028 & 3205 & 855 & & 14.81638 & 2.771 & 2.690856 & .0585 & 1.2915 \\
\hline .610218 & 5808.939 & 8.7662 & 1195 & 176.8483 & 9.9158 & 46.15816 & 2.32101 & 30.28915 & 35.92136 & 47599 & 17.1964 & 25.713 & 0.713859 & 0.9823 & 0.688922 \\
\hline .019053 & 9967.447 & 596.6867 & 2.6531 & 163.372 & 1.5703 & 76.28395 & 9.62673 & 52.81163 & 32.62544 & 7.675878 & 13.81768 & 96.379 & 0.864624 & 17.7376 & 1.21876 \\
\hline .582576 & 81.243 & 195.4868 & .8384 & 69.87721 & 2.8193 & 17.78138 & 21.50058 & 22.42842 & 16.00571 & 84 & 23.88791 & 31.706 & 0.934619 & 0.0854 & 0.616217 \\
\hline 98666 & 1.429 & .6305 & 771.9721 & .5886 & 5068 & 60.19177 & .32356 & 59.16912 & 26.03473 & 31602 & 19.59756 & 269.148 & 1.800797 & 72.9033 & 1.605593 \\
\hline 69929 & 8.299 & 191.8775 & 588.435 & 2325 & 13 & 8527 & 5.2231 & 194 & 29444 & 431 & 30.11491 & 7.209 & 37057 & .5943 & 62941 \\
\hline 99032 & 0.775 & 1994 & 6037 & 287 & 年.5864 & 54.36288 & 94 & 24.50315 & 422 & 017 & (3) & 814 & 1.892778 & 52.2215 & 0.693956 \\
\hline 88014 & 7979.2 & 5765 & 9738 & 58 & & & 00621 & & 731 & & 53 & 08.291 & 596 & 03 & \\
\hline
\end{tabular}




\begin{tabular}{|c|c|c|c|c|c|c|c|c|c|c|c|c|c|c|c|c|}
\hline Salix caprea & 7.181795 & 5153.666 & 400.3934 & 834.1309 & 100.0117 & 225.9756 & 73.66886 & 87.39089 & 65.41807 & 21.63495 & 10.03174 & 33.5801 & 3712.949 & 2.87627 & 365.8531 & 2.153354 \\
\hline Salix fragilis & 4.91742 & 6216.671 & 193.3485 & 573.4351 & 119.8126 & 157.2874 & 68.48809 & 75.56664 & 71.93337 & 25.78902 & 7.156194 & 19.85178 & 6339.924 & 1.94924 & 404.909 & 1.468191 \\
\hline Salix pentandra & 5.747529 & 9359.755 & 213.4004 & 862.4328 & 158.4178 & 271.6649 & 67.07533 & 97.23711 & 57.56606 & 33.00665 & 11.93654 & 32.12427 & 8140.219 & 2.156234 & 868.3432 & 1.833165 \\
\hline Salix purpurea & 4.615952 & 9392.805 & 554.1961 & 755.4252 & 148.4054 & 242.1327 & 81.18269 & 98.35505 & 76.64932 & 36.65271 & 8.524421 & 29.5879 & 9867.961 & 1.752284 & 1079.011 & 1.30499 \\
\hline Salix repens & 7.5326 & 7377.381 & 579.7095 & 436.508 & 112.8081 & 296.6001 & 91.07623 & 107.6241 & 55.66006 & 29.73477 & 6.451181 & 14.33949 & 5524.67 & 1.521649 & 396.7583 & 1.877603 \\
\hline Salix triandra & 4.038336 & 6808.941 & 300.9861 & 839.4107 & 126.8231 & 174.5321 & 61.4648 & 73.9947 & 68.47845 & 29.03301 & 6.615821 & 19.52545 & 7818.192 & 1.159086 & 424.22 & 1.217506 \\
\hline Salix viminalis & 4.301787 & 5999.32 & 362.0131 & 670.0449 & 121.199 & 150.9654 & 63.82114 & 72.92391 & 67.10876 & 23.91047 & 6.45574 & 20.31338 & 5646.995 & 1.453297 & 388.815 & 1.235921 \\
\hline Salvia pratensis & 1.518688 & 3954.463 & 49.623 & 292.5055 & 81.08637 & 66.47982 & 34.12554 & 38.91746 & 25.84183 & 11.74467 & 2.616742 & 8.532521 & 2148.898 & 0.947302 & 273.804 & 0.398519 \\
\hline Sambucus nigra & 6.150362 & 3256.458 & 65.08272 & 715.2192 & 71.6413 & 110.6245 & 42.46169 & 55.19094 & 35.17496 & 14.72226 & 9.612169 & 23.69786 & 3315.054 & 2.646748 & 430.0282 & 1.798967 \\
\hline Sambucus nigra cv. 'Laciniata' L. & 5.845888 & 3267.027 & 45.66323 & 610.4516 & 68.08189 & 116.8399 & 38.7831 & 51.94484 & 34.55622 & 14.99136 & 9.387323 & 23.19797 & 2910.569 & 2.674961 & 322.6025 & 1.526118 \\
\hline Sambucus racemosa & 5.663045 & 2800.191 & 44.65298 & 498.5166 & 66.90915 & 103.6435 & 50.3816 & 65.3726 & 36.88516 & 16.73374 & 9.79862 & 31.14204 & 2376.189 & 2.48492 & 298.6854 & 1.405864 \\
\hline Samolus valerandi & 3.851275 & 6697.555 & 598.6449 & 426.9188 & 113.7816 & 175.0596 & 30.91065 & 58.24833 & 28.15967 & 16.3087 & 4.336102 & 15.82663 & 4723.698 & 1.021208 & 302.0019 & 1.140289 \\
\hline Sanguisorba minor & 2.033121 & 4712.629 & 40.35885 & 354.8481 & 105.7534 & 107.3394 & 56.67731 & 49.99378 & 28.83174 & 18.07639 & 3.697127 & 10.79765 & 2904.346 & 0.585214 & 362.3948 & 0.534025 \\
\hline Sanguisorba officinalis & 5.486384 & 6996.509 & 139.5659 & 373.5977 & 132.6303 & 228.4252 & 42.36356 & 48.14578 & 34.43563 & 24.61716 & 7.342879 & 14.24018 & 5511.163 & 0.911614 & 784.6988 & 1.017588 \\
\hline Sanicula europaea & 2.760627 & 2289.688 & 34.60428 & 250.7821 & 43.5252 & 60.89314 & 32.79941 & 23.86908 & 25.92227 & 9.144014 & 3.152106 & 16.82576 & 1387.936 & 1.718459 & 126.883 & 0.693559 \\
\hline Saponaria officinalis & 5.856338 & 3948.03 & 98.08289 & 554.7065 & 100.9163 & 108.0078 & 45.56305 & 54.21951 & 47.07451 & 16.06609 & 6.623229 & 33.6139 & 2930.435 & 1.678964 & 424.3701 & 1.13637 \\
\hline Saxifraga granulata & 5.542207 & 6558.066 & 82.34598 & 464.8553 & 142.0197 & 289.4582 & 36.9626 & 42.93626 & 23.4549 & 24.32179 & 7.813352 & 7.046144 & 6024.04 & 0.676777 & 929.9275 & 1.01897 \\
\hline Saxifraga tridactylites & 1.263409 & 2181.691 & 37.32958 & 117.8296 & 44.72104 & 87.70856 & 53.12702 & 49.79667 & 31.64174 & 11.74789 & 3.787473 & 8.071973 & 1442.226 & 1.73154 & 236.0691 & 0.61805 \\
\hline Scabiosa columbaria & 1.423014 & 3594.919 & 23.84817 & 270.6433 & 74.16872 & 92.18024 & 53.87856 & 49.39357 & 28.3553 & 10.89642 & 4.113174 & 10.21609 & 2072.556 & 0.510856 & 254.0873 & 0.413945 \\
\hline Scandix pecten-veneris & 3.04303 & 3324.518 & 98.81489 & 790.5246 & 135.2427 & 73.15032 & 26.64661 & 26.8395 & 47.98296 & 9.332109 & 3.043109 & 8.150536 & 1657.87 & 1.304953 & 290.6191 & 0.48871 \\
\hline Schoenoplectus lacustris & 3.766805 & 5199.52 & 377.3646 & 459.1023 & 90.66931 & 187.4212 & 29.39574 & 42.30231 & 32.44106 & 22.793 & 11.09044 & 26.35344 & 5990.92 & 1.527682 & 573.58 & 1.357497 \\
\hline Schoenoplectus lacustris ag. (incl. S. tabernaemontani) & 3.500571 & 5167.765 & 347.2696 & 287.1956 & 89.00612 & 169.1007 & 27.30585 & 38.96122 & 26.04369 & 21.68149 & 10.9676 & 24.13788 & 5583.52 & 1.333933 & 568.2055 & 1.408777 \\
\hline Schoenoplectus tabernaemontani & 6.163111 & 5629.02 & 498.8305 & 477.0395 & 119.703 & 162.5931 & 35.70622 & 59.57678 & 34.26335 & 16.00106 & 5.61878 & 19.5167 & 5251.575 & 1.411258 & 540.9279 & 1.962543 \\
\hline Schoenus nigricans & 4.760468 & 6680.58 & 634.3179 & 319.0259 & 114.0912 & 282.5003 & 73.64047 & 80.47569 & 41.71474 & 19.88866 & 4.464465 & 10.36431 & 4369.719 & 1.030886 & 309.8697 & 1.412243 \\
\hline Scilla non-scripta & 5.467723 & 2873.276 & 34.55368 & 446.1443 & 63.40342 & 92.99888 & 31.21513 & 46.53231 & 16.74252 & 11.47013 & 8.475899 & 23.74209 & 1480.487 & 2.582373 & 261.6683 & 1.434246 \\
\hline Scirpus sylvaticus & 5.849615 & 8022.015 & 132.6995 & 476.0635 & 121.9334 & 190.8765 & 48.83186 & 64.07159 & 32.66691 & 27.22527 & 8.796764 & 28.47629 & 6354.721 & 1.85865 & 610.9633 & 1.052635 \\
\hline Scleranthus annuus & 3.57682 & 3407.009 & 72.34061 & 357.5465 & 91.49322 & 109.236 & 33.97942 & 47.60056 & 39.70843 & 11.39642 & 2.849504 & 11.43729 & 2315.069 & 3.560344 & 474.2577 & 0.947557 \\
\hline Scleranthus perennis & 3.917702 & 2797.457 & 22.76341 & 132.6414 & 69.20752 & 89.80747 & 47.42903 & 54.65312 & 32.45596 & 14.24794 & 3.215569 & 8.248967 & 1969.089 & 0.524403 & 263.9765 & 0.882817 \\
\hline Scleranthus polycarpos & 4.777163 & 2572.523 & 17.76183 & 127.9762 & 60.05335 & 72.73236 & 32.31068 & 39.23494 & 40.43505 & 10.95197 & 2.816158 & 7.114472 & 1739.133 & 1.337529 & 225.0384 & 0.836728 \\
\hline Scrophularia auriculata & 4.957543 & 7824.058 & 252.8761 & 395.768 & 110.461 & 282.0784 & 75.94398 & 99.82547 & 53.87959 & 32.83129 & 7.380576 & 18.10377 & 7572.301 & 0.805476 & 481.6322 & 1.459094 \\
\hline Scrophularia nodosa & 5.647496 & 3882.619 & 73.44997 & 560.9777 & 77.68027 & 133.5266 & 44.91492 & 51.56743 & 45.558 & 17.3872 & 7.935297 & 21.81221 & 2934.716 & 2.320249 & 249.929 & 1.192804 \\
\hline Scrophularia umbrosa & 5.241855 & 8586.087 & 201.9961 & 808.5458 & 140.7916 & 268.4149 & 82.53605 & 106.8955 & 65.86683 & 31.68191 & 11.99728 & 21.21036 & 8293.295 & 1.656726 & 743.7829 & 2.261514 \\
\hline Scrophularia umbrosa s. neesii & 4.371877 & 8351.432 & 261.2899 & 700.8719 & 134.6215 & 277.6292 & 78.4059 & 77.04151 & 76.24092 & 24.79719 & 10.45239 & 18.81558 & 5357.687 & 0.93068 & 699.3756 & 1.425936 \\
\hline Scutellaria galericulata & 4.898232 & 8184.806 & 190.3375 & 487.2363 & 130.5156 & 244.7037 & 64.20636 & 82.8104 & 45.91966 & 27.57697 & 9.058401 & 25.09082 & 5198.99 & 1.091653 & 557.549 & 1.03666 \\
\hline Scutellaria minor & 5.389191 & 7802.021 & 68.65818 & 327.2467 & 55.42401 & 188.6423 & 53.43463 & 79.36262 & 43.36674 & 27.77325 & 7.773498 & 15.18563 & 4038.065 & 1.063562 & 359.1052 & 0.718014 \\
\hline Sedum acre & 1.774901 & 3155.097 & 566.585 & 153.7117 & 67.99345 & 108.9039 & 68.53107 & 61.03932 & 29.73067 & 13.60941 & 3.609077 & 11.44454 & 1771.325 & 1.521116 & 295.1383 & 0.901668 \\
\hline Sedum album & 2.440653 & 4225.986 & 50.60149 & 247.965 & 133.7624 & 125.4054 & 65.25778 & 59.98524 & 47.03427 & 18.63124 & 3.723322 & 27.76927 & 2844.725 & 1.353138 & 463.5486 & 0.849034 \\
\hline Sedum reflexum & 3.433313 & 3191.734 & 27.19471 & 172.6664 & 77.96323 & 95.51344 & 37.41786 & 49.73423 & 33.00508 & 14.51728 & 3.77493 & 11.32376 & 1944.467 & 0.949031 & 256.3032 & 1.184918 \\
\hline Sedum sexangulare & 1.854315 & 3584.905 & 36.11328 & 173.8475 & 91.32814 & 102.6417 & 43.42008 & 48.59605 & 33.30872 & 13.61904 & 3.83345 & 11.65328 & 1704.846 & 1.051711 & 259.644 & 0.867591 \\
\hline Sedum telephium & 9.625456 & 4146.589 & 55.35708 & 708.2994 & 92.40032 & 106.8659 & 40.90797 & 50.27411 & 43.70194 & 16.70465 & 8.111502 & 33.23427 & 3573.172 & 2.319203 & 398.769 & 2.1344 \\
\hline Sedum telephium s. telephium & 7.777676 & 3951.505 & 67.42859 & 506.2147 & 84.67629 & 96.26762 & 39.00832 & 50.88073 & 45.70974 & 16.51985 & 7.006157 & 28.69311 & 3147.502 & 1.675697 & 337.271 & 1.305946 \\
\hline Selinum carvifolia & 5.271894 & 9540.472 & 75.87853 & 509.4829 & 121.9086 & 344.4632 & 85.96232 & 119.3986 & 45.21829 & 33.24751 & 8.735128 & 19.70689 & 7355.182 & 1.953722 & 304.6268 & 1.018143 \\
\hline Senecio aquaticus & 3.40054 & 4811.525 & 364.2453 & 360.1354 & 108.5176 & 147.096 & 24.56992 & 30.80068 & 26.64683 & 18.21318 & 4.767222 & 27.13618 & 4068.834 & 0.846374 & 552.2169 & 0.657769 \\
\hline Senecio erucifolius & 3.718615 & 3999.939 & 400.4876 & 553.4555 & 71.90174 & 95.00361 & 39.34818 & 43.25188 & 36.25545 & 16.49798 & 4.482179 & 14.10886 & 2895.549 & 0.737091 & 268.5288 & 0.518963 \\
\hline Senecio fluviatilis & 4.405921 & 6879.582 & 177.3085 & 695.4488 & 144.9978 & 175.645 & 58.09405 & 69.89932 & 61.5629 & 21.27922 & 5.878544 & 17.82147 & 5153.133 & 0.9092 & 364.137 & 1.055634 \\
\hline Senecio jacobaea s. & 4.366105 & 2647.102 & 67.08415 & 274.6744 & 67.49042 & 109.3287 & 59.27869 & 52.27935 & 29.99038 & 12.06873 & 6.641753 & 19.79995 & 1805.204 & 1.674373 & 304.4236 & 000497 \\
\hline
\end{tabular}



Senecio ovatus

Senecio paludosus

Senecio sylvaticus

Seriphidium maritimu

Sherardia arvensi

Silene conica

Silene dioica

Silene latifolia

Silene nutan

Silene otites

Sinapis arvensis

Sisymbrium altissimum

Sisymbrium officinale

Sium latifolium

Solanum dulcamara

Solanum nigrum

Solanum nigrum s. nigrum

Solidago virgaurea

Sonchus arvensis v. arvensis

Sonchus arvensis v. maritimus

Sonchus asper

oleraceus

Sonchus palustris

Sorbus aucuparia

Sparganium emersum

Sparganium erectum s. erectum

Spergula arvensis

Spergula morisonii

Spergularia marina

Spergularia media s. angustat

Spergularia rubra

Spirodela polyrhiza

Stachys arvensis

tachys officinals

Stachys palustris

Stachys sylvatica

Stellaria aquatica

Stellaria gramine

Stellaria holostea

Stellaria media + S. pallida + S. neglect

Stellaria pallida

\begin{tabular}{|c|c|c|c|c|c|c|c|c|c|c|c|c|c|c|c|}
\hline 394819 & 5049.915 & 267.5209 & 319.5705 & 111.4584 & 117.5337 & 45.09647 & 50.59069 & 28.23654 & 14.55905 & 4.770682 & 13.24107 & 2830.613 & 1.302441 & 374.5934 & 0.781877 \\
\hline 67488 & 2619.598 & 36.96617 & 337.3327 & 52.76603 & 73.48743 & 31.73379 & 27.96371 & 26.34682 & 8.82284 & 6.707155 & 17.36446 & 1257.794 & 1.380544 & 134.3148 & 0.981 \\
\hline 512922 & 653.381 & 232.1345 & 507.7597 & 101.211 & 163.9915 & 41.19995 & 5.33592 & 37.65917 & 21.98371 & 6.416692 & 24.90845 & 6490.742 & 1.089096 & 389.4872 & 0.876731 \\
\hline 46822 & 51.649 & 186.3206 & 24.5953 & 69.9484 & 104.7125 & 60.2015 & 5.15058 & 38.79902 & 3.30185 & 10.0529 & 23.31109 & 2527.178 & 2.112356 & 421.5779 & 2.420958 \\
\hline 05759 & 33.459 & 1.2712 & 404.7632 & 121.1977 & 215.4849 & 28.48676 & .90274 & 29.42144 & 23.94331 & 4.117577 & 28.44291 & 2624.996 & 1.219995 & 404.0965 & 0.795 \\
\hline 2.18073 & 10.856 & 103.7751 & 501.3889 & 96.98786 & 78.44339 & 26.12231 & 7.48419 & 26.22672 & 1.55261 & 3.006464 & 7.906309 & 1961.888 & 3.119771 & 337.0741 & 0.53239 \\
\hline 875096 & 675.666 & 411.4632 & 392.6555 & 127.9563 & 254.9871 & 40.76357 & 0.37783 & 25.80006 & 32.17058 & 3.953601 & 8.394083 & 8762.911 & 0.522932 & 865.191 & 0.517261 \\
\hline 66914 & 2757.52 & 35.819 & 83.41396 & 65.90179 & 8.30773 & 59.20847 & 54.92579 & 35.87675 & 12.41458 & 3.094487 & 9.335319 & 1555.168 & 1.68116 & 259.2614 & 0.662156 \\
\hline 41044 & 3403.881 & 50.35907 & 726.342 & 69.45343 & 98.71764 & 36.52324 & 44.5564 & 34.08747 & 13.72705 & 8.106183 & 20.48666 & 2826.128 & 2.178939 & 321.7857 & 1.197656 \\
\hline 95843 & 2970.291 & 97.44576 & 591.8286 & 78.99755 & 80.61801 & 30.109 & 35.11102 & 39.58287 & 12.13358 & 4.995532 & 21.2222 & 2627.596 & 1.886018 & 353.6428 & 1.246097 \\
\hline 85294 & 2178.248 & 34.02807 & 10.0644 & 68.32742 & 84.48876 & 48.54208 & 40.2471 & 25.17745 & 11.54934 & 4.66697 & 8.415776 & 1552.118 & 1.309197 & 221.3574 & 0.748 \\
\hline 96644 & 2670.117 & 36.5773 & 109.0738 & 67.61323 & 98.5136 & 55.61261 & 48.76796 & 24.24227 & 12.639 & 3.521305 & 7.548773 & 1575.806 & 1.184044 & 234.5984 & 0.612125 \\
\hline 318763 & 699.117 & 69.61533 & 484.4219 & 104.254 & 83.38824 & 51.86147 & 39.01737 & 35.96145 & 15.40403 & 5.552028 & 14.46762 & 2838.068 & 0.568135 & 380.8125 & 0.554099 \\
\hline 63445 & 5569.534 & 135.572 & 702.5216 & 127.465 & 122.2334 & 30.52742 & 35.91869 & 44.99721 & 20.70831 & 4.701686 & 24.3851 & 3562.908 & 2.404772 & 678.638 & 0.702882 \\
\hline 504128 & 952.644 & 161.9311 & 298.0976 & 146.3579 & 125.238 & 68.84994 & 62.92006 & 30.68666 & 14.05516 & 2.923528 & 16.96699 & 2351.324 & 1.330421 & 495.9818 & 0.747182 \\
\hline 173333 & 47.539 & 112.0526 & 6.9253 & 120.6476 & 96.73959 & 30.51097 & 36.34123 & 41.49552 & 13.59543 & 4.232775 & 20.50605 & 2657.899 & 1.818609 & 392.496 & 0.983853 \\
\hline 535375 & 5228.821 & 224.7799 & 03.2472 & 89.39159 & 182.2047 & 29.79854 & 43.28069 & 28.94985 & 20.74869 & 10.43309 & 23.7348 & 5619.239 & 1.115566 & 54.2479 & 0.867676 \\
\hline 291341 & 676.417 & 297.9006 & 6.2383 & 139.1088 & 304.5362 & 72.0999 & 90.45555 & 64.13222 & 32.98527 & 11.17712 & 19.45441 & 8926.661 & 1.183399 & 923.3991 & 1.681471 \\
\hline 363981 & 762.121 & 359.47 & 46.2759 & 171.9992 & 157.9928 & 61.57082 & 94.2003 & 49.28199 & 16.95149 & 4.880741 & 21.70202 & 3639.522 & 1.888472 & 19.1525 & 2.516182 \\
\hline 141577 & 5945.582 & 360.2629 & 57.0755 & 184.8862 & 162.7969 & 59.90477 & 85.76619 & 63.81733 & 21.81658 & 7.75175 & 21.76016 & 4358.785 & 2.356925 & 63.2778 & 1.565982 \\
\hline 946742 & 55.914 & 44.9415 & 4.2549 & 73.65349 & 101.8648 & 45.03841 & 65.56672 & 47.23928 & 13.18104 & 14.27073 & 31.42501 & 2473.464 & 2.855074 & 83.6629 & 2.191565 \\
\hline 4.89864 & 702.506 & 666.5582 & 66.2715 & 161.2973 & 294.6815 & 67.86636 & 69.19353 & 66.97491 & 25.14695 & 6.867012 & 24.64123 & 4325.188 & 2.040348 & 25.7744 & 0.924423 \\
\hline 847382 & 4655.003 & 630.0911 & 12.8339 & 133.9916 & 167.1454 & 48.59545 & 53.84978 & 54.88697 & 23.3277 & 7.259902 & 22.97566 & 4754.77 & 1.846671 & 2.4321 & .04106 \\
\hline 608074 & 17.764 & 663.1986 & 8.7176 & 185.9469 & 335.1662 & 78.75638 & 75.5315 & 61.99795 & 22.39068 & 6.060097 & 24.67485 & 2702.096 & 1.751664 & 61.4574 & 0.790315 \\
\hline 492787 & 378.248 & 498.0201 & 3.2645 & 139.6488 & 145.5669 & 44.43149 & 54.00489 & 58.04146 & 23.28583 & 6.374425 & 19.5059 & 152.568 & 1.990047 & 41 & 1.048171 \\
\hline 552072 & 5333.584 & 610.2913 & 5.0595 & 133.7714 & 146.3383 & 43.53866 & 54.35242 & 57.72483 & 21.26757 & 79789 & 21.71801 & 556.386 & 2.033837 & 345 & 1.204351 \\
\hline 59103 & 7317.202 & 228.6732 & 4.6896 & 97.28691 & 210.6659 & 46.52131 & 59.39193 & 40.38272 & 22.7606 & 8.906551 & 21.18713 & 5486.107 & 0.762267 & 8.9529 & 1.324563 \\
\hline 787836 & 3078.267 & 49.14705 & 549.1515 & 73.13207 & 116.149 & 61.33139 & 83.12336 & 44.03868 & 17.58151 & 9.767079 & 31.34629 & 3038.78 & 2.478878 & 29.1105 & 1.435459 \\
\hline 458898 & 4578.419 & 193.3977 & 362.5695 & 83.85235 & 183.1046 & 24.25368 & 26.75478 & 32.71926 & 17.93866 & 11.62845 & 18.04092 & 4953.338 & 1.211026 & 27.3711 & 0.736703 \\
\hline .054658 & 6822.842 & 182.1055 & 432.3425 & 113.579 & 185.1221 & 33.24698 & 46.47859 & 29.03064 & 23.15139 & 10.7081 & 24.55795 & 5804.992 & 1.486039 & 8.5631 & 1.133809 \\
\hline .525292 & 6315.314 & 217.5483 & 463.2647 & 104.6051 & 197.4132 & 31.01678 & 41.41849 & 31.83537 & 19.26549 & 8.733164 & 26.23171 & 5288.193 & 1.381182 & 41.6493 & 0.898063 \\
\hline .991292 & 4269.012 & 88.20474 & 18.1244 & 109.1541 & 121.387 & 34.71186 & 49.88505 & 46.36554 & 13.94811 & 3.417864 & 14.05602 & 2972.031 & 2.732879 & 499.7243 & 1.016374 \\
\hline .747724 & 848.3835 & 17.89511 & 75.3502 & 28.18927 & 23.26725 & 48.35658 & 67.88303 & 38.36376 & 6.715434 & 5.235901 & 3.869098 & 969.3534 & 0.369914 & 171.9129 & 0.752291 \\
\hline 2.08296 & 5317.304 & 593.6104 & 4.1762 & 77.74064 & 32.2283 & 24.11794 & 25.29584 & 38.94996 & 19.64373 & 4.960001 & 15.03618 & 137.592 & 1.649618 & 50.6713 & 0.819154 \\
\hline 335637 & 5747.119 & 574.1256 & 2.6363 & 68.6759 & 159.8996 & 25.92947 & 26.83542 & 32.67147 & 20.96368 & 4.775704 & 15.54732 & 881.235 & 1.74853 & 83.0327 & 0.712174 \\
\hline 513191 & 31.415 & 106.6312 & 8.7435 & 114.2315 & 5.1338 & 70.77906 & 01.167 & 60.03227 & 19.17708 & 4.057633 & 13.43416 & 49.357 & 3.484566 & 95.4341 & 1.371737 \\
\hline 73784 & 1.622 & .1828 & .1147 & 76.61782 & 7.3589 & 19.15936 & 6358 & 23.56705 & 17.95243 & 7.686872 & 25.374 & 540.506 & 1.162573 & 8.7119 & 0.691598 \\
\hline 55328 & 2747.062 & 99.39203 & 17 & 80.61844 & 18 & 40685 & 433 & 67 & 10.46148 & 2.761164 & 14.64415 & 30.394 & 1.684999 & 6191 & 0.779638 \\
\hline 570324 & 60.251 & 54566 & 3.5088 & 0.91499 & 17661 & 76856 & .79578 & 29.14503 & .808961 & & 13.41985 & 32.901 & 0.492017 & 4.8688 & 2.224075 \\
\hline .256174 & 7457.83 & 5.4938 & 6.4228 & 120.4849 & 2.3557 & 36657 & 0.03598 & 4801 & 31.58995 & 1.10544 & 20.77887 & 85.491 & 0.997501 & 8.2189 & 1.089571 \\
\hline .312567 & 3104.626 & 53.05234 & 3.0753 & 58.37322 & .01887 & 39.55985 & 7.49537 & 36.18201 & 13.08734 & 332034 & 15.31108 & 01.037 & 0.982572 & 62.4269 & 0.849571 \\
\hline 5.51958 & 9.191 & .8105 & .3419 & .3476 & 7.3441 & 59.97955 & 96484 & 1121 & 32.9184 & 45471 & 20.19491 & 521 & 1.557928 & 49.962 & 1.39779 \\
\hline .079206 & 39.594 & .8474 & 5.5514 & 109.9356 & 5.2977 & 50.44875 & 91265 & 36.76455 & 29.69642 & 18 & 14.85504 & 866 & 1.474754 & 7.4636 & 1.017644 \\
\hline 5.558191 & 3338.454 & 37.42015 & .5069 & 67.37204 & .55215 & 37.1222 & 58479 & 31.3529 & 12.21255 & 68395 & 23.15051 & 0.705 & 2.687301 & 83.5637 & 1.347239 \\
\hline 7.16787 & 7.873 & 116.4099 & 344 & 界1 & . .0039 & 502 & 83 & 272 & 14 & & 991 & & .029707 & 1.3917 & 1.034629 \\
\hline 2623 & 227 & 249.876 & 07 & 12 & 61 & 106 & 7727 & & 591 & & & 06 & 765 & 34.439 & \\
\hline
\end{tabular}




\begin{tabular}{|c|c|c|c|c|c|c|c|c|c|c|c|c|c|c|c|c|}
\hline Stellaria palustris & 3.573614 & 4449.932 & 192.8813 & 371.8986 & 104.168 & 192.6063 & 28.79173 & 38.6066 & 29.24189 & 19.10786 & 8.163197 & 22.84742 & 3502.743 & 1.006711 & 585.6547 & 0.690034 \\
\hline Stellaria uliginosa & 5.838572 & 7943.809 & 137.0747 & 468.2216 & 136.9251 & 191.417 & 47.45092 & 64.56939 & 33.73909 & 25.46752 & 7.777111 & 24.89942 & 6184.684 & 1.700441 & 501.9581 & 1.117777 \\
\hline Stratiotes aloides & 3.235892 & 5081.95 & 162.5452 & 517.8279 & 78.9322 & 142.1412 & 25.21723 & 39.33696 & 20.02357 & 19.45731 & 10.29064 & 23.03041 & 5115.803 & 1.309937 & 506.8231 & 0.768415 \\
\hline Succisa pratensis & 5.06143 & 6207.207 & 65.84086 & 498.7377 & 73.28565 & 211.8623 & 54.32315 & 63.96956 & 30.43191 & 24.27664 & 5.874955 & 10.18981 & 5300.528 & 0.913307 & 343.6752 & 1.105565 \\
\hline Symphytum officinale & 5.430133 & 8136.616 & 174.8147 & 746.7668 & 122.1749 & 192.2936 & 51.32315 & 62.19622 & 55.63652 & 27.59558 & 8.576022 & 24.17873 & 5398.703 & 1.073413 & 365.372 & 1.134248 \\
\hline Tanacetum vulgare & 6.132093 & 3856.729 & 118.1101 & 530.0126 & 101.1069 & 104.7381 & 34.33434 & 44.76551 & 44.42046 & 14.67342 & 6.051277 & 20.6838 & 3088.718 & 1.748393 & 370.3612 & 1.29541 \\
\hline Taraxacum commixtum & 1.132812 & 2906.91 & 33.02691 & 132.4041 & 75.35908 & 90.47746 & 62.03518 & 86.48337 & 40.47359 & 15.63023 & 4.041299 & 9.814819 & 1798.659 & 1.641123 & 271.0988 & 0.932089 \\
\hline Taraxacum lacistophyllum & 4.557188 & 2362.366 & 37.72894 & 202.8717 & 67.71621 & 91.34616 & 51.30372 & 49.33254 & 43.70196 & 11.7412 & 3.577463 & 13.34155 & 1719.96 & 1.82588 & 259.4202 & 1.714084 \\
\hline Taraxacum nordstedtii & 2.763468 & 7746.596 & 61.67943 & 452.4274 & 75.69185 & 138.8267 & 44.74296 & 57.44995 & 22.16055 & 24.55686 & 4.44583 & 7.414856 & 5960.868 & 0.752367 & 569.7568 & 0.529113 \\
\hline Taraxacum obliquum & 2.168177 & 2099.858 & 100.122 & 171.1283 & 55.93922 & 83.54372 & 46.61439 & 36.41845 & 25.8829 & 10.48869 & 4.542595 & 7.317146 & 1183.067 & 1.728885 & 172.4781 & 0.968749 \\
\hline Taraxacum rubicundum & 2.48628 & 2575.385 & 29.82767 & 180.1364 & 58.86143 & 97.82744 & 51.27106 & 47.62199 & 31.33295 & 13.79494 & 4.342283 & 10.03927 & 1701.267 & 1.617931 & 248.0478 & 0.980185 \\
\hline Taraxacum sectie Celtica & 6.510099 & 9792.485 & 176.436 & 447.8553 & 189.8887 & 304.835 & 111.8855 & 132.5225 & 80.40776 & 40.65062 & 7.944669 & 16.02989 & 7810.753 & 1.226656 & 653.1645 & 2.231087 \\
\hline Taraxacum sectie Erythrosperma & 3.064156 & 2600.43 & 41.02745 & 214.7099 & 70.81053 & 96.99988 & 51.68369 & 46.20579 & 26.39306 & 10.16891 & 5.516002 & 11.31757 & 1643.088 & 1.702588 & 250.1986 & 1.041172 \\
\hline Taraxacum sectie Hamata & 6.187659 & 5715.336 & 72.24526 & 494.2933 & 106.481 & 120.2276 & 40.8735 & 52.44687 & 33.6029 & 19.73913 & 6.491263 & 15.8235 & 3863.753 & 1.331647 & 384.8018 & 1.258673 \\
\hline Taraxacum sectie Palustria & 3.483011 & 6860.549 & 673.9645 & 414.4407 & 112.6864 & 199.979 & 39.15525 & 49.08415 & 24.72475 & 25.38187 & 6.028399 & 11.42713 & 6104.629 & 0.696831 & 550.5864 & 1.038429 \\
\hline Taraxacum sectie Ruderalia & 7.257669 & 6985.804 & 306.7701 & 559.9368 & 152.5711 & 145.1943 & 51.05998 & 62.62615 & 49.37118 & 23.45327 & 6.00328 & 19.13488 & 5586.876 & 1.379771 & 642.53 & 1.428702 \\
\hline Taraxacum taeniatum & 2.916276 & 1655.061 & 23.61403 & 197.1396 & 50.77115 & 61.28707 & 46.85404 & 41.63701 & 44.35481 & 9.027877 & 5.80111 & 11.31069 & 1407.164 & 1.948608 & 253.2013 & 1.401828 \\
\hline Taraxacum tortilobum & 2.723978 & 3055.432 & 35.93345 & 172.3915 & 84.36939 & 97.39003 & 47.94797 & 51.01934 & 30.71862 & 13.35529 & 4.061228 & 13.45522 & 2018.829 & 1.686052 & 296.7165 & 1.257607 \\
\hline Taxus baccata & 6.52369 & 2567.623 & 30.44747 & 574.1079 & 60.96853 & 87.90087 & 39.66052 & 58.73398 & 23.40323 & 14.71442 & 9.837934 & 28.59431 & 2016.284 & 2.59197 & 463.6238 & 1.471084 \\
\hline Teesdalia nudicaulis & 4.888622 & 2561.246 & 29.43921 & 228.0027 & 54.71602 & 72.35294 & 68.29117 & 72.57472 & 35.00694 & 10.87563 & 3.288807 & 11.30837 & 1903.998 & 2.675371 & 334.9221 & 0.968494 \\
\hline Tephroseris palustris & 4.965148 & 8853.558 & 609.1867 & 973.0362 & 143.2594 & 276.4333 & 52.69203 & 73.22392 & 55.68032 & 33.62801 & 11.15574 & 25.333 & 9465.545 & 1.213219 & 715.2776 & 0.934945 \\
\hline Teucrium scordium & 1.797123 & 3012.702 & 60.3997 & 261.6841 & 46.13115 & 82.34039 & 18.08386 & 18.80615 & 13.51674 & 9.800925 & 2.979298 & 6.522987 & 2401.116 & 0.885946 & 198.6269 & 0.499377 \\
\hline Teucrium scorodonia & 7.713404 & 2550.814 & 40.90748 & 980.476 & 58.35605 & 91.32607 & 40.79091 & 61.52217 & 26.96556 & 13.90699 & 12.46008 & 26.53636 & 2768.071 & 2.751552 & 486.8694 & 1.892016 \\
\hline Thalictrum flavum & 3.774977 & 6907.705 & 155.2391 & 523.4673 & 123.2926 & 148.8738 & 42.10972 & 46.11288 & 32.31854 & 21.58325 & 6.467681 & 29.28186 & 4253.554 & 1.318334 & 442.3124 & 0.922026 \\
\hline Thalictrum minus & 3.117485 & 3768.853 & 44.28033 & 345.5412 & 74.85128 & 72.53888 & 38.29293 & 45.30579 & 40.67341 & 14.32408 & 4.460582 & 21.16961 & 2331.264 & 1.243644 & 367.0971 & 0.551555 \\
\hline Thalictrum minus s. dunense & 4.607318 & 2917.474 & 20.47742 & 178.5969 & 65.02559 & 90.61228 & 52.42302 & 36.64485 & 42.44913 & 15.24188 & 5.047879 & 15.70706 & 1938.462 & 2.49885 & 285.0015 & 1.817535 \\
\hline Thelypteris palustris & 4.479499 & 7396.62 & 147.9043 & 390.1365 & 107.4812 & 202.21 & 53.76386 & 71.95303 & 31.2248 & 23.06897 & 7.26424 & 30.97841 & 3688.228 & 1.239242 & 690.9742 & 0.859451 \\
\hline Thlaspi arvense & 3.041576 & 3626.348 & 85.63552 & 904.2219 & 116.5796 & 107.0356 & 24.32598 & 28.23372 & 44.02616 & 13.58236 & 3.961307 & 17.83373 & 2918.732 & 1.65408 & 278.2231 & 0.606887 \\
\hline Thymus pulegioides & 2.907451 & 3029.049 & 38.93659 & 211.5719 & 80.51792 & 89.4624 & 51.40478 & 47.66065 & 27.0343 & 13.29784 & 4.22944 & 10.0487 & 1746.86 & 1.007671 & 249.786 & 0.901463 \\
\hline Thymus serpyllum & 6.502515 & 3733.004 & 30.91839 & 202.8418 & 87.14204 & 109.0783 & 56.10061 & 71.82788 & 33.06497 & 17.94968 & 4.124892 & 10.53497 & 2365.027 & 0.474358 & 298.6542 & 1.668832 \\
\hline Tilia cordata & 4.211219 & 2739.128 & 29.45918 & 493.8494 & 54.1578 & 76.85142 & 32.38313 & 36.37924 & 25.45465 & 9.224013 & 7.133954 & 19.92458 & 1073.042 & 1.779086 & 240.1227 & 1.012399 \\
\hline Tilia platyphyllos & 5.766095 & 2693.224 & 28.01272 & 496.0903 & 60.64361 & 83.6356 & 31.5027 & 37.04269 & 18.55415 & 9.052032 & 11.80406 & 20.54729 & 1315.098 & 2.926072 & 176.2203 & 1.357278 \\
\hline Torilis japonica & 7.094739 & 3754.022 & 61.07299 & 631.5876 & 84.95102 & 90.66207 & 37.01635 & 40.8499 & 39.33158 & 14.2308 & 6.388725 & 23.587 & 2750.315 & 1.605041 & 366.4281 & 1.290996 \\
\hline Torilis nodosa & 1.577877 & 4048.915 & 205.0557 & 465.9683 & 110.2001 & 100.9899 & 24.59872 & 27.22214 & 23.27767 & 9.640661 & 2.989338 & 8.346437 & 2168.137 & 1.435748 & 273.7892 & 0.683656 \\
\hline Tragopogon porrifolius & 1.470175 & 2539.434 & 110.839 & 264.1659 & 48.78375 & 45.28025 & 13.089 & 14.15191 & 13.23482 & 5.611928 & 1.401233 & 5.498803 & 1217.778 & 0.264563 & 110.4077 & 0.201237 \\
\hline Tragopogon pratensis & 2.246545 & 5203.006 & 61.80303 & 384.1987 & 107.1335 & 89.42003 & 43.0988 & 47.41027 & 31.50329 & 17.42941 & 3.83479 & 10.60588 & 3526.53 & 0.958507 & 407.7988 & 0.530407 \\
\hline Tragopogon pratensis s. minor & 3.030672 & 6285.043 & 88.99936 & 258.2301 & 136.124 & 138.6376 & 84.84209 & 109.8775 & 48.54471 & 20.43626 & 5.796242 & 22.00851 & 2655.326 & 1.226726 & 412.9513 & 0.912442 \\
\hline Tragopogon pratensis s. orientalis & 1.598312 & 7939.629 & 71.64977 & 465.6507 & 89.88294 & 74.18342 & 54.83797 & 41.3693 & 23.47037 & 13.29583 & 4.003676 & 11.12651 & 2884.837 & 0.724696 & 284.3891 & 0.784155 \\
\hline Tragopogon pratensis s. pratensis & 2.506485 & 3968.669 & 61.88653 & 403.5135 & 73.11985 & 74.17997 & 30.14001 & 31.99157 & 19.71133 & 10.56002 & 2.674059 & 8.047463 & 2492.188 & 0.74588 & 248.2248 & 0.557846 \\
\hline Trichophorum cespitosum & 6.638455 & 3193.648 & 17.12225 & 799.0751 & 62.87043 & 116.189 & 83.27655 & 131.1687 & 32.85748 & 22.53423 & 6.643324 & 5.659097 & 3607.851 & 2.178834 & 249.4477 & 0.752887 \\
\hline Trichophorum cespitosum s. germanicum & 6.576933 & 2718.903 & 20.9405 & 671.1647 & 57.57751 & 86.09369 & 77.1002 & 131.689 & 47.71362 & 16.89731 & 7.445146 & 6.217912 & 3296.192 & 2.622498 & 282.0855 & 0.728836 \\
\hline Trifolium arvense & 3.959722 & 3836.668 & 139.8473 & 197.6191 & 106.423 & 119.6299 & 52.59709 & 56.12623 & 30.02964 & 14.53828 & 3.503692 & 11.09566 & 2031.969 & 1.630089 & 297.2051 & 1.28575 \\
\hline Trifolium campestre & 3.018383 & 4313.479 & 379.7677 & 210.0361 & 119.1622 & 127.5065 & 52.59683 & 58.93435 & 33.88565 & 16.51671 & 3.753317 & 11.14533 & 2432.726 & 1.422649 & 335.2136 & 0.948067 \\
\hline Trifolium fragiferum & 2.573518 & 6395.609 & 600.125 & 422.8363 & 95.2246 & 157.5909 & 31.82567 & 32.4766 & 38.58017 & 15.27381 & 4.917279 & 10.13427 & 4094.748 & 0.923244 & 398.0436 & 0.8401 \\
\hline Trifolium hybridum & 4.524807 & 8118.21 & 475.9887 & 457.5779 & 142.247 & 197.3984 & 49.46642 & 58.38534 & 38.75546 & 24.18276 & 6.976911 & 13.04308 & 5246.995 & 1.140712 & 416.8983 & 1.238812 \\
\hline Trifolium medium & 4.407665 & 6253.754 & 84.18598 & 542.6593 & 149.1004 & 137.568 & 47.64893 & 60.50675 & 31.61082 & 22.26925 & 6.374529 & 15.004 & 6276.473 & 0.608637 & 474.011 & 1.334209 \\
\hline
\end{tabular}


Trifolium micranthum

Trifolium pratense

Trifolium scabru

Trifolium striatum

Triglochin maritima

Triglochin palustris

Tripleurospermum maritimum

Trisetum flavescens

eraria guttata

Typha angustifolia

Typha latifolia

Ulmus glabra

Urtica dioica

Urtica urens

Utricularia minor

Vaccinium myrtillus

Vaccinium uliginosum

Vaccinium vitis-idaea

Valeriana dioica

aleriana officinalis

Valerianella dentata

Valerianella locusta

Verbascum densiflorum

Verbascum nigrum

Verbena officinalis

Veronica agrestis

Veronica anagallis-aquatica

Veronica arvensis

Veronica austriaca s. teucrium

Veronica beccabunga

Veronica catenat

Veronica chamaedrys

Veronica filitorm!

Veronica hederifolia

Veronica hederifolia s. hederifolia

Veronica longifolia

monica montana

eronica officinalis

ronica persica

Veronica scutellata

Viburnum opulus

Vicia cracca

Vicia hirsuta

\begin{tabular}{|c|c|c|c|c|c|c|c|c|c|c|c|c|c|c|c|}
\hline 400666 & 6090.606 & 113.5208 & 232.5151 & 131.6802 & 175.4182 & 72.01425 & 54.79566 & 42.45273 & 21.14683 & 4.469159 & 10.51419 & 3751.1 & 0.908345 & 343.2776 & 1.00007 \\
\hline 332277 & 6414.707 & 511.0239 & 404.1768 & 107.8669 & 124.6701 & 36.90465 & 44.10993 & 29.31925 & 20.23259 & 4.415242 & 11.50871 & 4444.426 & 0.836554 & 383.7427 & 0.94271 \\
\hline 491159 & 462.074 & 718.7662 & 109.558 & 90.6446 & 104.6537 & 54.61397 & 62.84418 & 41.30552 & 13.84573 & 3.177006 & 10.79525 & 1904.936 & 0.955647 & 266.8663 & 0.9558 \\
\hline 355333 & 166.767 & 46.79708 & 153.8606 & 91.12939 & 103.2402 & 41.23228 & 1.22195 & 30.62781 & 11.56312 & 3.437905 & 9.204666 & 1821.607 & 1.35013 & 240.2172 & 801 \\
\hline 73662 & 136.223 & 558.2993 & 399.3271 & 87.51071 & 165.8065 & 31.40715 & 29.68055 & 40.41345 & 14.12068 & 5.91387 & 12.03523 & 3962.665 & 0.747858 & 272.5702 & .83 \\
\hline 73063 & 997.134 & 664.4905 & 53.7825 & 100.8655 & 141.8986 & 29.59667 & 30.99655 & 34.39782 & 16.79505 & 5.689561 & 12.11865 & 6770.813 & 0.985968 & 612.3369 & 1.671224 \\
\hline 42115 & 165.738 & 614.1559 & 82.1624 & 147.0522 & 163.2114 & 38.05632 & 45.13333 & 46.23687 & 21.12438 & 5.425922 & 19.22268 & 4086.849 & 2.360656 & 543.2847 & 1.025 \\
\hline 11378 & 4280.801 & 62.8529 & 424.0279 & 88.26703 & 85.49368 & 36.69346 & 38.96089 & 24.33712 & 12.97814 & 2.671038 & 8.092377 & 2999.545 & 0.742222 & 315.7041 & 0.592 \\
\hline 74909 & 223.851 & 32.12474 & 22.4859 & 48.52268 & 86.2466 & 57.4949 & 81.23342 & 48.67663 & 12.44383 & 3.43473 & 5.498372 & 1637.763 & 0.71786 & 219.9309 & $0.58:$ \\
\hline 133589 & 6599.234 & 277.8049 & 397.7742 & 121.3367 & 182.0872 & 36.50549 & 55.1179 & 31.19713 & 24.09958 & 9.772979 & 28.22918 & 6088.427 & 1.319168 & 653.7583 & 1.299374 \\
\hline 45128 & 7712.375 & 331.9622 & 481.593 & 125.3801 & 214.7728 & 41.32164 & 58.1178 & 39.99241 & 27.75853 & 11.60661 & 31.10827 & 6705.565 & 1.769786 & 49.5532 & 1.6697 \\
\hline 27482 & 3072.567 & 46.44298 & 593.5603 & 63.07471 & 83.99353 & 54.63747 & 44.09593 & 22.21986 & 10.68201 & 8.660932 & 19.00672 & 2015.21 & 3.486879 & 277.8813 & 1.600 \\
\hline 87172 & 2856.621 & 41.73674 & 32.0323 & 60.29517 & 71.6728 & 34.01978 & 32.49327 & 19.67841 & 9.698363 & 5.740231 & 16.10291 & 1476.225 & 2.217017 & 203.9188 & 1.722425 \\
\hline 925964 & 988.034 & 154.4162 & 3.5579 & 113.6843 & 176.0539 & 54.76766 & 67.70069 & 53.05411 & 26.39783 & 9.384757 & 23.77217 & 5643.031 & 1.795511 & 577.5618 & 1.782511 \\
\hline 97261 & 105.966 & 224.8927 & 12.4469 & 177.133 & 135.7688 & 42.06312 & 52.07284 & 39.77026 & 14.04689 & 3.761937 & 17.68017 & 3733.098 & 1.782809 & 652.8895 & 1.44407 \\
\hline 13762 & 445.578 & 172.6858 & 8.3802 & 134.624 & 146.1273 & 26.51439 & 41.11166 & 24.76359 & 23.54068 & 8.766176 & 26.90376 & 3420.973 & 2.146175 & 90.9577 & 0.848117 \\
\hline 299478 & 32.434 & 149.6762 & 3.4115 & 95.83579 & 158.8702 & 26.07165 & 39.67247 & 28.71268 & 21.1714 & 10.31032 & 30.27346 & 5337.929 & 1.549475 & 41.9384 & 0.9895 \\
\hline 363413 & 1367.96 & 32.69199 & 1.9607 & 44.23552 & 68.14571 & 49.27 & 61.08305 & 42.62671 & 13.00321 & 9.724547 & 13.18208 & 2035.492 & 2.125031 & 96.6878 & 0.823501 \\
\hline 522791 & 54.082 & 38.56211 & 061.564 & 68.10606 & 130.7873 & 104.993 & 141.2733 & 39.42075 & 20.39372 & 11.4746 & 7.182981 & 3548.867 & 2.168327 & 32.1087 & .331172 \\
\hline 192393 & 006.394 & 32.7139 & 68.4991 & 52.6763 & 79.89795 & 63.99659 & 73.87228 & 4.2575 & 14.3379 & 9.665012 & 8.870905 & 805.262 & 2.051545 & 23.3923 & 963035 \\
\hline 098848 & 41.025 & 103.8422 & 407.8074 & 71.28225 & 184.1703 & 36.5052 & 44.27861 & 5.0903 & 18.13937 & 5.794015 & 21.73447 & 890.66 & 1.247444 & 407.1724 & .557632 \\
\hline 56085 & 73.623 & 176.8418 & 81.0097 & 119.352 & 227.5329 & 62.23556 & 74.10443 & 57.67605 & 29.882 & 238455 & 25.98084 & 6345.565 & 1.309353 & 52.5817 & 19563 \\
\hline 416345 & 7661.329 & 70.48924 & 505.2094 & 121.1221 & 178.7918 & 62.54054 & 53.74784 & 60.55046 & 40.116 & 2.900814 & 14.29277 & 7946.927 & 2.535282 & 33.5078 & 1.027567 \\
\hline 393049 & 5179.39 & 87.76025 & 406.9123 & 136.9959 & 111.0877 & 61.65869 & 0.16491 & 56957 & 20.86022 & 26302 & 13.27576 & 3368.493 & 1.789761 & 3.0681 & 600933 \\
\hline 35445 & 3337.293 & 128.4311 & 717.7645 & 136.3721 & 109.2292 & 39.60961 & 50.94791 & 43.83631 & 11.34221 & 658996 & 32.73027 & 3932.08 & 1.894184 & 6.7549 & 433156 \\
\hline 681701 & 3432.283 & 82.15125 & 625.7187 & 88.7621 & 80.81612 & 35.06687 & 39.98782 & 40.72859 & 11.57483 & 5.758896 & 32.92389 & 2465.561 & 1.676925 & 53.3777 & 091464 \\
\hline 383796 & 5135.244 & 268.696 & 550.285 & 120.2576 & 132.4628 & 43.08108 & 49.34329 & .27628 & 15.7965 & 4.808327 & 21.92903 & 3462.951 & 1.200579 & 2.8456 & 83 \\
\hline 229033 & 6264.19 & 104.4766 & 578.942 & 182.2936 & 151.9268 & 32.86401 & 39.30955 & 40.10004 & 16.23639 & 3.556689 & 15.40964 & 3775.826 & 1.798813 & 28.1703 & 69 \\
\hline 901049 & 8126.507 & 318.623 & 861.1847 & 118.6209 & 231.4177 & 45.17905 & 64.64999 & 46.60623 & 31.79978 & 6.203212 & 21.21879 & 8410.898 & 1.325935 & 593.3212 & 0.970459 \\
\hline 344286 & 5317.371 & 61.32999 & 293.7443 & 128.924 & 134.0995 & 71.22921 & 67.21824 & 35.71967 & 16.55475 & 4.328209 & 11.85833 & 2901.728 & 2.522701 & 25.4553 & 186 \\
\hline 635278 & 3128.473 & 48.68064 & 243.3629 & 69.96936 & 56.78553 & 31.72859 & 33.27124 & 31.28261 & 10.95576 & 2.331316 & 8.138467 & 1647.453 & 1.034385 & 241.633 & 0.50145 \\
\hline .960826 & 6524.174 & 224.2196 & 433.3823 & 106.8314 & 237.3328 & 44.59187 & 62.25856 & 44.24173 & 25.99309 & 6.589595 & 21.89928 & 6747.818 & 1.161929 & 546.6697 & 1.178673 \\
\hline 354239 & 7108.136 & 351.3008 & 488.689 & 101.4507 & 205.5948 & 38.8494 & 49.05838 & 44.41647 & 28.85858 & 5.286512 & 14.53216 & 7261.623 & 0.910259 & 551.8831 & 0.852637 \\
\hline 44106 & 5839.082 & 55.37235 & 619.0358 & 110.3203 & 120.5949 & 54.85348 & 62.55296 & 48.65396 & 23.89177 & 5.69689 & 25.53018 & 4162.375 & 1.390189 & 443.25 & .446201 \\
\hline 011855 & 7547.722 & 151.3321 & 556.9399 & 147.2061 & 187.3244 & 62.10538 & 74.87622 & 57.21635 & 24.86361 & 6.457981 & 19.53626 & 5036.261 & 1.526342 & 479.5622 & 1.479967 \\
\hline 286126 & 2989.002 & 63.02788 & 1091.037 & 72.38174 & 78.90885 & 37.59293 & 39.45124 & 18.81787 & 12.29293 & 7.92026 & 28.34662 & 2293.057 & 1.642084 & 51.4036 & 1.366199 \\
\hline 86735 & 61.513 & .31231 & 99. & 3.5331 & 104.3664 & 44.49788 & 40.50782 & 20.93672 & 18.17223 & 7.246414 & 29.13245 & 949.16 & 3.146438 & 2.8151 & 1.24 \\
\hline 28036 & 8170.677 & 213.6403 & 0.3878 & 3.2123 & 310.2222 & 51.54222 & 552 & 2896 & 355 & 04 & 27.20866 & 101.187 & 1.019538 & 8.6314 & .541 \\
\hline 92048 & 70.492 & 48.91521 & 244.94 & 75392 & 86.33159 & 35.42562 & 255 & 97062 & 13.78829 & 65685 & 54 & 1868.018 & 0.904641 & 44.0917 & .795309 \\
\hline 11313 & 64.552 & 39.17932 & .6406 & 51442 & .32318 & 50.75786 & .9335 & 47199 & 12.30551 & 6.966946 & 12.68277 & 2004.555 & 1.852932 & 32.8829 & 1.780819 \\
\hline . 178327 & 3384.026 & 92.0644 & 825.3682 & 118.6058 & .92814 & 25.6938 & 28.55114 & 39.37154 & 11.50735 & 3.96264 & 2.936 & 2679.971 & 1.420439 & 487.0672 & .543 \\
\hline 074567 & 7.591 & 33.60568 & .5666 & 72.83927 & 15058 & 32.83406 & 43.30836 & 27.05174 & 9.567328 & 56126 & 11.71765 & 1536.996 & 1.204609 & 233.322 & 0.834693 \\
\hline 921255 & 02.952 & 229.9211 & 9645 & 117.1533 & 203.9299 & 30.68465 & 22 & 30.92718 & 4049 & 7.678879 & 18.69833 & 3941.899 & 1.254266 & 722.0784 & .7179 \\
\hline 84415 & 77.317 & 56.5256 & 69 & 6 & 15.555 & 747 & 8.54014 & 48.27159 & 17.41236 & 6.572117 & 849 & 23.049 & 1.118304 & 17.9614 & 1933 \\
\hline 22057 & 2.829 & 7.6602 & 18 & 897 & 18538 & 33 & 2723 & .3773 & 32 & 221 & 106 & .711 & 1.118745 & 3.7791 & 1.119098 \\
\hline 48034 & 7.223 & 87727 & 305 & 41 & & & & 58 & 02 & & 07 & & 3.87191 & 56 & \\
\hline
\end{tabular}




\begin{tabular}{|c|c|c|c|c|c|c|c|c|c|c|c|c|c|c|c|c|}
\hline Vicia lathyroides & 2.780826 & 2725.81 & 61.09457 & 200.6566 & 74.75703 & 97.40287 & 58.29411 & 50.32014 & 32.96619 & 10.73804 & 4.896973 & 10.14956 & 1570.806 & 1.687262 & 263.8694 & 1.037958 \\
\hline Vicia sativa & 5.165344 & 5085.338 & 141.1731 & 485.6874 & 91.7794 & 101.1519 & 32.35531 & 40.83888 & 41.36353 & 18.75449 & 5.37517 & 16.24421 & 3159.859 & 2.267386 & 420.445 & 1.20476 \\
\hline Vicia sativa s. nigra & 4.419814 & 4351.309 & 119.619 & 347.6643 & 118.8631 & 109.9472 & 57.24803 & 59.24003 & 42.55949 & 13.94677 & 3.923416 & 11.94024 & 2445.778 & 3.909553 & 408.8429 & 1.088176 \\
\hline Vicia sativa s. sativa & 4.354182 & 4002.699 & 107.4177 & 444.9679 & 97.19544 & 90.54854 & 30.22419 & 36.11733 & 30.37302 & 20.43933 & 4.925639 & 13.08883 & 3579.773 & 2.155407 & 389.8062 & 1.092186 \\
\hline Vicia sepium & 8.601419 & 5144.562 & 62.63693 & 705.4494 & 83.38657 & 92.12685 & 57.6603 & 54.95616 & 47.51291 & 17.99187 & 6.802707 & 27.41659 & 3258.958 & 1.325902 & 265.1474 & 1.425176 \\
\hline Vicia tetrasperma & 3.271859 & 4043.579 & 527.133 & 539.4134 & 89.57448 & 105.8365 & 28.41535 & 35.81694 & 35.76187 & 14.56783 & 4.923263 & 15.50194 & 2870.381 & 1.8111 & 366.0729 & 0.808187 \\
\hline Vicia tetrasperma s. tetrasperma & 4.215557 & 5337.32 & 92.37458 & 459.3359 & 96.99867 & 113.1416 & 34.97253 & 45.69604 & 27.48342 & 20.49639 & 5.554694 & 12.56989 & 3779.941 & 0.9561 & 311.9785 & 0.905855 \\
\hline Vinca minor & 4.163872 & 2579.227 & 34.61683 & 530.7217 & 50.31774 & 78.92207 & 36.66701 & 35.75737 & 27.92386 & 9.057712 & 6.357232 & 20.59692 & 1510.078 & 1.718178 & 134.9046 & 1.013472 \\
\hline Viola arvensis & 4.313582 & 3803.03 & 73.38229 & 518.3427 & 109.2234 & 108.6224 & 32.30143 & 43.36446 & 37.51445 & 11.09767 & 2.959109 & 13.01355 & 2376.983 & 2.572695 & 449.3208 & 1.057008 \\
\hline Viola canina & 7.061247 & 3601.358 & 43.29899 & 286.8492 & 76.81353 & 130.1873 & 81.64331 & 85.67198 & 42.54929 & 28.81655 & 6.715853 & 9.886239 & 3021.251 & 0.911748 & 354.5739 & 1.397876 \\
\hline Viola curtisii & 2.985054 & 2075.658 & 39.34967 & 205.7006 & 48.60788 & 92.31387 & 53.17841 & 46.81157 & 27.01415 & 11.59877 & 4.67323 & 12.47922 & 1515.56 & 1.634468 & 265.6663 & 1.148201 \\
\hline Viola hirta & 7.035366 & 2772.508 & 50.80909 & 439.2354 & 65.8382 & 90.96414 & 41.27895 & 40.5791 & 40.02584 & 13.37415 & 5.179249 & 29.45855 & 2001.795 & 1.546006 & 296.2768 & 1.094695 \\
\hline Viola odorata & 6.005168 & 2815.379 & 37.62174 & 1030.782 & 81.09804 & 90.83959 & 41.80974 & 35.79345 & 20.44884 & 14.49143 & 5.958017 & 22.58223 & 3351.67 & 1.312763 & 566.5543 & 1.555508 \\
\hline Viola palustris & 4.755084 & 7168.514 & 139.3743 & 469.243 & 73.61121 & 183.587 & 43.30186 & 56.02396 & 26.48784 & 23.04817 & 8.435722 & 22.94958 & 3070.07 & 1.878057 & 404.7754 & 0.725429 \\
\hline Viola persicifolia & 4.044605 & 6781.214 & 83.69042 & 406.8743 & 62.01369 & 80.17057 & 39.42469 & 31.59883 & 23.6414 & 23.4808 & 4.821764 & 8.016832 & 3749.917 & 0.647343 & 245.7769 & 0.51621 \\
\hline Viola reichenbachiana & 3.13143 & 2520.059 & 33.26443 & 332.2081 & 46.29435 & 65.80307 & 32.22057 & 25.05037 & 24.57099 & 9.540585 & 3.936189 & 15.77401 & 1473.395 & 1.125082 & 135.1429 & 0.797653 \\
\hline Viola reichenbachiana $+V$. riviniana & 4.633518 & 3148.757 & 39.66661 & 568.5735 & 57.43054 & 101.7134 & 36.43015 & 43.77839 & 39.40136 & 12.50163 & 6.437188 & 28.24537 & 2316.772 & 1.442018 & 311.5435 & 0.924324 \\
\hline Viola riviniana & 5.406789 & 2982.773 & 44.09414 & 641.481 & 61.52005 & 96.44846 & 33.60582 & 38.46456 & 36.22187 & 14.74177 & 6.240708 & 29.96151 & 2820.819 & 1.546505 & 366.5341 & 1.172173 \\
\hline Viola rupestris & 3.690679 & 2099.355 & 31.82561 & 177.549 & 59.85031 & 78.34719 & 46.53631 & 40.91301 & 24.19595 & 9.904706 & 3.682317 & 6.886225 & 1415.495 & 1.214325 & 196.667 & 0.736284 \\
\hline Viola tricolor & 3.015505 & 2896.719 & 53.38571 & 192.011 & 65.94124 & 104.9328 & 69.22599 & 65.04701 & 34.73487 & 13.22701 & 3.309639 & 14.66109 & 1857.364 & 2.420897 & 291.3152 & 1.576298 \\
\hline Vulpia bromoides & 5.415885 & 5469.521 & 62.46765 & 231.672 & 137.594 & 126.6489 & 51.57555 & 62.9067 & 32.70849 & 25.22424 & 3.632737 & 11.46455 & 2932.161 & 1.023308 & 320.1348 & 1.97517 \\
\hline Vulpia myuros & 5.523213 & 3815.832 & 50.31858 & 257.7707 & 96.72557 & 117.1147 & 48.92154 & 65.89133 & 37.97047 & 13.86501 & 2.239174 & 14.42064 & 2032.139 & 2.726647 & 311.2538 & 2.161438 \\
\hline average & 4.749155 & 5036.816 & 201.777 & 503.3916 & 98.5809 & 149.9478 & 47.36441 & 56.6664 & 39.07994 & 19.09159 & 6.369185 & 18.27921 & 3817.563 & 1.5296 & 414.3251 & 1.098787 \\
\hline s.e. & 1.992302 & 1991.766 & 201.74 & 208.1467 & 31.33951 & 64.11553 & 17.22468 & 23.31154 & 12.28371 & 7.136562 & 2.615358 & 7.430832 & 1803.76 & 0.695886 & 167.3691 & 0.4067 \\
\hline
\end{tabular}

